This document is the Accepted Manuscript version of a Published Work that appeared in final form in the Journal of Organic Chemistry, copyright ( ) American Chemical Society after peer review and technical editing by the publisher. To access the final edited and published work see https://pubs.acs.org/doi/10.1021/acs.joc.9b00050

\title{
Metal-Free Electrophilic Alkynylation of Sulfenate Anions with Ethynylbenziodoxolone Reagents
}

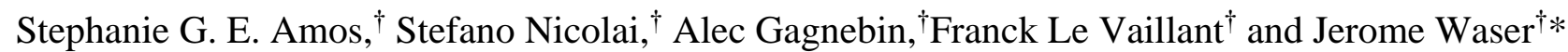

${ }^{\dagger}$ Laboratory of Catalysis and Organic Synthesis, Institute of Chemical Sciences and Engineering, Ecole

Polytechnique Fédérale de Lausanne, EPFL SB ISIC LCSO, BCH 4306, CH-1015, Lausanne, Switzer-

land.

jerome.waser@epfl.ch

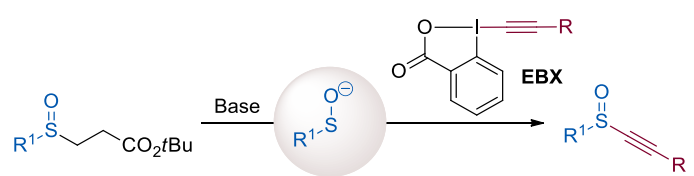

ABSTRACT: Alkynyl sulfoxides are important building blocks with unique reactivity in organic chemistry, but only few reliable methods have been reported to synthesize them. A novel route to access alkynyl sulfoxides is reported herein by using Ethynyl BenziodoXolone (EBX) reagents to trap sulfenate anions generated in-situ, via a retro-Michael reaction. The reaction takes place under metal-free and mild conditions. It is compatible with aryl, heteroaryl and alkyl sulfoxides with up to $90 \%$ yield. This practical access to alkynyl sulfoxides is expected to facilitate the application of these useful building blocks in organic synthesis.

Sulfoxides are well represented in natural products. ${ }^{1}$ They constitute also important post-translational modifications of proteins and are biological precursors of flavours and aromas. ${ }^{2}$ They have found applications in material science ${ }^{3}$ and are preponderant in pharmaceutical and bioactive compounds. Remarkable examples include the top-selling proton pump inhibitor omeprazole (1) ${ }^{4}$ the insecticide ethiprole (2), the anti-narcoleptic armodafinil (3) and the immunosuppressor oxisurane (4) (Figure 1). ${ }^{5}$ Furthermore, chiral sulfoxides are often used as ligands or auxiliaries in asymmetric synthesis. ${ }^{6}$ 


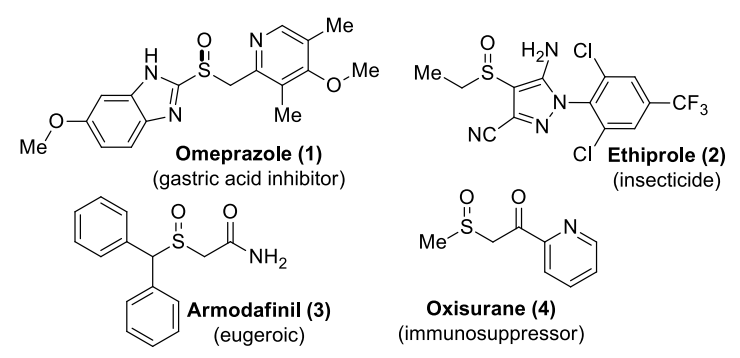

Figure 1 Examples of bioactive sulfoxide-containing compounds.

Alkynes play an essential role in numerous chemical transformations. ${ }^{7}$ More specifically, alkynyl sulfoxides have found applications as $\alpha$-sulfinyl carbene precursors (Scheme 1, A) ${ }^{8}$ They also provide easy access to allenes $(\mathbf{B})^{9}$ and they can undergo cycloadditions $(\mathbf{C}) .{ }^{10}$ They can also be converted to versatile vinyl sulfoxides via simple Michael-addition ${ }^{11}$ or reduction to both cis and trans configuration (D). ${ }^{12}$ The resulting $\alpha, \beta$-unsaturated sulfoxides are common in pharmacological compounds ${ }^{13}$ and can be used in vinylogous Pummerer rearrangements and other pericyclic reactions. ${ }^{14}$

\section{Scheme 1. Existing strategies to access alkynyl sulfoxides and their applications.}

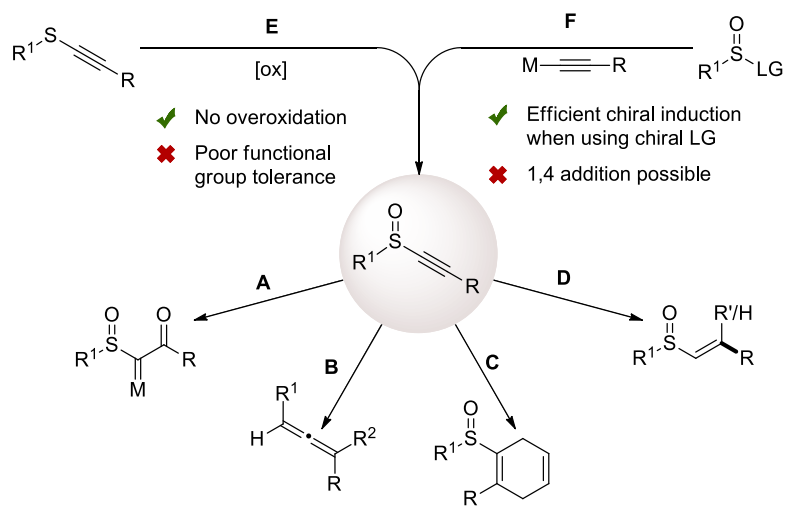

While the synthetic applications of these compounds look promising, further research and applications are limited by the lack of robust methods to access them. In fact, only two routes have been routinely used: oxidation of alkynyl sulfides and nucleophilic substitution on sulfoxide precursors (Scheme 1, $\mathbf{E}$ and F). ${ }^{11,15}$ As alkynyl sulfides are strongly deactivated, the first approach relies on harsh oxidative conditions, which are not compatible with sensitive functional groups. Concerning the second approach, the use of acetylene nucleophiles like alkynyl Grignard reagents can lead to 1,4 addition after formation of the desired compound and side-reactions with other acidic or electrophilic functional groups. ${ }^{11}$ In order 
to overcome these limitations, we envisaged the use of an inversed polarity strategy for the synthesis of alkynyl sulfoxides. In this respect, Perrio and Metzner reported in 2005 the facile synthesis of alkylated sulfoxides by trapping an in-situ generated sulfenate anion with an electrophile (Scheme $2, \mathbf{A}$ and B). ${ }^{16}$ In this method the unstable sulfenate anion ${ }^{17}$ is generated via a chemoselective retro-Michael reaction under mild conditions. This approach was later used for the synthesis of aryl and alkyl sulfoxides, in particular using palladium-catalysed cross-couplings $(\mathbf{C}) .{ }^{18}$ During completion of our work, Bolm and Chen and co-workers independently reported similar procedures for the arylation of sulfenates by using diaryliodonium salts (D). ${ }^{19}$ Nevertheless, the alkynylation of sulfenate anions has never been reported so far.

\section{Scheme 2. Retro-Michael approach for sulfenate anion generation and applications in sulfoxide} synthesis.

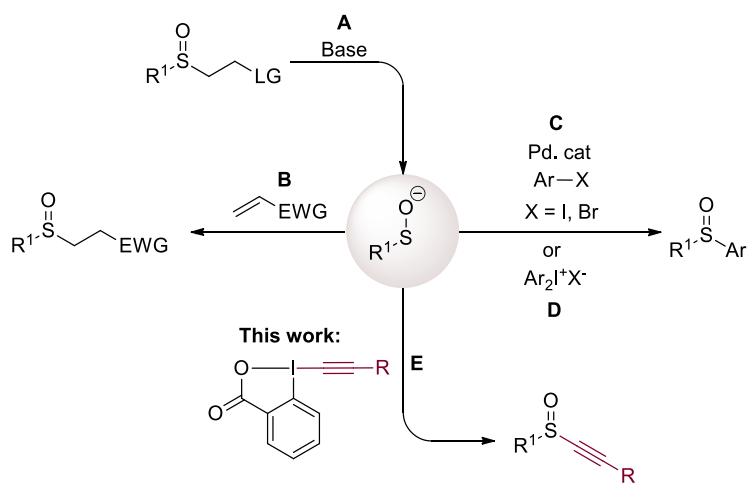

The exceptional reactivity of hypervalent iodine reagents ${ }^{20}$ has opened the door to the development of electrophilic alkynylation reactions. ${ }^{21}$ In particular, our group has shown the efficacy of cyclic Ethynyl BenziodoXolone (EBX) reagents as electrophilic alkynyl synthon transfer agents. ${ }^{22}$ Over the past decade, they have been applied for the alkynylation of various carbon and heteroatom, more generally, soft nucleophiles. $^{22}$ In particular, our group has shown the convenient use of EBX reagents for the alkynylation of thiols and sulfinates. ${ }^{23}$ These results demonstrated the high reactivity of EBX reagents towards sulfur nucleophiles. Herein, we present the first method for the synthesis of alkynyl sulfoxides by the reaction of in-situ generated sulfenates with EBX reagents (Scheme 2, E). 
We started our investigations by examining various retro-Michael sulfenate precursors under Perrio's conditions ${ }^{16}$ with TIPS-EBX $(\mathbf{9 a}$, TIPS $=$ triisopropylsilyl $)$ in THF $($ Table 1$) .{ }^{24}$

Table 1: Initial reaction condition for sulfenate precursor screening.

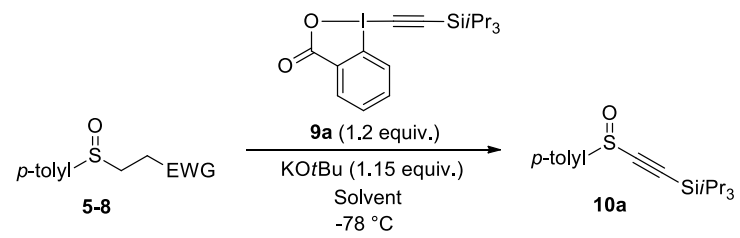

$\begin{array}{cccc}\text { entry } & \text { EWG } & \text { solvent } & \text { yield (\%) } \\ 1 & \mathrm{CO}_{2} \mathrm{Me}(\mathbf{5}) & \mathrm{THF} & 28 \\ 2 & \mathrm{CO}_{2} \mathrm{Me}(\mathbf{5}) & \mathrm{PhMe} & 48 \\ 3 & \mathrm{CO}_{2} t \mathrm{Bu}(\mathbf{6 a}) & \mathrm{PhMe} & 64 \\ 4 & \mathrm{SO}_{2} \mathrm{Ph}(\mathbf{7}) & \mathrm{PhMe} & 64 \\ 5 & \mathrm{NO}_{2}(\mathbf{8}) & \mathrm{PhMe} & 43\end{array}$

Reactions were performed with 5-8 ( $0.50 \mathrm{mmol}, 1.0$ equiv. $)$, $9 \mathrm{a}(0.60 \mathrm{mmol}, 1.2$ equiv. $)$ and $\mathrm{KO} t \mathrm{Bu}(0.59$ mmol, 1.15 equiv. $)$ in the indicated solvent $(0.16 \mathrm{M})$ at $-78{ }^{\circ} \mathrm{C}$ for $3 \mathrm{~h} .{ }^{a}$ Reaction was left overnight to achieve full conversion.

Methyl ( $p$-tolylsulfinyl)propanoate (5) ${ }^{16}$ afforded the desired alkynyl sulfoxide 10a in $28 \%$ yield (Entry 1). Changing the solvent to toluene increased the yield to $48 \%$ (Entry 2). The other precursors were then tested under the same conditions. Precursors $\mathbf{6} \mathbf{a}^{18 \mathrm{~b}}$ and $7^{18 \mathrm{c}}$ gave both better results $(64 \%$. Entries 3 and 4), whereas precursor $\mathbf{8}^{18 c}$ yielded the desired compound 10a in the same range of yield as 5 (Entry 5). More easily accessible tert-butyl ester $\mathbf{6}^{25}$ was retained as the best candidate as its use led to a more convenient protocol (shorter reaction time, easily removed volatile tert-butyl acrylate generated as a side product). 
Henceforth, we proceeded with the optimisation of the reaction conditions (Table 2). First, diluting the reaction $(0.056 \mathrm{M}$ instead of $0.16 \mathrm{M})$ and raising the temperature to $-40{ }^{\circ} \mathrm{C}$ allowed us to attain $77 \% \mathrm{NMR}$ yield (Entry 1) with a cleaner reaction profile. We then screened different bases, KOtBu proved to be the best base, even though the results with KHMDS were comparable (Entries 2-4). We tested reverse stoichiometry of $\mathbf{5}$ and $\mathbf{9 a}$, which resulted in a similar isolated yield (Entry 5). Other solvents were not more efficient than toluene (Entries 6-8). Meanwhile, tert-butyl $p$-tolylsulfinate was identified as an oxidation side product by MS and NMR analysis. Accordingly, oxygen was more carefully excluded from the reaction mixture by adding all of the dry reaction components under inert atmosphere (previously, KOtBu was added after the solvent). This modification improved the isolated yield to $81 \%$ (Entry 9). Further increasing the equivalents of KOtBu had a negative impact on the yield (Entry 10). Finally, when combining reversed stoichiometry and oxygen free conditions the desired alkynyl sulfoxide 10a was isolated in 90\% yield (Entry 11). When the reaction was performed at room temperature under the optimized conditions, a lower yield of $73 \%$ was obtained (Entry 12 ). Both tert-butyl $p$-tolylsulfinate and the alkynyl sulfone corresponding to $\mathbf{1 0 a}$ were identified as side products in this case.

Table 2: Optimisation of the reaction conditions.

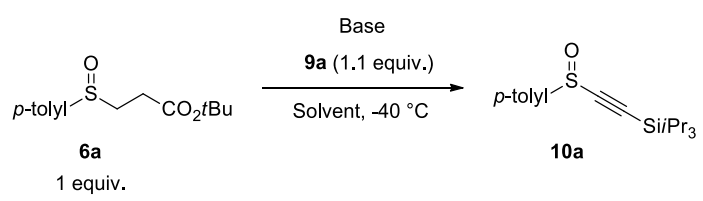

$\begin{array}{cccc}\text { entry } & \text { base } & \text { solvent } & \text { yield }(\%)^{\mathrm{a}} \\ 1 & \mathrm{KO} t \mathrm{Bu} & \mathrm{PhMe} & 70(77) \\ 2 & \mathrm{NaOt} t \mathrm{Bu} & \mathrm{PhMe} & (53) \\ 3 & \mathrm{KHDMS} & \mathrm{PhMe}\end{array}$




$\begin{array}{cccc}5^{\mathrm{c}} & \mathrm{KO} t \mathrm{Bu} & \mathrm{PhMe} & 75 \\ 6 & \mathrm{KO} t \mathrm{Bu} & \mathrm{DMF} & 28(<40) \\ 7 & \mathrm{KO} t \mathrm{Bu} & \mathrm{PhMe} / \mathrm{DCM}(9: 1) & (66) \\ 8 & \mathrm{KO} t \mathrm{Bu} & \mathrm{THF} & \\ 9^{\mathrm{d}} & \mathrm{KO} t \mathrm{Bu} & \mathrm{PhMe} & \\ 10^{\mathrm{d}} & \mathrm{KO} t \mathrm{Bu} \mathrm{e}^{\mathrm{e}} & \mathrm{PhMe} & 60 \\ 11^{\mathrm{c}, \mathrm{d}} & \mathrm{KO} t \mathrm{Bu} & \mathrm{PhMe}^{\mathrm{f}} & 90 \\ 12^{\mathrm{c}, \mathrm{d}} & \mathrm{KO} t \mathrm{Bu} & \mathrm{PhMe}^{\mathrm{g}} & 73\end{array}$

Reactions were performed with $6 \mathbf{a}(0.10 \mathrm{mmol}, 1.0$ equiv. $),(0.11 \mathrm{mmol}, 1.1$ equiv. $)$ and the indicated base $(0.105 \mathrm{mmol} 1.05$ equiv. $)$ in the indicated solvent $(0.056 \mathrm{M})$ at $-40{ }^{\circ} \mathrm{C}$ for $3 \mathrm{~h} .{ }^{\text {a Isolated yield. }}{ }^{1} \mathrm{H}-$ NMR yields using 1.0 equiv. of $\mathrm{CH}_{2} \mathrm{Br}_{2}$ as internal standard is given in parenthesis. ${ }^{\mathrm{b}} 1.1$ equiv. ${ }^{\mathrm{c}} 0.9$ equiv. of 9a. ${ }^{\mathrm{d}}$ The dry reaction components were added under inert atmosphere. ${ }^{\mathrm{e}} 1.25$ equiv. ${ }^{\mathrm{f}}$ Degassed by bubbling Ar for $40 \mathrm{~min}$ at $-40^{\circ} \mathrm{C}$ prior to addition. ${ }^{g}$ Degassing and reaction were performed at RT.

With the optimised reaction conditions in hand, we explored the scope of aryl sulfoxides (Scheme 3, A). The reaction tolerated both electron rich and electron poor para substitution affording compounds 10a-g in good to excellent yields (55-90\%). Ortho and meta substitution also provided the desired alkynyl sulfoxides $\mathbf{1 0 h}-\mathbf{j}$ in up to $81 \%$ yield. The method also allowed the synthesis of heteroaryl sulfoxides. Alkynyl pyridine sulfoxide 10k and alkynyl thiophene sulfoxide 101 were obtained in 85 and $83 \%$ yield respectively, and furanyl sulfoxide $\mathbf{1 0 m}$ in $91 \%$ yield after warming the reaction to room temperature overnight. We then moved our attention onto the synthesis of alkyl alkynyl sulfoxides (Scheme 3, B). We discovered that the reaction needed to be warmed to room temperature and left overnight to ensure optimal yields. Primary and secondary alkyl groups were both well tolerated affording 10n and 100 in 77 and $79 \%$ 
yield respectively. We then proceeded to test other EBX reagents (Scheme 3, C). TBDPS-EBX (9b) $(\mathrm{TBDPS}=$ tert-butyldiphenylsilyl $)$ and $t \mathrm{Bu}-\mathrm{EBX}(\mathbf{9 c})(t \mathrm{Bu}=t e r t$-butyl $)$ both served as efficient alkynyl transfer agents affording $\mathbf{1 0 p}$ and $\mathbf{1 0 q}$ in 81 and $48 \%$ yield respectively. However, attempts with $\mathrm{C}_{14} \mathrm{H}_{29-}$ EBX and Ph-EBX reagents were unsuccessful.

\section{Scheme 3. Scope of the alkynylation reaction.}

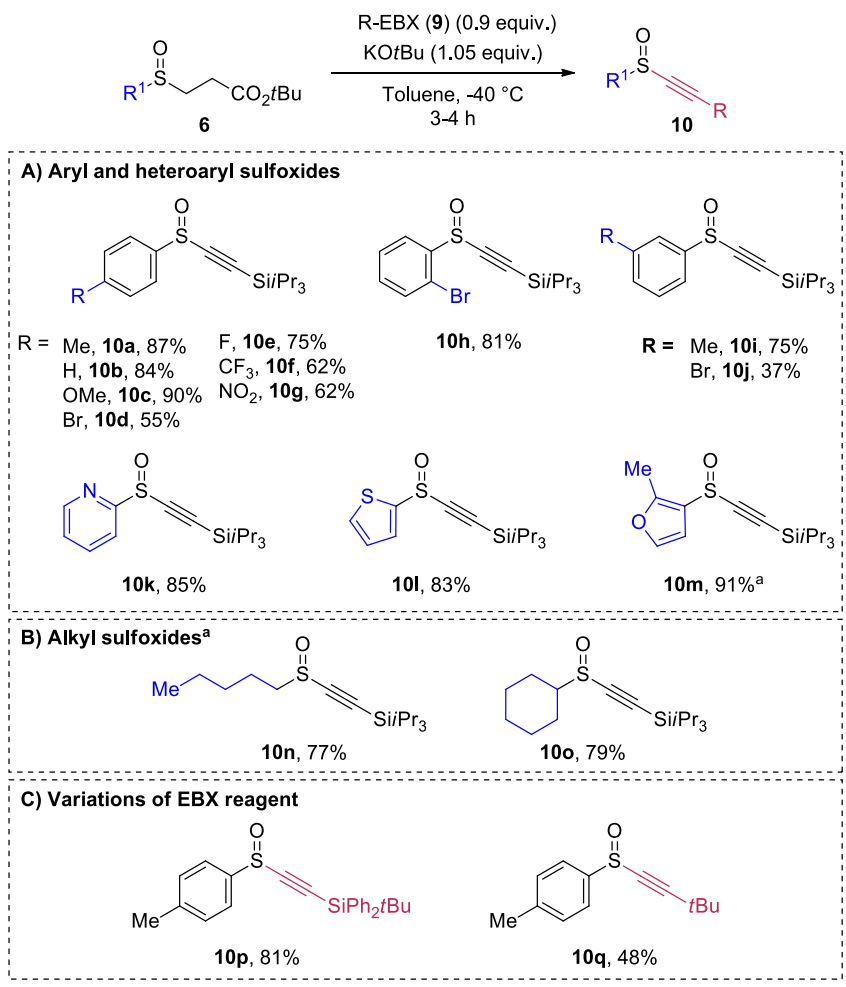

${ }^{\text {a }}$ The reaction was maintained at $-40{ }^{\circ} \mathrm{C}$ for $2 \mathrm{~h}$, then left to warm to $\mathrm{rt}$ over $18 \mathrm{~h}$.

A speculative reaction mechanism can be proposed based on literature precedence (Scheme 4). ${ }^{16,23 b, d} \mathrm{~A}$ base-initiated retro-Michael reaction first generates the unstable sulfenate anion $\mathbf{I} .{ }^{16}$ Based on our previous studies on the addition of sulfur nucleophiles onto EBX reagents, ${ }^{23 b, d}$ two mechanism pathways can then be envisaged: 1) Direct concerted addition to give sulfoxide 10 via 3-atom transition state TS1 (path a) or 2) Conjugate addition via 4-atom transition state TS2 leading to vinyl benziodoxolone II. At this stage, a 1,2-shift of either the sulfoxide (path $\mathbf{b}_{1}$ ) or the R substituent (path $\mathbf{b}_{2}$ ) together with $\alpha$-elimination of the iodine leads to sulfoxide 10. This latter step can be either concerted, or via a carbene intermediate if 
$\alpha$-elimination precedes 1,2-shift. The exact reaction pathway is expected to be dependent on the nature of the $\mathrm{R}$ group on the alkyne.

\section{Scheme 4. Speculative reaction mechanism.}

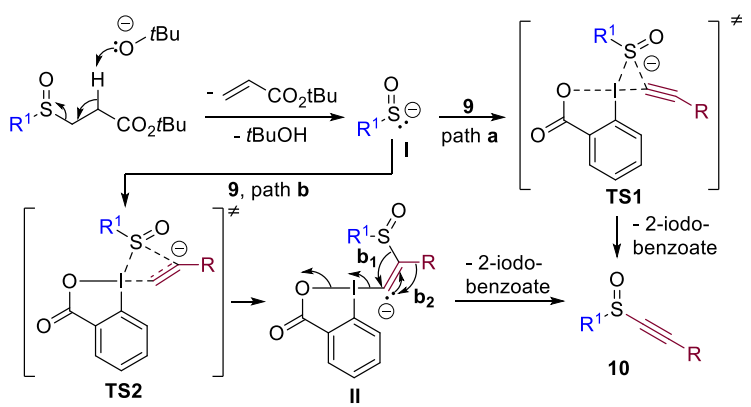

We then wondered if the functionalisation of sulfenate anions could be extended to vinylation using recently introduced Vinyl BenziodoXolone (VBX) reagents. ${ }^{26}$ To our delight, we were able to access (E)alkenyl sulfoxides $\mathbf{1 2 a}$ and $\mathbf{1 2 b}$ in yields of $52 \%$ and $25 \%$ respectively without changing the experimental procedure using the corresponding VBX reagents (eq. 1).

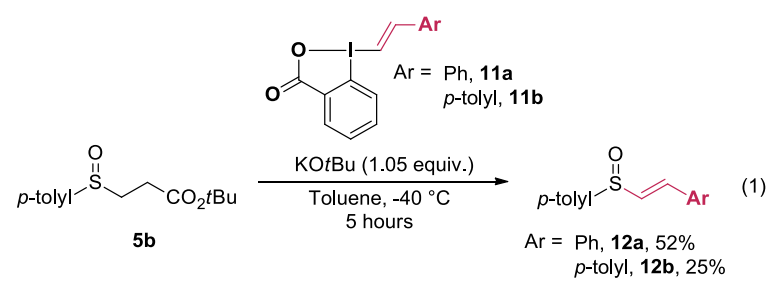

To confirm the synthetic utility of this procedure, we performed the reaction on gram-scale affording the desired alkynyl sulfoxide $\mathbf{1 0 a}$ in $86 \%$ yield (eq. 2). Finally, the free alkyne 13 was obtained in $58 \%$ after deprotection of 10a with potassium fluoride under mild conditions (eq. 3).

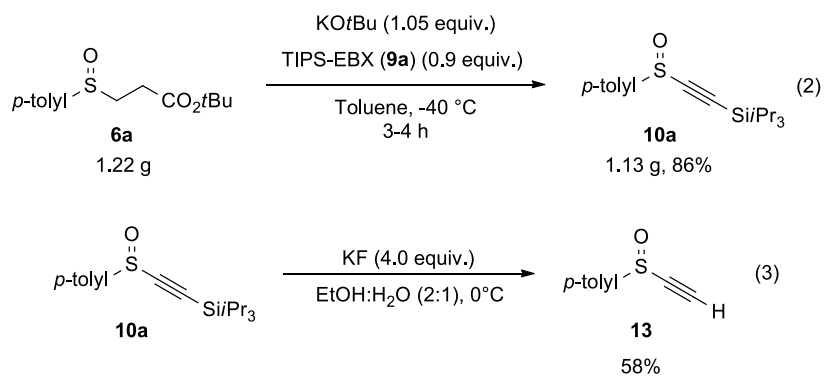

In conclusion, we have designed a new efficient route to access alkynyl sulfoxides. The use of an EBX reagent as a soft electrophilic acetylene transfer agent has allowed us to develop the first general synthetic method for alkynyl sulfoxides based on an inversed-polarity alkynylation under metal-free conditions. We 
have been able to generate in-situ alkyl, heteroaryl and aryl sulfenate anions by using potassium tertbutoxide at low temperature to initiate a retro-Michael elimination. The trapping of the resulting sulfenate anions with EBX reagents afforded alkyl and aryl alkynyl sulfoxides in high yields. Additionally, two aryl vinyl sulfoxides were also isolated when using VBX reagents. When considering the availability of the starting materials and the straightforward reaction conditions, the developed method has the potential to facilitate the use of alkynyl sulfoxides in synthetic chemistry, chemical biology and material science.

\section{Experimental Section}

\section{General methods}

HPLC grade or distilled solvents purchased from Sigma-Aldrich were used for flash chromatography. For non-air-sensitive reactions, analytical or reagent grade solvents purchased from Merck or Sigma-Aldrich were used unless specified. For all alkynylation reactions, toluene and THF dried by passage over activated alumina under nitrogen atmosphere (H2O content $<7$ ppm, Karl-Fisher titration) or anhydrous solvents purchased from Sigma-Aldrich were used. Toluene was degassed by bubbling with a balloon of argon when mentioned. Reagents were purchased from Sigma-Aldrich, Acros, TCI, Fluorochem, Fluka, VWR or Merck, unless specified. Chromatographic purification was performed as flash chromatography using Macherey-Nagel silica 40-63, $60 \AA$ A, using the solvents indicated as eluent with 0.1-0.5 bar pressure or using Biotage Isolera Spektra One with pre-packaged silica cartridges purchased from Buchi, models: Sepacore or GraceResolve (4 g, 12 g, 25 g). TLC was performed on Merck silica gel 60 F254 TLC glass plates or aluminium plates and visualized with UV light, permanganate stain, CAN stain. Melting points were measured using a calibrated Buchi B-540 apparatus using open glass capillaries. 1H-NMR spectra were recorded on a Brucker DPX-400, $400 \mathrm{MHz}$, in $\mathrm{CDCl}_{3},\left(\mathrm{CD}_{3}\right)_{2} \mathrm{SO}$ or $\mathrm{CD}_{3} \mathrm{OD}$. All signals are reported in ppm using the residual solvent signal as internal reference $\left(\mathrm{CDCl}_{3}: 7.26 \mathrm{ppm},\left(\mathrm{CD}_{3}\right)_{2} \mathrm{SO}: 2.50 \mathrm{ppm}\right.$ or

$\mathrm{CD}_{3} \mathrm{OD}: 3.31 \mathrm{ppm}$ ). The data is reported as (multiplicity, coupling constants in $\mathrm{Hz}$, integration, interpretation) using these abbreviations: $\mathrm{s}=$ singulet, $\mathrm{d}=$ doublet, $\mathrm{t}=$ triplet, $\mathrm{m}=$ multiplet, $\mathrm{bs}=$ broad signal. 
13C $\{1 \mathrm{H}\}-\mathrm{NMR}$ spectra were carried out with $1 \mathrm{H}$ decoupling on a Brucker DPX-400, $101 \mathrm{MHz}$. All signals are reported in ppm using the residual solvent signal as internal reference $\left(\mathrm{CDCl}_{3}: 77.0 \mathrm{ppm}\right.$, $\left(\mathrm{CD}_{3}\right)_{2} \mathrm{SO}: 39.5$ ppm or $\left.\mathrm{CD}_{3} \mathrm{OD}: 49.0\right)$. Infrared spectra were obtained on a JASCO FT-IR B4100 spectrophotometer with an ATR PRO410-S and a ZnSe prisma and are reported in $\mathrm{cm}^{-1}$ as $(\mathrm{w}=$ weak, $\mathrm{m}=$ medium, $\mathrm{s}=$ strong). High resolution mass spectrometric measurements were performed by the mass spectrometry service of ISIC at the EPFL on a MICROMASS (ESI) Q-TOF Ultima API. Cooling baths were assured by using Huber TC100E and TC50E cryostats with an ethyl acetate bath.

\section{Synthesis of 1-Hydroxy-1 $\lambda 3,2$-benziodoxol-3(1H)-one (14)}

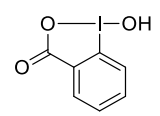

Following a reported procedure, ${ }^{27} \mathrm{NaIO}_{4}(7.24 \mathrm{~g}, 33.8 \mathrm{mmol}, 1.05$ equiv.) and 2-iodobenzoic acid (8.00 g, $32.2 \mathrm{mmol}, 1.00$ equiv.) were suspended in $30 \%$ (v:v) aq. AcOH (48 mL). The mixture was stirred vigorously and refluxed for 4 hours. The reaction mixture was then diluted with cold water $(180 \mathrm{~mL})$ and allowed to cool to $\mathrm{rt}$, protecting it from light. After 1 hour, the crude product was collected by filtration, washed on the filter with ice water $(3 \times 20 \mathrm{~mL})$ and acetone $(3 \times 20 \mathrm{~mL})$, then airdried in the dark to give the pure product $\mathbf{1 4}(8.3 \mathrm{~g}, 31 \mathrm{mmol}, 98 \%)$ as a colorless solid. ${ }^{\mathbf{1}} \mathbf{H}-\mathbf{N M R}(400$ $\left.\mathrm{MHz},\left(\mathrm{CD}_{3}\right)_{2} \mathrm{SO}\right) \delta=8.02(\mathrm{dd}, J=7.7,1.4 \mathrm{~Hz}, 1 \mathrm{H}), 7.97(\mathrm{~m}, 1 \mathrm{H}), 7.85(\mathrm{dd}, J=8.2,0.7 \mathrm{~Hz}, 1 \mathrm{H}), 7.71(\mathrm{td}$, $J=7.6,1.2 \mathrm{~Hz}, 1 \mathrm{H}) \mathrm{ppm} .{ }^{13} \mathbf{C}\{\mathbf{1 H}\}-\mathbf{N M R}\left(101 \mathrm{MHz},\left(\mathrm{CD}_{3}\right)_{2} \mathrm{SO}\right) \delta=167.7,134.5,131.5,131.1,130.4$, 126.3, $120.4 \mathrm{ppm}$. The values of the NMR spectra are in accordance with reported literature data. ${ }^{27}$

\section{Synthesis of triisopropylsilyl(trimethylsilyl)acetylene (15)}

TIPs $=$ TMs $\quad$ Following a reported procedure ${ }^{28} n$-butyl lithium $(2.5 \mathrm{M}$ in hexanes, $12.0 \mathrm{~mL}, 29.9 \mathrm{mmol}$, 0.98 equiv.) was added dropwise to a solution of ethynyltrimethylsilane ( $3.0 \mathrm{~g}, 30 \mathrm{mmol}, 1.0$ equiv.) in THF (48 mL) at $-78{ }^{\circ} \mathrm{C}$ in a two-neck $100 \mathrm{~mL}$ oven-dried flask under nitrogen. The mixture was warmed to $0{ }^{\circ} \mathrm{C}$ and stirred for 5 minutes. The mixture was cooled back to $-78^{\circ} \mathrm{C}$ and chlorotriisopropylsilane $(6.4$ $\mathrm{mL}, 30 \mathrm{mmol}, 1$ equiv.) was added dropwise. The mixture was then warmed to room temperature and stirred overnight. The reaction was then quenched with aq. sat. ammonium chloride $(40 \mathrm{~mL})$. The reaction mixture was extracted with diethyl ether $(3 \times 60 \mathrm{~mL})$. The organic phases were combined, washed with 
water and brine, dried with $\mathrm{MgSO}_{4}$, filtered and concentrated under reduced pressure to obtain a colourless liquid which was further purified by Kugelrohr distillation $\left(56-57^{\circ} \mathrm{C}, 0.25 \mathrm{mmHg}\right)$ affording 15 as a clear oil (4.57 g, 18 mmol, 85\% yield). ${ }^{1} \mathbf{H}-\mathbf{N M R}\left(400 \mathrm{MHz}, \mathrm{CDCl}_{3}\right) \delta=1.08(\mathrm{~m}, 21 \mathrm{H}), 0.18$ (s, 9H) ppm. The values of the ${ }^{1} \mathrm{H}-\mathrm{NMR}$ spectra are in accordance with reported literature data. ${ }^{28}$

\section{Synthesis of 1-[(Triisopropylsilyl)ethynyl]-1ג3,2-benziodoxol-3(1H)-one (TIPS-EBX, 9a)}

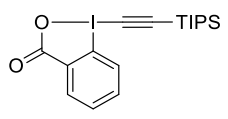

Following a reported procedure, ${ }^{29}$ 2-iodosylbenzoic acid (14) (21.7 g, 82.0 mmol, 1.0 equiv.) was introduced into an oven-dried three-neck $1 \mathrm{~L}$ flask equipped with a magnetic stirrer. After 3 vacuum/nitrogen cycles, anhydrous acetonitrile $(500 \mathrm{~mL})$ was added via cannula and cooled to $0{ }^{\circ} \mathrm{C}$. Trimethylsilyltriflate $(16.4 \mathrm{~mL}, 90.0 \mathrm{mmol}, 1.1$ equiv.) was added dropwise via a dropping funnel over 30 minutes (no temperature increase was observed). After 15 minutes, triisopropylsilyl(trimethylsilyl)acetylene (15) (23.0 g, 90.0 mmol, 1.1 equiv.) was added via cannula over 15 min (no temperature increase was observed). After $30 \mathrm{~min}$, the suspension became an orange solution. After 10 minutes, pyridine $(7.0 \mathrm{~mL}, 90 \mathrm{mmol}, 1.1$ equiv.) was added via syringe. After $15 \mathrm{~min}$, the reaction mixture was transferred to a one-neck $1 \mathrm{~L}$ flask and concentrated under vacuum to provide an yellowish solid. The latter was dissolved in DCM $(200 \mathrm{~mL})$ and transferred to a $1 \mathrm{~L}$ separatory funnel. The organic layer was added and washed with aq. $\mathrm{HCl}(1.0 \mathrm{M} ; 200 \mathrm{~mL})$ and the aqueous layer was extracted with dichloromethane $(200 \mathrm{~mL})$. The organic layers were combined, washed with a saturated aqueous solution of $\mathrm{Na}-$ $\mathrm{HCO}_{3}\left(2\right.$ x $200 \mathrm{~mL}$ ), dried over $\mathrm{MgSO}_{4}$, filtered and concentrated under reduced pressure. Recrystallization from acetonitrile (ca. $120 \mathrm{~mL}$ ) afforded $9 \mathbf{a}(30.1 \mathrm{~g}, 70.2 \mathrm{mmol}, 86 \%)$ as a colorless crystalline solid. mp: $170-176{ }^{\circ} \mathrm{C}$ (decomposition); ${ }^{1} \mathbf{H}-\mathbf{N M R}\left(400 \mathrm{MHz}, \mathrm{CDCl}_{3}\right) \delta=8.44(\mathrm{~m}, 1 \mathrm{H}), 8.29(\mathrm{~m}, 1 \mathrm{H}), 7.77(\mathrm{~m}$, 2H), $1.16(\mathrm{~m}, 21 \mathrm{H}) \mathrm{ppm} .{ }^{\mathbf{1 3}} \mathbf{C}\{\mathbf{1 H}\}-\mathbf{N M R}\left(101 \mathrm{MHz}, \mathrm{CDCl}_{3}\right) \delta=166.4,134.6,132.3,131.4,131.4,126.1$, 115.6, 114.1, 64.6, 18.4, $11.1 \mathrm{ppm}$. The values of the NMR spectra are in accordance with reported literature data. $^{29}$

\section{Synthesis of tert-butyldiphenylsilyl(trimethylsilyl)acetylene (16)}


${ }_{\mathrm{TBDPS}}=$ TMs Following a reported procedure, ${ }^{30} n$-butyllithium $(2.5 \mathrm{M}$ in hexanes, $8.0 \mathrm{~mL}, 20 \mathrm{mmol}, 0.98$ equiv) was added dropwise to a stirred solution of ethynyltrimethylsilane (2.90 mL, $20.4 \mathrm{mmol}, 1.0$ equiv) in THF $(30 \mathrm{~mL})$ at $-78^{\circ} \mathrm{C}$. The mixture was then warmed to $0{ }^{\circ} \mathrm{C}$ and stirred for 5 minutes. The mixture was then cooled back to $-78^{\circ} \mathrm{C}$ and tertbutylchlorodiphenylsilane $(6.4 \mathrm{~mL}, 30 \mathrm{mmol}, 1.0$ equiv.) was added dropwise. The mixture was then allowed to warm to room temperature and stirred overnight. A saturated aqueous solution of ammonium chloride $(30 \mathrm{~mL})$ was added, and the reaction mixture was extracted with diethyl ether $(2 \times 50 \mathrm{~mL})$. The organic layer was washed with water and brine, then dried over $\mathrm{MgSO}_{4}$, filtered and concentrated under reduced pressure to obtain a colorless liquid which was further purified by Kugelrohr distillation $\left(150{ }^{\circ} \mathrm{C}, 0.25 \mathrm{mmHg}\right)$ to yield $\mathbf{1 6}(2.95 \mathrm{~g}, 8.76 \mathrm{mmol}, 44 \%$ yield $)$ as a colorless liquid. ${ }^{1} \mathrm{H}-\mathrm{NMR}\left(400 \mathrm{MHz}_{\mathrm{CDCl}}\right) \delta=7.80(\mathrm{~m}, 4 \mathrm{H}), 7.38(\mathrm{~m}, 6 \mathrm{H}), 1.08(\mathrm{~s}, 9 \mathrm{H}), 0.27(\mathrm{~s}$, 9H) ppm. ${ }^{13} \mathbf{C}\{\mathbf{1 H}\}-\mathbf{N M R}\left(101 \mathrm{MHz}, \mathrm{CDCl}_{3}\right) \delta=135.6,133.2,129.5,127.7,119.0,108.7,27.0,18.5,-$ $0.0 \mathrm{ppm}$. The values of the NMR spectra are in accordance with reported literature data. ${ }^{30}$

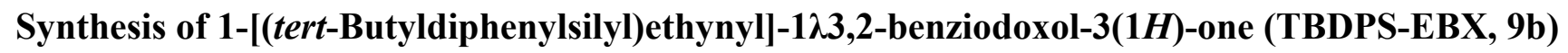

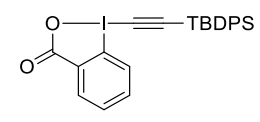

Following a reported procedure, ${ }^{31}$ trimethylsilyltriflate (freshly distilled; $1.6 \mathrm{~mL}, 8.7$

mmol, 1.1 equiv.) was added dropwise to a stirred solution of 2-iodosylbenzoic acid (14) (2.07 g, $7.90 \mathrm{mmol}, 1.0$ equiv.) in acetonitrile $(30 \mathrm{~mL})$. tert-Butyldiphenyl((trimethylsilyl)ethynyl)silane (16) (2.95 g, $3.70 \mathrm{mmol}, 1.1$ equiv.) was then added dropwise, followed, after $15 \mathrm{~min}$, by the addition of pyridine $(710 \mu \mathrm{L}, 3.70 \mathrm{mmol}, 1.1$ equiv.). The mixture was stirred 10 minutes. The solvent was then removed under reduced pressure and the resulting yellow crude oil was dissolved in dichloromethane. The organic layer was washed with aq. $\mathrm{HCl}(1.0 \mathrm{M}, 15 \mathrm{~mL})$, and the aqueous layer was extracted with dichloromethane. The organic layers were combined, washed with a sat. aq. $\mathrm{NaHCO}_{3}$, dried over $\mathrm{MgSO}_{4}$, filtered and concentrated under reduced pressure. The resulting oil was stirred in hexane and ether and then concentrated under vacuum to afford a colorless solid. Recrystallization from acetonitrile (ca $20 \mathrm{~mL}$ ) afforded 9b (2.77 g, $5.42 \mathrm{mmol}, 69 \%$ yield) as a colorless solid. ${ }^{1} \mathbf{H}-\mathbf{N M R}\left(400 \mathrm{MHz}, \mathrm{CDCl}_{3}\right) \delta=8.43$ $(\mathrm{d}, J=6.5 \mathrm{~Hz}, 1 \mathrm{H}), 8.29(\mathrm{~d}, J=8.2 \mathrm{~Hz}, 1 \mathrm{H}), 7.82(\mathrm{~d}, J=6.6 \mathrm{~Hz}, 4 \mathrm{H}), 7.75(\mathrm{t}, J=7.2 \mathrm{~Hz}, 1 \mathrm{H}), 7.66(\mathrm{~m}$, 
1H), 7.53-7.41 (m, 6H), $1.21(\mathrm{~s}, 9 \mathrm{H}) \mathrm{ppm} .{ }^{13} \mathbf{C}\{\mathbf{1 H}\}-\mathbf{N M R}\left(101 \mathrm{MHz}, \mathrm{CDCl}_{3}\right) \delta=166.6,135.5,134.8$, $132.4,131.5,131.3,130.2,128.1,126.3,116.0,112.2,68.5,27.0,18.7 \mathrm{ppm}$. The signal corresponding to one aromatic carbon was not resolved. The values of the NMR spectra are in accordance with reported literature data. ${ }^{31}$

\section{Synthesis of 1-[(tert-Butylsilyl)ethynyl]-123,2-benziodoxol-3(1H)-one (tBu-EBX, 9c)}

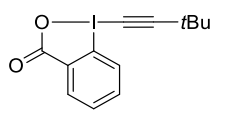

Following a reported procedure, ${ }^{31}$ trimethylsilyltriflate (freshly distilled; $1.5 \mathrm{~mL}, 8.4$ mmol, 1.0 equiv) was added dropwise to a stirred solution of 2-iodosylbenzoic acid (14) (2.69 g, $10.1 \mathrm{mmol}, 1.2$ equiv) in acetonitrile (30 mL). Commercially available 3,3-dimethylbut-1-yn-1yl)trimethylsilane (1.30 g, $8.42 \mathrm{mmol}, 1.0$ equiv) was then added dropwise, followed, after $15 \mathrm{~min}$, by the addition of pyridine $(680 \mu \mathrm{L}, 8.42 \mathrm{mmol}, 1.0$ equiv). The mixture was stirred $10 \mathrm{~min}$. The solvent was then removed under reduced pressure. Dichloromethane and aq. $\mathrm{NaOH}(1.0 \mathrm{M})$ were added. The resulting suspension was filtered. The layers were separated and the aqueous layer was extracted with dichloromethane. The organic layers were combined, dried over $\mathrm{MgSO}_{4}$, filtered and the solvent was evaporated under reduced pressure. Two recrystallisations (with hot filtration) from acetonitrile were necessary to afford 1-[3,3- dimethylbutynyl]-1,2-benziodoxol-3(1H)-one (9c) (1.43g, $4.36 \mathrm{mmol}, 57 \%$ yield) as a colorless solid. ${ }^{1} \mathrm{H}-\mathbf{N M R}\left(400 \mathrm{MHz}, \mathrm{CDCl}_{3}\right) \delta=8.39(\mathrm{~m}, 1 \mathrm{H}), 8.12(\mathrm{~m}, 1 \mathrm{H}), 7.75(\mathrm{~m}, 2 \mathrm{H}), 1.37$ (s, $\left.9 \mathrm{H}\right) \mathrm{ppm}$. The values of the NMR spectra are in accordance with reported literature data. ${ }^{31}$

\section{General procedure A: Synthesis of VBX reagents (11a and 11b):}

Following a slightly modified reported proceedure, ${ }^{32}$ to a suspension of 2-iodosylbenzoic acid (14) (0.343 g, $1.30 \mathrm{mmol}, 1.00$ equiv. $)$ in dry dichloromethane $(13 \mathrm{~mL})$ was added TMSOTf $(0.27 \mathrm{~mL}, 1.5 \mathrm{mmol}$, 1.15 equiv.) dropwise over 10 minutes and stirred for 30 minutes at room temperature. Afterwards, the corresponding vinyl boronic acid (20a or $\mathbf{2 0 b})$ (1.50 mmol, 1.15 equiv.) was added and the reaction mixture was stirred for 2 hours at room temperature. Pyridine $(0.12 \mathrm{~mL}, 1.5 \mathrm{mmol}, 1.15$ equiv. $)$ was added, after which the mixture was stirred for additional 10 minutes at room temperature. The solvent was then removed under reduced pressure. The resulting solid was dissolved in dihloromethane $(20 \mathrm{~mL})$ and 
washed with aqueous $\mathrm{HCl}(1.0 \mathrm{M} ; 10 \mathrm{~mL})$. The aqueous layer was extracted with dichloromethane $(3 \mathrm{x}$ $20 \mathrm{~mL}$ ). The organic layers were combined, washed successively with a saturated aqueous solution of $\mathrm{NaHCO}_{3}(40 \mathrm{~mL})$ and water $(3 \times 20 \mathrm{~mL})$, dried over $\mathrm{MgSO}_{4}$, filtered, and concentrated under reduced pressure. The resulting solid was dissolved again in dichloromethane (minimum amount until dissolution) and precipitated in diethyl ether (ca. $100 \mathrm{~mL}$ ). After precipitation at $4{ }^{\circ} \mathrm{C}$ for 2 hours, the solid was collected by filtration and washed with $\mathrm{Et}_{2} \mathrm{O}$ to afford the corresponding VBX reagent.

\section{(E)-1-Styryl-1 $\lambda^{3}$-benzo[d][1,2]iodaoxol-3(1H)-one (11a)}

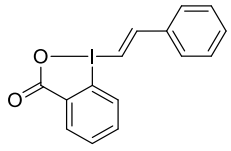

Following the general procedure A, 11a was obtained as a colourless solid from trans2-phenylvinylboronic acid (0.221 g, $1.50 \mathrm{mmol})$ and 2-iodosylbenzoic acid (14) (0.343 g, $1.30 \mathrm{mmol})$. Yield: $77 \%$ (0.351 g, $1.00 \mathrm{mmol}) .{ }^{1} \mathbf{H}-\mathbf{N M R}\left(400 \mathrm{MHz}, \mathrm{CD}_{3} \mathrm{OD}\right) \delta=8.32-8.25(\mathrm{~m}, 1 \mathrm{H})$, $7.97(\mathrm{~d}, J=15.5 \mathrm{~Hz}, 1 \mathrm{H}), 7.77-7.63(\mathrm{~m}, 6 \mathrm{H}), 7.54-7.45(\mathrm{~m}, 3 \mathrm{H}) \mathrm{ppm} .{ }^{\mathbf{1 3}} \mathbf{C}\{\mathbf{1 H}\}-\mathbf{N M R}(101 \mathrm{MHz}$ $\left.\mathrm{CD}_{3} \mathrm{OD}\right) \delta=170.1,155.8,136.7,135.3,134.5,133.3,132.1,131.8,130.2,129.0,129.0,115.5,100.0$ ppm. The values of the NMR spectra are in accordance with reported literature data. ${ }^{32}$

\section{(E)-1-(4-Methylstyryl)-1 $\lambda^{3}$-benzo $[d][1,2]$ iodaoxol-3(1H)-one (11b)}

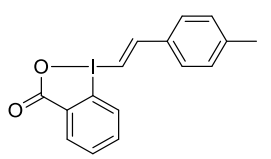

Following the general procedure $\mathbf{A}, \mathbf{1 1 b}$ was obtained as a colourless solid from trans2-(4-methylphenyl)vinylboronic acid (0.242 g, $1.50 \mathrm{mmol})$ and 2-iodosylbenzoic acid (14) $(0.343 \mathrm{~g}, 1.30 \mathrm{mmol})$. Yield: $71 \%$ (0.335 g, $0.920 \mathrm{mmol}) .{ }^{1} \mathbf{H}-\mathbf{N M R}\left(400 \mathrm{MHz}, \mathrm{CD}_{3} \mathrm{OD}\right) \delta=8.32-$ $8.25(\mathrm{~m}, 1 \mathrm{H}), 7.92(\mathrm{~d}, J=15.4 \mathrm{~Hz}, 1 \mathrm{H}), 7.76-7.64(\mathrm{~m}, 3 \mathrm{H}), 7.62-7.54(\mathrm{~m}, 3 \mathrm{H}), 7.31(\mathrm{~d}, J=7.9 \mathrm{~Hz}$ 2H), $2.42(\mathrm{~s}, 3 \mathrm{H})$ ppm. ${ }^{\mathbf{1 3}} \mathbf{C}\{\mathbf{1 H}\}$-NMR (101 MHz, CD $\left.3 \mathrm{OD}\right) \delta=169.9,155.7,142.8,135.0,134.3,133.8$ $133.1,131.6,130.6,128.8,115.3,98.1,21.3 \mathrm{ppm}$. The values of the NMR spectra are in accordance with reported literature data. ${ }^{32}$

\section{1-Chloro-1,3-dihydro-3,3-bis(trifluoromethyl)-1,2-benziodoxole (17)} Following a reported procedure, ${ }^{33} \mathrm{TMEDA}$ (distilled over $\left.\mathrm{KOH}\right)(0.63 \mathrm{~mL}, 4.1 \mathrm{mmol}, 0.2$
equiv) was added to a solution of $n \operatorname{BuLi}(2.5 \mathrm{M}$ in hexanes, $18.3 \mathrm{~mL}, 45.8 \mathrm{mmol}, 2.2$ equiv). 
After $15 \mathrm{~min}$, the cloudy solution was cooled to $0{ }^{\circ} \mathrm{C}$ and $\alpha, \alpha$-bis(trifluoromethyl)benzyl alcohol (3.5 $\mathrm{mL}$, $21 \mathrm{mmol}, 1$ equiv) in THF ( $3 \mathrm{~mL})$ was added dropwise. The reaction was stirred $30 \mathrm{~min}$ at $0{ }^{\circ} \mathrm{C}$ and then $18 \mathrm{~h}$ at rt. $\mathrm{I}_{2}\left(5.6 \mathrm{~g}, 22 \mathrm{mmol}, 1.06\right.$ equiv.) was then added portion-wise at $0{ }^{\circ} \mathrm{C}$ and the mixture stirred at $0{ }^{\circ} \mathrm{C}$ for $30 \mathrm{~min}$ and $4 \mathrm{~h}$ at $\mathrm{rt}$. The reaction was quenched with a saturated aqueous solution of $\mathrm{NH}_{4} \mathrm{Cl}$. Diethyl ether $(50 \mathrm{~mL})$ was added and the layers were separated. The aqueous layer was then extracted twice with diethyl ether $(2 \times 50 \mathrm{~mL})$. The organic layers were combined, washed twice with a saturated aqueous solution of $\mathrm{NaS}_{2} \mathrm{O}_{3}(2 \times 50 \mathrm{~mL})$, dried over $\mathrm{MgSO}_{4}$, filtered and reduced to afford $7.83 \mathrm{~g}$ of 2iodo- $\alpha, \alpha$-bis(trifluoromethyl)-benzenemethanol as an orange oil which was used without further purification. The crude oil was dissolved in dichloromethane $(20 \mathrm{~mL})$ in the dark under air. $t \mathrm{BuOCl}(2.6 \mathrm{~mL}$, $22 \mathrm{mmol}, 1.05$ equiv.) was then added dropwise at $0{ }^{\circ} \mathrm{C}$. After $30 \mathrm{~min}$, the resulting suspension was filtered to afford $17(3.52 \mathrm{~g}, 8.70 \mathrm{mmol}, 42 \%)$ as a yellow oil. The mother liquors were carefully reduced to one third and filtered to afford $17(2.33 \mathrm{~g}, 5.76 \mathrm{mmol}, 28 \%)$ as a yellow solid. Combined yield: $70 \%$. ${ }^{\mathbf{1} H-N M R}\left(400 \mathrm{MHz}, \mathrm{CDCl}_{3}\right) \delta=8.09(\mathrm{~d}, 1 \mathrm{H}, J=8.4 \mathrm{~Hz}), 7.85(\mathrm{~m}, 1 \mathrm{H}), 7.73(\mathrm{~m}, 2 \mathrm{H}) \mathrm{ppm} .{ }^{\mathbf{1 3}} \mathbf{C}\{\mathbf{1 H}\}-$ NMR $\left(101 \mathrm{MHz} \mathrm{CDCl}_{3}\right) \delta=133.8,132.1,131.6,129.7,128.5,122.8$ (q, $\left.289 \mathrm{~Hz}\right), 113.4,84.8$ ppm. The values of the NMR spectra are in accordance with reported literature data. ${ }^{34}$

\section{1-Hydroxy-3,3-bis(trifluoromethyl)-3-(1H)-1,2-benziodoxole (18)}

Following a reported procedure, ${ }^{35} \mathrm{Et}_{3} \mathrm{BnNCl}(0.083 \mathrm{~g}, 0.36 \mathrm{mmol}, 0.05$ equiv) was added to a stirring solution of 17 (3.00 g, $7.41 \mathrm{mmol}, 1$ equiv) in dichloromethane $(50 \mathrm{~mL})$ and $\mathrm{KOH}(0.415 \mathrm{~g}$, $7.41 \mathrm{mmol}, 1$ equiv) in water $(8 \mathrm{~mL})$. The reaction was stirred for 3.5 hours under air. The organic layer was separated and dried over $\mathrm{MgSO}_{4}$. The resulting solid was purified over a silica plug with ethyl acetate, then recrystallised in ethyl acetate and washed with pentane to afford $\mathbf{1 8}(1.24 \mathrm{~g}, 3.21 \mathrm{mmol}, 43 \%)$ as a colourless solid. The mother liquors were reduced and recrystallised in ethyl acetate to afford a second batch of $18(0.279 \mathrm{~g}, 0.723 \mathrm{mmol}, 10 \%)$ as a colourless solid. Combined yield: $53 \%$. ${ }^{1} \mathbf{H}-\mathbf{N M R}(400$ $\left.\mathrm{MHz},\left(\mathrm{CD}_{3}\right)_{2} \mathrm{SO}\right) \delta=7.96(\mathrm{~m}, 2 \mathrm{H}), 7.73(\mathrm{~m}, 2 \mathrm{H}) \mathrm{ppm} .{ }^{13} \mathbf{C}\{\mathbf{1 H}\}-\mathbf{N M R}\left(101 \mathrm{MHz},\left(\mathrm{CD}_{3}\right)_{2} \mathrm{SO}\right) \delta=133.3$, 
131.0, 130.8, 128.9, 127.9, $123.4(\mathrm{q}, J=290 \mathrm{~Hz}), 117.2,83.7(\mathrm{~m}) \mathrm{ppm}$. The values of the NMR spectra are in accordance with literature data. ${ }^{35}$

\section{1-[(Triisopropylsilyl)ethynyl]-3,3-bis(trifluoromethyl)-3(1H)-1,2-benziodoxole (19)}

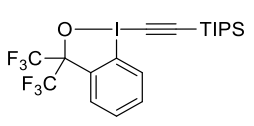

TMSOTf (310 $\mu \mathrm{L}, 1.71 \mathrm{mmol}, 1.1$ equiv) was added to 18 (0.600 g, $1.55 \mathrm{mmol}, 1.0$ equiv) in dichloromethane $(20 \mathrm{~mL})$ at $\mathrm{rt}$. After $20 \mathrm{~min}$, the solution was reduced and the resulting oil was dissolved in acetonitrile $(30 \mathrm{~mL})$. (trimethylsilyl)(triisopropylsilyl)acetylene (16) $(0.514$ g, $1.71 \mathrm{mmol}, 1.1$ equiv) was added and after 20 min pyridine (76 $\mu \mathrm{L}, 0.94 \mathrm{mmol}, 0.6$ equiv) was added. The reaction was then reduced under vacuum, dissolved in diethyl ether and filtered over a silica plug (eluant: diethyl ether). The resulting solid was purified by column chromatography (Petroleum ether:diethyl ether 95:5) to afford $19(0.816 \mathrm{~g}, 1.48 \mathrm{mmol}, 95 \%)$ as a colourless solid. ${ }^{1} \mathbf{H}-\mathbf{N M R}(400 \mathrm{MHz}$, Choroform- $d) \delta=8.36(\mathrm{dd}, 1 \mathrm{H}, J=7.9,1.7 \mathrm{~Hz}), 7.84(\mathrm{~d}, 1 \mathrm{H}, J=6.7 \mathrm{~Hz}), 7.68(\mathrm{~m}, 2 \mathrm{H}), 1.15(\mathrm{~m}, 21 \mathrm{H})$ ppm. ${ }^{13} \mathbf{C}\left\{{ }^{1} \mathbf{H}\right\}$-NMR (101 MHz, $\left.\mathrm{CDCl}_{3}\right) \delta=132.7,131.1,129.9,129.9(\mathrm{~m}), 128.2,123.6$ (q, $\left.288 \mathrm{~Hz}\right)$, $112.1,110.8,81.4(\mathrm{~m}), 69.7,18.5,11.2 \mathrm{ppm}$.

\section{Phenyl(triisopropylsilyl)iodonium triflate (20)}

$\stackrel{\ominus}{\circ} \quad$ Following a reported procedure, ${ }^{36}$ phenyliodonium diacetate $(2.53 \mathrm{~g}, 7.85 \mathrm{mmol}, 1.00$ equiv) was diluted with dichloromethane $(7 \mathrm{~mL})$ and the mixture was stirred for 5 minutes. Triflic anhydride $(0.60 \mathrm{~mL}, 3.9 \mathrm{mmol}, 0.50$ equiv. $)$ was added dropwise at $0{ }^{\circ} \mathrm{C}$ and the resulting yellow mixture was stirred $30 \mathrm{~min}$. (Trimethylsilyl)(triisopropylsilyl)acetylene (15) (2.00 g, $7.86 \mathrm{mmol}, 1.00$ equiv) was added and the mixture was then stirred $2 \mathrm{~h}$. Water was then added $(30 \mathrm{~mL})$ followed by extraction of the aqueous layer with dichloromethane $(2 \times 30 \mathrm{~mL})$. The combined organic layers were dried over $\mathrm{MgSO}_{4}$, filtered and the solvent was evaporated under reduced pressure. The resulting solid was triturated in hexane $(10 \mathrm{~mL})$. Filtration and removal of solvent in vacuo afforded phenyl(triisopropylsilyl)iodonium triflate (20) (2.90 g, $11.2 \mathrm{mmol}, 70 \%$ yield) as a colorless solid. ${ }^{1} \mathbf{H}-\mathbf{N M R}\left(400 \mathrm{MHz}, \mathrm{CDCl}_{3}\right)$ $\delta=8.09(\mathrm{~m}, 2 \mathrm{H}), 7.65(\mathrm{~m}, 1 \mathrm{H}), 7.52(\mathrm{~m}, 2 \mathrm{H}), 1.15-1.01(\mathrm{~m}, 21 \mathrm{H})$ ppm. ${ }^{13} \mathbf{C}\left\{{ }^{1} \mathbf{H}\right\}-\mathbf{N M R}\left(100 \mathrm{MHz}, \mathrm{CDCl}_{3}\right)$ 
$\delta=133.7,132.5,132.4,119.7,117.6,117.6,44.9,18.3,11.1 \mathrm{ppm}$. The values of the NMR spectra are in accordance with reported literature data. ${ }^{36}$

\section{Preparation of Starting Materials}

All thiols were commercially avaiable and purhased from Sigma-Aldrich, Alfa-Aesar, Fluorochem or TCI and used as purchased without any purification.

\section{General procedure B: synthesis of tert-butyl $\beta$-sulfanyl esters $(21 \mathrm{a}-21 \mathrm{k})$}

Following a slightly modified version of a reported procedure, ${ }^{37}$ the thiol (1 equiv.), tert-butyl acrylate (1.05 equiv.) and triethylamine (0.1 equiv.) were introduced into a microwave vial equipped with a magnetic stirrer. The vial was sealed and the reaction mixture was stirred at $\mathrm{rt}$ for 10 minutes then heated to $60{ }^{\circ} \mathrm{C}$ for 1-3 hours (until full conversion based on TLC analysis). The volatiles were then removed through evaporation under reduced pressure to afford $\beta$-sulfanyl esters $(\mathbf{2 1 a - 2 1 k})$ in sufficient purity to be used in next step without further purification.

In order to characterise the new compounds, preparative TLC $\left(\mathrm{SiO}_{2}, \mathrm{Heptane} / \mathrm{EtOAc}\right)$ was used to afford pure compounds.

\section{General procedure C: synthesis of tert-butyl $\beta$-sulfanyl esters $(211,21 \mathrm{~m})$}

Following a reported procedure, ${ }^{18 \mathrm{e}}$ the thiol (1.0 equiv.) and tert-butyl acrylate (1.0 equiv.) were dissolved in THF ( $5 \mathrm{M}$ based on the thiol) in a round-bottomed flask equipped with a magnetic stirrer. Triethylamine (0.1 equiv.) was added and the reaction mixture was stirred at rt. overnight. It was then concentrated under reduced pressure to remove the volatiles. The crude was then submitted to column chromatography $\left(\mathrm{SiO}_{2}\right.$, pentane:Et $2 \mathrm{O})$ affording $\beta$-sulfanyl esters $(\mathbf{2 1 1}, \mathbf{2 1} \mathbf{m})$.

\section{General alkylation procedure D: synthesis of tert-butyl $\beta$-sulfanyl esters $(21 \mathrm{n}, 210)$}

Following a slightly modified reported procedure, ${ }^{38}$ the thiol (1.0 equiv.) was added to a suspension of $\mathrm{KF} /$ alumina $45 \mathrm{wt} \%$ (4.2 equiv -4.6 equiv) in acetonitrile $(0.05 \mathrm{M}$ based on the thiol). The reaction mixture was stirred for 5 minutes at rt. tert-Butyl acrylate (1.0 equiv.) was added and the reaction was heated 
to $50-60{ }^{\circ} \mathrm{C}$ for 15 minutes. The reaction mixture was filtered on a Celite pad then concentrated under reduced pressure and filtered a second time on Celite and sand, washed with DCM. Solvents were evaporated under reduced pressure affording in sufficient purity the desired tert-butyl alkylthiopropanoate (21n, 210).

\section{Synthesis of alumina supported potassium fluoride $45 \mathrm{wt} \%$}

Following a reported procedure, ${ }^{38}$ basic alumina $(5.5 \mathrm{~g})$ and potassium fluoride $(4.5 \mathrm{~g}, 77 \mathrm{mmol})$ were charged in a $25 \mathrm{~mL}$ flask equipped with a magnetic stirrer. Water $(10 \mathrm{~mL})$ was added and the mixture was stirred at room temperature for $15 \mathrm{~min}$. The water was evaporated under reduced pressure and the catalyst was left to dry in a vacuum oven at $110^{\circ} \mathrm{C}$ overnight, yielding $10 \mathrm{~g} 45 \mathrm{wt} \% \mathrm{KF} / \mathrm{b}$ asic alumina support.

\section{Tert-butyl 3-(4-methylphenylthio)propanoate (21a)}

1. ${ }^{\mathrm{s}} \sim_{\mathrm{CO}_{2} \mathrm{Bu}}$ Following general procedure B, 21a was obtained as a yellow oil from 4-methylbenzenethiol (1.24 g, $10.0 \mathrm{mmol})$ and tert-butyl acrylate $(1.5 \mathrm{~mL}, 10 \mathrm{mmol})$. Yield: 99\% (2.51 g, $9.95 \mathrm{mmol}$, 88\% purity). ${ }^{1} \mathbf{H}-\mathbf{N M R}\left(400 \mathrm{MHz}, \mathrm{CDCl}_{3}\right) \delta 7.28(\mathrm{~m}, 2 \mathrm{H}), 7.11(\mathrm{~m}, 2 \mathrm{H}), 3.07(\mathrm{t}, J=7.5 \mathrm{~Hz}, 2 \mathrm{H}), 2.51$ (t, $J=7.5 \mathrm{~Hz}, 2 \mathrm{H}), 2.32(\mathrm{~s}, 3 \mathrm{H}), 1.45(\mathrm{~s}, 9 \mathrm{H}) \mathrm{ppm} .{ }^{13} \mathbf{C}\left\{{ }^{1} \mathbf{H}\right\}-\mathbf{N M R}\left(101 \mathrm{MHz}, \mathrm{CDCl}_{3}\right) \delta=171.2,136.8$, $131.8,131.0,129.9,80.9,35.8,30.1,28.2,21.2 \mathrm{ppm}$. The values of the NMR spectra are in accordance with reported literature data. ${ }^{39}$

\section{Tert-butyl 3-(phenylthio)propanoate (21b)}

$$
\begin{aligned}
& \text { (0.73 mL, } 7.1 \mathrm{mmol}) \text { and tert-butyl acrylate }(1.1 \mathrm{~mL}, 7.5 \mathrm{mmol}) . \text { Yield: } 99 \%(1.68 \mathrm{~g}, 7.10 \mathrm{mmol}) .{ }^{1} \mathbf{H}- \\
& \text { NMR }\left(400 \mathrm{MHz}, \mathrm{CDCl}_{3}\right) \delta=7.39-7.33(\mathrm{~m}, 2 \mathrm{H}), 7.33-7.26(\mathrm{~m}, 2 \mathrm{H}), 7.24-7.17(\mathrm{~m}, 1 \mathrm{H}), 3.13(\mathrm{dd}, J \\
& =7.9,7.1 \mathrm{~Hz}, 2 \mathrm{H}), 2.54(\mathrm{dd}, J=7.8,7.1 \mathrm{~Hz}, 2 \mathrm{H}), 1.45(\mathrm{~s}, 9 \mathrm{H}) \mathrm{ppm} .{ }^{13} \mathbf{C}\left\{{ }^{1} \mathbf{H}\right\}-\mathrm{NMR}\left(101 \mathrm{MHz}, \mathrm{CDCl}_{3}\right) \\
& \delta=171.1,135.6,130.0,129.0,126.4,81.0,35.6,29.2,28.1 \mathrm{ppm} . \mathbf{H R M S}(\mathrm{APCI}) \text { calcd. For } \mathrm{C}_{13} \mathrm{H}_{18} \mathrm{O}_{2} \mathrm{~S}^{+{ }^{\circ}} \\
& {[\mathrm{M}]^{+{ }^{\circ}} 238.1028 ; \text { found } 238.1022 . \mathbf{I R}(\mathrm{film}): \widetilde{v}=2977(\mathrm{~m}), 2935(\mathrm{w}), 1729(\mathrm{~s}), 1480(\mathrm{~m}), 1368(\mathrm{~m}), 1249} \\
& (\mathrm{~m}), 1152(\mathrm{~s}) \mathrm{cm}^{-1} .
\end{aligned}
$$


$\mathrm{MeO}$ ybenzenethiol $(0.44 \mathrm{~mL}, 3.6 \mathrm{mmol})$ and tert-butyl acrylate $(0.54 \mathrm{~mL}, 3.7 \mathrm{mmol})$. Yield: $99 \%$ (0.954 g, $3.55 \mathrm{mmol}) .{ }^{1} \mathbf{H}-\mathbf{N M R}\left(400 \mathrm{MHz}, \mathrm{CDCl}_{3}\right) \delta=7.37(\mathrm{dt}, J=8.8,2.1 \mathrm{~Hz}, 2 \mathrm{H}), 6.84(\mathrm{dt}, J=8.8,2.1 \mathrm{~Hz}$, 2H), $3.80(\mathrm{~s}, 3 \mathrm{H}), 3.00(\mathrm{t}, J=7.5 \mathrm{~Hz}, 2 \mathrm{H}), 2.47(\mathrm{t}, J=7.5 \mathrm{~Hz}, 2 \mathrm{H}), 1.44(\mathrm{~s}, 9 \mathrm{H}) \mathrm{ppm} .{ }^{13} \mathbf{C}\left\{{ }^{1} \mathbf{H}\right\}-\mathbf{N M R}$ $\left(101 \mathrm{MHz}^{\left.\mathrm{CDCl}_{3}\right)} \delta=171.3,159.4,134.2,125.5,114.7,81.0,55.5,35.8,31.4,28.2\right.$ ppm. HRMS (ESI) calcd. for $\mathrm{C}_{14} \mathrm{H}_{20} \mathrm{NaO}_{3} \mathrm{~S}^{+}[\mathrm{M}+\mathrm{Na}]^{+}$291.1025; found 291.1034. IR (film): $\widetilde{v}=2979$ (w), 2934 (w), 2840 (w), $1727(\mathrm{~s}), 1596(\mathrm{~m}), 1579(\mathrm{w}), 1497(\mathrm{~m}), 1368(\mathrm{~m}), 1304(\mathrm{w}), 1250(\mathrm{~s}), 1155(\mathrm{~s}), 1089(\mathrm{~s}), 1043$ (s), $834(\mathrm{~m}) \mathrm{cm}^{-1}$.

\section{Tert-butyl 3-(4-bromophenylthio)propanoate (21d)}

Following general procedure B, 21d was obtained as an off-white solid from 4-bromobenzenethiol $(0.510 \mathrm{~g}, 2.70 \mathrm{mmol})$ and tert-butyl acrylate $(0.4 \mathrm{~mL}, 3 \mathrm{mmol})$. Yield 96\% (0.827 g, 2.61 mmol). mp: $46.8-47.9{ }^{\circ} \mathrm{C} ;{ }^{1} \mathbf{H}-\mathbf{N M R}\left(400 \mathrm{MHz}, \mathrm{CDCl}_{3}\right) \delta=7.41(\mathrm{dt}, J=8.5,2.0 \mathrm{~Hz}, 2 \mathrm{H}), 7.22(\mathrm{dt}, J=$ 8.5, 2.0 Hz, 2H), $3.10(\mathrm{t}, J=7.4 \mathrm{~Hz}, 2 \mathrm{H}), 2.52(\mathrm{t}, J=7.4 \mathrm{~Hz}, 2 \mathrm{H}), 1.45(\mathrm{~s}, 9 \mathrm{H}) \mathrm{ppm} .{ }^{13} \mathbf{C}\left\{{ }^{1} \mathbf{H}\right\}-\mathbf{N M R}(101$ $\left.\mathrm{MHz}_{\mathrm{CDCl}}\right) \delta=171.0,134.9,132.2,131.6,120.4,81.3,35.5,29.4,28.2$ ppm. HRMS (ESI) calcd. for $\mathrm{C}_{13} \mathrm{H}_{17}{ }^{79} \mathrm{BrNaO}_{2} \mathrm{~S}^{+}[\mathrm{M}+\mathrm{Na}]^{+}$339.0030; found 339.0029. IR (film): $\widetilde{v}=2979(\mathrm{w}), 2938(\mathrm{w}), 1729$ (s), 1476 (m), 1391 (w), 1367 (m), 1252 (m), 1156 (s), 1094 (w), 1009 (w), 814 (w) cm cm $^{-1}$

\section{Tert-butyl 3-(4-fluorophenylthio)propanoate (21e)}

Following general procedure B, 21e was obtained as a yellow oil from 4-fluorobenzenethiol $(0.42 \mathrm{~mL}, 3.9 \mathrm{mmol})$ and tert-butyl acrylate $(0.64 \mathrm{~mL}, 4.4 \mathrm{mmol})$. Yield: 92\% (95\% pure according to NMR analysis; $0.97 \mathrm{~g}, 3.6 \mathrm{mmol}) .{ }^{1} \mathbf{H}-\mathbf{N M R}\left(400 \mathrm{MHz}, \mathrm{CDCl}_{3}\right) \delta=7.38(\mathrm{~m}, 2 \mathrm{H}), 7.12-6.72$ $(\mathrm{m}, 2 \mathrm{H}), 3.06(\mathrm{t}, J=7.4 \mathrm{~Hz}, 2 \mathrm{H}), 2.49(\mathrm{t}, J=7.4 \mathrm{~Hz}, 2 \mathrm{H}), 1.44(\mathrm{~s}, 9 \mathrm{H}) \mathrm{ppm} .{ }^{13} \mathbf{C}\left\{{ }^{\mathbf{1}} \mathbf{H}\right\}-\mathbf{N M R}(101 \mathrm{MHz}$, $\left.\mathrm{CDCl}_{3}\right) \delta=171.0,162.1(\mathrm{~d}, J=246.8 \mathrm{~Hz}), 133.2(\mathrm{~d}, J=8.1 \mathrm{~Hz}), 130.3(\mathrm{~d}, J=3.4 \mathrm{~Hz}), 116.1(\mathrm{~d}, J=21.8$ $\mathrm{Hz}), 81.0,35.5,30.6,28.1 \mathrm{ppm} .{ }^{19} \mathbf{F}$ NMR $\left(376 \mathrm{MHz}, \mathrm{CDCl}_{3}\right) \delta=-115.0 \mathrm{ppm}$. HRMS (ESI) calcd. for 
$\mathrm{C}_{13} \mathrm{H}_{17} \mathrm{FNaO}_{2} \mathrm{~S}^{+}[\mathrm{M}+\mathrm{Na}]^{+}$279.0825; found 279.0817. IR (film): $\widetilde{v}=2983(\mathrm{~m}), 2923(\mathrm{w}), 1728(\mathrm{~s}), 1591$

(m), $1489(\mathrm{~s}), 1368(\mathrm{~m}), 1230(\mathrm{~s}), 1153(\mathrm{~s}), 828(\mathrm{~s}) \mathrm{cm}^{-1}$.

\section{Tert-butyl 3-(4-trifluoromethylphenylthio)propanoate (21f)}

Following general procedure B, 21f was obtained as a pale yellow oul from 4-trifluoromethylbenzenethiol $(0.30 \mathrm{~mL}, 2.2 \mathrm{mmol})$ and tert-butyl acrylate $(0.36 \mathrm{~mL}, 2.5 \mathrm{mmol})$. Yield: $99 \%$ (0.665 g, $2.17 \mathrm{mmol}) .{ }^{1} \mathbf{H}-\mathbf{N M R}\left(400 \mathrm{MHz}, \mathrm{CDCl}_{3}\right) \delta=7.52(\mathrm{~m}, 2 \mathrm{H}), 7.38(\mathrm{~m}, 2 \mathrm{H}), 3.20(\mathrm{t}, J=7.4 \mathrm{~Hz}$, 2H ), $2.58(\mathrm{dd}, J=7.7,7.1 \mathrm{~Hz}, 2 \mathrm{H}), 1.45(\mathrm{~s}, 9 \mathrm{H}) \mathrm{ppm} .{ }^{13} \mathbf{C}\left\{{ }^{1} \mathbf{H}\right\}-\mathbf{N M R}\left(101 \mathrm{MHz}, \mathrm{CDCl}_{3}\right) \delta=170.7$, $141.4,128.0,127.8(\mathrm{q}, J=32.8 \mathrm{~Hz}), 125.8(\mathrm{q}, J=3.7 \mathrm{~Hz}), 124.2(\mathrm{q}, J=271.7 \mathrm{~Hz}), 81.3,35.1,28.1,27.9$ ppm. ${ }^{19} \mathbf{F}$ NMR $\left(376 \mathrm{MHz}, \mathrm{CDCl}_{3}\right) \delta=-62.9$ ppm. HRMS (ESI) calcd. for $\mathrm{C}_{14} \mathrm{H}_{17} \mathrm{~F}_{3} \mathrm{O}_{2} \mathrm{~S}^{+}[\mathrm{M}]^{+}$306.0896; found 306.0902. IR (film): $\widetilde{v}=2977$ (w), 2935 (w), 1728 (m), 1608 (m), 1370 (m), 1327 (s), 1166 (s), 1127 (s), 1097 (s), $1065(\mathrm{~m}), 1014(\mathrm{~m}) \mathrm{cm}^{-1}$.

\section{Tert-butyl 3-(4-nitrophenylthio)propanoate (21g)}

Following general procedure B, 21g was obtained as a yellow crystalline solid from 4-nitrobenzenethiol $(0.50 \mathrm{~g}, 3.2 \mathrm{mmol})$ and tert-butyl acrylate $(0.56 \mathrm{~mL}, 3.9 \mathrm{mmol})$. Yield: $55 \%$ (0.50 g, $1.8 \mathrm{mmol}) . \mathbf{m p :} 70.1-71.8{ }^{\circ} \mathrm{C} .{ }^{1} \mathbf{H}-\mathbf{N M R}\left(400 \mathrm{MHz}, \mathrm{CDCl}_{3}\right) \delta=8.14(\mathrm{~m}, 2 \mathrm{H}), 7.35(\mathrm{~m}, 2 \mathrm{H}), 3.26(\mathrm{t}, J=$ $7.4 \mathrm{~Hz}, 2 \mathrm{H}), 2.62(\mathrm{t}, J=7.3 \mathrm{~Hz}, 2 \mathrm{H}), 1.46(\mathrm{~s}, 9 \mathrm{H}) \mathrm{ppm} .{ }^{13} \mathbf{C}\left\{{ }^{1} \mathbf{H}\right\}-\mathbf{N M R}\left(101 \mathrm{MHz}, \mathrm{CDCl}_{3}\right) \delta=170.4$, 146.7, 145.2, 126.5, 124.1, 81.6, 34.7, 28.1, 27.2 ppm. HRMS (ESI) calcd. for $\mathrm{C}_{13} \mathrm{H}_{17} \mathrm{NNaO}_{4} \mathrm{~S}^{+}$ $[\mathrm{M}+\mathrm{Na}]^{+}$306.0770; found 306.0761. IR (film): $\widetilde{v}=3094(\mathrm{w}), 3072(\mathrm{w}), 3002(\mathrm{w}), 2970(\mathrm{w}), 2932(\mathrm{w})$, $1731(\mathrm{~s}), 1591(\mathrm{~m}), 1581(\mathrm{~m}), 1503(\mathrm{~s}), 1336(\mathrm{~s}), 1225(\mathrm{~m}), 1153(\mathrm{~s}), 1089(\mathrm{~s}), 855(\mathrm{~m}), 836(\mathrm{~s}), 744(\mathrm{~m})$ $\mathrm{cm}^{-1}$.

\section{Tert-butyl 3-(2-bromophenylthio)propanoate (21h)}

Following general procedure B, 21h was obtained as a yellow oil from 2-bromobenzenethiol $(0.31 \mathrm{~mL}, 2.6 \mathrm{mmol})$ and tert-butyl acrylate $(0.46 \mathrm{~mL}, 3.2 \mathrm{mmol})$. Yield: 99\% (0.89 g, 2.6 mmol). ${ }^{1} \mathbf{H}-\mathbf{N M R}\left(400 \mathrm{MHz}, \mathrm{CDCl}_{3}\right) \delta=7.51(\mathrm{dd}, J=7.9,1.2 \mathrm{~Hz}, 1 \mathrm{H}), 7.30-7.19(\mathrm{~m}, 2 \mathrm{H}), 7.01$ (ddd, 
$J=7.9,6.6,2.3 \mathrm{~Hz}, 1 \mathrm{H}), 3.13(\mathrm{t}, J=7.5 \mathrm{~Hz}, 2 \mathrm{H}), 2.55(\mathrm{t}, J=7.5 \mathrm{~Hz}, 2 \mathrm{H}), 1.43(\mathrm{~s}, 9 \mathrm{H}) \mathrm{ppm} .{ }^{13} \mathbf{C}\left\{{ }^{1} \mathbf{H}\right\}-$ $\operatorname{NMR}\left(101 \mathrm{MHz}, \mathrm{CDCl}_{3}\right) \delta=170.8,137.2,133.1,128.7,127.8,127.0,124.2,81.2,34.9,28.2,28.1 \mathrm{ppm}$. HRMS (ESI) calcd. for $\mathrm{C}_{13} \mathrm{H}_{17}{ }^{79} \mathrm{BrNaO}_{2} \mathrm{~S}^{+}[\mathrm{M}+\mathrm{Na}]^{+}$339.0025; found 339.0017. IR (film): $\widetilde{v}=3002(\mathrm{w}$ ), 2976 (w), 2932 (w), 1725 (s), 1582 (w), 1449 (m), 1427 (m), 1367 (m), 1251 (m), 1147 (s), 1021 (m), $838(\mathrm{~m}), 744(\mathrm{~s}) \mathrm{cm}^{-1}$.

\section{Tert-butyl 3-(3-methylphenylthio)propanoate (21i)}

Me ${ }^{s} \backsim \mathrm{CO}_{2} \mathrm{Bu}^{\mathrm{Bu}}$ Following general procedure B, 21i was obtained as a pale yellow oil from 3methylbenzenethiol $(1.00 \mathrm{~mL}, 8.4 \mathrm{mmol})$ and tert-butyl acrylate $(1.3 \mathrm{~mL}, 9.0 \mathrm{mmol})$. Yield: $99 \%(2.1 \mathrm{~g}$, $8.3 \mathrm{mmol}) .{ }^{1} \mathrm{H}-\mathrm{NMR}\left(400 \mathrm{MHz}, \mathrm{CDCl}_{3}\right) \delta=7.19-7.13(\mathrm{~m}, 3 \mathrm{H}), 6.96(\mathrm{~m}, 1 \mathrm{H}), 3.10(\mathrm{t}, J=7.5 \mathrm{~Hz}, 2 \mathrm{H})$, $2.52(\mathrm{t}, J=7.5 \mathrm{~Hz}, 2 \mathrm{H}), 2.30(\mathrm{~s}, 3 \mathrm{H}), 1.44(\mathrm{~s}, 9 \mathrm{H}) \mathrm{ppm} .{ }^{13} \mathbf{C}\left\{{ }^{1} \mathbf{H}\right\}-\mathbf{N M R}\left(101 \mathrm{MHz}, \mathrm{CDCl}_{3}\right) \delta=171.0$, 138.6, 135.3, 130.5, 128.8, 127.2, 126.9, 80.8, 35.5, 29.1, 28.0, 21.3 ppm. HRMS (APPI) calcd. for $\mathrm{C}_{14} \mathrm{H}_{20} \mathrm{O}_{2} \mathrm{~S}^{+\circ}[\mathrm{M}]^{+\circ}$ 252.1179; found 252.1174. IR (film): $\widetilde{v}=3008$ (w), 2976 (w), 2932 (w), 1726 (s), $1595(\mathrm{w}), 1472(\mathrm{w}), 1364(\mathrm{~m}), 1248(\mathrm{~m}), 1149(\mathrm{~s}), 844(\mathrm{~m}), 771(\mathrm{~s}) \mathrm{cm}^{-1}$.

\section{Tert-butyl 3-(3-bromophenylthio)propanoate (21j)}

${ }^{\mathrm{Br}} \mathrm{C}^{\mathrm{S}} \sim \mathrm{CO}_{2} \mathrm{Bu}$ Following general procedure $\mathbf{B}, \mathbf{2 1} \mathbf{j}$ was obtained as a pale yellow oil from 3-bromobenzenethiol $(0.30 \mathrm{~mL}, 2.6 \mathrm{mmol})$ and tert-butyl acrylate $(0.4 \mathrm{~mL}, 3 \mathrm{mmol})$. Yield: $99 \%(0.82 \mathrm{~g}, 2.6$ mmol). ${ }^{1} \mathbf{H}-\mathrm{NMR}\left(400 \mathrm{MHz}, \mathrm{CDCl}_{3}\right) \delta=7.48(\mathrm{t}, J=1.8 \mathrm{~Hz}, 1 \mathrm{H}), 7.32(\mathrm{ddd}, J=7.9,1.9,1.0 \mathrm{~Hz}, 1 \mathrm{H})$, 7.26 (ddd, $J=7.9,1.8,1.0 \mathrm{~Hz}, 1 \mathrm{H}), 7.15(\mathrm{t}, J=7.9 \mathrm{~Hz}, 1 \mathrm{H}), 3.13(\mathrm{t}, J=7.4 \mathrm{~Hz}, 2 \mathrm{H}), 2.54$ (t, $J=7.4 \mathrm{~Hz}$, 2H), 1.45 (s, 9H) ppm. ${ }^{13} \mathbf{C}\left\{{ }^{1} \mathbf{H}\right\}-N M R\left(101 \mathrm{MHz}, \mathrm{CDCl}_{3}\right) \delta=170.8,138.2,131.8,130.3,129.3,127.9$, 122.9, 81.2, 35.3, 28.9, 28.1 ppm. HRMS (APPI) calcd. for $\mathrm{C}_{13} \mathrm{H}_{17}{ }^{79} \mathrm{BrO}_{2} \mathrm{~S}^{+\circ}[\mathrm{M}]^{+\circ} 316.0127$; found 316.0132. IR (film): $\widetilde{v}=2977$ (m), 2923 (w), 1728 (s), 1576 (m), 1557 (m), 1459 (m), 1391 (m), 1370 (m), $1247(\mathrm{~m}), 1152(\mathrm{~s}), 844(\mathrm{~m})$.

\section{Tert-butyl 3-(pyridin-2-ylthio)propanoate (21k)}




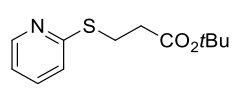

Following slightly modified general procedure B, 21k was obtained as a dark brown oil from pyridine-2-thiol $(0.82 \mathrm{~g}, 7.4 \mathrm{mmol})$ and tert-butyl acrylate $(1.2 \mathrm{~mL}, 7.8 \mathrm{mmol})$ after heating to $80{ }^{\circ} \mathrm{C}$ over 16 hours. Yield: $90 \%$ (90\% pure according to NMR analysis; $\left.1.75 \mathrm{~g}, 6.64 \mathrm{mmol}\right)$. ${ }^{1} \mathbf{H}-\mathbf{N M R}\left(400 \mathrm{MHz}, \mathrm{CDCl}_{3}\right) \delta=8.42(\mathrm{ddd}, J=5.0,1.9,1.0 \mathrm{~Hz}, 1 \mathrm{H}), 7.46(\mathrm{ddd}, J=8.0,7.4,1.9 \mathrm{~Hz}, 1 \mathrm{H})$, $7.16(\mathrm{dt}, J=8.1,1.1 \mathrm{~Hz}, 1 \mathrm{H}), 6.96(\mathrm{ddd}, J=7.4,4.9,1.1 \mathrm{~Hz}, 1 \mathrm{H}), 3.39(\mathrm{t}, J=7.1 \mathrm{~Hz}, 2 \mathrm{H}), 2.68(\mathrm{t}, J=7.1$ $\mathrm{Hz}, 2 \mathrm{H}), 1.46$ (s, 9H) ppm. ${ }^{13} \mathbf{C}\left\{{ }^{1} \mathbf{H}\right\}-\mathbf{N M R}\left(101 \mathrm{MHz}, \mathrm{CDCl}_{3}\right) \delta=171.4,158.5,149.5,135.9,122.3$, 119.4, 80.8, 35.8, 28.1, 25.1 ppm. HRMS (ESI) calcd. for $\mathrm{C}_{12} \mathrm{H}_{18} \mathrm{NO}_{2} \mathrm{~S}^{+}[\mathrm{M}+\mathrm{H}]^{+}$240.1053; found 240.1047. IR (film): $\widetilde{v}=3049$ (w), 2977 (w), 2935 (w), 1728 (s), 1580 (m), 1560 (w), 1534 (w), 1457 (m), $1418(\mathrm{~s}), 1367(\mathrm{~s}), 1285(\mathrm{~m}), 1254(\mathrm{~s}), 1151(\mathrm{~s}), 1130(\mathrm{~s}) \mathrm{cm}^{-1}$.

\section{Tert-butyl 3-((thiophen-2-yl)thio)propanoate (211)}

${ }^{\mathrm{s}} \mathrm{S}^{\mathrm{s}} \sim_{\mathrm{CO}_{2} \mathrm{Bu}}$ Following general procedure $\mathbf{C}, \mathbf{2 1 1}$ was obtained as a yellow oil from thiophenethiol (1.0 mL, $11 \mathrm{mmol}, 1.0$ equiv.) and tert-butyl acrylate (1.6 mL, $11 \mathrm{mmol}, 1.0$ equiv.). Yield: $70 \%$ (1.83 g, $7.48 \mathrm{mmol}) .{ }^{1} \mathrm{H}-\mathrm{NMR}\left(400 \mathrm{MHz}, \mathrm{CDCl}_{3}\right) \delta=7.36(\mathrm{dd}, J=5.4,1.2 \mathrm{~Hz}, 1 \mathrm{H}), 7.14(\mathrm{dd}, J=3.6,1.2 \mathrm{~Hz}$, 1H), 6.98 (dd, $J=5.4,3.6 \mathrm{~Hz}, 1 \mathrm{H}), 2.97$ (t, $J=7.3 \mathrm{~Hz}, 2 \mathrm{H}), 2.53$ (t, $J=7.4 \mathrm{~Hz}, 2 \mathrm{H}), 1.45$ (s, 9H) ppm. ${ }^{13} \mathbf{C}\{\mathbf{1 H}\}-N M R\left(101 \mathrm{MHz}, \mathrm{CDCl}_{3}\right) \delta=170.1,145.3,131.2,129.7,127.5,81.7,53.0,28.2,28.0 \mathrm{ppm}$. HRMS (ESI) calcd. for $\mathrm{C}_{11} \mathrm{H}_{16} \mathrm{NaO}_{2} \mathrm{~S}_{2}{ }^{+}[\mathrm{M}+\mathrm{Na}]^{+}$267.0484; found 267.0474. IR (film): $\widetilde{v}=2974$ (m), $2928(\mathrm{w}), 1725(\mathrm{~s}), 1407$ (w), $1366(\mathrm{~m}), 1247$ (m), 1143 (s), $991(\mathrm{w}), 846(\mathrm{~s}) \mathrm{cm}^{-1}$.

\section{Tert-butyl 3-((2-methylfuran-3-yl)thio)propanoate (21 m)}

Me ran-3-thiol $(0.50 \mathrm{~mL}, 4.5 \mathrm{mmol})$ and tert-butyl acrylate $(0.66 \mathrm{~mL}, 4.5 \mathrm{mmol})$. Yield: $76 \%(0.828 \mathrm{~g}, 3.42$ mmol). ${ }^{1} \mathbf{H}-\mathbf{N M R}\left(400 \mathrm{MHz}, \mathrm{CDCl}_{3}\right) \delta=7.28(\mathrm{~d}, J=2.0 \mathrm{~Hz}, 1 \mathrm{H}), 6.34(\mathrm{~d}, J=2.0 \mathrm{~Hz}, 1 \mathrm{H}), 2.81(\mathrm{t}, J=$ $7.3 \mathrm{~Hz}, 2 \mathrm{H}), 2.43(\mathrm{t}, J=7.3 \mathrm{~Hz}, 2 \mathrm{H}), 2.34(\mathrm{~s}, 3 \mathrm{H}), 1.44(\mathrm{~s}, 9 \mathrm{H}) \mathrm{ppm} .{ }^{13} \mathbf{C}\left\{{ }^{1} \mathbf{H}\right\}-\mathbf{N M R}\left(101 \mathrm{MHz}, \mathrm{CDCl}_{3}\right)$ $\delta=171.2,155.4,140.7,115.1,109.5,80.8,35.9,31.1,28.1,11.8$ ppm. HRMS (ESI) calcd. for 
$\mathrm{C}_{12} \mathrm{H}_{18} \mathrm{NaO}_{3} \mathrm{~S}^{+}[\mathrm{M}+\mathrm{Na}]^{+}$265.0869; found 265.0858. IR (film): $\widetilde{v}=2979$ (m), 2934 (w), 1730 (s), 1514

(w), $1370(\mathrm{~m}), 1253(\mathrm{~m}), 1142(\mathrm{~s}), 1091(\mathrm{~m}), 935$ (m), $850(\mathrm{~m}), 739(\mathrm{~m}) \mathrm{cm}^{-1}$.

\section{Tert-butyl 3-(pentylthio)propanoate (21n)}

$\sim \sim_{\mathrm{CO}_{2} \mathrm{tBu}}$ Following general procedure D, 21n was obtained as a yellow oil from 1-pentanethiol (1.24 g, $10.0 \mathrm{mmol}, 1.0$ equiv.), KF/alumina 45wt\% (6.0 g, $42 \mathrm{mmol}, 4.2$ equiv.) and tert-butyl acrylate (1.5 mL, 10 mmol, 1 equiv.).Yield: $99 \%(2.51 \mathrm{~g}, 10.0 \mathrm{mmol}) .{ }^{1} \mathbf{H}-\mathbf{N M R}\left(400 \mathrm{MHz}, \mathrm{CDCl}_{3}\right) \delta=2.73(\mathrm{t}, J$ $=7.4 \mathrm{~Hz}, 2 \mathrm{H}), 2.59-2.43(\mathrm{~m}, 4 \mathrm{H}), 1.65-1.49(\mathrm{~m}, 2 \mathrm{H}), 1.45(\mathrm{~s}, 9 \mathrm{H}), 1.40-1.26(\mathrm{~m}, 4 \mathrm{H}), 0.95-0.82(\mathrm{~m}, 3 \mathrm{H}$, ppm. ${ }^{13} \mathbf{C}\left\{{ }^{1} \mathbf{H}\right\}$-NMR $\left(101 \mathrm{MHz}, \mathrm{CDCl}_{3}\right) \delta=171.5,80.9,36.3,32.2,31.2,29.4,28.2,27.3,22.5,14.1$ ppm. HRMS (ESI) calcd. for $\mathrm{C}_{12} \mathrm{H}_{24} \mathrm{NaO}_{2} \mathrm{~S}^{+}[\mathrm{M}+\mathrm{Na}]^{+}$255.1389; found 255.1394. IR (film): $\widetilde{v}=2960$ (m), 2929 (m), $2861(\mathrm{w}), 1731$ (s), $1462(\mathrm{w}), 1392(\mathrm{w}), 1371(\mathrm{w}), 1251(\mathrm{~m}), 1145$ (s), $971(\mathrm{w}), 934(\mathrm{w})$, $849(\mathrm{w}) \mathrm{cm}^{-1}$.

\section{Tert-butyl 3-(cyclohexylthio)propanoate (210)}

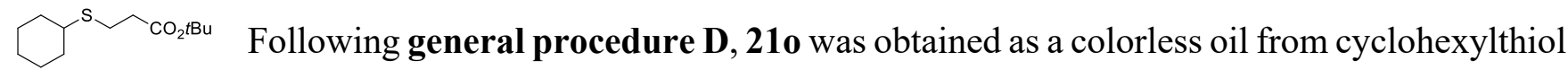
(0.73 mL, $6.0 \mathrm{mmol}, 1.0$ equiv.), KF/alumina 45wt\% (3.6 g, $25 \mathrm{mmol}, 4.2$ equiv.) and tert-butyl acrylate (0.90 mL, $6.2 \mathrm{mmol}, 1.05$ equiv.). ${ }^{1} \mathrm{H}-\mathbf{N M R}\left(400 \mathrm{MHz}, \mathrm{CDCl}_{3}\right) \delta=2.75(\mathrm{dd}, J=8.0,7.1 \mathrm{~Hz}, 2 \mathrm{H}), 2.64$ (tt, $J=10.4,3.6 \mathrm{~Hz}, 1 \mathrm{H}), 2.49(\mathrm{dd}, J=8.1,7.1 \mathrm{~Hz}, 2 \mathrm{H}), 2.01-1.89(\mathrm{~m}, 2 \mathrm{H}), 1.91-1.72(\mathrm{~m}, 2 \mathrm{H}), 1.65-$ $1.55(\mathrm{~m}, 1 \mathrm{H}), 1.45(\mathrm{~s}, 9 \mathrm{H}), 1.38-1.17(\mathrm{~m}, 5 \mathrm{H}) \mathrm{ppm} .{ }^{13} \mathbf{C}\left\{{ }^{1} \mathbf{H}\right\}-\mathbf{N M R}\left(101 \mathrm{MHz}, \mathrm{CDCl}_{3}\right) \delta=171.4,80.7$ 43.6, 36.5, 33.7, 28.1, 26.1, 25.8, 25.3 ppm. HRMS (ESI) calcd. for $\mathrm{C}_{13} \mathrm{H}_{24} \mathrm{NaO}_{2} \mathrm{~S}^{+}[\mathrm{M}+\mathrm{Na}]^{+}$267.1389; found 267.1390. IR (film): $\widetilde{v}=2981$ (m), 2929 (s), 2857 (m), 1732 (s), 1447 (m), 1370 (s), 1250 (s), 1155 (s) $\mathrm{cm}^{-1}$.

\section{General oxidation procedure E (compounds 5, 6a-6j, 6n and 8)}

Following a slightly modified version of a reported procedure, ${ }^{16}$ in a two-necked flask equipped with a magnetic stirrer, the sulfide 21 ( 1 equiv.) was dissolved in methanol (40 mL) and cooled to $0{ }^{\circ} \mathrm{C}$. Sodium periodate (1.0 equiv. or 1.2 equiv.) dissolved in water $(20 \mathrm{~mL})$ was then added dropwise using a syringe 
over a period of 30 minutes. The reaction mixture was then left to warm to room temperature under stirring overnight. The precipitated solids were then filtered off through a Celite pad and washed with methanol $(20 \mathrm{~mL})$. Methanol was evaporated under reduced pressure. The obtained aqueous layer was extracted three times with DCM $(3 \times 20 \mathrm{~mL})$. The organic layers were combined, washed with brine $(10 \mathrm{~mL})$, dried over $\mathrm{Na}_{2} \mathrm{SO}_{4}$ and filtered. Solvents were then evaporated under reduced pressure before submitting the crude to column chromatography (silica gel, DCM/EtOAc) affording the pure tert-butyl sulfinyl propanoates 5, 6a-j, 6n and 8 .

\section{General oxidation procedure F (compounds 6k-6l and 6o)}

Following a slightly modified reported procedure, ${ }^{18 \mathrm{e}}$ in a $25 \mathrm{~mL}$ round-bottomed flask, tert-butyl 3-thiopropanoate 21 (1.0 equiv.) was dissolved in TFE ( $2 \mathrm{M}$ based on thiopropanoate) and the resulting solution was cooled to $0{ }^{\circ} \mathrm{C}$ (ice-water bath). $\mathrm{H}_{2} \mathrm{O}_{2}(30 \% \mathrm{v} / \mathrm{v}$ in water; 1.8 equiv.) was added drop-wise at the same temperature. The mixture was then allowed to warm to room temperature and stirred for 5 hours. After this time, soldium sulfite (1.8 equiv.) was added and the the mixture was vigorously stirred for 30 minutes. It was then filtered through a plug of celite, which was then washed with several portions of DCM. The filtrate was dried over $\mathrm{MgSO}_{4}$, filtered and concentrated under vacuum. The resulting crude oil was submitted to column chromatography $\left(\mathrm{SiO}_{2} ; \mathrm{DCM}: \mathrm{MeOH}\right)$ to afford desired tert-butyl 3-sulfinylpropanoates

6.

\section{Synthesis of Methyl 3-((4-methylphenyl)sulfinyl)propanoate (5)}

Following a slightly modified version of a reported procedure, ${ }^{37} 4$-methylbenzenethiol (2.8 g, $22 \mathrm{mmol}, 1.0$ equiv.), methyl acrylate $(2.0 \mathrm{~mL}, 22 \mathrm{mmol}, 1.0$ equiv.) and triethylamine $(0.30 \mathrm{~mL}$, $2.2 \mathrm{mmol}, 0.1$ equiv.) were introduced into a microwave vial equipped with a magnetic stirrer. The vial was sealed and the reaction mixture was stirred at $\mathrm{rt}$ for 10 minutes then heated to $60^{\circ} \mathrm{C}$ for 3 hours (until full conversion based on TLC analysis). The volatiles were then removed through evaporation under reduced pressure to afford methyl 3-((4-methylphenyl)thio)propanoate in 99\% yield (4.6 g, $22 \mathrm{mmol})$ with 
sufficient purity to be used in next step without further purification. Following general oxidation proce-

dure E, 5 was obtained as a colorless oil from methyl 3-((4-methylphenyl)thio)propanoate (2.50 g, 21.4 mmol) and $\mathrm{NaIO}_{4}$ (4.58 g, $\left.21.4 \mathrm{mmol}\right)$. Yield: 70\% (3.41 g, $\left.5.07 \mathrm{mmol}\right) .{ }^{1} \mathbf{H}-\mathbf{N M R}\left(400 \mathrm{MHz}, \mathrm{CDCl}_{3}\right) \delta$ $=7.52-7.50(\mathrm{~m}, 2 \mathrm{H}), 7.34(\mathrm{~d}, 2 \mathrm{H}, J=8.1 \mathrm{~Hz}), 3.67(\mathrm{~s}, 3 \mathrm{H}), 3.25-3.18(\mathrm{~m}, 1 \mathrm{H}), 3.00-2.92(\mathrm{~m}, 1 \mathrm{H}), 2.87-$ $2.79(\mathrm{~m}, 1 \mathrm{H}), 2.59-2.51(\mathrm{~m}, 1 \mathrm{H}), 2.43(\mathrm{~s}, 3 \mathrm{H}) \mathrm{ppm} .{ }^{13} \mathbf{C}\left\{{ }^{1} \mathbf{H}\right\}-\mathbf{N M R}\left(101 \mathrm{MHz}, \mathrm{CDCl}_{3}\right) \delta=171.7,141.7$ $139.7,130.0,124.1,52.1,51.2,26.0,21.4 \mathrm{ppm}$. The values of the NMR spectra are in accordance with reported literature data. ${ }^{40}$

\section{Tert-butyl 3-[(4-methylphenyl)sulfinyl]propanoate (6a)}

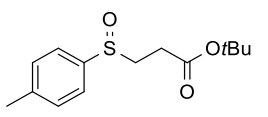

Following general procedure E, 6a was obtained as a yellow solid from 21a (1.49 g, 5.90 mmol, 1.0 equiv.) and sodium periodate (1.26 g, $5.90 \mathrm{mmol}, 1.0$ equiv.). Yield: 69\% (1.09 g, $4.06 \mathrm{mmol}) . \mathbf{~ m p : ~} 56.1-57.7^{\circ} \mathrm{C} .{ }^{1} \mathbf{H}-\mathbf{N M R}\left(400 \mathrm{MHz}, \mathrm{CDCl}_{3}\right) \delta=7.50(\mathrm{~d}, J=8.0 \mathrm{~Hz}, 2 \mathrm{H})$, $7.33(\mathrm{~d}, J=8.0 \mathrm{~Hz}, 2 \mathrm{H}), 3.14(\mathrm{ddd}, J=13.5,8.5,6.7 \mathrm{~Hz}, 1 \mathrm{H}), 2.92(\mathrm{ddd}, J=13.5,8.4,5.8 \mathrm{~Hz}, 1 \mathrm{H}) 2.73$ $(\mathrm{ddd}, J=17.2,8.5,6.8 \mathrm{~Hz}, 1 \mathrm{H}), 2.51-2.38(\mathrm{~m}, 5 \mathrm{H}), 1.42(\mathrm{~s}, 9 \mathrm{H})$ ppm. ${ }^{13} \mathbf{C}\left\{{ }^{1} \mathbf{H}\right\}-\mathbf{N M R}\left(101 \mathrm{MHz}, \mathrm{CDCl}_{3}\right)$ $\delta=170.5,141.6,139.9,130.1,124.1,81.5,51.6,28.1,27.4,21.5 \mathrm{ppm}$. The values of the NMR spectra are in accordance with reported literature data. ${ }^{8}$

\section{Tert-butyl 3-[(phenyl)sulfinyl]propanoate (6b)}

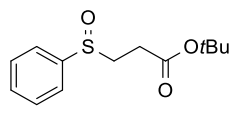

Following general procedure E, $\mathbf{6 b}$ was obtained as a colorless oil from $\mathbf{2 1 b}(1.0 \mathrm{~g}, 4.2$ mmol, 1.0 equiv.) and sodium periodate (1.1 g, $5.0 \mathrm{mmol}, 1.2$ equiv.). Yield: $56 \%(0.59$ g, $2.3 \mathrm{mmol}) .{ }^{1} \mathbf{H}-\mathbf{N M R}\left(400 \mathrm{MHz}, \mathrm{CDCl}_{3}\right) \delta=7.61(\mathrm{dt}, J=7.6,1.6 \mathrm{~Hz}, 2 \mathrm{H}), 7.58-7.47(\mathrm{~m}, 3 \mathrm{H}), 3.16$ (m, 1H), $2.92(\mathrm{ddd}, J=13.8,8.4,5.7 \mathrm{~Hz}, 1 \mathrm{H}), 2.75(\mathrm{ddd}, J=17.1,8.5,6.7 \mathrm{~Hz}, 1 \mathrm{H}), 2.43(\mathrm{ddd}, J=17.1$, 8.6, $5.8 \mathrm{~Hz}, 1 \mathrm{H}), 1.41(\mathrm{~s}, 9 \mathrm{H}) \mathrm{ppm} .{ }^{13} \mathbf{C}\left\{{ }^{1} \mathbf{H}\right\}-\mathbf{N M R}\left(101 \mathrm{MHz}, \mathrm{CDCl}_{3}\right) \delta=170.4,143.1,131.1,129.3$, 124.0, 81.6, 51.5, 28.0, $27.3 \mathrm{ppm}$. The values of the NMR spectra are in accordance with reported literature data. ${ }^{40}$

\section{Tert-butyl 3-[(4-methoxyphenyl)sulfinyl]propanoate (6c)}


Following general procedure E, 6c was obtained as a yellow solid from 21c $(0.950$

g, 3.54 mmol, 1.0 equiv.) and sodium periodate $(0.909 \mathrm{~g}, 4.25 \mathrm{mmol}, 1.2$ equiv.).

Yield: 89\% (0.900 g, $3.16 \mathrm{mmol})$. mp: $41.4-44.6{ }^{\circ} \mathrm{C} ;{ }^{1} \mathbf{H}-\mathbf{N M R}\left(400 \mathrm{MHz}, \mathrm{CDCl}_{3}\right) \delta=7.53(\mathrm{dd}, J=8.9$, $2.2 \mathrm{~Hz}, 2 \mathrm{H}), 7.00(\mathrm{dd}, J=8.9,2.2 \mathrm{~Hz}, 2 \mathrm{H}), 3.82(\mathrm{~s}, 3 \mathrm{H}), 3.08(\mathrm{ddd}, J=13.3,8.5,6.7 \mathrm{~Hz}, 1 \mathrm{H}), 2.98-2.84$ $(\mathrm{m}, 1 \mathrm{H}), 2.77-2.61(\mathrm{~m}, 1 \mathrm{H}), 2.48-2.35(\mathrm{~m}, 1 \mathrm{H}), 1.39(\mathrm{~s}, 9 \mathrm{H})$ ppm. ${ }^{13} \mathbf{C}\left\{{ }^{1} \mathbf{H}\right\}-\mathbf{N M R}\left(101 \mathrm{MHz}, \mathrm{CDCl}_{3}\right) \delta$ $=170.5,162.1,133.9,126.0,114.9,81.5,55.6,51.7,28.1,27.6$ ppm. HRMS (ESI) calcd. for $\mathrm{C}_{14} \mathrm{H}_{20} \mathrm{NaO}_{4} \mathrm{~S}^{+}[\mathrm{M}+\mathrm{Na}]^{+}$307.0974; found 307.0971. IR (film): $\widetilde{v}=3068(\mathrm{w}), 2979(\mathrm{w}), 2934$ (w), 2840 (w), $1727(\mathrm{~s}), 1596(\mathrm{~m}), 1579(\mathrm{w}), 1498(\mathrm{~m}), 1461(\mathrm{w}), 1368(\mathrm{~m}), 1304(\mathrm{~m}), 1250(\mathrm{~s}), 1155(\mathrm{~s}), 1089(\mathrm{~s})$ $1043(\mathrm{~s}), 834(\mathrm{~m}) \mathrm{cm}^{-1}$.

\section{Tert-butyl 3-[(4-bromophenyl)sulfinyl]propanoate (6d)}

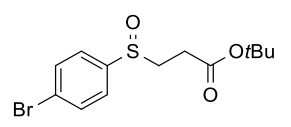

Following general procedure E, 6d was obtained as an off white solid from 21d ( $0.428 \mathrm{~g}, 1.35 \mathrm{mmol}, 1.0$ equiv.) and sodium periodate $(0.289 \mathrm{~g}, 1.35 \mathrm{mmol}, 1.0$ equiv.). Yield: 42\% (0.190 g, $0.570 \mathrm{mmol}) . \mathbf{m p :} 69.4-71.7^{\circ} \mathrm{C} .{ }^{1} \mathbf{H}-\mathbf{N M R}\left(400 \mathrm{MHz}, \mathrm{CDCl}_{3}\right) \delta=7.67(\mathrm{dd}, J=8.5$, $2.0 \mathrm{~Hz}, 2 \mathrm{H}), 7.49(\mathrm{dd}, J=8.5,2.0 \mathrm{~Hz}, 2 \mathrm{H}), 3.17(\mathrm{ddd}, J=13.5,8.2,6.9 \mathrm{~Hz}, 1 \mathrm{H}), 2.90(\mathrm{ddd}, J=13.5,8.1$, $5.8 \mathrm{~Hz}, 1 \mathrm{H}), 2.75$ (ddd, $J=17.3,8.1,6.9 \mathrm{~Hz}, 1 \mathrm{H}), 2.44$ (ddd, $J=17.3,8.2,5.8 \mathrm{~Hz}, 1 \mathrm{H}), 1.42$ (s, $9 \mathrm{H}) \mathrm{ppm}$. ${ }^{13} \mathbf{C}\left\{{ }^{1} \mathbf{H}\right\}$-NMR $\left(101 \mathrm{MHz}, \mathrm{CDCl}_{3}\right) \delta=170.4,142.4,132.6,125.8,125.7,81.8,51.6,28.1,27.3 \mathrm{ppm}$. HRMS (ESI) calcd. for $\mathrm{C}_{13} \mathrm{H}_{17}{ }^{79} \mathrm{BrNaO}_{3} \mathrm{~S}^{+}[\mathrm{M}+\mathrm{Na}]^{+}$354.9974; found 354.9985. IR (film): $\widetilde{v}=2978$ (w), $2933(\mathrm{w}), 1730(\mathrm{~s}), 1573(\mathrm{w}), 1474(\mathrm{w}), 1369(\mathrm{~m}), 1250(\mathrm{~m}), 1159(\mathrm{~s}), 1086(\mathrm{~m}), 1051(\mathrm{~s}), 1009(\mathrm{~m}), 844$ (w), $822(\mathrm{w}) \mathrm{cm}^{-1}$.

\section{Tert-butyl 3-[(4-fluorophenyl)sulfinyl]propanoate (6e)}

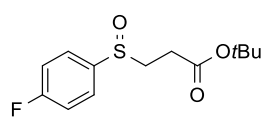

Following general procedure E, 6e was obtained as a colorless solid from 21e (0.920 g, 3.59 mmol, 1.0 equiv. $)$ and sodium periodate ( $0.921 \mathrm{~g}, 4.31 \mathrm{mmol}, 1.2$ equiv.). Yield: 83\% (0.811 g, $2.98 \mathrm{mmol}) . \mathbf{m p :} 82.4-83.5^{\circ} \mathrm{C} .{ }^{\mathbf{1}} \mathbf{H}-\mathrm{NMR}\left(400 \mathrm{MHz}, \mathrm{CDCl}_{3}\right) \delta=7.61(\mathrm{ddd}, J=9.8,5.0$ $2.5 \mathrm{~Hz}, 2 \mathrm{H}), 7.22(\mathrm{~m}, 2 \mathrm{H}), 3.14(\mathrm{ddd}, J=13.4,8.3,6.9 \mathrm{~Hz}, 1 \mathrm{H}), 2.91(\mathrm{ddd}, J=13.5,8.1,5.8 \mathrm{~Hz}, 1 \mathrm{H})$, 
$2.74(\mathrm{ddd}, J=17.3,8.1,7.0 \mathrm{~Hz}, 1 \mathrm{H}), 2.45(\mathrm{ddd}, J=17.3,8.3,5.8 \mathrm{~Hz}, 1 \mathrm{H}), 1.41(\mathrm{~s}, 9 \mathrm{H}) \mathrm{ppm} .{ }^{\mathbf{1 3}} \mathbf{C}\left\{{ }^{1} \mathbf{H}\right\}-$ NMR $\left(101 \mathrm{MHz}, \mathrm{CDCl}_{3}\right) \delta=170.3,164.4(\mathrm{~d}, J=251.5 \mathrm{~Hz}), 138.6(\mathrm{~d}, J=3.1 \mathrm{~Hz}), 126.3(\mathrm{~d}, J=9.0 \mathrm{~Hz})$, $116.7(\mathrm{~d}, J=22.7 \mathrm{~Hz}), 81.7,51.7,28.0,27.3 \mathrm{ppm} .{ }^{19} \mathbf{F}$ NMR $\left(376 \mathrm{MHz}, \mathrm{CDCl}_{3}\right) \delta=-108.5 \mathrm{ppm} . \mathbf{H R M S}$ calcd. for $\mathrm{C}_{13} \mathrm{H}_{17} \mathrm{FNaO}_{3} \mathrm{~S}^{+}[\mathrm{M}+\mathrm{Na}]^{+}$295.0775; found 295.0777. IR (film): $\widetilde{v}=3062$ (w), 2977 (w), 2931 (w), $1728(\mathrm{~s}), 1591(\mathrm{~m}), 1493(\mathrm{~s}), 1369(\mathrm{~m}), 1295(\mathrm{w}), 1233(\mathrm{~s}), 1153(\mathrm{~s}), 1086(\mathrm{~m}), 838(\mathrm{~s}), 815(\mathrm{~m}) \mathrm{cm}^{-}$ 1 .

\section{Tert-butyl 3-[(4-trifluoromethylphenyl)sulfinyl]propanoate (6f)}

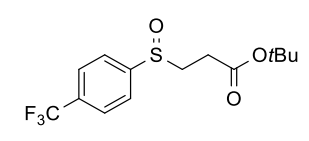

Following general procedure E, $6 \mathbf{f}$ was obtained as an off-white solid from $21 \mathbf{f}(0.645$ g, $2.10 \mathrm{mmol}, 1.0$ equiv.) and sodium periodate (0.540 g, $2.53 \mathrm{mmol}, 1.2$ equiv.). Yield: 87\% (0.592 g, $1.84 \mathrm{mmol}) . \mathbf{m p :} 64.0-64.8{ }^{\circ} \mathrm{C} .{ }^{1} \mathbf{H}-\mathbf{N M R}\left(400 \mathrm{MHz}, \mathrm{CDCl}_{3}\right) \delta=7.94-7.49(\mathrm{~m}$, 4H), $3.23(\mathrm{ddd}, J=13.6,8.0,7.1 \mathrm{~Hz}, 1 \mathrm{H}), 2.93(\mathrm{ddd}, J=13.6,7.8,5.8 \mathrm{~Hz}, 1 \mathrm{H}), 2.78(\mathrm{ddd}, J=17.4,7.8$, $7.1 \mathrm{~Hz}, 1 \mathrm{H}), 2.46(\mathrm{ddd}, J=17.4,8.0,5.8 \mathrm{~Hz}, 1 \mathrm{H}), 1.41(\mathrm{~s}, 9 \mathrm{H})$ ppm. ${ }^{13} \mathbf{C}\left\{{ }^{1} \mathbf{H}\right\}-\mathbf{N M R}\left(101 \mathrm{MHz}, \mathrm{CDCl}_{3}\right)$ $\delta=170.1,147.7,133.1(\mathrm{q}, J=32.8 \mathrm{~Hz}), 126.3(\mathrm{q}, J=3.8 \mathrm{~Hz}), 124.6,123.5(\mathrm{q}, J=272.6 \mathrm{~Hz}), 81.8,51.4$, 28.0, 27.0 ppm. ${ }^{19} \mathbf{F}$ NMR (376 $\left.\mathrm{MHz}, \mathrm{CDCl}_{3}\right) \delta=-62.9$ ppm. HRMS calcd. for $\mathrm{C}_{14} \mathrm{H}_{17} \mathrm{~F}_{3} \mathrm{NaO}_{3} \mathrm{~S}^{+}$ $[\mathrm{M}+\mathrm{Na}]^{+}$345.0743; found 345.0737. IR (film): $\widetilde{v}=3053(\mathrm{w}), 2988(\mathrm{w}), 1728(\mathrm{~m}), 1422(\mathrm{w}), 1403(\mathrm{w})$, 1368 (w), 1325 (m), 1265 (s), 1169 (m), 1133 (m), 1060 (m), $1016(\mathrm{w}), 845(\mathrm{w}) \mathrm{cm}^{-1}$.

\section{Tert-butyl 3-[(4-nitrophenyl)sulfinyl]propanoate (6g)}

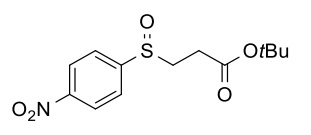

Following general procedure $\mathbf{E}, \mathbf{6 g}$ was obtained as a yellow crystalline solid from $21 \mathrm{~g}(0.400 \mathrm{~g}, 1.41 \mathrm{mmol}, 1.0$ equiv. $)$ and sodium periodate $(0.362 \mathrm{~g}, 1.69 \mathrm{mmol}, 1.2$ equiv.). Yield: $29 \%$ (0.124 g, $0.414 \mathrm{mmol}) . \mathbf{m p}: 90.4-91.2{ }^{\circ} \mathrm{C} .{ }^{1} \mathbf{H}-\mathbf{N M R}\left(400 \mathrm{MHz}, \mathrm{CDCl}_{3}\right) \delta=8.38(\mathrm{~m}$, 2H), $7.82(\mathrm{~m}, 2 \mathrm{H}), 3.26(\mathrm{dt}, J=13.5,7.6 \mathrm{~Hz}, 1 \mathrm{H}), 2.95(\mathrm{ddd}, J=13.4,7.6,5.7 \mathrm{~Hz}, 1 \mathrm{H}), 2.81(\mathrm{dt}, J=17.5$, $7.5 \mathrm{~Hz}, 1 \mathrm{H}), 2.48(\mathrm{ddd}, J=17.5,7.8,5.7 \mathrm{~Hz}, 1 \mathrm{H}), 1.41(\mathrm{~s}, 9 \mathrm{H}) \mathrm{ppm} .{ }^{13} \mathbf{C}\left\{{ }^{1} \mathbf{H}\right\}-\mathbf{N M R}\left(101 \mathrm{MHz}, \mathrm{CDCl}_{3}\right)$ $\delta=170.0,150.9,149.6,125.2,124.4,82.0,51.6,28.0,27.0$ ppm. HRMS (ESI) calcd. for $\mathrm{C}_{13} \mathrm{H}_{17} \mathrm{NNaO}_{5} \mathrm{~S}^{+}$ 
$[\mathrm{M}+\mathrm{Na}]^{+}$322.0720; found 322.0715. IR (film): $\widetilde{v}=3121(\mathrm{w}), 2966(\mathrm{~m}), 2922(\mathrm{~m}), 2854(\mathrm{~m}), 1727$ (s), 1522 (s), 1344 (s), 1258 (m), 1164 (s), 1083 (m), 1044 (s), 854 (s) cm .

\section{Tert-butyl 3-[(2-bromophenyl)sulfinyl]propanoate (6h)}

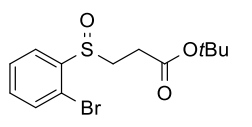

Following general procedure $\mathbf{E}, \mathbf{6 h}$ was obtained as a colorless crystalline solid from $21 \mathrm{~h}(0.734 \mathrm{~g}, 2.32 \mathrm{mmol}, 1.0$ equiv. $)$ and sodium periodate $(0.500 \mathrm{~g}, 2.33 \mathrm{mmol}, 1.0$ equiv.). Yield: $79 \%$ (0.609 g, $1.83 \mathrm{mmol})$. mp: $72.6-73.5{ }^{\circ} \mathrm{C} .{ }^{\mathbf{1}} \mathbf{H}-\mathbf{N M R}\left(400 \mathrm{MHz}, \mathrm{CDCl}_{3}\right) \delta=7,83(\mathrm{~m}$, 1H), $7.66-7.49(\mathrm{~m}, 2 \mathrm{H}), 7.37(\mathrm{td}, J=7.6,1.7 \mathrm{~Hz}, 1 \mathrm{H}), 3.39(\mathrm{ddd}, J=13.7,8.9,6.5 \mathrm{~Hz}, 1 \mathrm{H}), 3.08$ (ddd, $J=13.7,8.9,5.8 \mathrm{~Hz}, 1 \mathrm{H}), 2.79(\mathrm{ddd}, J=17.1,8.9,6.5 \mathrm{~Hz}, 1 \mathrm{H}), 2.44(\mathrm{ddd}, J=17.1,8.9,5.8 \mathrm{~Hz}, 1 \mathrm{H})$, $1.40(\mathrm{~s}, 9 \mathrm{H}) \mathrm{ppm} .{ }^{13} \mathbf{C}\{\mathbf{1 H}\}-\mathbf{N M R}\left(101 \mathrm{MHz}, \mathrm{CDCl}_{3}\right) \delta=170.3,142.5,133.1,132.4,128.4,126.8,118.9$, 81.5, 47.8, 28.0, 26.8 ppm. HRMS calcd. for $\mathrm{C}_{13} \mathrm{H}_{17}{ }^{79} \mathrm{BrNaO}_{3} \mathrm{~S}^{+}[\mathrm{M}+\mathrm{Na}]^{+}$354.9974; found 354.9976. IR (film): $\widetilde{v}=3062$ (w), 2979 (w), 2930 (w), 2854 (w), 1730 (s), 1568 (w), 1449 (m), 1423 (w), 1393 (w), $1368(\mathrm{~m}), 1245$ (m), 1155 (s), 1095 (m), 1062 (s), 1016 (s), $954(\mathrm{w}), 843$ (m) cm².

\section{Tert-butyl 3-[(3-methylphenyl)sulfinyl]propanoate (6i)}

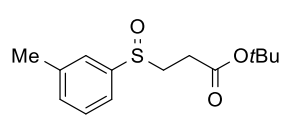

Following general procedure E, $\mathbf{6 i}$ was obtained as a pale yellow oil from $21 \mathbf{i}(1.50$ g, 5.94 mmol, 1.0 equiv.) and sodium periodate (1.27 g, 5.94 mmol, 1.0 equiv.). Yield: 66\% (1.05 g, $3.91 \mathrm{mmol}) .{ }^{1} \mathbf{H}-\mathbf{N M R}\left(400 \mathrm{MHz}, \mathrm{CDCl}_{3}\right) \delta=7.42(\mathrm{~m}, J=1.8,0.9 \mathrm{~Hz}, 1 \mathrm{H}), 7.39-7.34(\mathrm{~m}$, 2H), $7.27(\mathrm{~m}, 1 \mathrm{H}), 3.13(\mathrm{ddd}, J=13.4,8.6,6.7 \mathrm{~Hz}, 1 \mathrm{H}), 2.90(\mathrm{ddd}, J=13.4,8.6,5.7 \mathrm{~Hz}, 1 \mathrm{H}), 2.73$ (ddd, $J=17.1,8.6,6.7 \mathrm{~Hz}, 1 \mathrm{H}), 2.57-2.35(\mathrm{~m}, 4 \mathrm{H}), 1.40(\mathrm{~s}, 9 \mathrm{H})$ ppm. ${ }^{13} \mathbf{C}\left\{{ }^{1} \mathbf{H}\right\}-\mathbf{N M R}\left(101 \mathrm{MHz}, \mathrm{CDCl}_{3}\right) \delta=$ 170.4, 143.0, 139.5, 131.9, 129.1, 124.3, 121.1, 81.5, 51.5, 28.0, 27.4, 21.4 ppm. HRMS (APPI) calcd. for $\mathrm{C}_{14} \mathrm{H}_{20} \mathrm{O}_{2} \mathrm{~S}^{+\circ}[\mathrm{M}]^{+\circ}$ 252.1179; found 252.1174. IR (film): $\widetilde{v}=2984$ (w), 2928 (w), $1728(\mathrm{~s}), 1362$ (m), 1238 (m), 1153 (s), 1081 (m), 1045 (s), 974 (w), 948 (w), 842 (m), 785 (m), 693 (m) cm².

\section{Tert-butyl 3-[(3-bromophenyl)sulfinyl]propanoate (6j)}

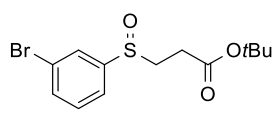

Following general procedure E, $\mathbf{6 j}$ was obtained as a colorless oil from $\mathbf{2 1 \mathbf { j }}(0.815 \mathrm{~g}$, $2.57 \mathrm{mmol}, 1.0$ equiv.) and sodium periodate (0.659 g, $3.08 \mathrm{mmol}, 1.2$ equiv.). Yield: 
88\% (0.754 g, $2.26 \mathrm{mmol}) .{ }^{1} \mathbf{H}-\mathbf{N M R}\left(400 \mathrm{MHz}, \mathrm{CDCl}_{3}\right) \delta=7.77(\mathrm{t}, J=1.8 \mathrm{~Hz}, 1 \mathrm{H}), 7.61$ (ddd, $J=7.9$, 1.9, $1.0 \mathrm{~Hz}, 1 \mathrm{H}), 7.51(\mathrm{ddd}, J=7.9,1.8,1.0 \mathrm{~Hz}, 1 \mathrm{H}), 7.39(\mathrm{t}, J=7.9 \mathrm{~Hz}, 1 \mathrm{H}), 3.18(\mathrm{ddd}, J=13.5,8.2$, $7.0 \mathrm{~Hz}, 1 \mathrm{H}), 2.92(\mathrm{ddd}, J=13.5,8.2,5.8 \mathrm{~Hz}, 1 \mathrm{H}), 2.76(\mathrm{ddd}, J=17.3,8.2,7.0 \mathrm{~Hz}, 1 \mathrm{H}), 2.45(\mathrm{ddd}, J=$ 17.3, 8.2, $5.8 \mathrm{~Hz}, 1 \mathrm{H}), 1.42(\mathrm{~s}, 9 \mathrm{H}) \mathrm{ppm} .{ }^{13} \mathbf{C}\left\{{ }^{1} \mathbf{H}\right\}$-NMR $\left(101 \mathrm{MHz}, \mathrm{CDCl}_{3}\right) \delta=170.2,145.5,134.2$, 130.8, 126.9, 123.6, 122.6, 81.7, 51.6, 28.027 .2 ppm. HRMS (ESI) calcd. for $\mathrm{C}_{13} \mathrm{H}_{17}{ }^{79} \mathrm{BrNaO}_{3} \mathrm{~S}^{+}$ $[\mathrm{M}+\mathrm{Na}]^{+}$354.9974; found 354.9968. IR (film): $\widetilde{v}=3056(\mathrm{w}), 2980(\mathrm{~m}), 1728(\mathrm{~s}), 1569(\mathrm{w}), 1491(\mathrm{w})$, 1459 (w), 1416 (w), 1395 (w), 1368 (m), 1248 (m), 1158 (s), $1079(\mathrm{w}), 1050(\mathrm{~m}), 844$ (w) cm ${ }^{-1}$.

\section{Tert-butyl 3-[(pyridin-2-yl)sulfinyl]propanoate (6k)}

Following general procedure F, 6k was obtained as a yellow oil from $21 \mathbf{k}(0.500 \mathrm{~g}, 2.09$ mmol, 1.0 equiv.), $\mathrm{H}_{2} \mathrm{O}_{2}(30 \% \mathrm{v} / \mathrm{v}$ in water; $0.39 \mathrm{~mL}, 3.8 \mathrm{mmol}, 1.8$ equiv.) and sodium sulfite $(0.474 \mathrm{~g}$, 3.76 mmol, 1.8 equiv.). Yield: $35 \%$ (0.474 g, $1.29 \mathrm{mmol}) .{ }^{1} \mathbf{H}-\mathbf{N M R}\left(400 \mathrm{MHz}, \mathrm{CDCl}_{3}\right) \delta=8.63(\mathrm{ddd}, J$ $=4.7,1.7,1.0 \mathrm{~Hz}, 1 \mathrm{H}), 7.96(\mathrm{~m}, 1 \mathrm{H}), 7.92(\mathrm{~m}, 1), 7.37$ (ddd, $J=7.1,4.7,1.6 \mathrm{~Hz}, 1 \mathrm{H}), 3.44(\mathrm{ddd}, J=13.6$, 9.6, $6.0 \mathrm{~Hz}, 1 \mathrm{H}), 3.13(\mathrm{ddd}, J=13.6,9.5,5.8 \mathrm{~Hz}, 1 \mathrm{H}), 2.79$ (ddd, $J=17.0,9.4,6.0 \mathrm{~Hz}, 1 \mathrm{H}), 2.34$ (ddd, $J$ $=17.0,9.5,5.7 \mathrm{~Hz}, 1 \mathrm{H}), 1.41(\mathrm{~s}, 9 \mathrm{H}) \mathrm{ppm} .{ }^{13} \mathbf{C}\left\{{ }^{1} \mathbf{H}\right\}-\mathbf{N M R}\left(101 \mathrm{MHz}, \mathrm{CDCl}_{3}\right) \delta=170.3,163.9,149.8$, 137.9, 124.6, 120.1, 81.4, 48.3, 28.0, 26.8 ppm. HRMS (ESI) calcd. for $\mathrm{C}_{12} \mathrm{H}_{17} \mathrm{NNaO}_{3} \mathrm{~S}^{+}[\mathrm{M}+\mathrm{Na}]^{+}$ 278.0821; found 278.0825. IR (film): $\widetilde{v}=2977(\mathrm{~m}), 2941$ (w), $3054(\mathrm{w}), 1730$ (s), $1576(\mathrm{~m}), 1458$ (m), 1423 (m), 1368 (m), 1248 (s), 1154 (s), 1053 (s), 849 (w) $\mathrm{cm}^{-1}$.

\section{Tert-butyl 3-((thiophen-2-yl)sulfinyl)propanoate (6l)}

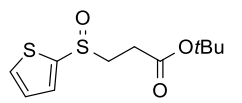

Following general procedure F, 61 was obtained as a yellow oil from $211(0.900 \mathrm{~g}, 3.68$ mmol, 1.0 equiv.), $\mathrm{H}_{2} \mathrm{O}_{2}(30 \% \mathrm{v} / \mathrm{v}$ in water; $0.68 \mathrm{~mL}, 6.6 \mathrm{mmol}, 1.8$ equiv.) and sodium sulfite (0.836 g, 6.63 mmol, 1.8 equiv.). Yield: $35 \%$ (0.335 g, 1.29 mmol). mp: $38.2-39.0{ }^{\circ} \mathrm{C} .{ }^{1} \mathbf{H}-\mathbf{N M R}$ $\left(400 \mathrm{MHz}, \mathrm{CDCl}_{3}\right) \delta=7.66(\mathrm{dd}, J=5.0,1.3 \mathrm{~Hz}, 1 \mathrm{H}), 7.47(\mathrm{dd}, J=3.7,1.3 \mathrm{~Hz}, 1 \mathrm{H}), 7.14(\mathrm{dd}, J=5.0$, $3.7 \mathrm{~Hz}, 1 \mathrm{H}), 3.25(\mathrm{dd}, J=7.6,7.0 \mathrm{~Hz}, 2 \mathrm{H}), 2.74(\mathrm{~m}, 1 \mathrm{H}), 2.58(\mathrm{dt}, J=17.2,7.2 \mathrm{~Hz}, 1 \mathrm{H}), 1.44(\mathrm{~s}, 9 \mathrm{H})$ ppm. ${ }^{13} \mathbf{C}\left\{{ }^{1} \mathbf{H}\right\}-N M R\left(101 \mathrm{MHz}, \mathrm{CDCl}_{3}\right) \delta=170.1,145.3,131.2,129.7,127.5,81.7,53.0,28.2,28.1 \mathrm{ppm}$. 
HRMS (ESI) calcd. for $\mathrm{C}_{11} \mathrm{H}_{16} \mathrm{NaO}_{3} \mathrm{~S}_{2}{ }^{+}[\mathrm{M}+\mathrm{Na}]^{+}$283.0433; found 283.0440. IR (film): $\widetilde{v}=3084$ (w), $2982(\mathrm{w}), 2906(\mathrm{w}), 2856(\mathrm{w}), 1726(\mathrm{~s}), 1402(\mathrm{w}), 1364(\mathrm{~m}), 1250(\mathrm{~m}), 1161(\mathrm{~s}), 1047(\mathrm{~s}), 850(\mathrm{~m}) \mathrm{cm}^{-1}$.

\section{Tert-butyl 3-((2-methylfuran-3-yl)sulfinyl)propanoate (6m)}

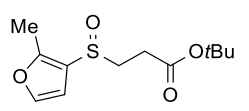

Following general procedure $\mathbf{F}, \mathbf{6 m}$ was obtained as a yellow oil from $\mathbf{2 1} \mathbf{m}(0.828 \mathrm{~g}$,

3.42 mmol, 1.0 equiv.) and $\mathrm{H}_{2} \mathrm{O}_{2}(30 \% \mathrm{v} / \mathrm{v}$ in water; $0.64 \mathrm{~mL}, 6.2 \mathrm{mmol}, 1.8$ equiv. $)$, sodium sulfite (0.775 g, 6.15 mmol, 1.8 equiv.). Yield: 89\% (0.784 g, 3.03 mmol). ${ }^{1} \mathbf{H}-\mathbf{N M R}(400 \mathrm{MHz}$, $\left.\mathrm{CDCl}_{3}\right) \delta=7.39(\mathrm{~d}, J=2.1 \mathrm{~Hz}, 1 \mathrm{H}), 6.66(\mathrm{~d}, J=2.1 \mathrm{~Hz}, 1 \mathrm{H}), 3.25(\mathrm{ddd}, J=13.1,7.8,6.5 \mathrm{~Hz}, 1 \mathrm{H}), 3.08$ $(\mathrm{ddd}, J=13.1,8.0,6.9 \mathrm{~Hz}, 1 \mathrm{H}), 2.82-2.50(\mathrm{~m}, 2 \mathrm{H}), 2.44(\mathrm{~s}, 3 \mathrm{H}), 1.44(\mathrm{~s}, 9 \mathrm{H}) \mathrm{ppm} .{ }^{13} \mathbf{C}\left\{{ }^{1} \mathbf{H}\right\}-\mathbf{N M R}(101$ $\mathrm{MHz}_{\mathrm{CDCl}} \mathrm{CD} \delta=170.1,154.8,142.7,121.8,106.6,81.6,49.8,28.8,28.0,12.4$ ppm. HRMS (ESI) calcd. for $\mathrm{C}_{12} \mathrm{H}_{18} \mathrm{NaO}_{4} \mathrm{~S}^{+}[\mathrm{M}+\mathrm{Na}]^{+} 281.0818$; found 281.0820. IR (film): $\widetilde{v}=2942(\mathrm{w}), 2861(\mathrm{w}), 1592(\mathrm{w})$, $1523(\mathrm{w}), 1466(\mathrm{w}), 1385(\mathrm{w}), 1227(\mathrm{w}), 1070(\mathrm{~s}), 882(\mathrm{~m}), 765(\mathrm{~s}), 680(\mathrm{~m}), 655(\mathrm{~m}) \mathrm{cm}^{-1}$.

\section{Tert-butyl 3-(pentylsulfinyl)propanoate (6n)}

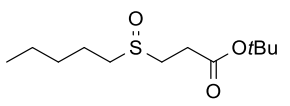

Following general procedure E, 6c was obtained as a yellow oil from 1d (0.699 g, $3.01 \mathrm{mmol}, 1.0$ equiv.) and sodium periodate (0.643 g, $3.01 \mathrm{mmol}, 1.0$ equiv.). Yield: 65\% (0.486 g, $1.96 \mathrm{mmol}) .{ }^{1} \mathbf{H}-\mathbf{N M R}\left(400 \mathrm{MHz}, \mathrm{CDCl}_{3}\right) \delta=2.99(\mathrm{ddd}, J=12.5,8.1,7.3 \mathrm{~Hz}, 1 \mathrm{H}), 2.82$ (m, 1H), 2.79-2.6 (m, 4H), 1.84-1.71 (m, 2H), $1.46(\mathrm{~s}, 9 \mathrm{H}), 1.46-1.34(\mathrm{~m}, 4 \mathrm{H}), 0.91(\mathrm{t}, J=7.1 \mathrm{~Hz}, 3 \mathrm{H})$ ppm. ${ }^{13} \mathbf{C}\left\{{ }^{1} \mathbf{H}\right\}$-NMR $\left(101 \mathrm{MHz}, \mathrm{CDCl}_{3}\right) \delta=170.7,81.7,52.8,47.2,31.1,28.3,28.2,22.5,22.4,14.0$ ppm. HRMS (ESI) calcd. for $\mathrm{C}_{12} \mathrm{H}_{24} \mathrm{O}_{3} \mathrm{NaS}^{+}[\mathrm{M}+\mathrm{Na}]^{+}$271.1344; found 271.1345. IR (film): $\widetilde{v}=2960$ (w), 2930 (m), 2869 (w), 1727 (s), 1462 (w), $1419(\mathrm{w}), 1393$ (w), $1366(\mathrm{~m}), 1297$ (w), 1247 (m), 1154 (s), $1035(\mathrm{~s}), 977(\mathrm{w}), 951(\mathrm{w}), 845(\mathrm{w}) \mathrm{cm}^{-1}$.

\section{Tert-butyl 3-(cyclohexylsulfinyl)propanoate (6o)}

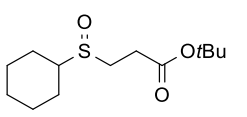

Following general procedure F, 60 was obtained as a yellow oil from $210(0.800 \mathrm{~g}, 3.27$ mmol, 1.0 equiv.) and $\mathrm{H}_{2} \mathrm{O}_{2}(30 \% \mathrm{v} / \mathrm{v}$ in water; $0.60 \mathrm{~mL}, 5.9 \mathrm{mmol}, 1.8$ equiv. $)$, sodium sulfite (0.743 g, 5.89 mmol, 1.8 equiv.). Yield: $62 \%$ (0.530 g, 1.96 mmol). ${ }^{1} \mathbf{H}-\mathbf{N M R}\left(400 \mathrm{MHz}, \mathrm{CDCl}_{3}\right)$ 
$\delta=2.93(\mathrm{~m}, 1 \mathrm{H}), 2.83-2.69(\mathrm{~m}, 3 \mathrm{H}), 2.53(\mathrm{tt}, J=11.5,3.6 \mathrm{~Hz}, 1 \mathrm{H}), 2.09(\mathrm{~m}, 1 \mathrm{H}), 1.95-1.78(\mathrm{~m}, 3 \mathrm{H})$, $1.68(\mathrm{~m}, 1 \mathrm{H}), 1.53-1.20(\mathrm{~m}, 14 \mathrm{H}) \mathrm{ppm} .{ }^{13} \mathbf{C}\left\{{ }^{1} \mathbf{H}\right\}-\mathbf{N M R}\left(101 \mathrm{MHz}, \mathrm{CDCl}_{3}\right) \delta=170.7,81.5,59.3,43.9$ 28.4, 28.0, 26.2, 25.5, 25.4, 25.1, 25.0 ppm. HRMS (ESI) calcd. for $\mathrm{C}_{13} \mathrm{H}_{24} \mathrm{NaO}_{3} \mathrm{~S}^{+}[\mathrm{M}+\mathrm{Na}]^{+}$283.1338; found 283.1344. IR (film): $\widetilde{v}=2982(\mathrm{~m}), 2932$ (m), $2849(\mathrm{~m}), 1726(\mathrm{~s}), 1453(\mathrm{~m}), 1364(\mathrm{~m}), 1237$ (m), $1155(\mathrm{~s}), 1034(\mathrm{~s}), 850(\mathrm{~m}) \mathrm{cm}^{-1}$.

\section{Synthesis of 1-Methyl-4-[(2-(phenylsulfonyl)ethyl)sulfinyl]benzene (7)}

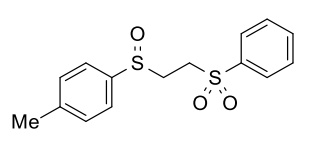

Following a reported procedure, ${ }^{18 \mathrm{c}} 4$-methylbenzenethiol $(1.0 \mathrm{~g}, 8.1 \mathrm{mmol}, 1$ equiv.), potassium carbonate $(0.111 \mathrm{~g}, 0.805 \mathrm{mmol}, 0.1$ equiv. $)$ and dichloromethane $(4.0 \mathrm{~mL})$ were introduced into a $25 \mathrm{~mL}$ one-necked flask equipped with a magnetic stirrer. Vinylsulfonyl benzene ( $1.35 \mathrm{~g}, 8.05 \mathrm{mmol}, 1.0$ equiv.) was then added portion wise. The mixture was stirred at room temperature for 24 hours. It was then diluted with dichloromethane $(5 \mathrm{~mL})$, washed with aqueous $\mathrm{NaOH}(1.0 \mathrm{M} ; 5$ $\mathrm{mL})$, brine $(2 \times 5 \mathrm{~mL})$, dried over $\mathrm{MgSO}_{4}$ and filtered. The crude product was concentrated under reduced pressure affording (2-(phenylsulfonyl)ethyl)(p-tolyl)sulfane as a colorless solid. The compound was used directly in next stage with no further purification. Following a slightly modified version of a reported procedure,${ }^{16}$ in a two-necked flask equipped with a magnetic stirrer, (2-(phenylsulfonyl)ethyl)( $p$-tolyl)sulfane $(2.35 \mathrm{~g}, 8.04 \mathrm{mmol}, 1.0$ equiv. $)$ was dissolved in methanol $(62 \mathrm{~mL})$ and cooled to $0{ }^{\circ} \mathrm{C}$. Sodium periodate (1.72 g, $8.04 \mathrm{mmol}, 1.0$ equiv.) dissolved in water (20 mL) was then added dropwise using a syringe over a period of 30 minutes. The reaction mixture was then left to warm to room temperature under stirring overnight. The precipitated solids were then filtered off through a Celite pad and washed with methanol $(20 \mathrm{~mL})$. Methanol was evaporated under reduced pressure. The obtained aqueous phase was extracted three times with DCM $(3 \times 20 \mathrm{~mL})$. The organic phases were combined washed with brine $(10 \mathrm{~mL})$, dried over $\mathrm{Na}_{2} \mathrm{SO}_{4}$ and filtered. Solvents were then evaporated under reduced pressure before submitting the crude to column chromatography (silica gel, DCM/EtOAc, 24/1 to 4/1) affording pure 7 as a yellow solid in 26\% yield (0.635 g, $2.06 \mathrm{mmol}) .{ }^{\mathbf{1}} \mathbf{H}-\mathbf{N M R}\left(400 \mathrm{MHz}, \mathrm{CDCl}_{3}\right) \delta=7.83-7.88(\mathrm{~m}, 2 \mathrm{H})$, $7.68(\mathrm{tt}, J=7.5,1.3 \mathrm{~Hz}, 1 \mathrm{H}), 7.54-7.60(\mathrm{~m}, 2 \mathrm{H}), 7.39-7.44(\mathrm{~m}, 2 \mathrm{H}), 7.31-7.36(\mathrm{~m}, 2 \mathrm{H}), 3.53(\mathrm{ddd}, J=$ 
13.7, 12.1, $4.0 \mathrm{~Hz}, 1 \mathrm{H}), 3.30$ (ddd, $J=13.0,12.0,4.0 \mathrm{~Hz}, 1 \mathrm{H}), 3.06$ (ddd, $J=13.7,12.0,4.5 \mathrm{~Hz}, 1 \mathrm{H}), 2.95$

(ddd, $J=13.0,12.0,4.5 \mathrm{~Hz}, 1 \mathrm{H}), 2.42(\mathrm{~s}, 3 \mathrm{H}) \mathrm{ppm} .{ }^{13} \mathbf{C}\left\{{ }^{1} \mathbf{H}\right\}-\mathbf{N M R}\left(101 \mathrm{MHz}, \mathrm{CDCl}_{3}\right) \delta=142.2,138.5$, $134.3,130.3,128.1,123.9,48.2,47.8,21.5 \mathrm{ppm}$. Two carbons are not resolved. The values of the NMR spectra are in accordance with reported literature data. ${ }^{18 \mathrm{e}}$

\section{1-Methyl-4-[(2-nitroethyl)sulfinyl]benzene (8)}

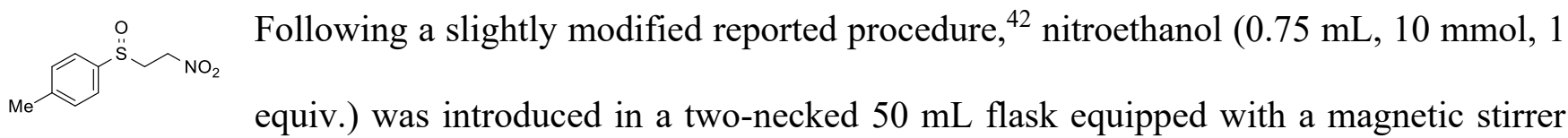
and put in the dark. Anhydrous dichloromethane $(16 \mathrm{~mL})$, pyridine $(0.85 \mathrm{~mL}, 10 \mathrm{mmol}, 1$ equiv. $)$ and acetic anhydride (1.1 mL, $12 \mathrm{mmol}, 1.1$ equiv.) were then added. and the reaction mixture was stirred for 5 hours under inert atmosphere. The reaction mixture was then poured onto aqueous $\mathrm{HCl}(1.0 \mathrm{M} ; 10 \mathrm{~mL})$. The aqueous layer was extracted with diethyl ether $(2 \times 15 \mathrm{~mL})$. The organic layers were combined, washed with aqueous $\mathrm{HCl}(1.0 \mathrm{M} ; 10 \mathrm{~mL})$, water $(3 \times 10 \mathrm{~mL})$ and brine $(10 \mathrm{~mL})$, dried over $\mathrm{MgSO}_{4}$ and filtered. The solvents were evaporated under reduced pressure yielding 2-nitroethylacetate (1.02 g, 7.69 mmol, 74\% yield) as a crude yellow oil. The compound was used directly in next step with no further purification. In a two-necked $50 \mathrm{~mL}$ flask equipped with a magnetic stirrer, 2-nitroethyl acetate $(1.02 \mathrm{~g}$, 7.69 mmol, 1 equiv. $)$ was stirred in acetonitrile $(15 \mathrm{~mL})$ and cooled to $-10{ }^{\circ} \mathrm{C}$ with an ice-salt bath. 4 Methylbezenethiol ( $0.955 \mathrm{~g}, 7.69 \mathrm{mmol}, 1$ equiv.) was added in one portion and the reaction mixture was stirred at $-10{ }^{\circ} \mathrm{C}$ for 5 minutes. A solution of triethylamine (1.3 $\mathrm{mL}, 9.3 \mathrm{mmol}, 1.2$ equiv.) in acetonitrile $(6 \mathrm{~mL})$ was added dropwise over a period of 20 minutes. The temperature was left to rise slowly to $0{ }^{\circ} \mathrm{C}$ over a period of 2 hours. The reaction mixture was poured onto aqueous $\mathrm{HCl}(1.0 \mathrm{M} ; 10 \mathrm{~mL})$. Acetonitrile was then evaporated under reduced pressure. The obtained aqueous solution was extracted with pentane ( $3 \times 15 \mathrm{~mL})$. The organic layers were combined, washed with brine $(10 \mathrm{~mL})$, dried over $\mathrm{MgSO}_{4}$, filtered and concentrated under reduced pressure to afford (2-nitroethyl)( $p$-tolyl)sulfane (1.4 g, $7.1 \mathrm{mmol}, 92 \%$ yield) as a yellow oil, which was used in next step with no further purification. Following the general procedure $\mathbf{E}, \mathbf{8}$ was obtained as a yellow oil from (2-nitroethyl)(p-tolyl)sulfane (1.38 g, $7.00 \mathrm{mmol})$ and 
sodium periodate (1.50 g, $7.00 \mathrm{mmol})$. Yield: 51\% (0.757 g, $3.55 \mathrm{mmol}) .{ }^{1} \mathbf{H}-\mathbf{N M R}\left(400 \mathrm{MHz}, \mathrm{CDCl}_{3}\right) \delta$ $=7.50(\mathrm{~d}, J=8.2 \mathrm{~Hz}, 2 \mathrm{H}), 7.35(\mathrm{~d}, J=8.2 \mathrm{~Hz}, 2 \mathrm{H}), 4.83(\mathrm{dt}, J=15.0,7.2 \mathrm{~Hz}, 1 \mathrm{H}), 4.51(\mathrm{ddd}, J=15.0$, 7.2, $5.9 \mathrm{~Hz}, 1 \mathrm{H}), 3.57(\mathrm{dt}, J=14.2,7.2 \mathrm{~Hz}, 1 \mathrm{H}), 3.21(\mathrm{ddd}, J=14.2,7.2,5.8 \mathrm{~Hz}, 1 \mathrm{H}), 2.42(\mathrm{~s}, 3 \mathrm{H}) \mathrm{ppm}$. ${ }^{13} \mathbf{C}\left\{{ }^{1} \mathbf{H}\right\}$-NMR $\left(101 \mathrm{MHz}, \mathrm{CDCl}_{3}\right) \delta=142.6,138.6,130.5,123.9,67.3,51.8,21.6$ ppm. The values of the NMR spectra are in accordance with reported literature data. ${ }^{18 \mathrm{c}}$

\section{Alkynylation of sulfoxides}

\section{General procedure G: Synthesis of (hetero)aryl alkynyl sulfoxides (10a-10n, 10p and 10q)}

The tert-butyl 3-(sulfinyl) propanoate (6a-6n) (0.56 mmol, 1.11 equiv.) and $\mathrm{R}^{2}$-EBX (9a-9c) (0.5 mmol, 1 equiv.) were introduced into an oven-dried two-neck $25 \mathrm{~mL}$ flask equipped with a magnetic stirrer. The reaction vessel was evacuated and backfilled with nitrogen (3 times). The reaction vessel was transferred into a glove-box where potassium tert-butoxide ( $0.59 \mathrm{mmol}, 1.17$ equiv.) was added under inert atmosphere. The reaction vessel was removed from the glove-box and cooled to $-40{ }^{\circ} \mathrm{C}$. Toluene (degassed by bubbling a balloon of argon for 40 min prior to use; $10 \mathrm{~mL}$ ) was then added at $-40{ }^{\circ} \mathrm{C}$, and the mixture was stirred at the same temperature for 3 hours. The mixture was then filtered on $\mathrm{SiO}_{2}$ pad and concentrated under reduced pressure. The crude was purified by column chromatography (pentane:EtOAc, 10:0 to 9:1 unless specified otherwise) affording alkynyl sulfoxides 10a-10n, 10p and 10q.

NB: Liquid substrates $(\mathbf{6 b}, \mathbf{6 i - 6 k})$ were added simultaneously with toluene and not charged into the empty flask. Degradation of the substrate could be observed due to solvation of $\mathrm{KO} t \mathrm{Bu}$.

\section{General procedure H: Synthesis of heteroaryl and alkyl alkynyl sulfoxides (10m-10o)}

TIPS-EBX (9a) (0.5 mmol, 1 equiv.) was introduced into an oven-dried two-neck $25 \mathrm{~mL}$ flask equipped with a magnetic stirrer. The reaction vessel was evacuated and backfilled with nitrogen (3 times). The reaction vessel was transferred into a glove-box where potassium tert-butoxide (0.59 mmol, 1.2 equiv.) was added under inert atmosphere. The reaction vessel was removed from the glove-box and cooled to $40{ }^{\circ} \mathrm{C}$. Toluene (degassed by bubbling a balloon of argon for $40 \mathrm{~min}$ prior to use; $10 \mathrm{~mL}$ ) was then added 
at $-40{ }^{\circ} \mathrm{C}$ simultaneously with the tert-butyl 3-(sulfinyl) propanoate (6m-6o) $(0.56 \mathrm{mmol}, 1.11$ equiv.).

The mixture was stirred at the same temperature for 2 hours, the cryostat maintaining the cooling bath was turned off and the reaction mixture was left to warm to rt over a period of 18 hours. The mixture was then filtered on $\mathrm{SiO}_{2}$ pad and concentrated under reduced pressure. The crude was purified by column chromatography (pentane:EtOAc, 10:0 to 9:1 unless specified otherwise) affording alkynyl sulfoxides

\section{0m-o.}

\section{Triisopropyl((4-methylphenylsulfinyl)ethynyl)silane (10a)}

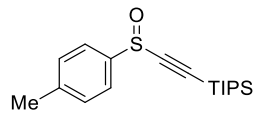

Following the general procedure $\mathbf{G}, \mathbf{1 0 a}$ was obtained as a yellow oil from $\mathbf{6 a}(0.150$ g, $0.560 \mathrm{mmol}), 9 \mathrm{a}(0.216 \mathrm{~g}, 0.504 \mathrm{mmol})$ and potassium tert-butoxide $(0.066 \mathrm{~g}, 0.59$ mmol). Yield: $87 \%(0.141 \mathrm{~g}, 0.440 \mathrm{mmol}) .{ }^{1} \mathbf{H}-\mathbf{N M R}\left(400 \mathrm{MHz}, \mathrm{CDCl}_{3}\right) \delta=7.88(\mathrm{dt}, J=8.0,1.9 \mathrm{~Hz}$, 2H), $7.35(\mathrm{~d}, J=8.0 \mathrm{~Hz}, 2 \mathrm{H}), 2.45(\mathrm{~s}, 3 \mathrm{H}), 1.13-1.03(\mathrm{~m}, 21 \mathrm{H})$ ppm. ${ }^{13} \mathbf{C}\left\{{ }^{1} \mathbf{H}\right\}-\mathbf{N M R}\left(101 \mathrm{MHz}, \mathrm{CDCl}_{3}\right)$ $\delta=142.3,141.0,130.1,125.3,108.0,102.3,21.5,18.4,10.9$ ppm. HRMS (ESI) calcd. for $\mathrm{C}_{18} \mathrm{H}_{29} \mathrm{OSSi}^{+}$ $[\mathrm{M}+\mathrm{H}]^{+}$321.1703; found 321.1707. IR (film): $\widetilde{v}=2941$ (s), 2867 (s), 1464 (s), 1384 (w), 1074 (s), 996 (m), $921(\mathrm{w}), 883(\mathrm{~s}), 810(\mathrm{w}) \mathrm{cm}^{-1}$.

\section{Triisopropyl((phenylsulfinyl)ethynyl)silane (10b)}

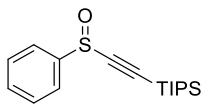

Following the general procedure $\mathbf{G}, \mathbf{1 0 b}$ was obtained as a colorless oil from $\mathbf{6 b}(0.141 \mathrm{~g}$, $0.560 \mathrm{mmol}), 9 \mathrm{a}(0.214 \mathrm{~g}, 0.500 \mathrm{mmol})$ and potassium tert-butoxide $(0.065 \mathrm{~g}, 0.59 \mathrm{mmol})$. Yield: 84\% (0.129 g, $0.420 \mathrm{mmol}) .{ }^{1} \mathbf{H}-\mathbf{N M R}\left(400 \mathrm{MHz}, \mathrm{CDCl}_{3}\right) \delta=7.93-7.77(\mathrm{~m}, 2 \mathrm{H}), 7.93-7.77(\mathrm{~m}$, 3H), $1.15-1.02(\mathrm{~m}, 21 \mathrm{H}) \mathrm{ppm} .{ }^{13} \mathbf{C}\left\{{ }^{1} \mathbf{H}\right\}-\mathbf{N M R}\left(101 \mathrm{MHz}, \mathrm{CDCl}_{3}\right) \delta=144.2,131.7,129.5,125.1,108.4$, 102.3, 18.4, 11.0 ppm. HRMS (ESI) calcd. for $\mathrm{C}_{17} \mathrm{H}_{27} \mathrm{OSSi}^{+}[\mathrm{M}+\mathrm{H}]^{+}$307.1552; found 307.1546. IR (film): $\widetilde{v}=2947$ (s), 2892 (m), 2866 (s), 1464 (m), 1445 (m), 1385 (w), 1092 (s), 1057 (s), 1021 (w), 997 (m), $884(\mathrm{~m}) \mathrm{cm}^{-1}$.

\section{Triisopropyl((4-methoxyphenylsulfinyl)ethynyl)silane (10c)}




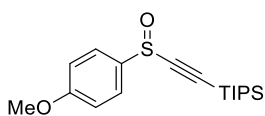

Following general procedure $\mathbf{G}, \mathbf{1 0 c}$ was obtained as a yellow oil from $\mathbf{6 c}(0.158 \mathrm{~g}$,

$0.556 \mathrm{mmol}), 9 \mathrm{a}(0.214 \mathrm{~g}, 0.500 \mathrm{mmol})$ and potassium tert-butoxide $(0.065 \mathrm{~g}, 0.59$

mmol). Yield: 90\% (0.151 g, $0.450 \mathrm{mmol}) .{ }^{1} \mathbf{H}-\mathbf{N M R}\left(400 \mathrm{MHz}, \mathrm{CDCl}_{3}\right) \delta=7.88(\mathrm{dt}, J=8,1.9 \mathrm{~Hz}, 2 \mathrm{H})$, $7.35(\mathrm{~d}, J=8.0 \mathrm{~Hz}, 2 \mathrm{H}), 2.45(\mathrm{~s}, 3 \mathrm{H}), 1.13-1.03(\mathrm{~m}, 21 \mathrm{H}) \operatorname{ppm} .{ }^{13} \mathbf{C}\left\{{ }^{1} \mathbf{H}\right\}-\mathbf{N M R}\left(101 \mathrm{MHz}, \mathrm{CDCl}_{3}\right) \delta=$ 162.6, 135.7, 127.8, 115.0, 108.0, 102.5, 55.7, 18.6, 11.1 ppm. HRMS (ESI) calcd. for $\mathrm{C}_{18} \mathrm{H}_{28} \mathrm{O}_{2} \mathrm{NaSSi}^{+}$ $[\mathrm{M}+\mathrm{Na}]^{+}$359.1477; found 359.1482. IR (film): $\widetilde{v}=3003(\mathrm{w}), 2978(\mathrm{w}), 2935(\mathrm{w}), 2837(\mathrm{w}), 1729(\mathrm{~s})$, 1594 (w), 1495 (s), 1463 (w), 1367 (w), $1286(\mathrm{~m}), 1247$ (s), $1172(\mathrm{~m}), 1150(\mathrm{~s}), 1034(\mathrm{~m}), 830(\mathrm{~m}) \mathrm{cm}^{-1}$.

\section{Triisopropyl((4-bromophenylsulfinyl)ethynyl)silane (10d)}

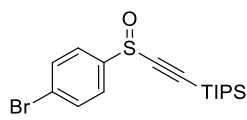

Following the general procedure $\mathbf{G}, \mathbf{1 0 d}$ was Obtained as a colorless oil from $\mathbf{6 d}(0.176$ g, $0.528 \mathrm{mmol}), 9 \mathrm{a}(0.204 \mathrm{~g}, 0.475 \mathrm{mmol})$ and potassium tert-butoxide $(0.062 \mathrm{~g}, 0.56$ mmol). Yield: 55\% (0.101 g, $0.261 \mathrm{mmol}) .{ }^{1} \mathbf{H}-\mathbf{N M R}\left(400 \mathrm{MHz}, \mathrm{CDCl}_{3}\right) \delta=7.67(\mathrm{~s}, 4 \mathrm{H}), 1.13-1.02(\mathrm{~m}$, 21H) ppm. ${ }^{13} \mathbf{C}\left\{{ }^{1} \mathbf{H}\right\}-N M R\left(101 \mathrm{MHz}, \mathrm{CDCl}_{3}\right) \delta=143.4,132.8,126.6,126.4,109.2,101.8,18.5,11.1$ ppm. HRMS (ESI) calcd. for $\mathrm{C}_{17} \mathrm{H}_{26}{ }^{79} \mathrm{BrOSSi}^{+}[\mathrm{M}+\mathrm{H}]^{+}$385.0657; found 385.0654. IR (film): $\widetilde{v}=2946$

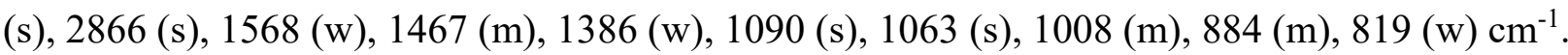

\section{Triisopropyl((4-fluorophenylsulfinyl)ethynyl)silane (10e)}

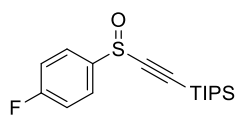

Following the general procedure $\mathbf{G}, \mathbf{1 0 e}$ was obtained as a yellow oil from $6 \mathrm{e}(0.151 \mathrm{~g}$, $0.556 \mathrm{mmol}), 9 \mathrm{a}(0.214 \mathrm{~g}, 0.500 \mathrm{mmol})$ and potassium tert-butoxide $(0.065 \mathrm{~g}, 0.59$ mmol). Yield: 75\% (0.122 g, $0.376 \mathrm{mmol}) .{ }^{1} \mathbf{H}-\mathbf{N M R}\left(400 \mathrm{MHz}, \mathrm{CDCl}_{3}\right) \delta=7.82(\mathrm{~m}, 2 \mathrm{H}), 7.23(\mathrm{~m}, 2 \mathrm{H})$, $1.29-0.75(\mathrm{~m}, 21 \mathrm{H}){ }^{13} \mathbf{C}\left\{{ }^{1} \mathbf{H}\right\}-N M R\left(101 \mathrm{MHz}, \mathrm{CDCl}_{3}\right) \delta=164.7(\mathrm{~d}, J=252.8 \mathrm{~Hz}), 139.9(\mathrm{~d}, J=3.2$ Hz), $127.6(\mathrm{~d}, J=9.1 \mathrm{~Hz}), 116.8(\mathrm{~d}, J=22.7 \mathrm{~Hz}), 108.8,102.1,18.4,11.0$ ppm. ${ }^{19} \mathbf{F}$ NMR $(400 \mathrm{MHz}$, $\left.\mathrm{CDCl}_{3}\right) \delta=-107.2(\mathrm{~s}, 1 \mathrm{~F})$ ppm. HRMS (ESI) calcd. for $\mathrm{C}_{17} \mathrm{H}_{26} \mathrm{FOSSi}^{+}[\mathrm{M}+\mathrm{H}]^{+} 325.1452$; found 325.1451. IR (film): $\widetilde{v}=2946$ (s), 2891 (m), 2866 (s), 1589 (m), 1491 (s), 1465 (m), 1233 (s), 1153 (m), 1087 (s), 1062 (s), 1014 (w), 998 (m), 882 (s), 835 (s) cm 


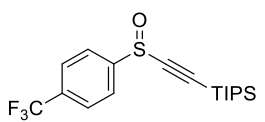

Following general procedure G, 10f was obtained as a yellow oil from $\mathbf{6 f}(0.107 \mathrm{~g}$,

$0.333 \mathrm{mmol}), 9 \mathrm{a}(0.129 \mathrm{~g}, 0.300 \mathrm{mmol})$ and potassium tert-butoxide $(0.039 \mathrm{~g}, 0.35$ mmol). Purification: full pentane. Yield: $62 \%(0.070 \mathrm{~g}, 0.19 \mathrm{mmol}) .{ }^{1} \mathbf{H}-\mathbf{N M R}\left(400 \mathrm{MHz}, \mathrm{CDCl}_{3}\right) \delta=$ $7.92(\mathrm{~d}, J=8.3 \mathrm{~Hz}, 2 \mathrm{H}), 7.81(\mathrm{~d}, J=8.3 \mathrm{~Hz}, 2 \mathrm{H}), 1.24-0.88(\mathrm{~m}, 21 \mathrm{H}) \mathrm{ppm} .{ }^{13} \mathbf{C}\left\{{ }^{1} \mathbf{H}\right\}-\mathbf{N M R}(101 \mathrm{MHz}$, $\left.\mathrm{CDCl}_{3}\right) \delta=148.3,133.5(\mathrm{q}, J=32.7 \mathrm{~Hz}), 126.5(\mathrm{q}, J=3.9 \mathrm{~Hz}), 125.2,123.4(\mathrm{q}, J=272.8 \mathrm{~Hz}), 109.8$, 101.6, 18.4, 11.0 ppm. ${ }^{19}$ F NMR $\left(376 \mathrm{MHz}_{2} \mathrm{CDCl}_{3}\right) \delta=-62.9$ ppm. HRMS (ESI) calcd. for $\mathrm{C}_{18} \mathrm{H}_{25} \mathrm{~F}_{3} \mathrm{Na}_{-}$ $\mathrm{OSSi}^{+}[\mathrm{M}+\mathrm{Na}]^{+}$397.1240; found 397.1235. IR (film): $\widetilde{v}=2936(\mathrm{w}), 2869(\mathrm{w}), 2102(\mathrm{w}), 2065$ (w), 1605 (w), $1464(\mathrm{w}), 1323(\mathrm{~s}), 1170(\mathrm{~m}), 1133(\mathrm{~m}), 1095(\mathrm{~m}), 1060(\mathrm{~s}), 1012(\mathrm{~m}), 884(\mathrm{~m}), 841(\mathrm{~m}), 765(\mathrm{~s}) \mathrm{cm}^{-}$ 1

\section{Triisopropyl((4-nitrosulfinyl)ethynyl)silane (10g)}

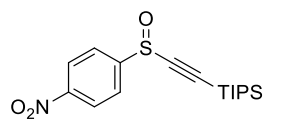

Following the general procedure $\mathbf{G}, \mathbf{1 0 g}$ was obtained as an off-white solid from $\mathbf{6 g}$

$(0.100 \mathrm{~g}, 0.333 \mathrm{mmol}), \mathbf{9 a}(0.129 \mathrm{~g}, 0.300 \mathrm{mmol})$ and potassium tert-butoxide $(0.039$ g, $0.35 \mathrm{mmol})$. Yield: 62\% (0.065 g, $0.19 \mathrm{mmol}) . \mathbf{m p}:\left({ }^{\circ} \mathrm{C}\right)$ 51.3-52.0. ${ }^{1} \mathbf{H}-\mathbf{N M R}\left(400 \mathrm{MHz}, \mathrm{CDCl}_{3}\right) \delta=$ $8.41(\mathrm{~m}, 2 \mathrm{H}), 7.97(\mathrm{~m}, 2 \mathrm{H}), 1.25-0.92(\mathrm{~m}, 21 \mathrm{H}) \mathrm{ppm} .{ }^{13} \mathbf{C}\left\{{ }^{1} \mathbf{H}\right\}-\mathbf{N M R}\left(101 \mathrm{MHz}, \mathrm{CDCl}_{3}\right) \delta=151.1$, 149.7, 125.6, 124.6, 110.7, 101.1, 18.4, 11.0 ppm. HRMS (ESI) calcd. for $\mathrm{C}_{17} \mathrm{H}_{26} \mathrm{NO}_{3} \mathrm{SSi}^{+}[\mathrm{M}+\mathrm{H}]^{+}$352.1397; found 352.1380. IR (film): $\widetilde{v}=3095$ (w), 2939 (m), 2864 (m), 1520 (s), $1460(\mathrm{~m}), 1345$ (s), 1088 (s), 1063 (s), 992 (w), 879 (m), 851 (m), 768 (s), 678 (s) cm . $^{-1}$

\section{Triisopropyl((2-bromophenylsulfinyl)ethynyl)silane (10h)}

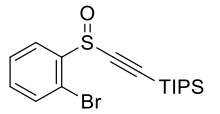

Following the general procedure $\mathbf{G}, \mathbf{1 0 h}$ was obtained as a yellow oil from $\mathbf{6 h}(0.185 \mathrm{~g}$, $0.560 \mathrm{mmol}), 9 \mathrm{a}(0.214 \mathrm{~g}, 0.500 \mathrm{mmol})$ and potassium tert-butoxide $(0.065 \mathrm{~g}, 0.58 \mathrm{mmol})$. Yield 81\% (0.156 g, $0.405 \mathrm{mmol}) .{ }^{1} \mathrm{H}-\mathrm{NMR}\left(400 \mathrm{MHz}, \mathrm{CDCl}_{3}\right) \delta=8.02(\mathrm{dd}, J=7.9,1.6 \mathrm{~Hz}, 1 \mathrm{H}), 7.69$ - $7.50(\mathrm{~m}, 2 \mathrm{H}), 7.39(\mathrm{td}, J=7.7,1.7 \mathrm{~Hz}, 1 \mathrm{H}), 1.11-0.97(\mathrm{~m}, 21 \mathrm{H}) \mathrm{ppm} .{ }^{13} \mathbf{C}\left\{{ }^{1} \mathbf{H}\right\}-\mathbf{N M R}(101 \mathrm{MHz}$, $\left.\mathrm{CDCl}_{3}\right) \delta=143.4,133.3,132.5,128.6,126.0,119.0,107.2,100.8,18.4,11.0 \mathrm{ppm}$. HRMS calcd. for 
$\mathrm{C}_{17} \mathrm{H}_{26}{ }^{79} \mathrm{BrOSSi}^{+}[\mathrm{M}+\mathrm{H}]^{+}$385.0652; found 385.0655. IR (film): $\widetilde{v}=2963$ (m), 2887 (w), 2195 (w), 2164 (w), 1594 (m), 1495 (m), 1300 (m), $1086(\mathrm{~s}), 1057$ (s), $831(\mathrm{~m}), 768(\mathrm{~m}) \mathrm{cm}^{-1}$.

\section{Triisopropyl((3-methylphenylsulfinyl)ethynyl)silane (10i)}

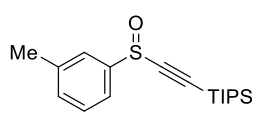

Following the general procedure G, 10i was obtained as a yellow oilfrom $\mathbf{6 i}(0.149 \mathrm{~g}$,

$0.560 \mathrm{mmol}), 9 \mathrm{a}(0.214 \mathrm{~g}, 0.500 \mathrm{mmol})$ and potassium tert-butoxide $(0.065 \mathrm{~g}, 0.58$ mmol). Yield: $75 \%(0.120 \mathrm{~g}, 0.374 \mathrm{mmol}) .{ }^{1} \mathbf{H}-\mathbf{N M R}\left(400 \mathrm{MHz}, \mathrm{CDCl}_{3}\right) \delta=7.66(\mathrm{~m}, 1 \mathrm{H}), 7.59(\mathrm{dt}, J=$ 7.7, $1.4 \mathrm{~Hz}, 1 \mathrm{H}), 7.41(\mathrm{t}, J=7.7 \mathrm{~Hz}, 1 \mathrm{H}), 7.31(\mathrm{~m}, 1 \mathrm{H}), 2.43(\mathrm{~s}, 3 \mathrm{H}), 1.20-0.97(\mathrm{~m}, 21 \mathrm{H}) \mathrm{ppm} .{ }^{13} \mathbf{C}\left\{{ }^{1} \mathbf{H}\right\}-$ NMR $\left(101 \mathrm{MHz}, \mathrm{CDCl}_{3}\right) \delta=144.0,139.7,132.4,129.2,125.5,122.3,108.1,102.4,21.4,18.4,11.0 \mathrm{ppm}$. HRMS (ESI) calcd. for $\mathrm{C}_{18} \mathrm{H}_{28} \mathrm{NaOSSi}^{+}[\mathrm{M}+\mathrm{Na}]^{+}$343.1522; found 343.1506. IR (film): $\widetilde{v}=2938$ (w), $2868(\mathrm{w}), 1466(\mathrm{w}), 1091(\mathrm{~m}), 1066(\mathrm{~m}), 882(\mathrm{~m}), 761(\mathrm{~s}), 685(\mathrm{~s}) \mathrm{cm}^{-1}$.

\section{Triisopropyl((3-bromophenylsulfinyl)ethynyl)silane (10j)}

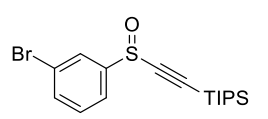

Following the general procedure $\mathbf{G}, \mathbf{1 0 j}$ was obtained as a yellow oil from $\mathbf{6 j}(0.185 \mathrm{~g}$,

$0.560 \mathrm{mmol}) \mathbf{9 b}(0.214 \mathrm{~g}, 0.500 \mathrm{mmol})$ and potassium tert-butoxide $(0.065 \mathrm{~g}, 0.59$ mmol). Yield: $37 \%(0.071 \mathrm{~g}, 0.18 \mathrm{mmol}) .{ }^{1} \mathbf{H}-\mathbf{N M R}\left(400 \mathrm{MHz}, \mathrm{CDCl}_{3}\right) \delta=7.97(\mathrm{q}, J=1.7 \mathrm{~Hz}, 1 \mathrm{H}), 7.71$ $(\mathrm{dt}, J=7.8,1.4 \mathrm{~Hz}, 1 \mathrm{H}), 7.65(\mathrm{ddd}, J=8.0,1.9,1.0 \mathrm{~Hz}, 1 \mathrm{H}), 7.41(\mathrm{t}, J=7.9 \mathrm{~Hz}, 1 \mathrm{H}), 1.17-0.98(\mathrm{~m}$, 21H) ppm. ${ }^{13} \mathbf{C}\left\{{ }^{1} \mathbf{H}\right\}-\mathbf{N M R}\left(101 \mathrm{MHz}, \mathrm{CDCl}_{3}\right) \delta=146.2,134.6,130.9,127.9,123.5,109.4,101.8,18.4$, 11.0 ppm. One carbon is not resolved. HRMS calcd. for $\mathrm{C}_{17} \mathrm{H}_{26}{ }^{79} \mathrm{BrOSSi}^{+}[\mathrm{M}+\mathrm{H}]^{+} 385.0657$; found 385.0658. IR (film): $\widetilde{v}=2893(\mathrm{~m}), 2866(\mathrm{~s}), 1569(\mathrm{~m}), 1461(\mathrm{~s}), 1407(\mathrm{~m}), 1238(\mathrm{w}), 1155(\mathrm{w}), 1091(\mathrm{~s})$, $1068(\mathrm{~s}), 1018(\mathrm{w}), 996(\mathrm{~m}), 921(\mathrm{w}), 883(\mathrm{~s}), 2944(\mathrm{~s}) \mathrm{cm}^{-1}$.

\section{Triisopropyl((pyridin-2-ylsulfinyl)ethynyl)silane (10k)}

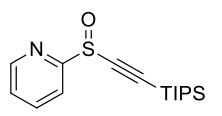

Following the general procedure $\mathbf{G}, \mathbf{1 0 k}$ was obtained as an orange oil from $6 \mathbf{k}(0.142 \mathrm{~g}$,

$0.560 \mathrm{mmol}), 9 \mathrm{a}(0.214 \mathrm{~g}, 0.500 \mathrm{mmol})$ and potassium tert-butoxide $(0.065 \mathrm{~g}, 0.58 \mathrm{mmol})$.

Yield: 85\% (0.129 g, $0.419 \mathrm{mmol}) .{ }^{1} \mathbf{H}-\mathbf{N M R}\left(400 \mathrm{MHz}, \mathrm{CDCl}_{3}\right) \delta=8.65(\mathrm{dt}, J=4.8,1.3 \mathrm{~Hz}, 1 \mathrm{H}), 8.08$ $(\mathrm{dt}, J=7.9,1.1 \mathrm{~Hz}, 1 \mathrm{H}), 7.97(\mathrm{td}, J=7.7,1.8 \mathrm{~Hz}, 1 \mathrm{H}), 7.43(\mathrm{ddd}, J=7.5,4.7,1.2 \mathrm{~Hz}, 1 \mathrm{H}), 1.15-0.95$ 
(m, 21H) ppm. ${ }^{13} \mathbf{C}\left\{{ }^{1} \mathbf{H}\right\}-N M R\left(101 \mathrm{MHz}, \mathrm{CDCl}_{3}\right) \delta=163.7,149.9,138.4,125.4,119.3,07.9,101.8,18.4$, 10.9 ppm. IR (film): $\widetilde{v}=2947$ (m), 2868 (m), 1576 (w), 1456 (m), 1422 (m), 1119 (w), 1092 (s), 1060 (s), 990 (m), 920 (w), 882 (s) cm cm $^{-1}$ HRMS (ESI) calcd. for $\mathrm{C}_{16} \mathrm{H}_{25} \mathrm{NNaOSSi}^{+}[\mathrm{M}+\mathrm{Na}]^{+}$330.1318; found 330.1324.

\section{Triisopropyl((thiophen-2-ylsulfinyl)ethynyl)silane (101)}

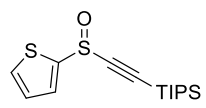

Following the general procedure G, 101 was obtained as a yellow oil from $\mathbf{6 1}(0.087 \mathrm{~g}$, $0.33 \mathrm{mmol}), 9 \mathrm{a}(0.129 \mathrm{~g}, 0.300 \mathrm{mmol})$ and potassium tert-butoxide $(0.039 \mathrm{~g}, 0.35 \mathrm{mmol})$.

Yield: 83\% (0.078 g, $0.25 \mathrm{mmol}) .{ }^{1} \mathbf{H}-\mathbf{N M R}\left(400 \mathrm{MHz}, \mathrm{CDCl}_{3}\right) \delta=7.69(\mathrm{dd}, J=5.0,1.3 \mathrm{~Hz}, 1 \mathrm{H}), 7.63$ $(\mathrm{dd}, J=3.7,1.3 \mathrm{~Hz}, 1 \mathrm{H}), 7.11(\mathrm{dd}, J=5.0,3.7 \mathrm{~Hz}, 1 \mathrm{H}), 1.32-0.98(\mathrm{~m}, 21 \mathrm{H}) \mathrm{ppm} .{ }^{13} \mathbf{C}\left\{{ }^{1} \mathbf{H}\right\}-\mathbf{N M R}(101$ $\left.\mathrm{MHz}_{2} \mathrm{CDCl}_{3}\right) \delta=145.8,132.8,132.2,127.6,108.6,101.6,18.5,11.1 \mathrm{ppm}$. HRMS (ESI) calcd. for $\mathrm{C}_{15} \mathrm{H}_{25} \mathrm{OSS}_{2} \mathrm{Si}^{+}[\mathrm{M}+\mathrm{H}]^{+}$313.1111; found 313.1096. IR (film): $\widetilde{v}=3070(\mathrm{w}), 3039(\mathrm{w}), 2951(\mathrm{w}), 2857(\mathrm{w})$, $1466(\mathrm{w}), 1322(\mathrm{w}), 1222(\mathrm{w}), 1091(\mathrm{w}), 1072(\mathrm{~m}), 997(\mathrm{~m}), 885(\mathrm{w}), 850(\mathrm{w}), 762(\mathrm{~s}) \mathrm{cm}^{-1}$.

\section{Triisopropyl((2-methylfuran-3-ylsulfinyl)ethynyl)silane (10m)}

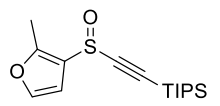

Following the general procedure $\mathbf{H}, \mathbf{1 0 m}$ was obtained as a yellow oil from $\mathbf{6 m}(0.086 \mathrm{~g}$, $0.33 \mathrm{mmol}), 9 \mathbf{a}(0.129 \mathrm{~g}, 0.300 \mathrm{mmol})$ and potassium tert-butoxide $(0.039 \mathrm{~g}, 0.35 \mathrm{mmol})$.

Yield: 91\% (0.085 g, $0.27 \mathrm{mmol}) .{ }^{1} \mathbf{H}-\mathbf{N M R}\left(400 \mathrm{MHz}, \mathrm{CDCl}_{3}\right) \delta=7.36(\mathrm{~d}, J=2.1 \mathrm{~Hz}, 1 \mathrm{H}), 6.81(\mathrm{~d}, J=$ $2.1 \mathrm{~Hz}, 1 \mathrm{H}), 2.51(\mathrm{~s}, 3 \mathrm{H}), 1.23-1.04(\mathrm{~m}, 21 \mathrm{H}) \mathrm{ppm} .{ }^{13} \mathbf{C}\left\{{ }^{1} \mathbf{H}\right\}-\mathbf{N M R}\left(101 \mathrm{MHz}, \mathrm{CDCl}_{3}\right) \delta=155.7,142.2$ 124.4, 108.6, 107.0, 101.1, 18.5, 12.3, $11.0 \quad$ ppm. HRMS (APPI) calcd. for $\mathrm{C}_{16} \mathrm{H}_{27} \mathrm{O}_{2} \mathrm{SSi}^{+o}[\mathrm{M}]^{+\circ}$ 311.1496; found 311.1490. IR (film): $\widetilde{v}=2942(\mathrm{w}), 2861(\mathrm{w}), 1592(\mathrm{w}), 1523(\mathrm{w})$, $1466(\mathrm{w}), 1385(\mathrm{w}), 1227(\mathrm{w}), 1070(\mathrm{~s}), 882(\mathrm{~m}), 765(\mathrm{~s}), 680(\mathrm{~m}), 655(\mathrm{~m}) \mathrm{cm}^{-1}$.

\section{Triisopropyl((pentylsulfinyl)ethynyl)silane (10n)}

Following the general procedure $\mathbf{H}, \mathbf{1 0}$ was obtained as a pale yellow oil from $6 \mathbf{n}$ $(0.138 \mathrm{~g}, 0.556 \mathrm{mmol}) 9 \mathrm{a}(0.214 \mathrm{~g}, 0.500 \mathrm{mmol})$ and potassium tert-butoxide $(0.065 \mathrm{~g}, 0.59 \mathrm{mmol}, 1.1$ equiv.). Yield: 77\% (0.115 g, $0.383 \mathrm{mmol}) .{ }^{1} \mathbf{H}-\mathbf{N M R}\left(400 \mathrm{MHz}, \mathrm{CDCl}_{3}\right) \delta=3.07(\mathrm{dt}, J=8.2,6.8 \mathrm{~Hz}$, 
2H), 1.98-1.81 (m, 2H), 1.53-1.30 (m, 4H), 1.20-1.02 (m, 21H), $0.92(\mathrm{t}, J=7.2 \mathrm{~Hz}, 3 \mathrm{H})$ ppm. ${ }^{13} \mathbf{C}\left\{{ }^{1} \mathbf{H}\right\}-$ NMR $\left(101 \mathrm{MHz} \mathrm{CDCl}_{3}\right) \delta=107.3,101.6,56.5,30.6,22.3,22.1,18.5,13.8,11.0$ ppm. HRMS (ESI) calcd. for $\mathrm{C}_{16} \mathrm{H}_{32} \mathrm{OSSi}^{+}[\mathrm{M}+\mathrm{H}]^{+}$301.2021; found 301.2021. IR (film): $\widetilde{v}=2941$ (s), 2866 (s), 1464 (m), $1384(\mathrm{w}), 1075(\mathrm{~s}), 997(\mathrm{w}), 922(\mathrm{w}), 883(\mathrm{~s}) \mathrm{cm}^{-1}$.

\section{Triisopropyl((cyclohexylsulfinyl)ethynyl)silane (100)}

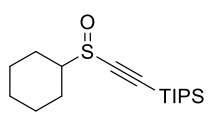

Following the general procedure $\mathbf{H}, \mathbf{1 0 0}$ was obtained as a pale yellow oil from $\mathbf{6 0}(0.145$

g, $0.556 \mathrm{mmol}) 9 \mathrm{a}(0.214 \mathrm{~g}, 0.500 \mathrm{mmol})$ and potassium tert-butoxide $(0.065 \mathrm{~g}, 0.59 \mathrm{mmol}$, 1.1 equiv.). Yield: $79 \%$ (0.123 g, $0.393 \mathrm{mmol}) .{ }^{1} \mathbf{H}-\mathbf{N M R}\left(400 \mathrm{MHz}, \mathrm{CDCl}_{3}\right) \delta=2.95$ (tt, $J=11.7,3.6$ $\mathrm{Hz}, 1 \mathrm{H}), 2.22(\mathrm{dqd}, J=12.8,3.6,1.8 \mathrm{~Hz}, 1 \mathrm{H}), 2.13(\mathrm{dtq}, J=10.4,3.4,1.9 \mathrm{~Hz}, 1 \mathrm{H}), 1.93$ (dddd, $J=14.6$, 7.8, 4.1, 1.4 Hz, 2H), $1.84-1.69(\mathrm{~m}, 1 \mathrm{H}), 1.64-1.01(\mathrm{~m}, 26 \mathrm{H})$ ppm. ${ }^{13} \mathbf{C}\left\{{ }^{1} \mathbf{H}\right\}-\mathbf{N M R}\left(101 \mathrm{MHz}, \mathrm{CDCl}_{3}\right)$ $\delta=107.8,100.4,63.6,25.7,25.5,25.5,25.5,25.4,18.5,11.0$ ppm. HRMS (ESI) $[\mathrm{M}+\mathrm{Na}]^{+}$calcd. for $\mathrm{C}_{17} \mathrm{H}_{32} \mathrm{NaOSSi}^{+}[\mathrm{M}+\mathrm{Na}]^{+}$335.1835; found 335.1820. IR (film): $\widetilde{v}=2945$ (m), 2863 (m), 1456 (w), 1065 (m), $996(\mathrm{w}), 883(\mathrm{~m}), 763(\mathrm{~s}), 674(\mathrm{~m}) \mathrm{cm}^{-1}$.

\section{Tertbutyldiphenyl((4-methylphenylsulfinyl)ethynyl)silane (10p)}

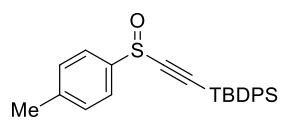

Following the general procedure $\mathbf{G}, \mathbf{1 0 p}$ was obtained as a yellow oil from $\mathbf{6 p}(0.087$ $\mathrm{g}, 0.32 \mathrm{mmol}), 9 \mathrm{~b}(0.149 \mathrm{~g}, 0.292 \mathrm{mmol})$ and potassium tert-butoxide $(0.038 \mathrm{~g}, 0.34$ mmol). Yield: 81\% (0.095 g, $0.24 \mathrm{mmol}) .{ }^{1} \mathbf{H}-\mathbf{N M R}\left(400 \mathrm{MHz}, \mathrm{CDCl}_{3}\right) \delta=7.81-7.74(\mathrm{~m}, 2 \mathrm{H}), 7.68$ (ddd, $J=6.8,3.8,1.9 \mathrm{~Hz}, 4 \mathrm{H}), 7.46-7.38(\mathrm{~m}, 2 \mathrm{H}), 7.40-7.31(\mathrm{~m}, 6 \mathrm{H}), 2.45(\mathrm{~s}, 3 \mathrm{H}), 1.07(\mathrm{~s}, 9 \mathrm{H}) \mathrm{ppm}$. ${ }^{13} \mathbf{C}\left\{{ }^{1} \mathbf{H}\right\}$-NMR $\left(101 \mathrm{MHz}, \mathrm{CDCl}_{3}\right) \delta=142.7,140.8,135.5,131.3,130.3,130.1,128.0,125.4,106.1$, 104.5, 26.9, 21.6, 18.8 ppm. HRMS (APPI) calcd. for $\mathrm{C}_{25} \mathrm{H}_{27} \mathrm{OSSi}^{+}[\mathrm{M}+\mathrm{H}]^{+}$403.1546; found 403.1541. IR (film): $\widetilde{v}=3076$ (w), 2939 (w), 2927 (w), 2858 (w), 1589 (w), 1464 (w), 1427 (w), 1389 (w), 1196 (w), 1109 (m), $1091(\mathrm{~m}), 1060(\mathrm{~m}), 1010(\mathrm{w}), 811(\mathrm{~m}), 761$ (s), 699 (s) cm².

\section{Tertbutyl((4-methylphenylsulfinyl)ethynyl)silane (10q)}




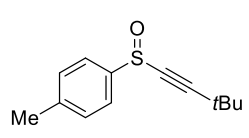

Following the general procedure $\mathbf{G}, \mathbf{1 0 q}$ was obtained as a yellow oil from $\mathbf{6 q}(0.070$

g, $0.26 \mathrm{mmol}), 9 \mathrm{c}(0.077 \mathrm{~g}, 0.24 \mathrm{mmol})$ and potassium tert-butoxide $(0.031 \mathrm{~g}, 0.27$

mmol). Yield 58\% (0.030 g, $0.14 \mathrm{mmol}) .{ }^{1} \mathbf{H}-\mathbf{N M R}\left(400 \mathrm{MHz}, \mathrm{CD}_{3} \mathrm{OD}\right) \delta=7.71(\mathrm{~d}, J=8.4 \mathrm{~Hz}, 2 \mathrm{H}), 7.46$

- $7.41(\mathrm{~m}, 2 \mathrm{H}), 2.44(\mathrm{~s}, 3 \mathrm{H}), 1.29(\mathrm{~s}, 9 \mathrm{H}) \mathrm{ppm} .{ }^{13} \mathbf{C}\left\{{ }^{1} \mathbf{H}\right\}-\mathbf{N M R}\left(101 \mathrm{MHz}, \mathrm{CD}_{3} \mathrm{OD}\right) \delta=143.1,140.6$ 130.1, 125.1, 113.4, 75.9, 28.7, 28.3, 20.1 ppm. HRMS (APPI) calcd. for $\mathrm{C}_{13} \mathrm{H}_{17} \mathrm{OS}^{+}[\mathrm{M}+\mathrm{H}]^{+}$221.0995; found 221.0993. IR (film): $\widetilde{v}=2971$ (m), 2929 (w), 2863 (w), 1597 (w), 1489 (w), 1453 (w), 1364 (w), 1252 (m), 1202 (w), 1087 (s), 1059 (s), 1017 (w), $934(\mathrm{w}), 809$ (s) $\mathrm{cm}^{-1}$.

\section{General procedure I: Synthesis of aryl alkenyl sulfoxides (12a-b)}

The tert-butyl 3-((4-methyl)sulfinyl)propanoate (6a) (0.33 mmol, 1.1 equiv.) and Ar-VBX (11a or 11b) (0.30 mmol, 1.0 equiv.) were introduced into an oven-dried two-neck $25 \mathrm{~mL}$ flask equipped with a magnetic stirrer. The reaction vessel was evacuated and backfilled with nitrogen ( 3 times). The reaction vessel was transferred into a glove-box where potassium tert-butoxide $(0.35 \mathrm{mmol}, 1.2$ equiv. $)$ was added under inert atmosphere. The reaction vessel was removed from the glove-box and cooled to $-40{ }^{\circ} \mathrm{C}$. Toluene (degassed by bubbling a balloon of argon for $40 \mathrm{~min}$ prior to use; $10 \mathrm{~mL}$ ) was then added at $-40{ }^{\circ} \mathrm{C}$, and the mixture was stirred at the same temperature for 5 hours. The mixture was then filtered on a $\mathrm{SiO}_{2} \mathrm{pad}$ and concentrated under reduced pressure. The crude was purified by column chromatography (pentane:EtOAc, 10:0 to 9:1 unless specified otherwise) affording alkynyl sulfoxides $\mathbf{1 2 a}$ and $\mathbf{1 2 b}$.

\section{Phenyl((4-methylphenylsulfinyl)ethenyl)silane (12a)}

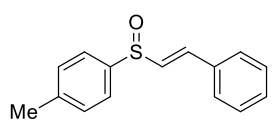

Following general procedure I, 12a was obtained as a colorless solid from 6a $(0.089$

$\mathrm{g}, 0.33 \mathrm{mmol}), 11 \mathrm{a}(0.105 \mathrm{~g}, 0.300 \mathrm{mmol})$ and potassium tert-butoxide $(0.039 \mathrm{~g}, 0.35$ mmol). Yield: $52 \%$ (0.038 g, $0.16 \mathrm{mmol}) . \mathbf{m p :} 62.1-62.4{ }^{\circ} \mathrm{C} .{ }^{1} \mathbf{H}-\mathbf{N M R}\left(400 \mathrm{MHz}, \mathrm{CDCl}_{3}\right) \delta=7.57(\mathrm{~d}$, $J=8.2 \mathrm{~Hz}, 2 \mathrm{H}), 7.47-7.42(\mathrm{~m}, 2 \mathrm{H}), 7.40-7.29(\mathrm{~m}, 6 \mathrm{H}), 6.81(\mathrm{~d}, J=15.5 \mathrm{~Hz}, 1 \mathrm{H}), 2.41(\mathrm{~s}, 3 \mathrm{H}) .{ }^{13} \mathbf{C}\left\{{ }^{1} \mathbf{H}\right\}-$ NMR $\left(101 \mathrm{MHz} \mathrm{CDCl}_{3}\right) \delta=141.9,140.8,136.2,134.0,133.2,130.3,129.9,129.0,127.9,125.1,21.6$ ppm. HRMS (APCI) calcd. for $\mathrm{C}_{15} \mathrm{H}_{15} \mathrm{OS}^{+}[\mathrm{M}+\mathrm{H}]^{+}$243.0838; found 243.0841. IR (film): $\widetilde{v}=3040$ (w), 
$2959(w), 2928$ (w), $2854(w), 1492$ (w), 1443 (w), $1080(\mathrm{~m}), 1043$ (s), 1015 (m), 952 (m), 814 (s), 734

(s) $\mathrm{cm}^{-1}$.

\section{4-Methylphenyl((4-methylphenylsulfinyl)ethenyl)silane (12b)}

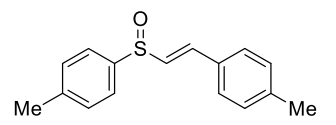

Following general procedure I, 12b was obtained as a colorless solid from $\mathbf{6 a}$

$(0.089 \mathrm{~g}, 0.33 \mathrm{mmol}), \mathbf{1 1 b}(0.109 \mathrm{~g}, 0.300 \mathrm{mmol})$ and potassium tert-butoxide $(0.039$ g, $0.35 \mathrm{mmol})$. Yield: $25 \%$ (0.020 g, $0.080 \mathrm{mmol})$. mp: 95.0 - $96.4{ }^{\circ} \mathrm{C} .{ }^{1} \mathbf{H}-\mathbf{N M R}\left(400 \mathrm{MHz}, \mathrm{CDCl}_{3}\right) \delta=$ $7.60-7.53(\mathrm{~m}, 2 \mathrm{H}), 7.37-7.28(\mathrm{~m}, 5 \mathrm{H}), 7.16(\mathrm{~d}, J=7.9 \mathrm{~Hz}, 2 \mathrm{H}), 6.76(\mathrm{~d}, J=15.5 \mathrm{~Hz}, 1 \mathrm{H}), 2.41(\mathrm{~s}, 3 \mathrm{H})$, $2.35(\mathrm{~s}, 3 \mathrm{H}) .{ }^{13} \mathbf{C}\left\{{ }^{1} \mathbf{H}\right\}$-NMR $\left(101 \mathrm{MHz}, \mathrm{CDCl}_{3}\right) \delta=141.7,140.9,140.2,136.5,132.0,131.1,130.1,129.6$, 127.8, 124.9, 21.5, 21.4 ppm. HRMS (ESI) calcd. for $\mathrm{C}_{16} \mathrm{H}_{17} \mathrm{OS}^{+}[\mathrm{M}+\mathrm{H}]^{+}$257.0995; found 257.0999. IR (film): $\widetilde{v}=3013(\mathrm{w}), 2920(\mathrm{w}), 2846(\mathrm{w}), 1513(\mathrm{w}), 1486(\mathrm{w}), 1440(\mathrm{w}), 1173(\mathrm{w}), 1082(\mathrm{~m}), 1047$ (s), $980(\mathrm{~m}), 791(\mathrm{~s}) \mathrm{cm}^{-1}$.

\section{Gram-scale synthesis of 10a}

The tert-butyl 3-((4-methylphenyl)sulfinyl) propanoate (6a) (1.22 g, $4.54 \mathrm{mmol}, 1.11 \mathrm{equiv}$.$) and$ TIPS-EBX (9a) (1.74 g, 4.09 mmol, 1.0 equiv.) were introduced into an oven-dried two-neck $250 \mathrm{~mL}$ flask equipped with a magnetic stirrer. The reaction vessel was evacuated and backfilled with nitrogen (3 times). The reaction vessel was transferred into a glove-box where potassium tert-butoxide (0.535 g, 4.77 mmol, 1.17 equiv.) was added under inert atmosphere. The reaction vessel was removed from the glovebox and cooled to $-40{ }^{\circ} \mathrm{C}$. Toluene (degassed by bubbling a balloon of argon for 40 min prior to use; 80 $\mathrm{mL}$ ) was then added at $-40{ }^{\circ} \mathrm{C}$, and the mixture was stirred at the same temperature for 4 hours. The mixture was then filtered on $\mathrm{SiO}_{2}$ pad and concentrated under reduced pressure. The crude was purified by column chromatography (pentane:EtOAc, 10:0 to 9:1) affording pure 10a in 86\% yield (1.13 g, 3.52 mmol). ${ }^{1} \mathbf{H}-\mathbf{N M R}\left(400 \mathrm{MHz}, \mathrm{CDCl}_{3}\right) \delta=7.88(\mathrm{dt}, J=8.0,1.9 \mathrm{~Hz}, 2 \mathrm{H}), 7.35(\mathrm{~d}, J=8.0 \mathrm{~Hz}, 2 \mathrm{H}), 2.45(\mathrm{~s}$, 3H), $1.13-1.03(\mathrm{~m}, 21 \mathrm{H}) \mathrm{ppm} .{ }^{13} \mathbf{C}\left\{{ }^{1} \mathbf{H}\right\}-N M R\left(101 \mathrm{MHz}, \mathrm{CDCl}_{3}\right) \delta=142.3,141.0,130.1,125.3,108.0$, $102.3,21.5,18.4,10.9$. 


\section{Desilylation of 10a}

Following a modified reported procedure, ${ }^{43}$ in a $10 \mathrm{~mL}$ round-bottomed, single-necked

flask, triisopropyl((4-methylphenylsulfinyl)ethynyl)silane (10a) (0.127 g, 0.396 mmol, 1.0 equiv.) was dissolved in ethanol $(2.7 \mathrm{~mL})$. The resulting solution was cooled to $0{ }^{\circ} \mathrm{C}$ (ice-water bath) under stirring. An ice-cold solution of KF (0.092 g, $1.6 \mathrm{mmol}, 4.0$ equiv.) in water (1.3 $\mathrm{mL})$ was then added drop-wise. The initially pale yellow solution slowly darkened to orange. The mixture was stirred at $0{ }^{\circ} \mathrm{C}$ for 2 hours. The reaction was quenched by addition of water $(10 \mathrm{~mL})$ and the aqueous layer was then extracted with ethyl acetate $(3 \times 15 \mathrm{~mL})$. The combined organic extracts were washed with brine, dried over $\mathrm{MgSO}_{4}$, filtered and concentrated under vacuum. The resulting yellow crude oil was submitted to column chromatography ( $\mathrm{SiO}_{2} ; \mathrm{DCM}: \mathrm{MeOH}$ 1:0 to 9:1) to afford pure 1-(ethynylsulfinyl)-4methylbenzene $\mathbf{1 3}$ in $58 \%$ yield $(0.037 \mathrm{~g}, 0.23 \mathrm{mmol})$ as a colorless oil. ${ }^{1} \mathbf{H}-\mathbf{N M R}\left(400 \mathrm{MHz}, \mathrm{CDCl}_{3}\right) \delta=$ $7.71(\mathrm{~d}, J=8.3 \mathrm{~Hz}, 2 \mathrm{H}), 7.36(\mathrm{~m}, 2 \mathrm{H}), 3.71(\mathrm{~s}, 1 \mathrm{H}), 2.43(\mathrm{~s}, 3 \mathrm{H}) \mathrm{ppm} .{ }^{13} \mathbf{C}\left\{{ }^{1} \mathbf{H}\right\}-\mathbf{N M R}\left(101 \mathrm{MHz}, \mathrm{CDCl}_{3}\right)$ $\delta=142.9,140.0,130.3,125.2,90.1,81.8,21.5$ ppm. HRMS (APCI) calcd. for $\mathrm{C}_{9} \mathrm{H}_{9} \mathrm{OS}^{+}[\mathrm{M}+\mathrm{H}]^{+}$ 165.0369; found 165.0371. IR (film): $\widetilde{v}=3275$ (w), 3186 (m), 3054 (w), 2039 (m), 1089 (s), 1053 (s), $808(\mathrm{~s}), 910(\mathrm{~m}), 1017(\mathrm{~m}), 1406(\mathrm{w}), 1453(\mathrm{w}), 1489(\mathrm{w}), 1597(\mathrm{w}) \mathrm{cm}^{-1}$.

\section{Acknowledgment}

We thank ERC (European Research Council, Starting Grant iTools4MC, number 334840) and EPFL for financial support. S. G. E. A. thanks the Institute of Chemical Sciences and Engineering (ISIC) at EPFL for an ISIC master grant.

\section{Supporting Information.}

Reactions schemes for the synthesis of starting materials and reagents, reaction optimization details, accident report, and chromatogram and NMR spectra of new compounds or reported compounds synthesized using modified methods. This material is available free of charge via the Internet at http://pubs.acs.org.

\section{References}


(1) St-Gelais, A.; Legault, J.; Mshvildadze, V.; Pichette, A. Dirchromones: Cytotoxic Organic Sulfur Compounds Isolated from Dirca Palustris. J. Nat. Prod. 2015, 78, 1904-1909.

(2) a) Jacob, C.; Giles, G. I.; Giles, N. M.; Sies, H. Sulfur and Selenium: The Role of Oxidation State in Protein Structure and Function. Angew. Chem. Int. Ed. 2003, 42, 4742-4758. b) Kubota, K.; Hirayama, H.; Sato, Y.; Kobayashi, A.; Sugawara, F. Amino Acid Precursors of the Garlic-Like Odour in Scorodocarpus Borneensis. Phytochemistry 1998, 49, 99-102.

(3) Numata, M.; Aoyagi, Y.; Tsuda, Y.; Yarita, T.; Takatsu, A. Preparation of Sulfoxide Residue Bonded Silica Stationary Phase for Separation of Polychlorinated Biphenyls from Mineral Oils. Anal. Chem. 2008, 80, 509-509.

(4) McTavish, D.; Buckley, M. M.-T.; Heel, R. C. Omeprazole. Drugs 1991, 42, 138-170.

(5) a) Ramos, E. T.; Hungenberg, H.; Balabhadrapathruni, S. Patent EP 2422620 A1, 2012. b) Adler, M.; Braude, V.; Chen, K.; Moshkovits-Kaptsan, R. Patent EP 2159219 A2, 2010.

(6) a) Carreno, M. C., Applications of Sulfoxides to Asymmetric-Synthesis of Biologically-Active Compounds. Chem. Rev. 1995, 95 , 1717-1760. b) Fernandez, I.; Khiar, N., Recent developments in the synthesis and utilization of chiral sulfoxides. Chem. Rev. 2003, 103, 3651-3705. c) Otocka, S.; Kwiatkowska, M.; Madalińska, L.; Kiełbasiński, P. Chiral Organosulfur Ligands/Catalysts with a Stereogenic Sulfur Atom: Applications in Asymmetric Synthesis. Chem. Rev. 2017, 117, 4147-4181.

(7) Modern Alkyne Chemistry: Catalytic and Atom-Economic Transformations; Trost, B. M., Li, C.J., Eds.; Wiley-VCH: Weinheim, 2015.

(8) Barrett, M. J.; Khan, G. F.; Davies, P. W.; Grainger, R. S. Alkynyl Sulfoxides as $\alpha$-Sulfinyl Carbene Equivalents: Gold-Catalysed Oxidative Cyclopropanation. Chem. Commun. 2017, 53, 5733-5736.

(9) a) Varghese, J. P.; Knochel, P.; Marek, I. New Allene Synthesis via Carbocupration-Zinc Carbenoid Homologation and $\beta$-Elimination Sequence. Org. Lett. 2000, 2, 2849-2852. b) Marek, I. A Shift in Retrosynthetic Paradigm. Chem. Eur. J. 2008, 14, 7460-7468. 
(10) Bouillon, J.-P.; Musyanovich, R.; Portella, C.; Shermolovich, Y. 1-(Alkylsulfinyl- and -Alkylsulfonyl)-2-(F-Alkyl)Ethynes - Applications in Cycloaddition Reactions and Synthesis of Aromatic Derivatives. Eur. J. Org. Chem. 2001, 2001 , 3625-3629.

(11) Souza, F. B.; Shamim, A.; Argomedo, L. M. Z.; Pimenta, D. C.; Stefani, H. A. Synthesis of Enyne and Aryl Vinyl Sulfoxides: Functionalization via Pummerer Rearrangement. Mol. Divers. 2015, 19, 773785.

(12) a) Zhong, P.; Huang, X.; Ping-Guo, M. Hydrozirconation of Alkynyl Sulfoxides: The Reactions of Zirconated Vinyl Sulfoxide Intermediates. Tetrahedron 2000, 56, 8921-8925. b) Huang, X.; Duan, D.; Zheng, W. Studies on Hydrozirconation of 1-Alkynyl Sulfoxides or Sulfones and the Application for the Synthesis of Stereodefined Vinyl Sulfoxides or Sulfones. J. Org. Chem. 2003, 68, 1958-1963.

(13) a) Pfizer, Inc., Patent US5716965 A1, 1998. b) P. E. Reddy, R. M. V. Reddy, S. C. Bell, Patent WO 2005046599 A2, 2005. c) Millennium Pharmaceuticals, Inc., M. O. Duffey, D. B. England, Z. Hu, M. Ito, S. P. Langston, C. Mcintyre, H. Mizutani, H. Xu, Patent WO2015/2994 A2, 2015.

(14) a) Marino, J. P.; Neisser, M. Stereospecific Reactions of Dichloroketene with Vinyl Sulfoxides: A New Type of Polar Cycloaddition. J. Am. Chem. Soc. 1981, 103, 7687-7689. b) Westwell, A. D.; Rayner, C. M. Chapter 5: The Chemistry of $\alpha, \beta$-Unsaturated Sulfoxides. In Organosulfur Chemistry; Page, P., Ed.; Academic Press, 1998; Vol. 2, pp 157-228. c) Bur, S. K.; Padwa, A. The Pummerer Reaction: Methodology and Strategy for the Synthesis of Heterocyclic Compounds. Chem. Rev. 2004, 104, 2401-2432. d) Nishimura, T.; Unni, A. K.; Yokoshima, S.; Fukuyama, T. Concise Total Synthesis of (+)-Lyconadin A. J. Am. Chem. Soc. 2011, 133, 418-419.

(15) a) Kabanyane, S. T.; MaGee, D. I. An Efficient and General Synthesis of Alkyl Alkynyl Sulfoxides. Can. J. Chem. 1992, 70, 2758-2763. b) Davis, F. A.; Reddy, R. T.; Han, W.; Carroll, P. J. Chemistry of Oxaziridines. 17. N-(Phenylsulfonyl)(3,3-Dichlorocamphoryl)Oxaziridine: A Highly Efficient Reagent for the Asymmetric Oxidation of Sulfides to Sulfoxides. J. Am. Chem. Soc. 1992, 114, 1428-1437. c) Zhong, P.; Guo, M.-P.; Huang, N.-P., A Selective and Convenient Oxidation of Acetylenic Sulfides to Acetylenic Sulfoxides Using Trichloroisocyanuric Acid. Synth. Commun. 2002, 32, 175-179. d) Zhang, 
G.; Zhang, L. Au-Containing All-Carbon 1,3-Dipoles: Generation and [3+2] Cycloaddition Reactions. J. Am. Chem. Soc. 2008, 130, 12598-12599.

(16) Caupène, C.; Boudou, C.; Perrio, S.; Metzner, P. Remarkably Mild and Simple Preparation of Sulfenate Anions from $\beta$-Sulfinylesters: A New Route to Enantioenriched Sulfoxides. J. Org. Chem. 2005, 70, 2812-2815.

(17) O’donnell, J. S.; Schwan, A. L. Generation, Structure and Reactions of Sulfenic Acid Anions. J. Sulfur Chem. 2004, 25, 183-211.

(18) Selected examples: a) Maitro, G.; Vogel, S.; Prestat, G.; Madec, D.; Poli, G. Aryl Sulfoxides via Palladium-Catalyzed Arylation of Sulfenate Anions. Org. Lett. 2006, 8, 5951-5954. b) Maitro, G.; Vogel, S.; Sadaoui, M.; Prestat, G.; Madec, D.; Poli, G. Enantioselective Synthesis of Aryl Sulfoxides via Palladium-Catalyzed Arylation of Sulfenate Anions. Org. Lett. 2007, 9, 5493-5496. c) Gelat, F.; Jayashankaran, J.; Lohier, J.-F.; Gaumont, A.-C.; Perrio, S. Organocatalytic Asymmetric Synthesis of Sulfoxides from Sulfenic Acid Anions Mediated by a Cinchona-Derived Phase-Transfer Reagent. Org. Lett. 2011, 13, 3170-3173. d) Söderman, S. C.; Schwan, A. L., The Diastereoselective Alkylation of Arenesulfenate Anions Using Homochiral Electrophiles. Org. Lett. 2011, 13, 4192-4195. e) Zong, L.; Ban, X.; Kee, C. W.; Tan, C.-H. Catalytic Enantioselective Alkylation of Sulfenate Anions to Chiral Heterocyclic Sulfoxides Using Halogenated Pentanidium Salts. Angew. Chem. Int. Ed. 2014, 53, 11849-11853. f) Jia, T.; Zhang, M.; Jiang, H.; Wang, C. Y.; Walsh, P. J. Palladium-Catalyzed Arylation of Alkyl Sulfenate Anions. J. Am. Chem. Soc. 2015, 137, 13887-13893. g) Jia, T. Z.; Zhang, M. N.; McCollom, S. P.; Bellomo, A.; Montel, S.; Mao, J. Y.; Dreher, S. D.; Welch, C. J.; Regalado, E. L.; Williamson, R. T.; Manor, B. C.; Tomson, N. C.; Walsh, P. J., Palladium-Catalyzed Enantioselective Arylation of Aryl Sulfenate Anions: A Combined Experimental and Computational Study. J. Am. Chem. Soc. 2017, 139, 8337-8345. h) Wang, L.; Chen, M.; Zhang, P.; Li, W.; Zhang, J. Palladium/PC-Phos-Catalyzed Enantioselective Arylation of General Sulfenate Anions: Scope and Synthetic Applications. J. Am. Chem. Soc. 2018, 140, 3467-3473.

(19) a) Yu, H.; Li, Z.; Bolm, C. Transition-Metal-Free Arylations of In-Situ Generated Sulfenates with Diaryliodonium Salts. Org. Lett. 2018, 20, 7104-7106. b) Zhang, J.; Wang, L.; Chen, M. Transition Metal- 
Free Base-Promoted Arylation of General Sulfenate Anions with Diaryliodonium Salts. Org. Chem. Front. 2018, 6, 32-35.

(20) Yoshimura, A.; Zhdankin, V. V., Advances in Synthetic Applications of Hypervalent Iodine Compounds. Chem. Rev. 2016, 116, 3328-3435.

(21) Hari, D. P.; Nicolai, S.; Waser, J. Alkynylations and Vinylations. In PATAI'S Chemistry of Functional Groups; Z. Rappoport (Ed.), 2018; pp 1-58.

(22) Li, Y.; Hari, D. P.; Vita, M. V.; Waser, J., Cyclic Hypervalent Iodine Reagents for Atom-Transfer Reactions: Beyond Trifluoromethylation. Angew. Chem., Int. Ed. 2016, 55, 4436-4454.

(23) a) Frei, R.; Waser, J. A Highly Chemoselective and Practical Alkynylation of Thiols. J. Am. Chem. Soc. 2013, 135, 9620-9623. b) Frei, R.; Wodrich, M. D.; Hari, D. P.; Borin, P.-A.; Chauvier, C.; Waser, J. Fast and Highly Chemoselective Alkynylation of Thiols with Hypervalent Iodine Reagents Enabled through a Low Energy Barrier Concerted Mechanism. J. Am. Chem. Soc. 2014, 136, 16563-16573. c) Chen, C. C.; Waser, J. One-Pot, Three-Component Arylalkynyl Sulfone Synthesis. Org. Lett. 2015, 17, 736-739. d) Wodrich, M. D.; Caramenti, P.; Waser, J., Alkynylation of Thiols with Ethynylbenziodoxolone (EBX) Reagents: $\alpha$ - or $\beta$ - $\pi$-Addition? Org. Lett. 2016, 18, 60-63.

(24) Similar results were obtained with other ethynylbenziodoxole reagents and alkynyliodonium salts gave much lower yields. See Supporting Information.

(25) See experimental part for details. Precursors 5-7 were synthesized using reported procedures (ref. $18 \mathrm{~b}-\mathrm{c}$ ) by sequential Michael-addition then oxidation in respectively, $66 \%, 79 \%$ and $26 \%$ yield over two steps. 8 was synthesised via a three-step procedure: a) acetylation of nitroethanol, b) substitution of the acetylated position with the thiol, c) oxidation, in $62 \%$ overall yield.

(26) Stridfeldt, E.; Seemann, A.; Bouma, M. J.; Dey, C.; Ertan, A.; Olofsson, B., Synthesis, Characterization and Unusual Reactivity of Vinylbenziodoxolones-Novel Hypervalent Iodine Reagents. Chem. Eur. J. 2016, 22, 16066-16070.

(27) Kraszkiewicz, L.; Skulski, L. Optimized Syntheses of Iodylarenes from Iodoarenes, with Sodium Periodate as the Oxidant. Part II. Arkivoc 2003, 2003, 120. 
(28) Helal, C. J.; Magriotis, P. A.; Corey, E. J. Direct Catalytic Enantioselective Reduction of Achiral $\alpha, \beta$-Ynones. Strong Remote Steric Effects Across the C-C Triple Bond. J. Am. Chem. Soc. 1996, 118, $10938-10939$.

(29) Brand, J. P.; Waser, J. Direct Alkynylation of Thiophenes: Cooperative Activation of TIPS-EBX with Gold and Brønsted Acids. Angew. Chem. Int. Ed. 2010, 49 (40), 7304-7307.

(30) Cuadrado, P.; González-Nogal, A. M.; Valero, R. Regiospecific Synthesis of 5-Silyl Azoles. Tetrahedron 2002, 58 (24), 4975-4980.

(31) Brand, J. P.; Chevalley, C.; Scopelliti, R.; Waser, J. Ethynyl Benziodoxolones for the Direct Alkynylation of Heterocycles: Structural Requirement, Improved Procedure for Pyrroles, and Insights into the Mechanism. Chem. Eur. J. 2012, 18 (18), 5655-5666.

(32) Boelke, A.; Caspers, L. D.; Nachtsheim, B. J. NH2-Directed C-H Alkenylation of 2-Vinylanilines with Vinylbenziodoxolones. Org. Lett. 2017, 19 (19), 5344-5347.

(33) Perozzi, E. F.; Michalak, R. S.; Figuly, G. D.; Stevenson, W. H.; Dess, D.; Ross, M. R.; Martin, J. C. Directed Dilithiation of Hexafluorocumyl Alcohol - Formation of a Reagent for the Facile Introduction of a Stabilizing Bidentate Ligand in Compounds of Hypervalent Sulfur (10-S-4), Phosphorus (10-P-5), Silicon (10-Si-5), and Iodine (10-I-3). J. Org. Chem. 1981, 46 (6), 1049-1053.

(34) Cvengroš, J.; Stolz, D.; Togni, A. A Concise Synthesis of Ortho-Iodobenzyl Alcohols via Addition of Ortho -Iodophenyl Grignard Reagent to Aldehydes and Ketones. Synthesis 2009, 2009 (16), 28182824.

(35) Blake, A. J.; Novak, A.; Davies, M.; Robinson, R. I.; Woodward, S. Preparation of 1,1'-OxyBis(3,3-Bis(Trifluoromethyl)-3(1H)-1,2-Benziodoxole) and 2-(N-(p-Toluenesulfonyl)Imino)Iodobenzylmethyl Ether. Synthetic Communications 2009, 39 (6), 1065-1075.

(36) Kitamura, T. An Alternative Synthesis of Alkynyl(Phenyl)Iodonium Triflates Using (Diacetoxyiodo)Benzene and Alkynylsilanes. Synthesis 1998, 1998 (10), 1416-1418.

(37) Kita, Y.; Shibata, N.; Tamura, O.; Miki, T. Chemistry of $O$-Silylated Ketene Acetals : A Mild and Convenient Synthesis of $\beta$-Lactam Antibiotics. Chem. Pharm. Bull. 1991, 39, 2225-2232. 
(38) Moghaddam, F. M.; Bardajee, G. R.; Chadorneshine Veranlou, R. O. KF/A12O3-Mediated Michael Addition of Thiols to Electron-Deficient Olefins. Synthetic Communications 2005, 35, 2427-2433. (39) Maitro, G.; Prestat, G.; Madec, D.; Poli, G. Preparation of Allyl Sulfoxides by Palladium-Catalyzed Allylic Alkylation of Sulfenate Anions. J. Org. Chem. 2006, 71, 7449-7454.

(40) Yue, H.-L.; Klussmann, M. Acid-Catalyzed Oxidative Addition of Thiols to Olefins and Alkynes for a One-Pot Entry to Sulfoxides. Synlett 2016, 27, 2505-2509.

(41) Cui, H.; Wei, W.; Yang, D.; Zhang, Y.; Zhao, H.; Wang, L.; Wang, H. Visible-Light-Induced Selective Synthesis of Sulfoxides from Alkenes and Thiols Using Air as the Oxidant. Green Chem. 2017, $19,3520-3524$.

(42) López, R.; Zalacain, M.; Palomo, C. Catalytic Asymmetric Synthesis of $\gamma$-Substituted Vinyl Sulfones. Chem. Eur. J. 2011, 17 (8), 2450-2457.

(43) Shinde, P. S.; Patil, N. T. Gold-Catalyzed Dehydrazinative C(Sp)-S Coupling Reactions of Arylsulfonyl Hydrazides with Ethynylbenziodoxolones for Accessing Alkynyl Sulfones. Eur. J. Org. Chem. 2017, 2017, 3512-3515. 
Supporting Information for

\title{
Metal-Free Electrophilic Alkynylation of Sulfenate Anions with Ethynylbenziodoxolone Reagents
}

\author{
Stephanie G. E. Amos, Stefano Nicolai, Alec Gagnebin, Franck Le Vaillant and Jerome Waser * \\ Laboratory of Catalysis and Organic Synthesis, Ecole Polytechnique Fédérale de Lausanne, \\ EPFL SB ISIC LCSO, BCH 4306, 1015 Lausanne, Switzerland \\ jerome.waser@epfl.ch
}

(65 pages) 


\section{$\underline{\text { Table of Contents }}$}

1. Optimisation of sulfoxide alkynylation reactions conditions ...........................................S3

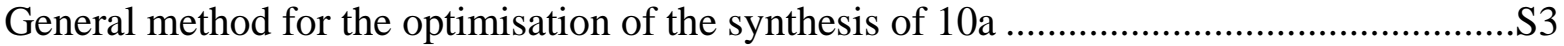

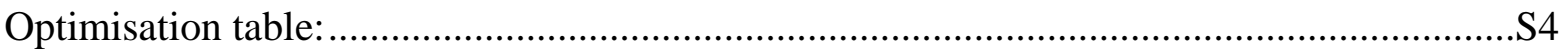

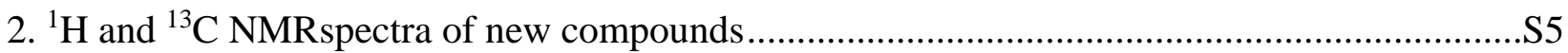




\section{Optimisation of sulfoxide alkynylation reactions conditions}

\section{General method for the optimisation of the synthesis of $10 \mathrm{a}$}

Into an oven-dried test-tube equipped with a magnetic stirrer, tert-Butyl 3-(4methylphenylsulfinyl)propanoate $(\mathbf{6 a}, \mathrm{X} \mathrm{mmol})$ and the hypervalent iodine reagent $(\mathbf{9}, \mathrm{Y} \mathrm{mmol})$ anhydrous toluene ( $2 \mathrm{~mL}, 0.05 \mathrm{M}$, unless specified otherwise) and the base ( $\mathrm{Z}$ equiv.) were then added and the reaction mixture was stirred at $-40{ }^{\circ} \mathrm{C}$, unless specified otherwise. The crude mixture was then filtered over $\mathrm{SiO}_{2}$. The resulting solution was concentrated under reduced pressure. ${ }^{1} \mathrm{H}$ NMR yield was determined using $\mathrm{CH}_{2} \mathrm{Br}_{2}$ as internal standard: $\mathrm{CH}_{2} \mathrm{Br}_{2}(7 \mu \mathrm{L}, 0.1 \mathrm{mmol})$ was added to the crude and the crude was homogenised with Chloroform- $d$. The crude product was then purified by preparative TLC ( $\mathrm{SiO}_{2}$ glass plate, Heptane/EtOAc: 8/2). Compound was then retrieved using EtOAc.

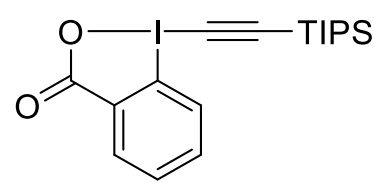

$9 a$

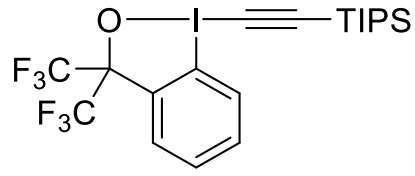

25

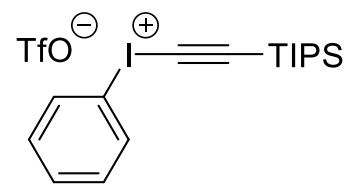

26

Figure S1: Reagents tested during optimisation 
Table S1: Optimisation of the alkynylation reaction

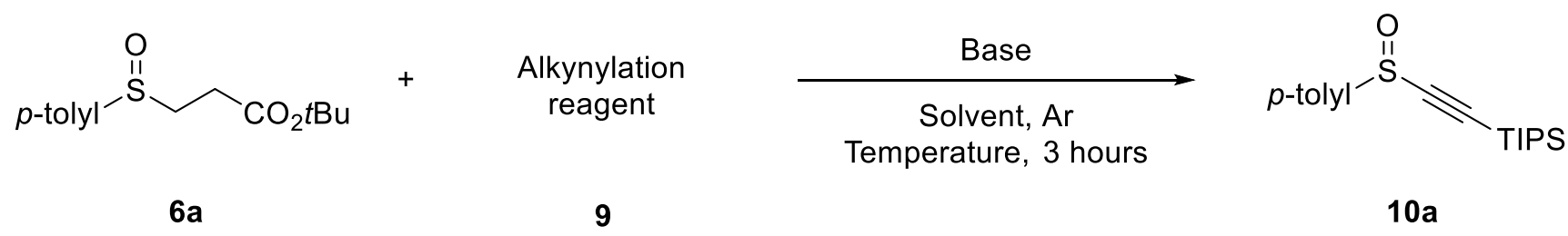

\begin{tabular}{|c|c|c|c|c|c|c|c|}
\hline Entry & T. $\left({ }^{\circ} \mathbf{C}\right)$ & X (mmol) & Reagent & $\mathrm{Y}(\mathrm{mmol})$ & Base ( $\mathrm{Z}$ equiv.) & Solvent & Yield (\%) \\
\hline 1 & -78 & 0.1 & $9 \mathbf{a}$ & 0.11 & $\mathrm{KO} t \mathrm{Bu}(1.05)$ & $\operatorname{PhMe}(0.16 \mathrm{M})$ & 64 \\
\hline 2 & -40 & 0.1 & $9 a$ & 0.11 & $\mathrm{KO} t \mathrm{Bu}(1.05)$ & $\mathrm{PhMe}(0.16 \mathrm{M})$ & $70(77)$ \\
\hline 3 & -25 & 0.1 & $9 a$ & 0.11 & $\mathrm{KO} t \mathrm{Bu}(1.05)$ & $\mathrm{PhMe}(0.16 \mathrm{M})$ & 68 \\
\hline 4 & -40 & 0.1 & $9 a$ & 0.11 & $\mathrm{NaO} t \mathrm{Bu}(1.05)$ & $\mathrm{PhMe}$ & (53) \\
\hline 5 & -40 & 0.1 & $9 \mathbf{a}$ & 0.11 & KHDMS (1.05) & $\mathrm{PhMe}$ & (70) \\
\hline 6 & -40 & 0.1 & $9 \mathbf{a}$ & 0.11 & LDA & $\mathrm{PhMe}$ & (52) \\
\hline 7 & -40 & 0.1 & 25 & 0.11 & $\mathrm{KO} t \mathrm{Bu}(1.05)$ & $\mathrm{PhMe}$ & (84) \\
\hline 8 & -40 & 0.1 & 26 & 0.11 & $\mathrm{KO} t \mathrm{Bu}(1.05)$ & $\mathrm{PhMe}$ & (44) \\
\hline 9 & -40 & 0.11 & $9 \mathbf{a}$ & 0.1 & $\mathrm{KO} t \mathrm{Bu}(1.05)$ & $\mathrm{PhMe}$ & 75 \\
\hline 10 & -40 & 0.1 & $9 \mathbf{a}$ & 0.11 & $\mathrm{KO} t \mathrm{Bu}(1.05)$ & DMF & 28 \\
\hline 11 & -40 & 0.1 & $9 a$ & 0.11 & $\mathrm{KO} t \mathrm{Bu}(1.05)$ & PhMe:DCM (7:3) & (58) \\
\hline 12 & -40 & 0.1 & 9a & 0.11 & $\mathrm{KO} t \mathrm{Bu}(1.05)$ & PhMe:DCM (9:1) & (66) \\
\hline 13 & -40 & 0.1 & 9a & 0.11 & $\mathrm{KO} t \mathrm{Bu}(1.05)$ & THF & (66) \\
\hline $14^{a}$ & -40 & 0.1 & $9 a$ & 0.11 & $\mathrm{KO} t \mathrm{Bu}(1.05)$ & PhMe & 81 \\
\hline $15^{\mathrm{a}}$ & -40 & 0.1 & $9 a$ & 0.11 & $\mathrm{KO} t \mathrm{Bu}(1.25)$ & $\mathrm{PhMe}$ & 60 \\
\hline $16^{\mathrm{a}}$ & -40 & 0.11 & $9 a$ & 0.1 & $\mathrm{KO} t \mathrm{Bu}(1.05)$ & $\mathrm{PhMe}^{\mathrm{b}}$ & 90 \\
\hline $17^{\mathrm{a}, \mathrm{c}}$ & RT & 0.11 & $9 \mathbf{a}$ & 0.1 & $\mathrm{KO} t \mathrm{Bu}(1.05)$ & $\mathrm{PhMe}^{\mathrm{d}}$ & 73 \\
\hline
\end{tabular}

${ }^{a}$ Oxygen free procedure: 6a and 9a were introduced into an oven-dried flask equipped with a magnetic stirrer. After three vaccum/ $\mathrm{N}_{2}$ cycles potassium tert-butoxide was added under the inert atmosphere of a glove-box. ${ }^{b}$ Degassed with a ballon of argon while cooling down to $-40{ }^{\circ} \mathrm{C}$. ${ }^{\mathrm{c}} \mathrm{Two}$ impurities were identified as tert-butyl sulfinate (S1) and alkynyl sulfone (S2) Degassed with a ballon of argon at RT.

Tert-butyl 4-methylbenzenesulfinate (S1):

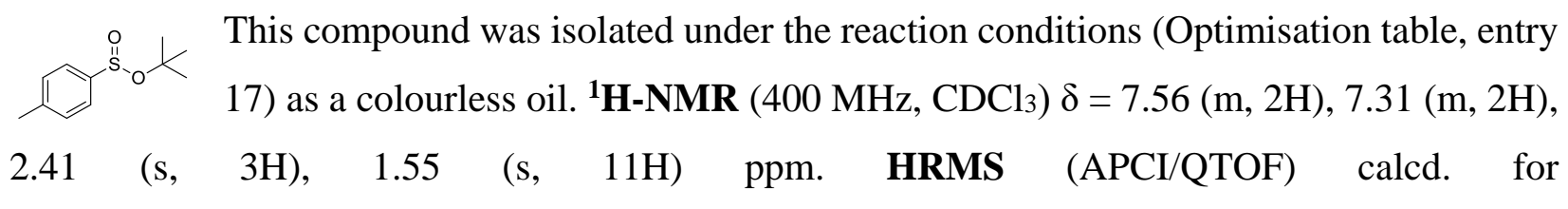


$\mathrm{C}_{11} \mathrm{H}_{16} \mathrm{NaO}_{2} \mathrm{~S}^{+}[\mathrm{M}+\mathrm{Na}]^{+} 235.0763$; found 235.0759. The values of the NMR spectra are in accordance with reported literature data. ${ }^{1}$

Triisopropyl(tosylethynyl)silane (S2):

This compound was isolated under the reaction conditions (Optimisation table, $(\mathrm{m}, 2 \mathrm{H}), 2.46(\mathrm{~s}, 3 \mathrm{H}), 1.16-0.92(\mathrm{~m}, 21 \mathrm{H}) \mathrm{ppm}$. HRMS (ESI/QTOF) m/z: calcd. for $\mathrm{C}_{18} \mathrm{H}_{29} \mathrm{O}_{2} \mathrm{SSi}^{+}[\mathrm{M}+\mathrm{H}]^{+} 337.1652$; found 337.1657. The values of the NMR spectra are in accordance with reported literature data. ${ }^{2}$

\section{2. ${ }^{1} \mathrm{H}$ and ${ }^{13} \mathrm{C}$ NMRspectra of new compounds}

1 Kadari, L.; Radha Krishna, P.; Lakshmi Prapurna, Y. Sulfination of Alcohols with PToluenesulfonylmethyl Isocyanide under Metal-Free Conditions: A Mitsunobu Approach. Adv. Syn. Catal. 2016, 358, 3863-3868.

${ }^{2}$ Shinde, P. S.; Patil, N. T. Gold-Catalyzed Dehydrazinative C(Sp)-S Coupling Reactions of Arylsulfonyl Hydrazides with Ethynylbenziodoxolones for Accessing Alkynyl Sulfones. Eur. J. Org. Chem. 2017, 2017, 3512-3515. 
Methyl 3-((4-methylphenyl)sulfinyl)propanoate (5)

${ }^{1} \mathrm{H}$ NMR (400 MHz, Chloroform-d)

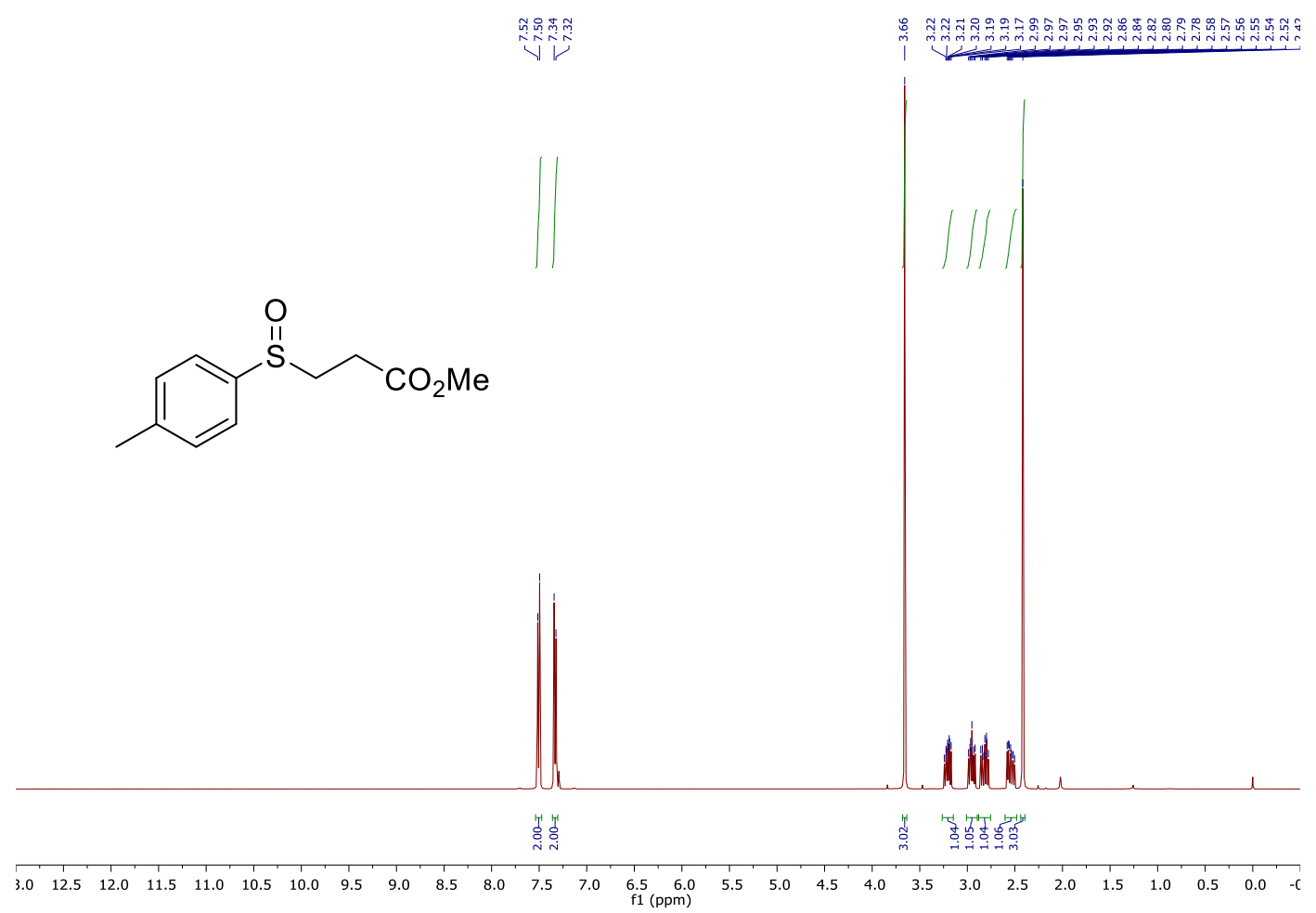


1-Methyl-4-[(2-nitroethyl)sulfinyl]benzene (8)

${ }^{1} \mathrm{H}$ NMR (400 MHz, Chloroform- $d$ )

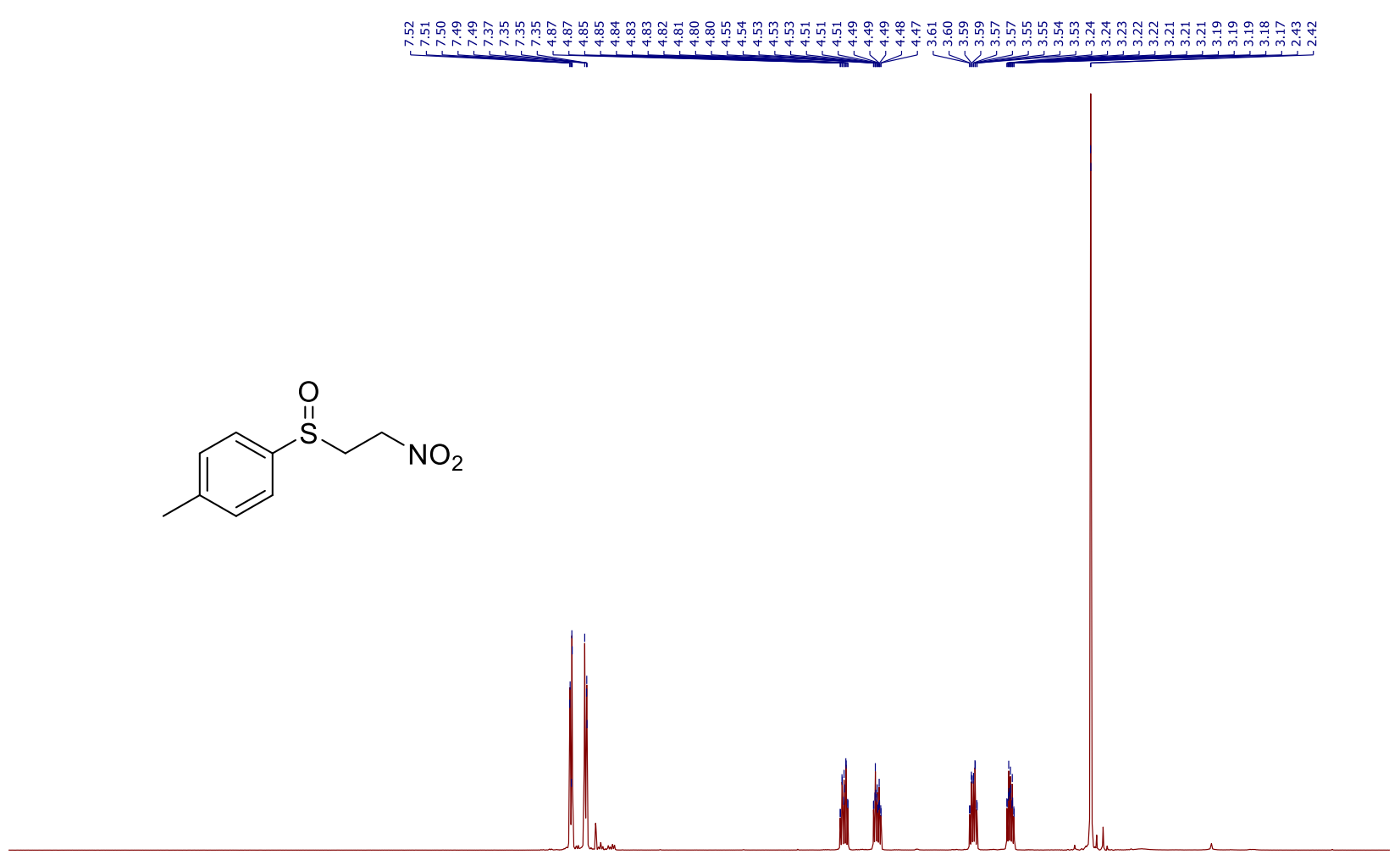

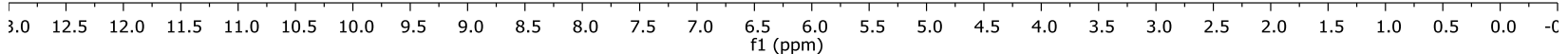




\section{(E)-1-Styryl-1 $\lambda^{3}$-benzo $[d][1,2]$ iodaoxol-3(1H)-one (11a)}

${ }^{1} \mathrm{H}$ NMR (400 MHz, Chloroform- $d$ )

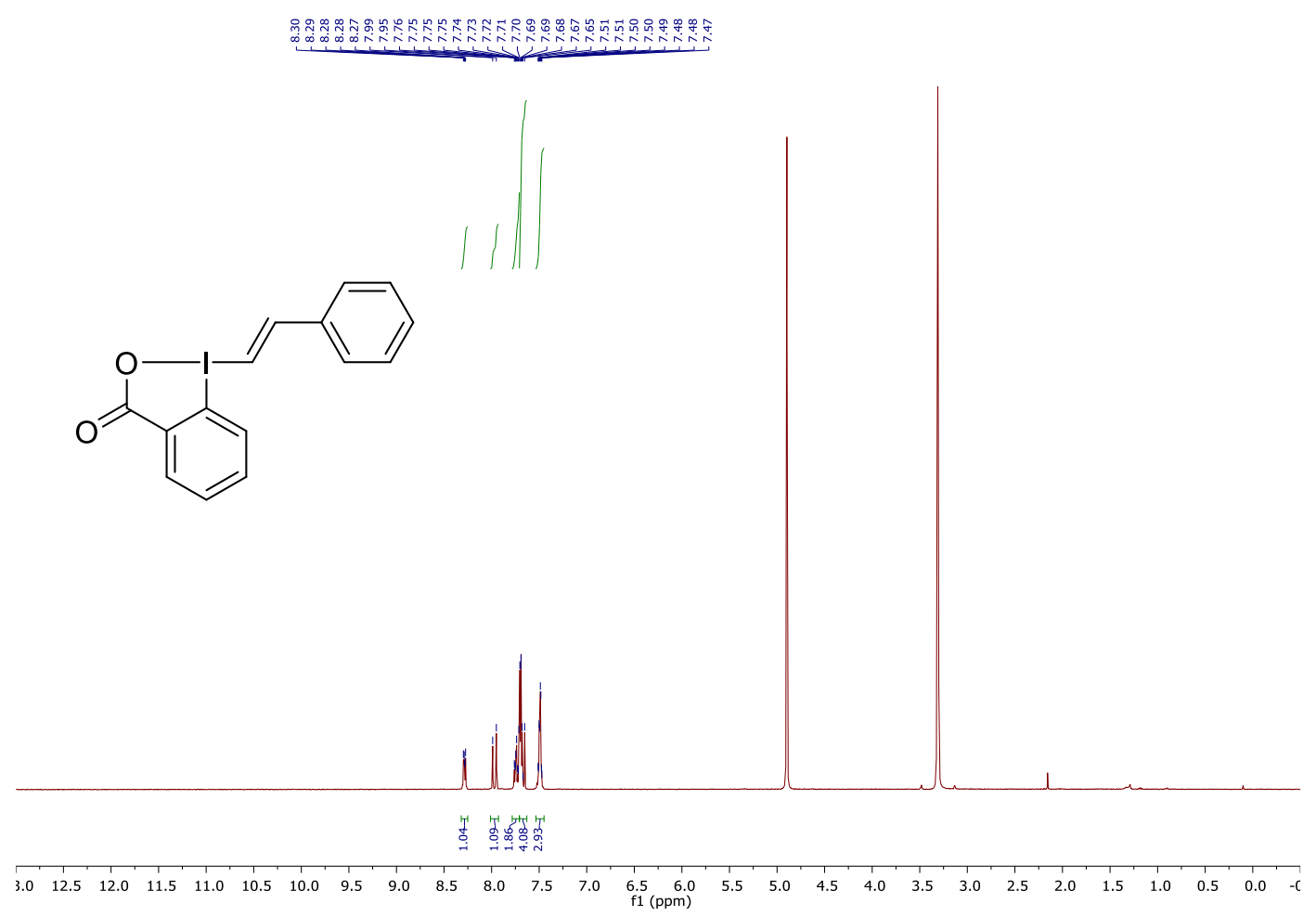




\section{(E)-1-(4-Methylstyryl)-1 $\lambda^{3}$-benzo[d][1,2]iodaoxol-3(1H)-one (11b)}

${ }^{1} \mathrm{H}$ NMR (400 MHz, Chloroform-d)

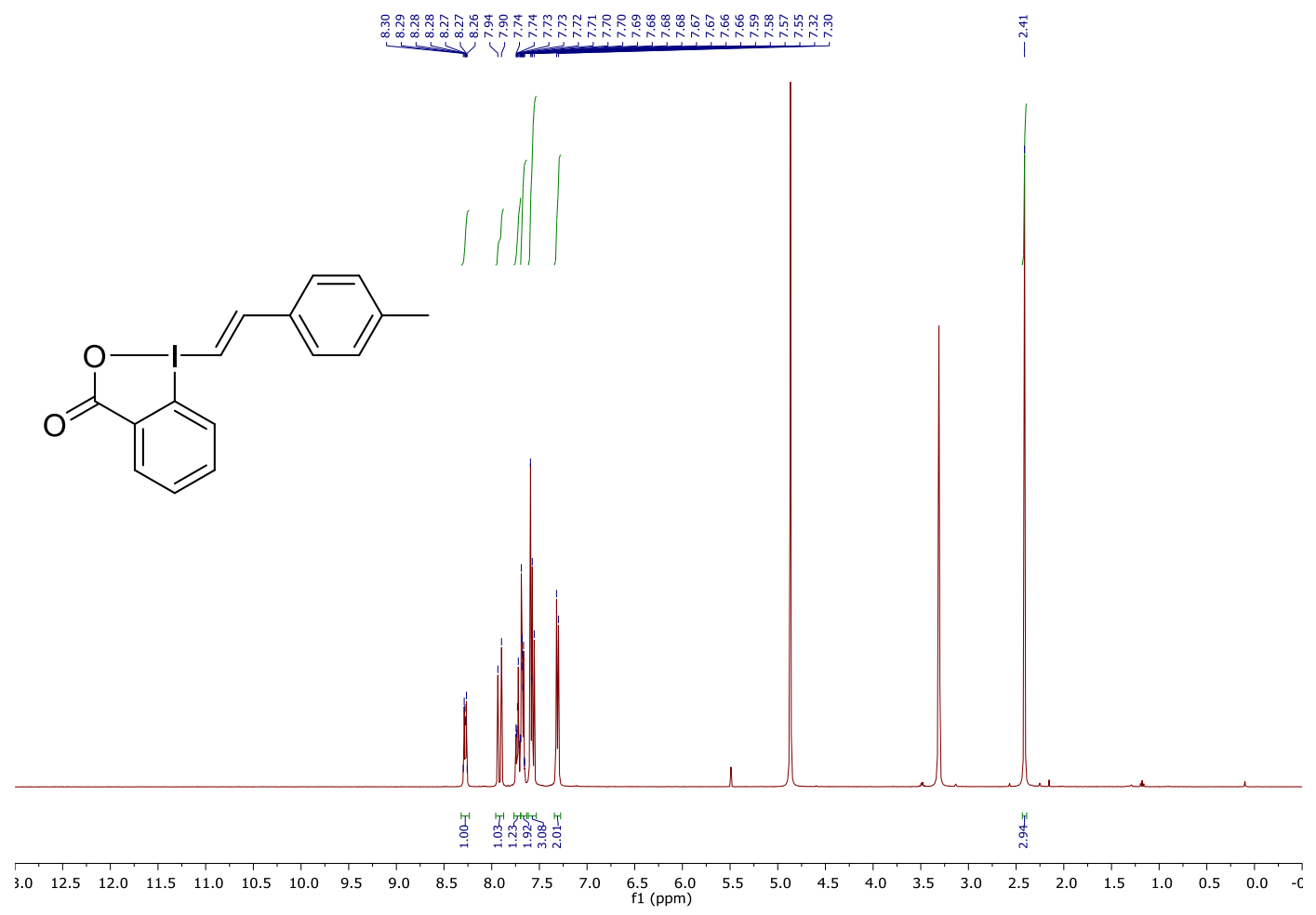




\section{Tert-butyl 3-(4-methylphenylthio)propanoate (21a)}

${ }^{1} \mathrm{H}$ NMR (400 MHz, Chloroform-d)

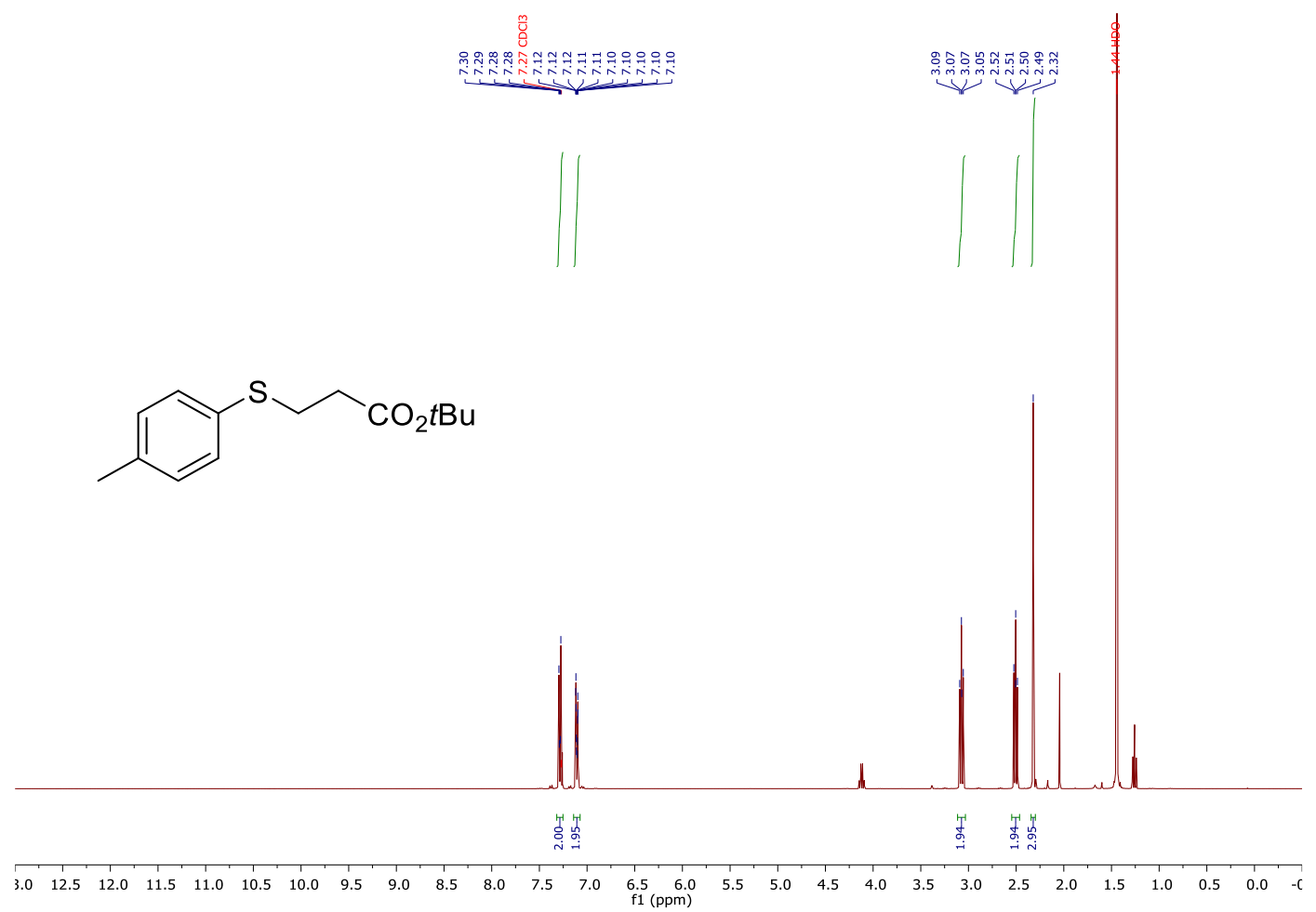




\section{Tert-butyl 3-(phenylthio)propanoate (21b)}

${ }^{1} \mathrm{H}$ NMR (400 MHz, Chloroform- $d$ )

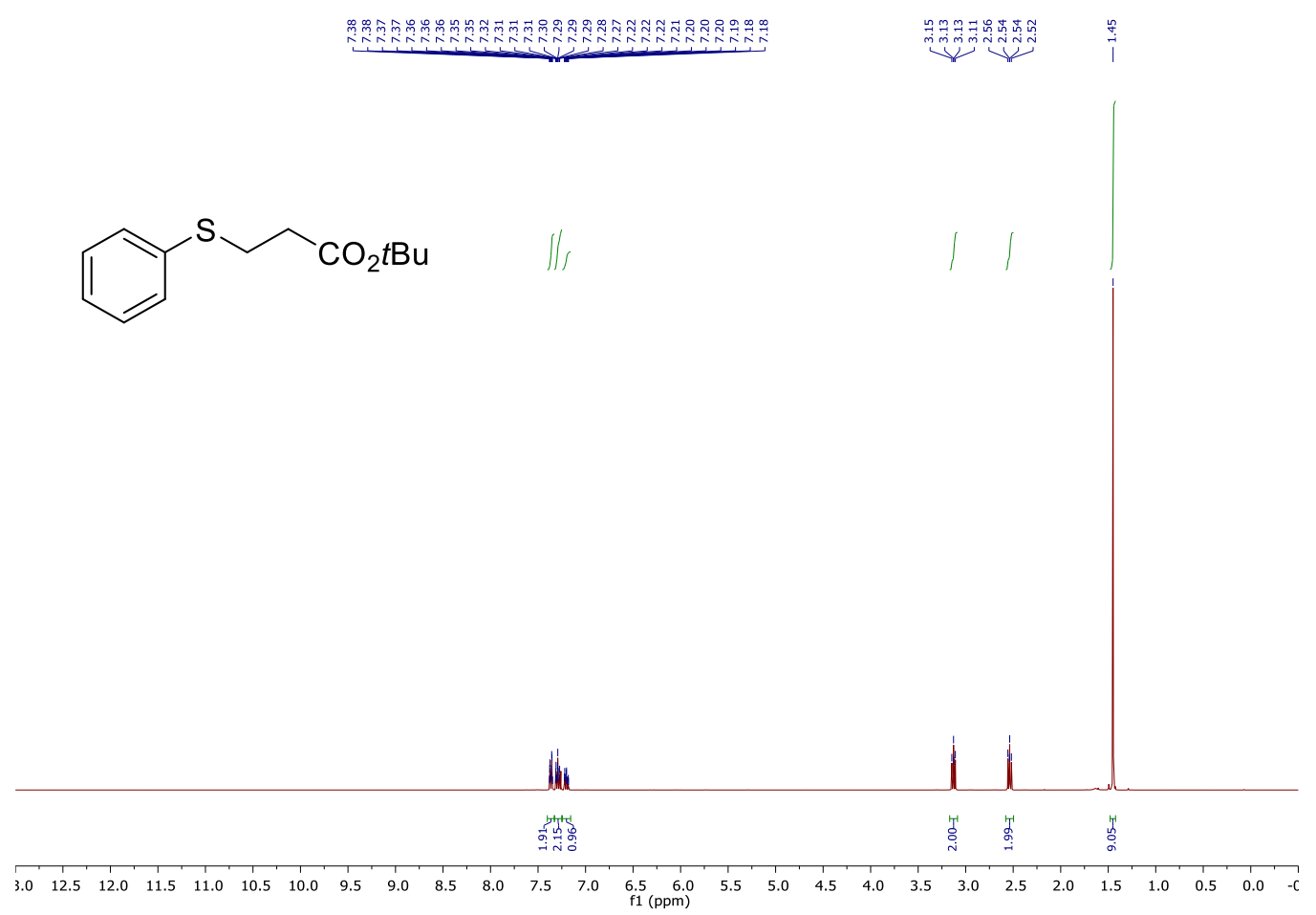

${ }^{13} \mathrm{C}$ NMR (101 MHz, Chloroform- $d$ )

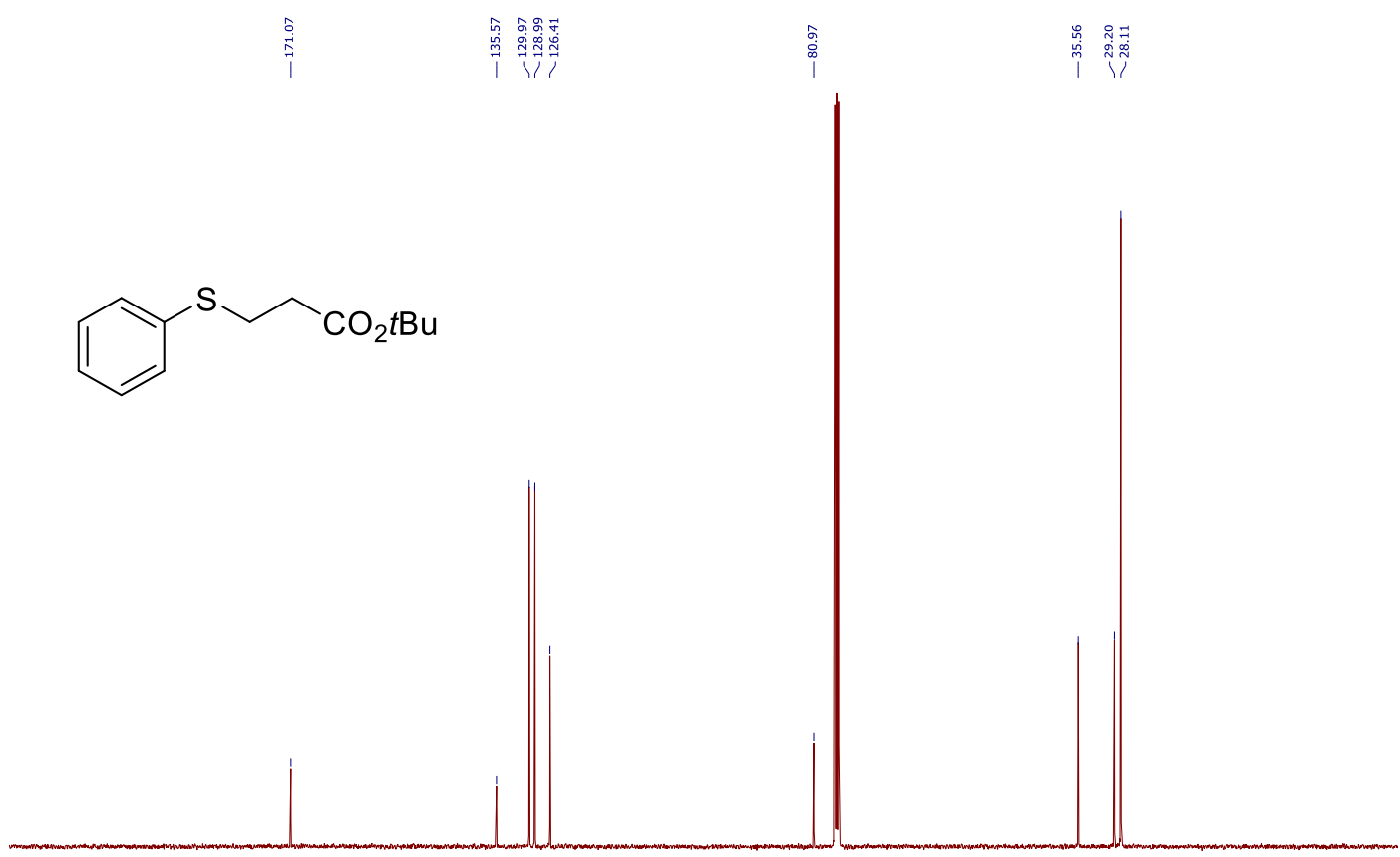

$\begin{array}{lllllllllllllllllllllllllllllll}210 & 200 & 190 & 180 & 170 & 160 & 150 & 140 & 130 & 120 & 110 & 100 & 90 & 80 & 70 & 60 & 50 & 40 & 30 & 20 & 10 & 0 & -10\end{array}$ 


\section{Tert-butyl 3-(4-methoxyphenylthio)propanoate (21c)}

${ }^{1} \mathrm{H}$ NMR (400 MHz, Chloroform- $d$ )

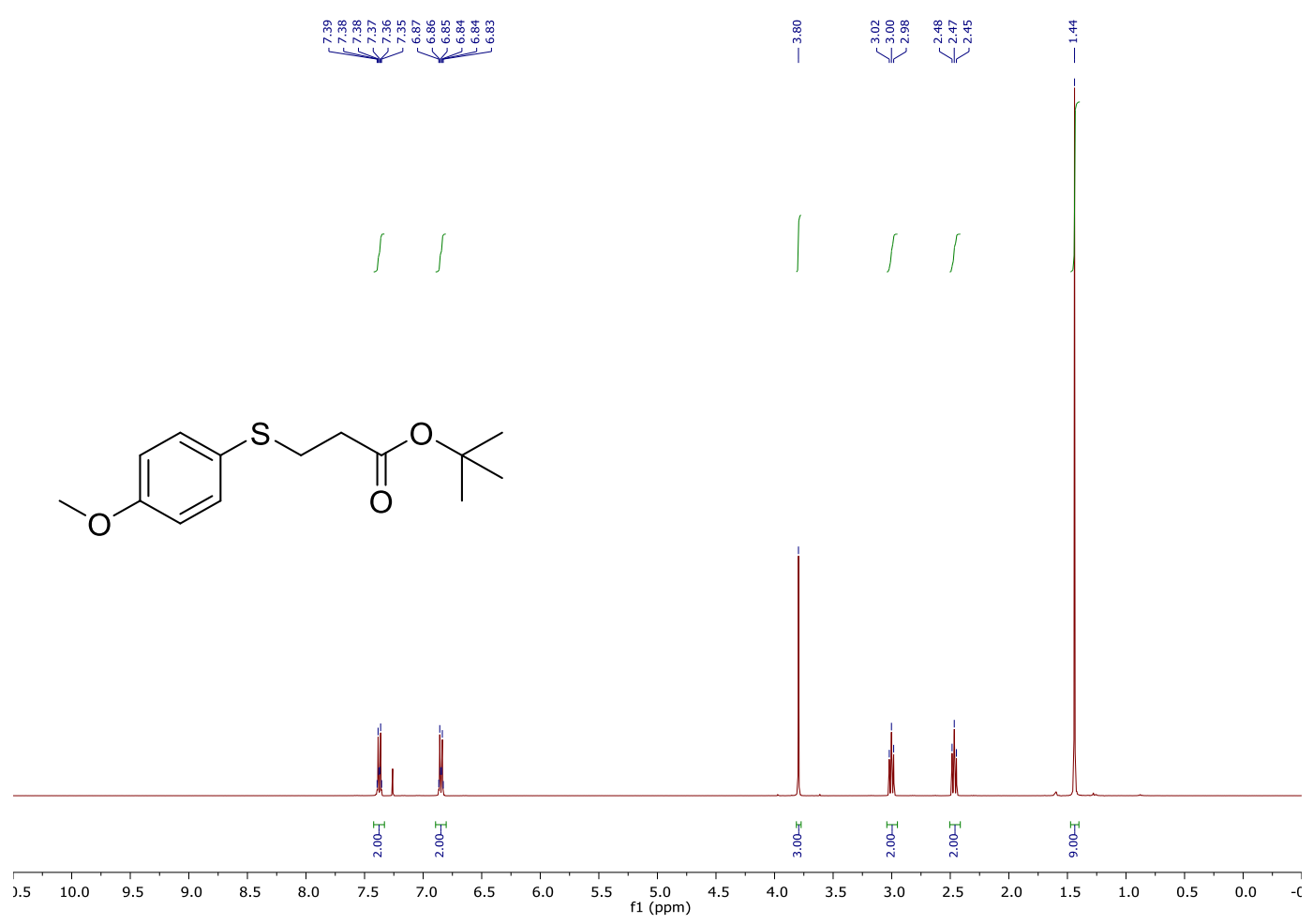

${ }^{13} \mathrm{C}$ NMR (101 MHz, Chloroform- $d$ )
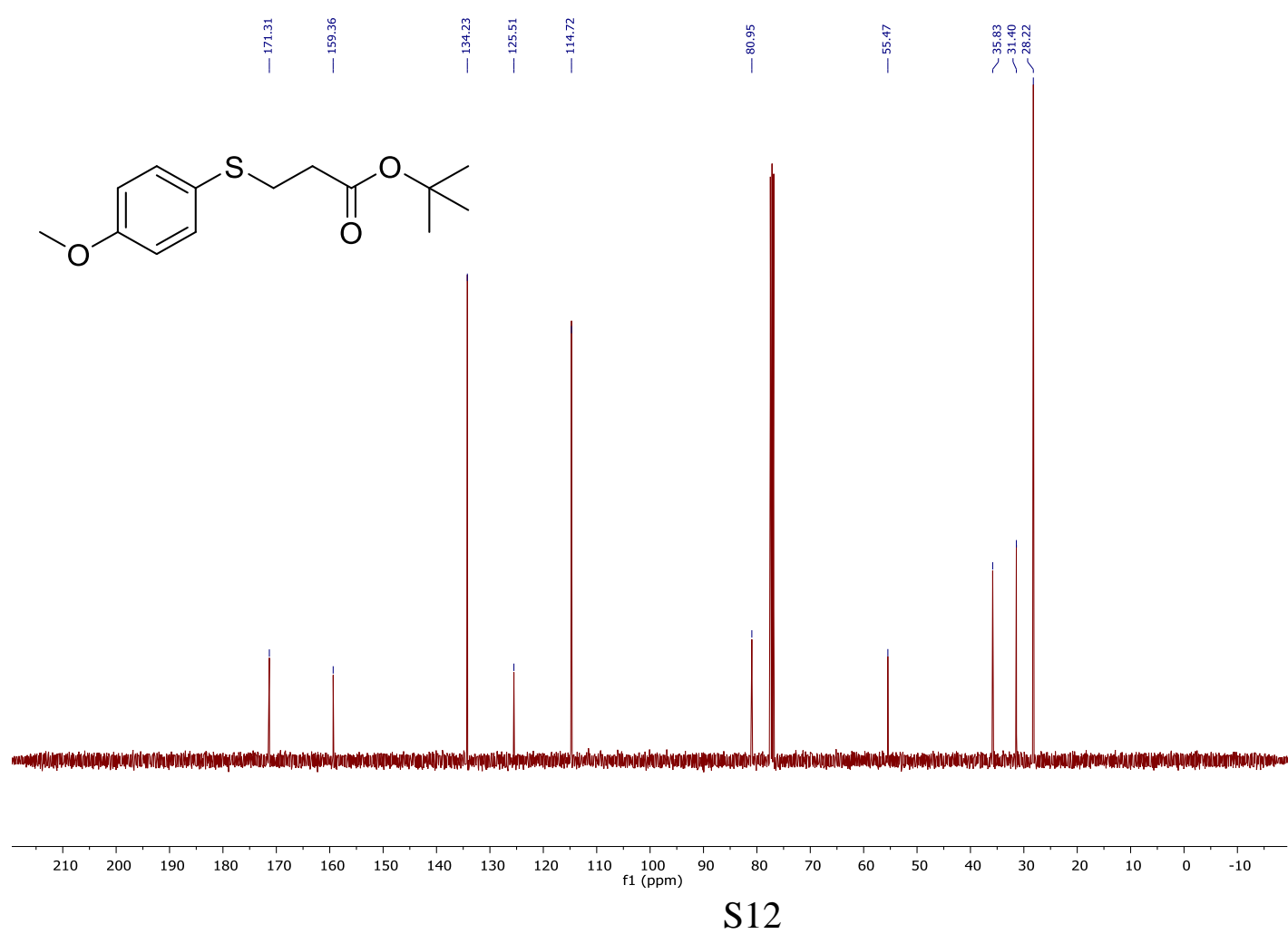


\section{Tert-butyl 3-(4-bromophenylthio)propanoate (21d)}

${ }^{1} \mathrm{H}$ NMR (400 MHz, Chloroform- $d$ )

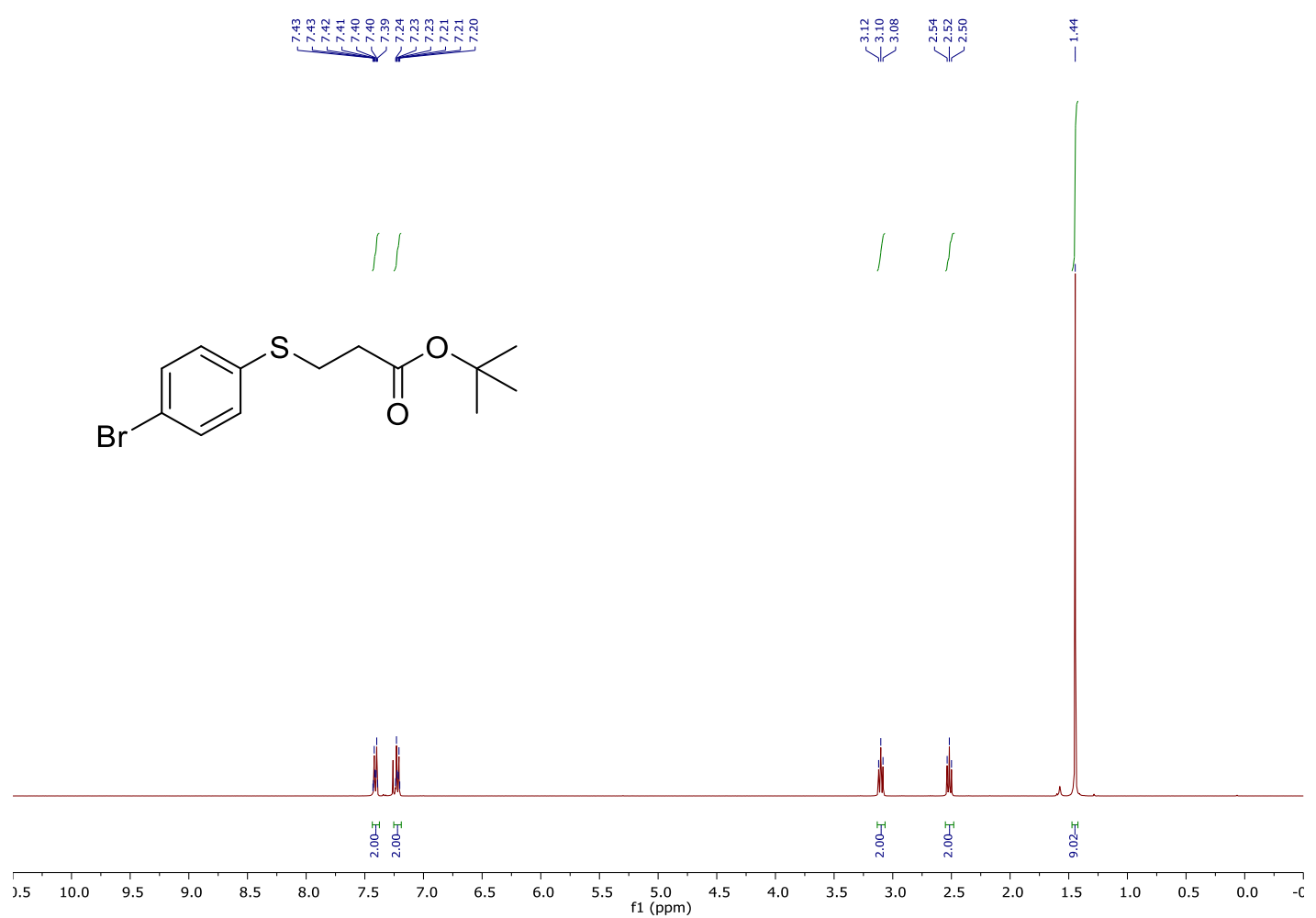

${ }^{13} \mathrm{C}$ NMR (101 MHz, Chloroform- $d$ )

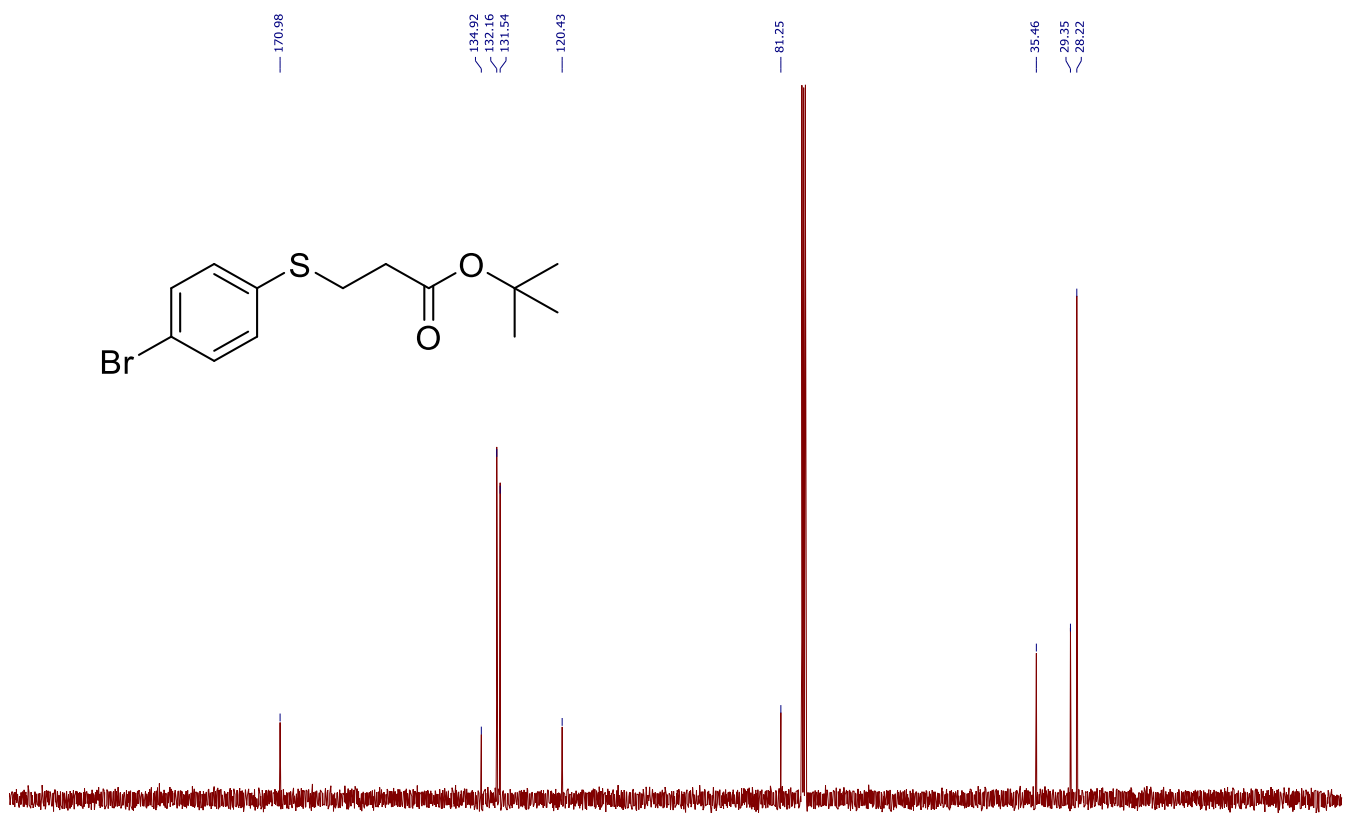

$\begin{array}{lllllllllllllllllllllllllllllllllllllllll}210 & 200 & 190 & 180 & 170 & 160 & 150 & 140 & 130 & 120 & 110 & 100 & 90 & 80 & 70 & 60 & 50 & 40 & 30 & 20 & 10 & 0 & -10\end{array}$ 


\section{Tert-butyl 3-(4-fluorophenylthio)propanoate (21e)}

${ }^{1} \mathrm{H}$ NMR (400 MHz, Chloroform- $d$ )

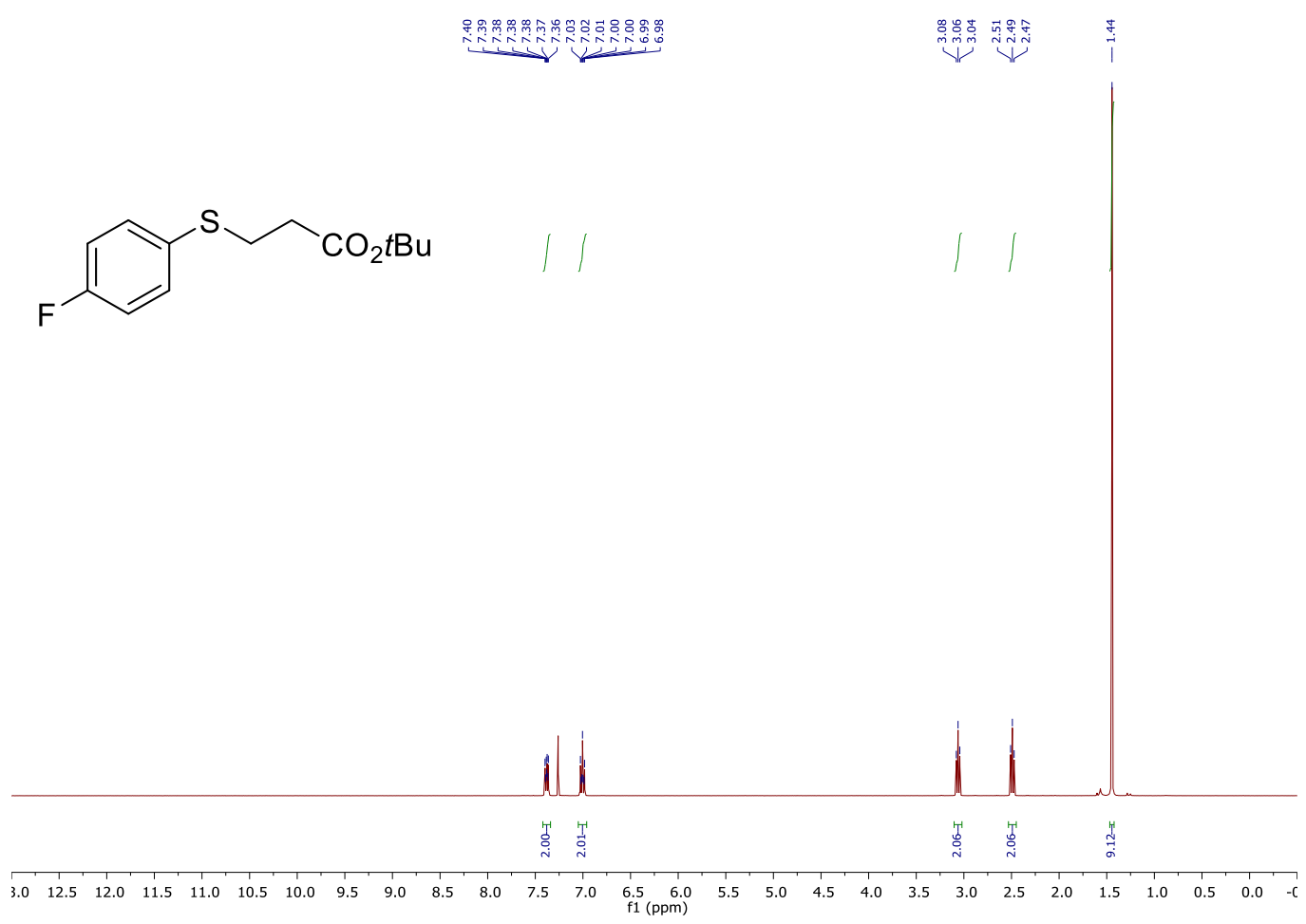

${ }^{13} \mathrm{C}$ NMR (101 MHz, Chloroform- $d$ )

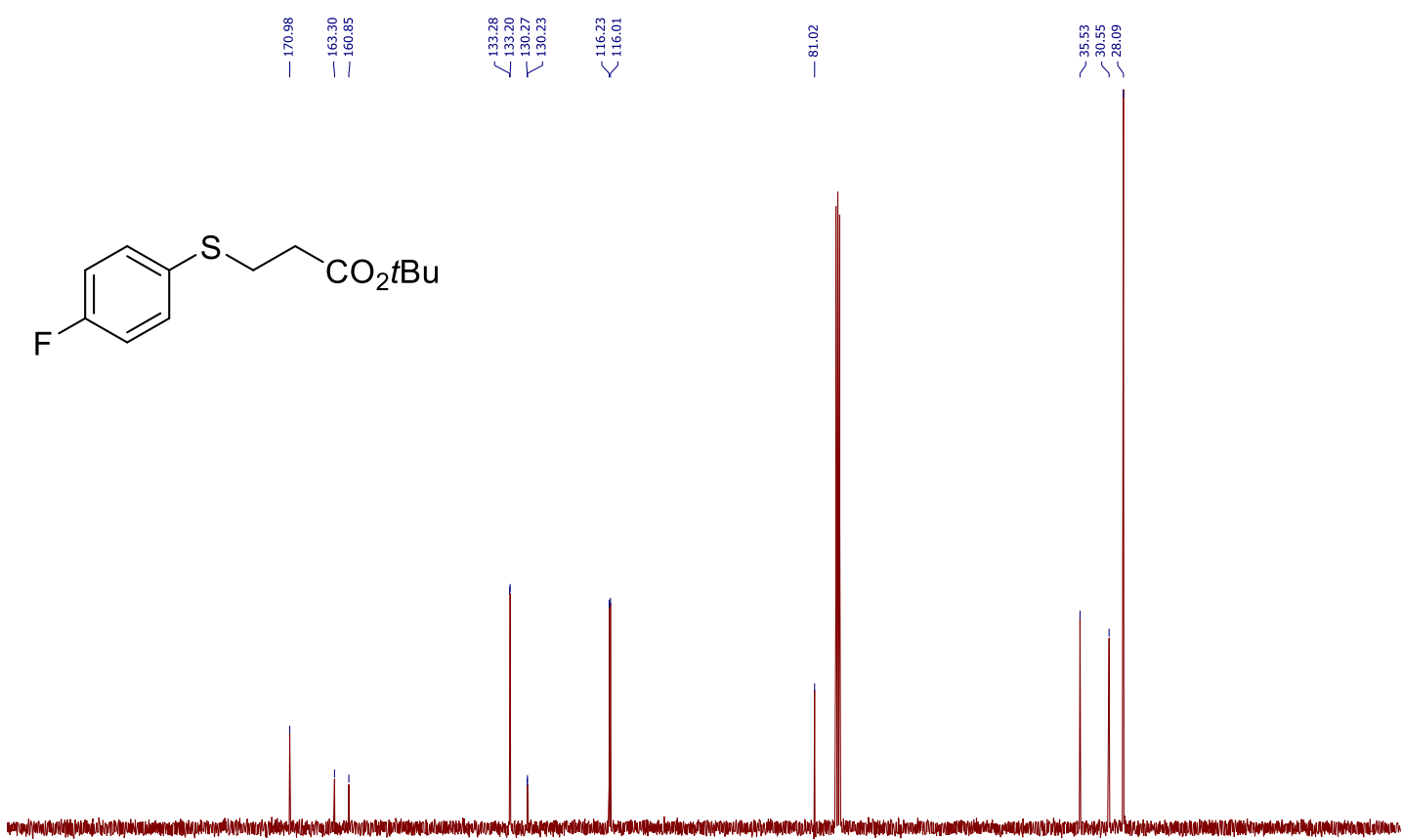

$\begin{array}{llllllllllllllllllllllll}210 & 200 & 190 & 180 & 170 & 160 & 150 & 140 & 130 & 120 & 110 & \begin{array}{c}100 \\ \mathrm{f} 1(\mathrm{ppm})\end{array} & 90 & 80 & 70 & 60 & 50 & 40 & 30 & 20 & 10 & 0 & -10\end{array}$ 
${ }^{19}$ F NMR (376 MHz, Chloroform-d)

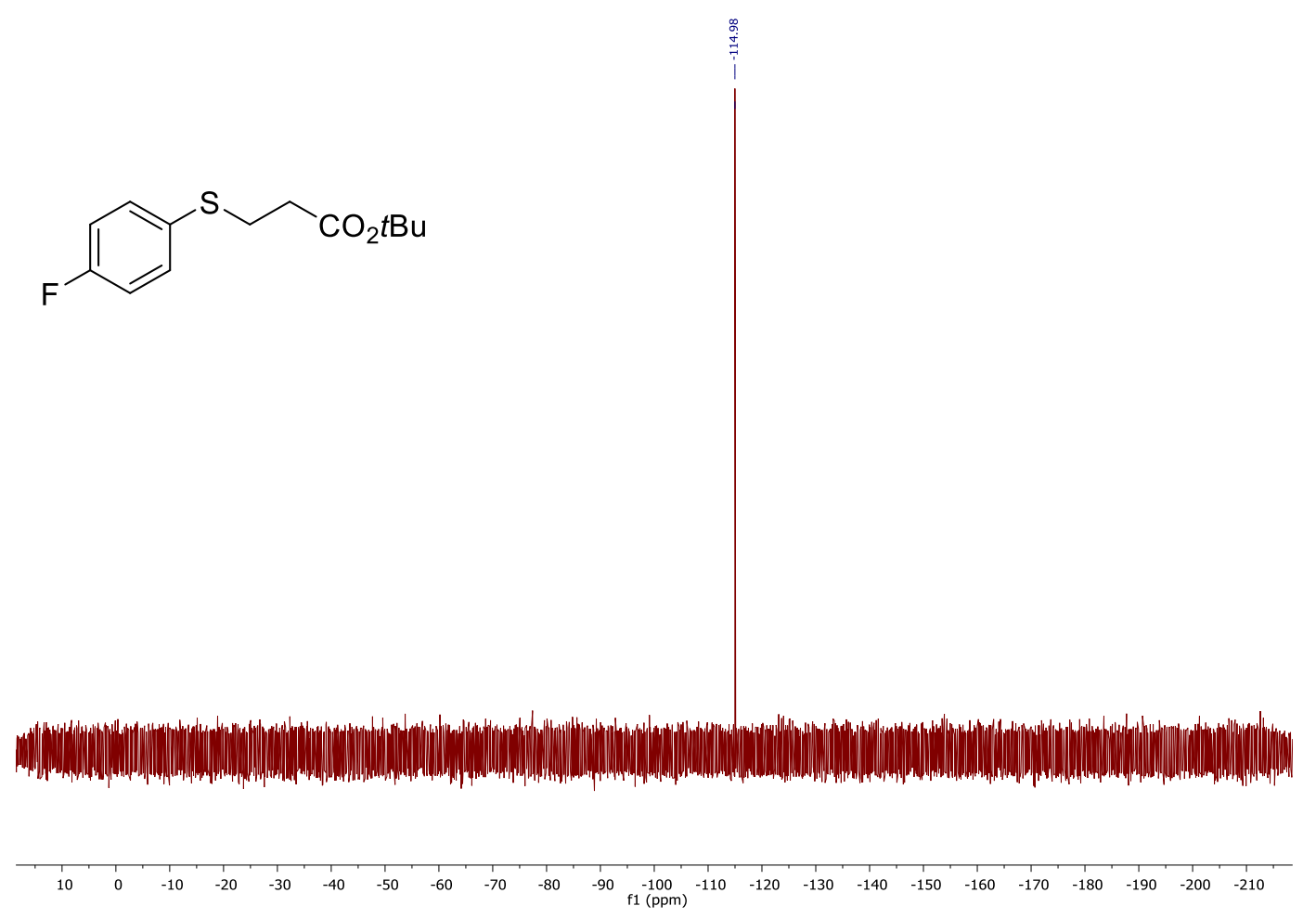




\section{Tert-butyl 3-(4-trifluoromethylphenylthio)propanoate (21f)}

${ }^{1} \mathrm{H}$ NMR (400 MHz, Chloroform- $d$ )

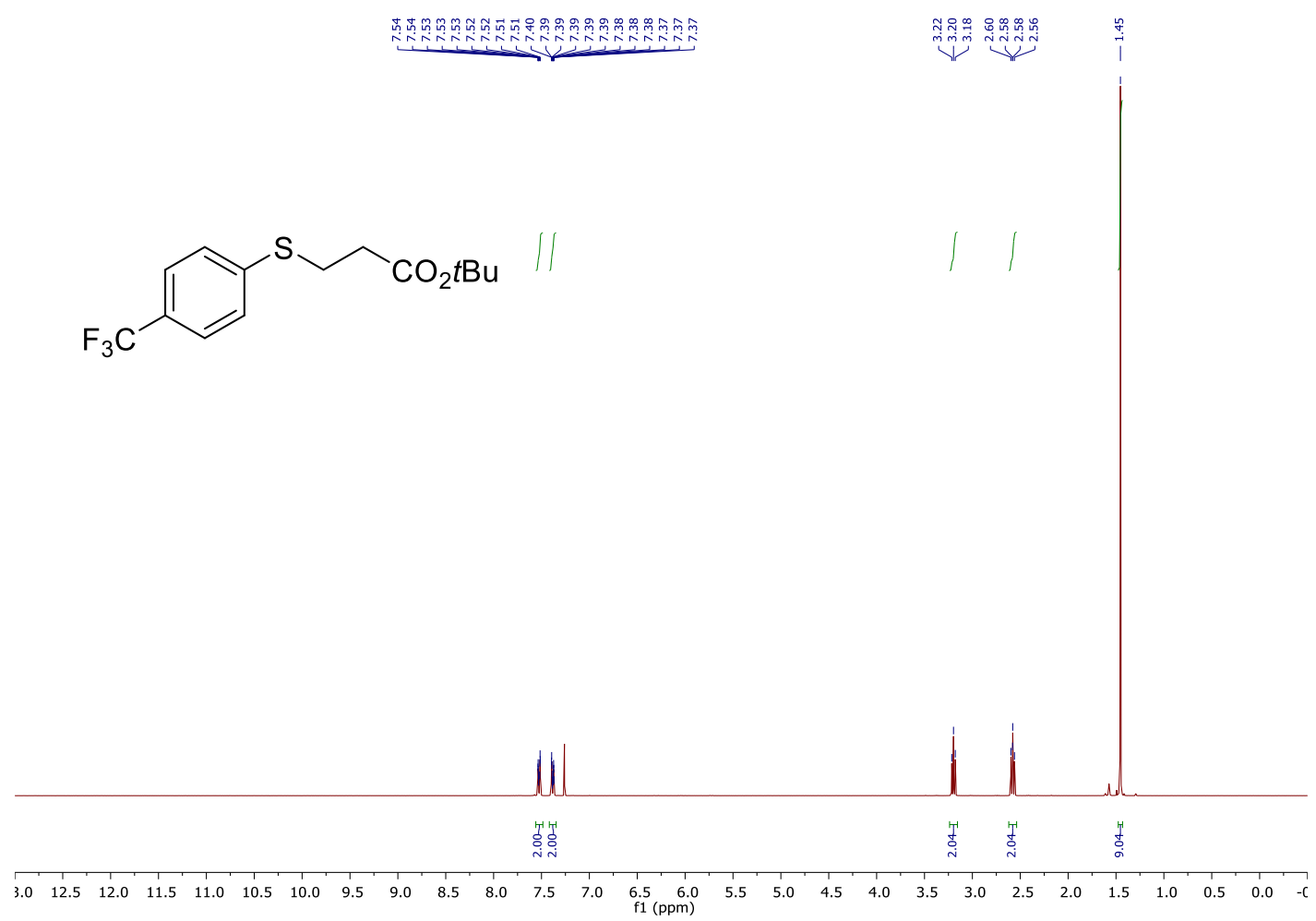

${ }^{13} \mathrm{C}$ NMR (101 MHz, Chloroform- $d$ )
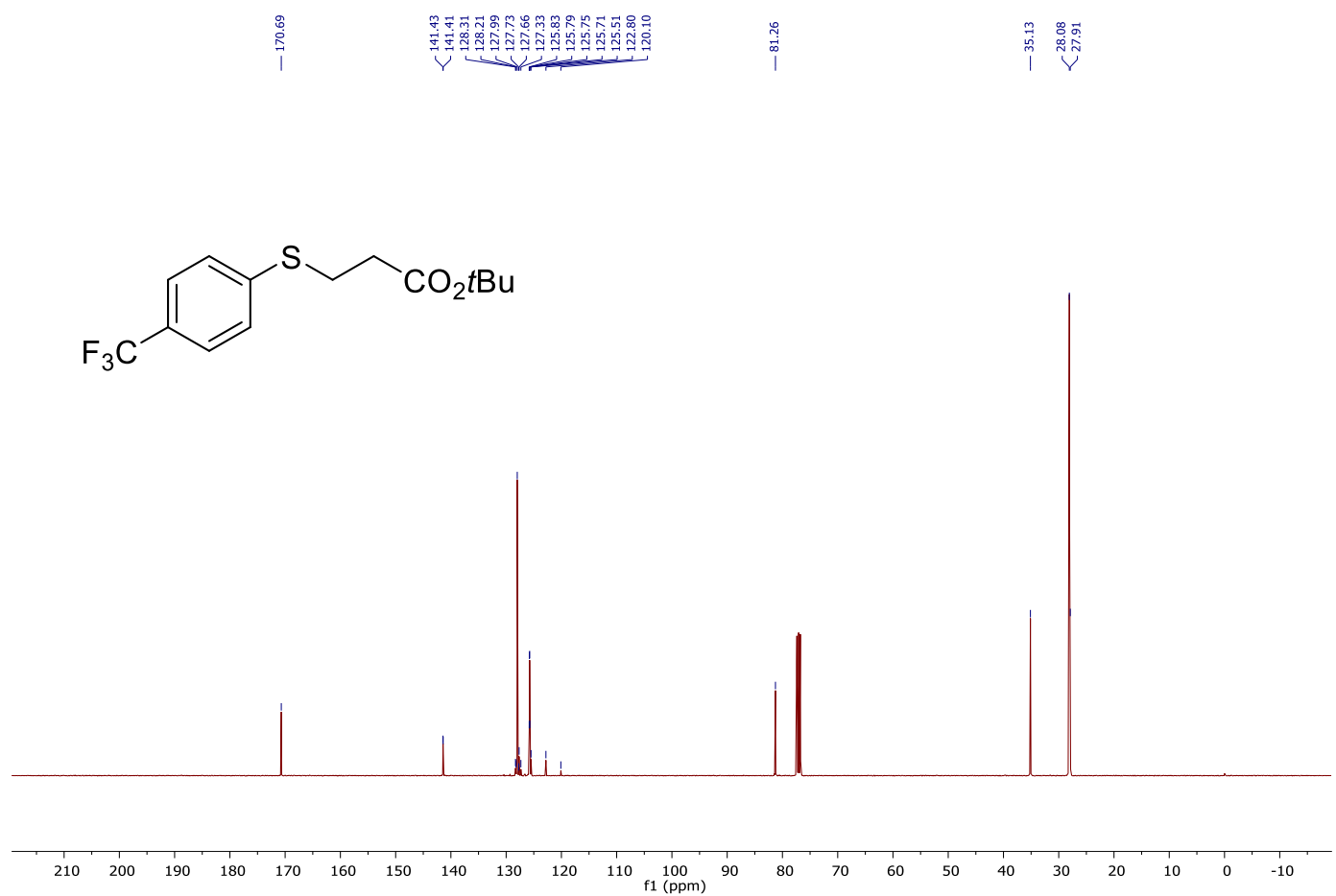
${ }^{19}$ F NMR (376 MHz, Chloroform-d)

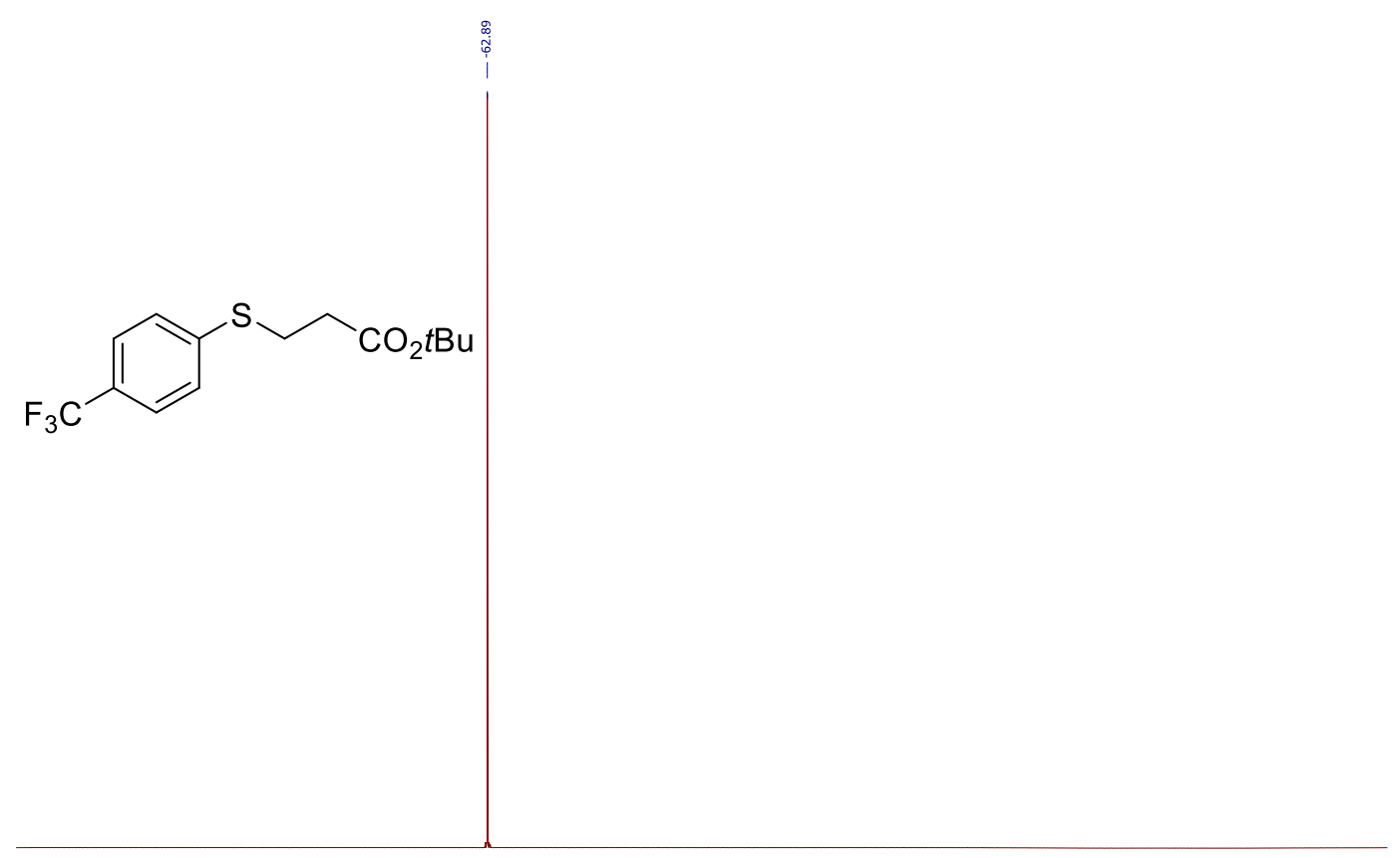

$\begin{array}{lllllllllllllllllllllllll}1 & 0 & 0 & -10 & -20 & -30 & -40 & -50 & -60 & -70 & -80 & -90 & -100 & -110 & -120 & -130 & -140 & -150 & -160 & -170 & -180 & -190 & -200 & -210\end{array}$ 


\section{Tert-butyl 3-(4-nitrophenylthio)propanoate (21g)}

${ }^{1} \mathrm{H}$ NMR (400 MHz, Chloroform- $d$ )

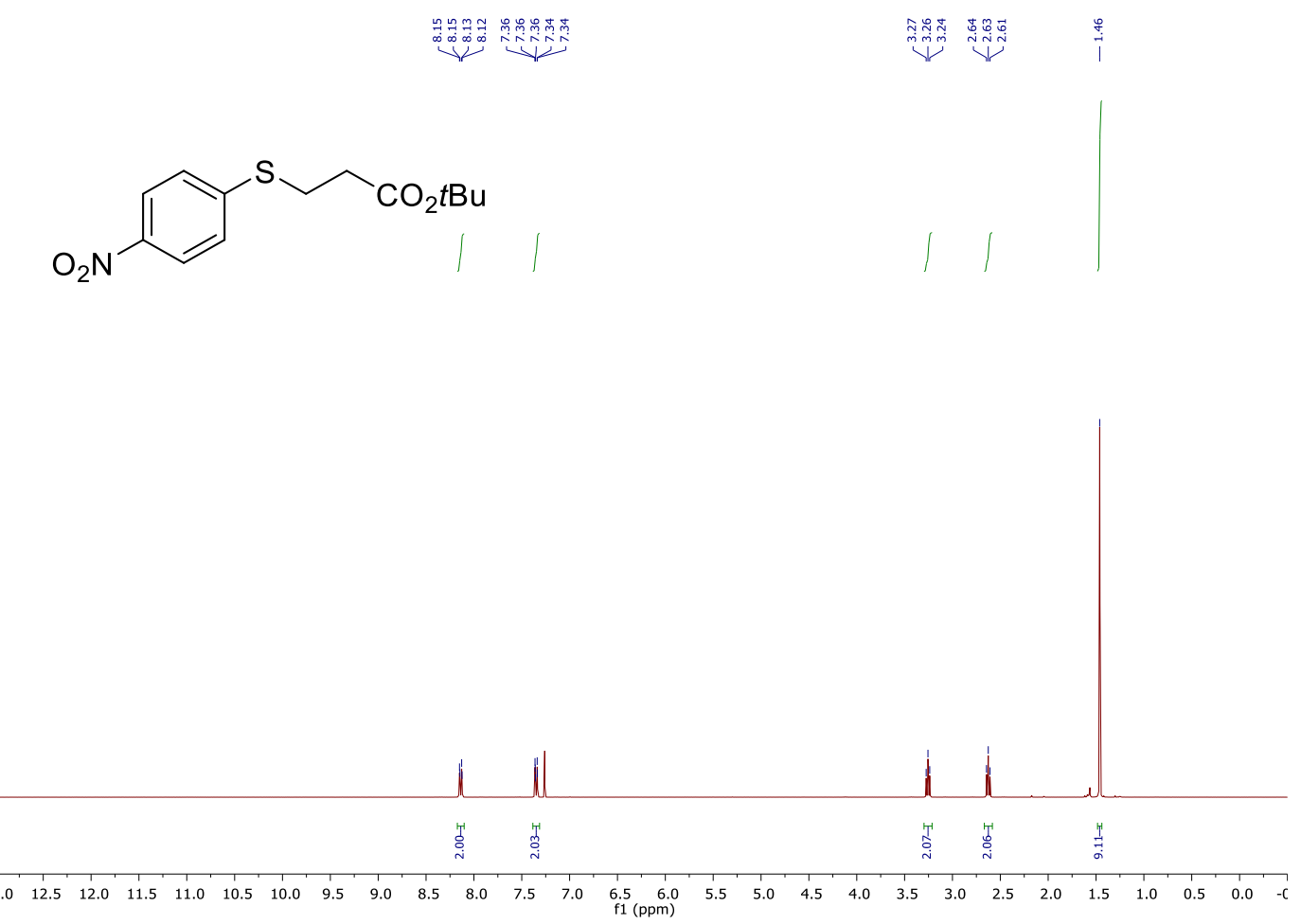

${ }^{13} \mathrm{C}$ NMR (101 MHz, Chloroform- $d$ )
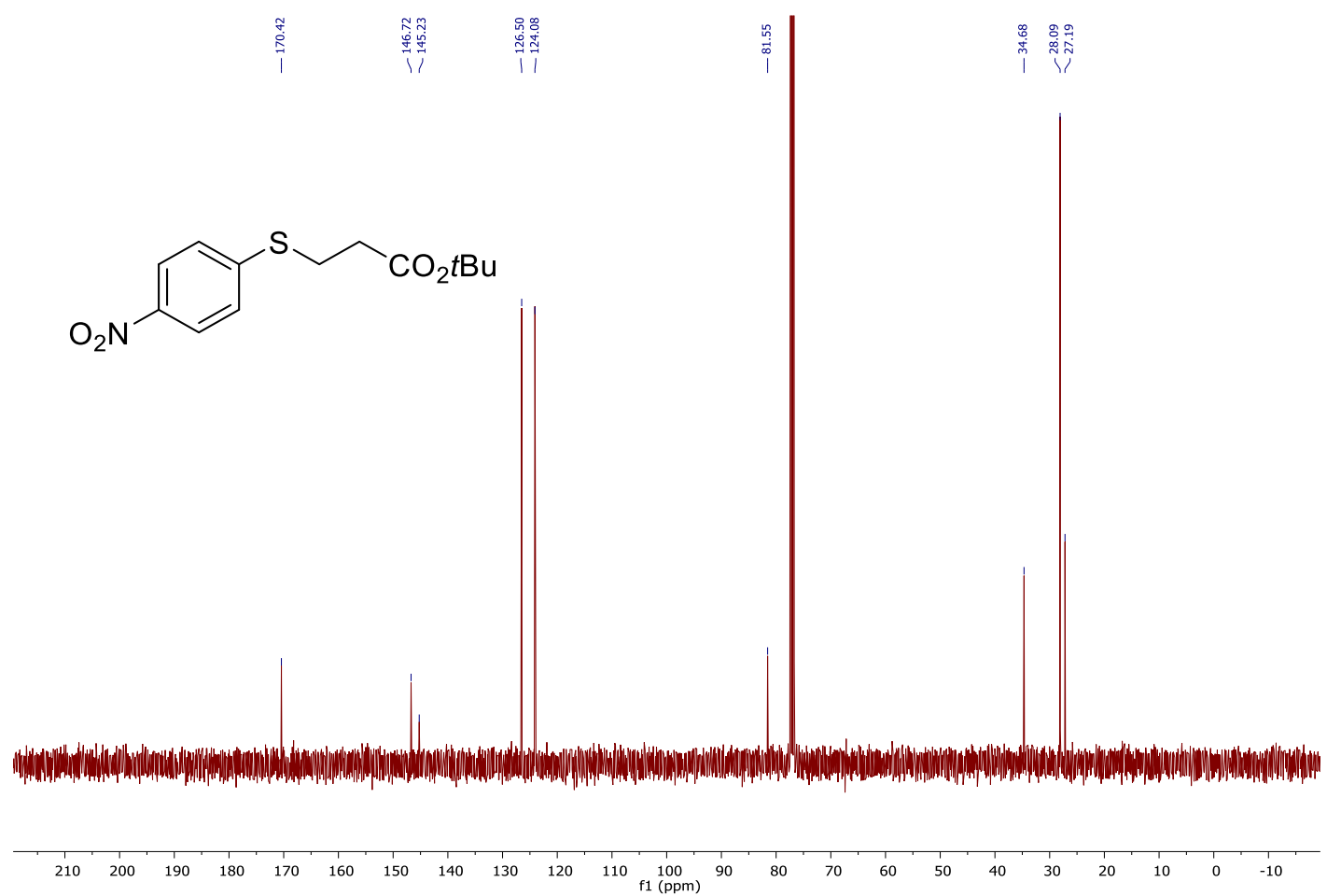


\section{Tert-butyl 3-(2-bromophenylthio)propanoate (21h)}

${ }^{1} \mathrm{H}$ NMR (400 MHz, Chloroform- $d$ )

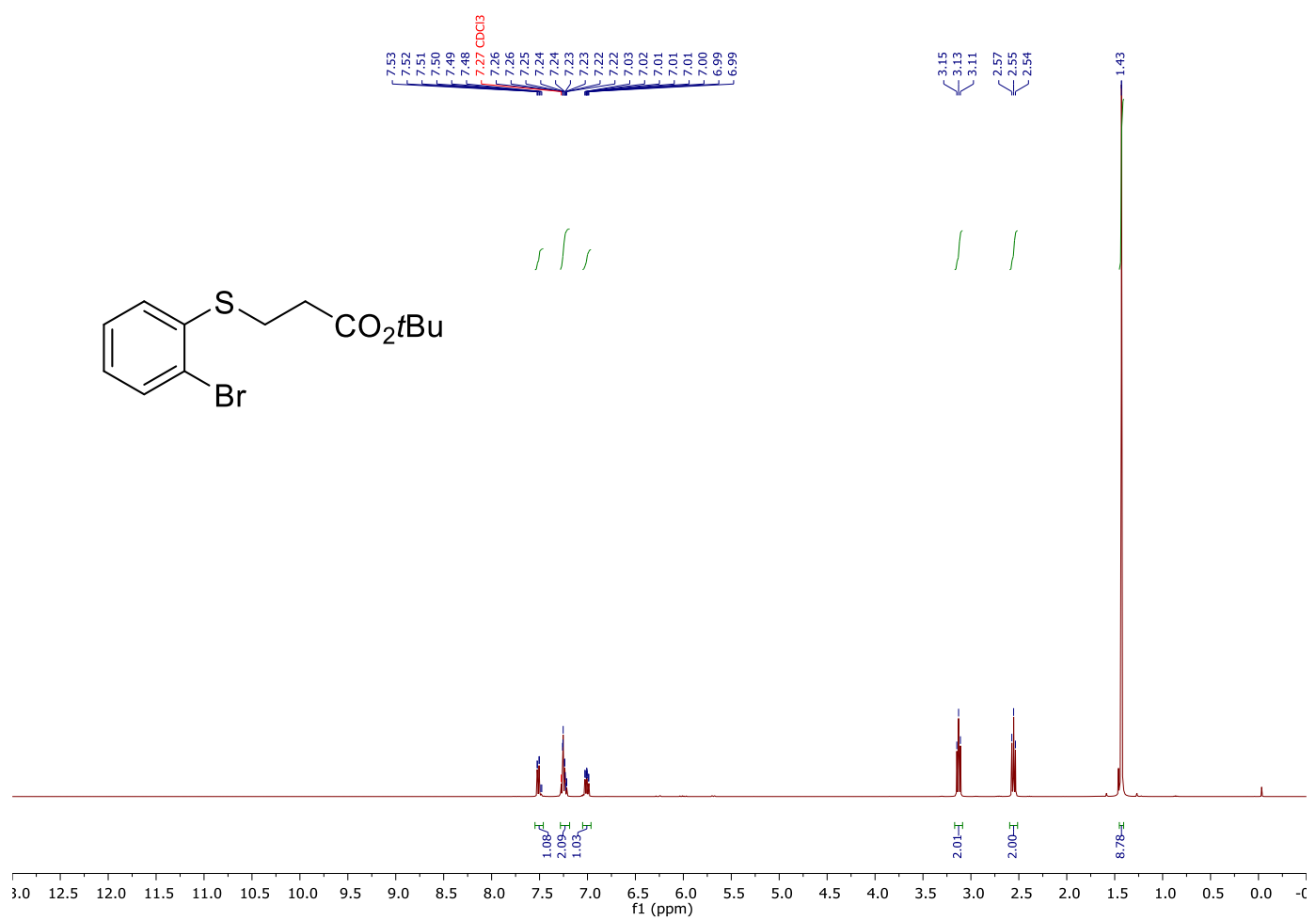

${ }^{13} \mathrm{C}$ NMR (101 MHz, Chloroform- $d$ )
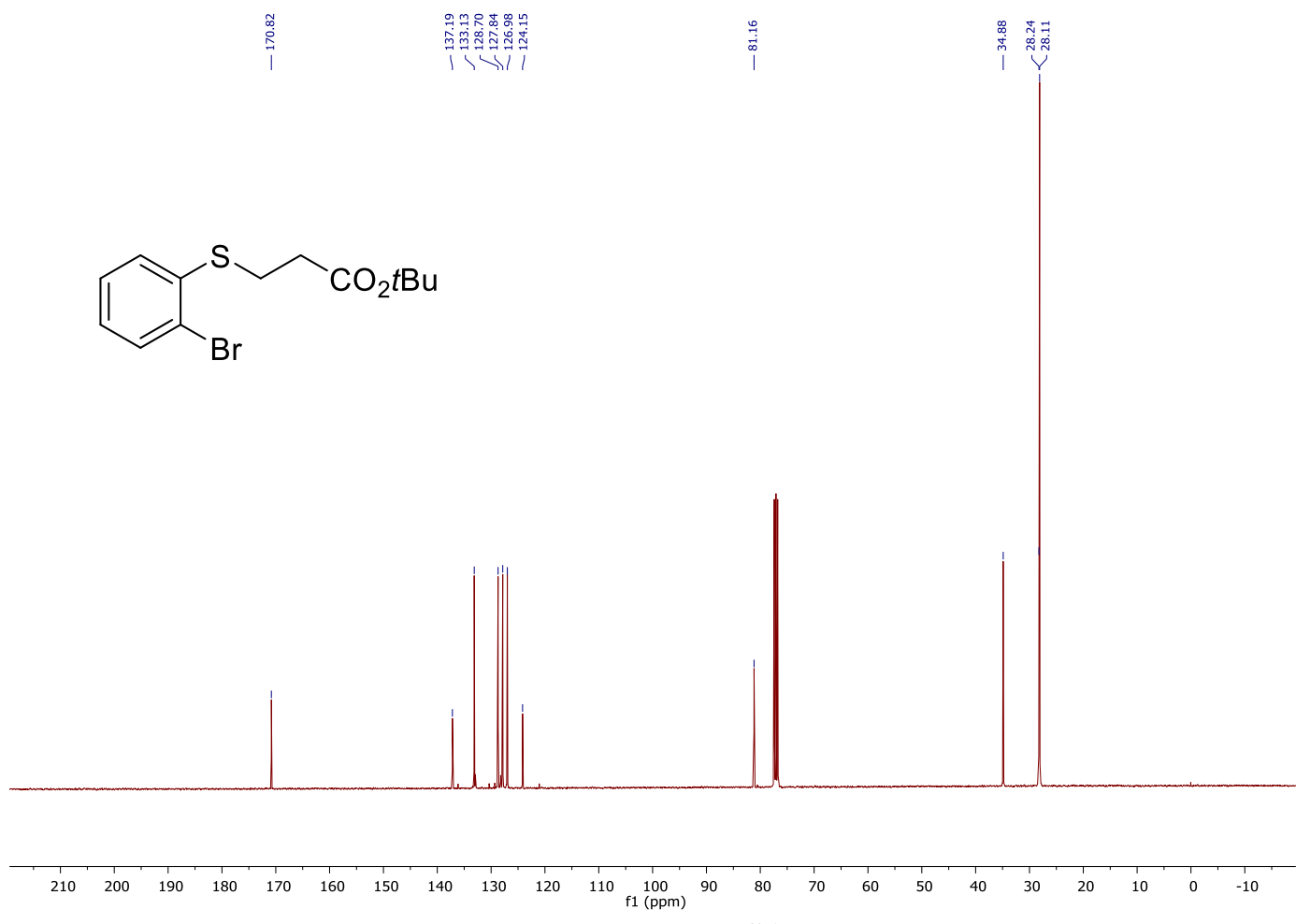


\section{Tert-butyl 3-(3-methylphenylthio)propanoate (21i)}

${ }^{1} \mathrm{H}$ NMR (400 MHz, Chloroform-d)

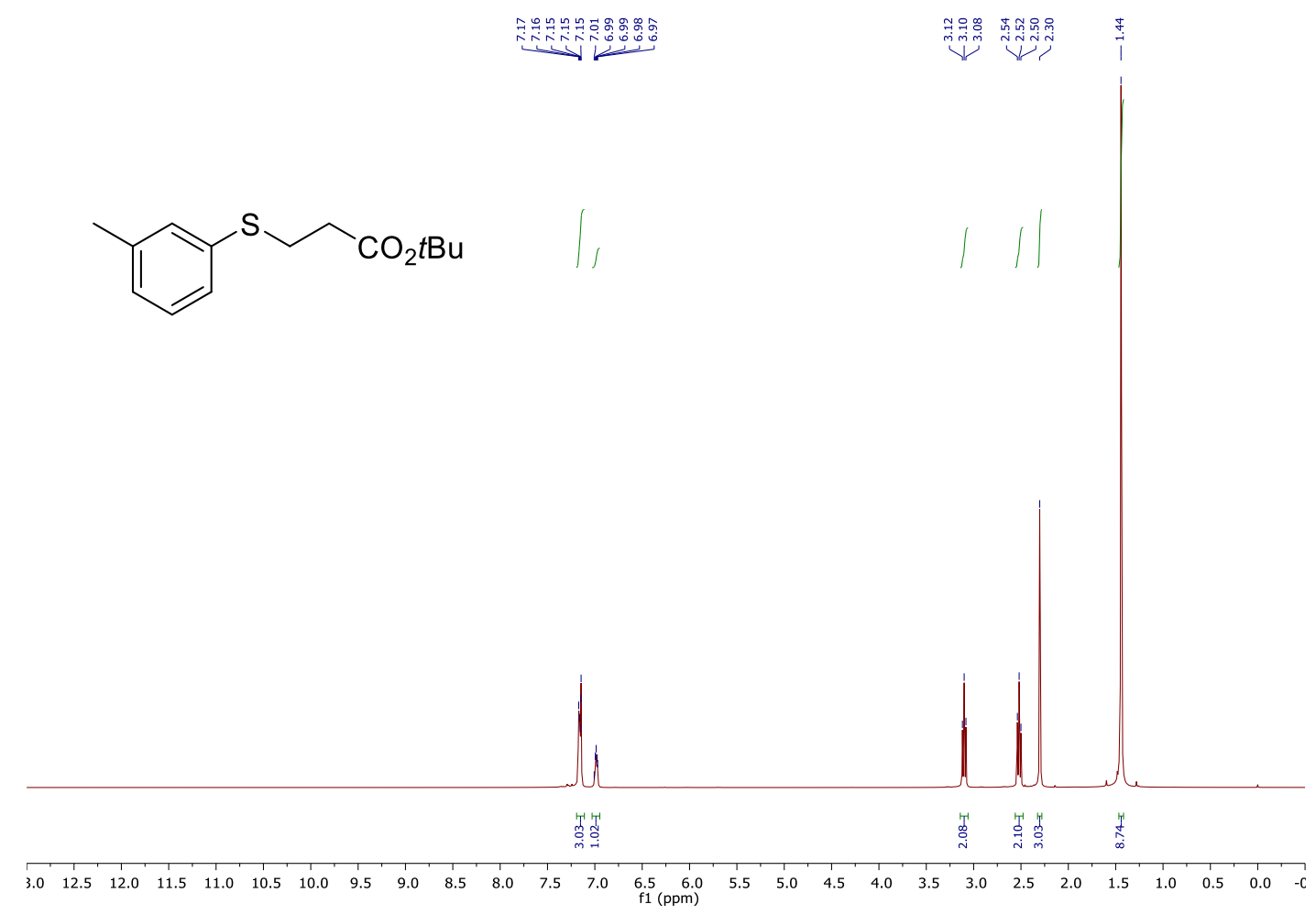

${ }^{13} \mathrm{C}$ NMR (101 MHz, Chloroform-d)
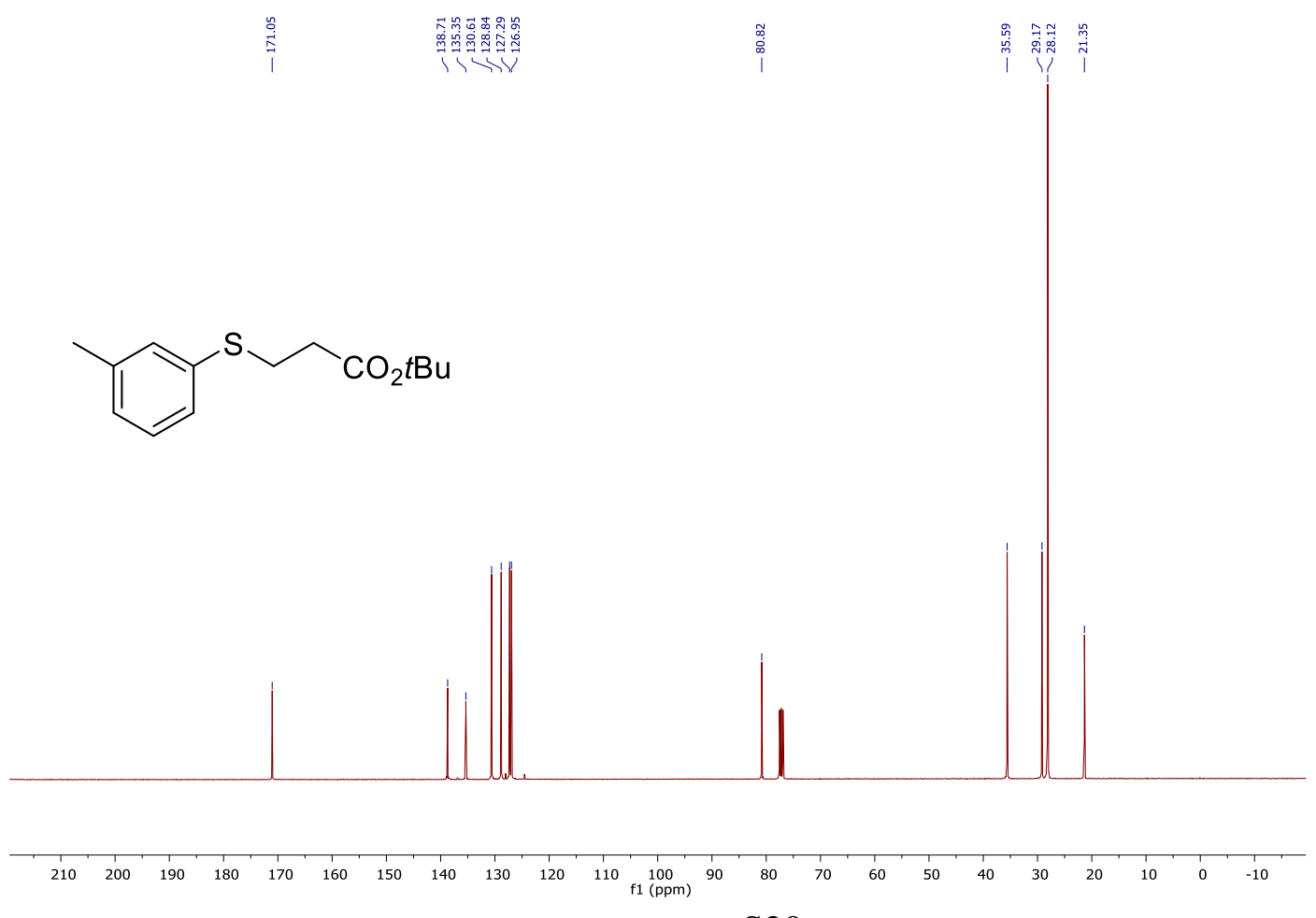
Tert-butyl 3-(3-bromophenylthio)propanoate (21j)

${ }^{1} \mathrm{H}$ NMR (400 MHz, Chloroform- $d$ )

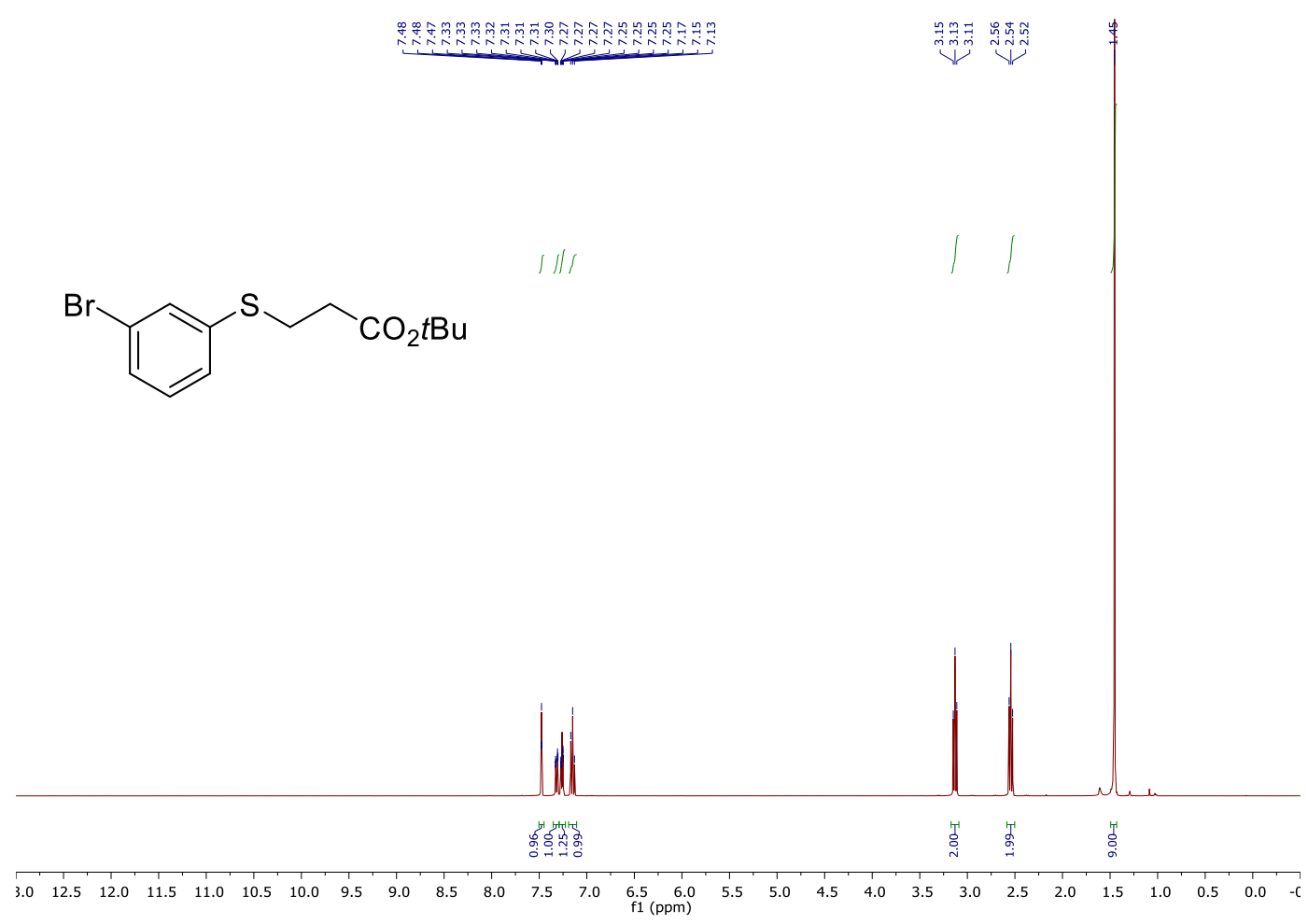

${ }^{13} \mathrm{C}$ NMR (101 MHz, Chloroform- $d$ )

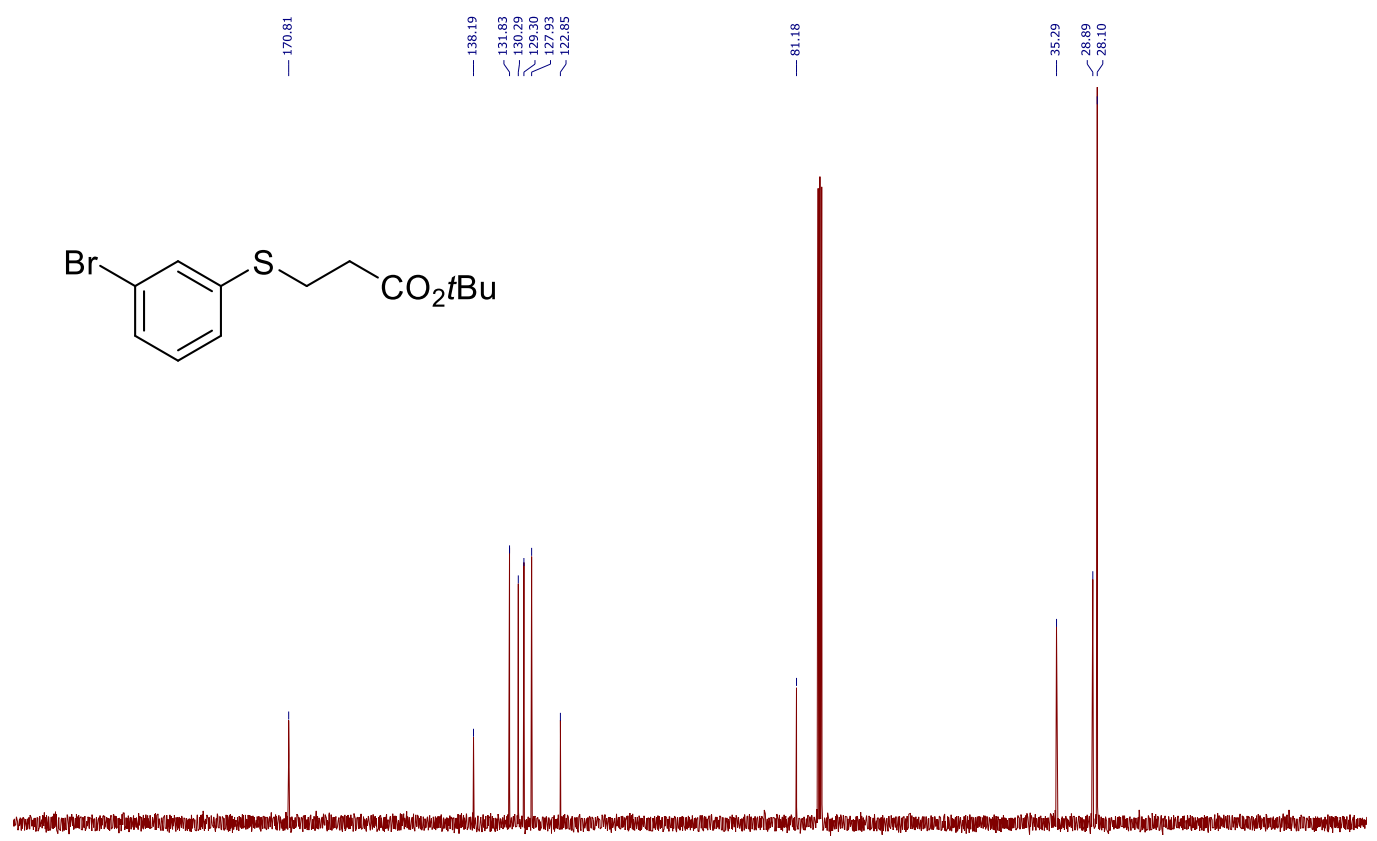

$\begin{array}{llllllllllllllllllllllll}210 & 200 & 190 & 180 & 170 & 160 & 150 & 140 & 130 & 120 & 110 & \underset{\mathrm{f} 1}{100}(\mathrm{ppm}) & 90 & 80 & 70 & 60 & 50 & 40 & 30 & 20 & 10 & 0 & -10\end{array}$ 


\section{Tert-butyl 3-((pyridin-2-yl)thio)propanoate (21k)}

${ }^{1} \mathrm{H}$ NMR (400 MHz, Chloroform- $d$ )

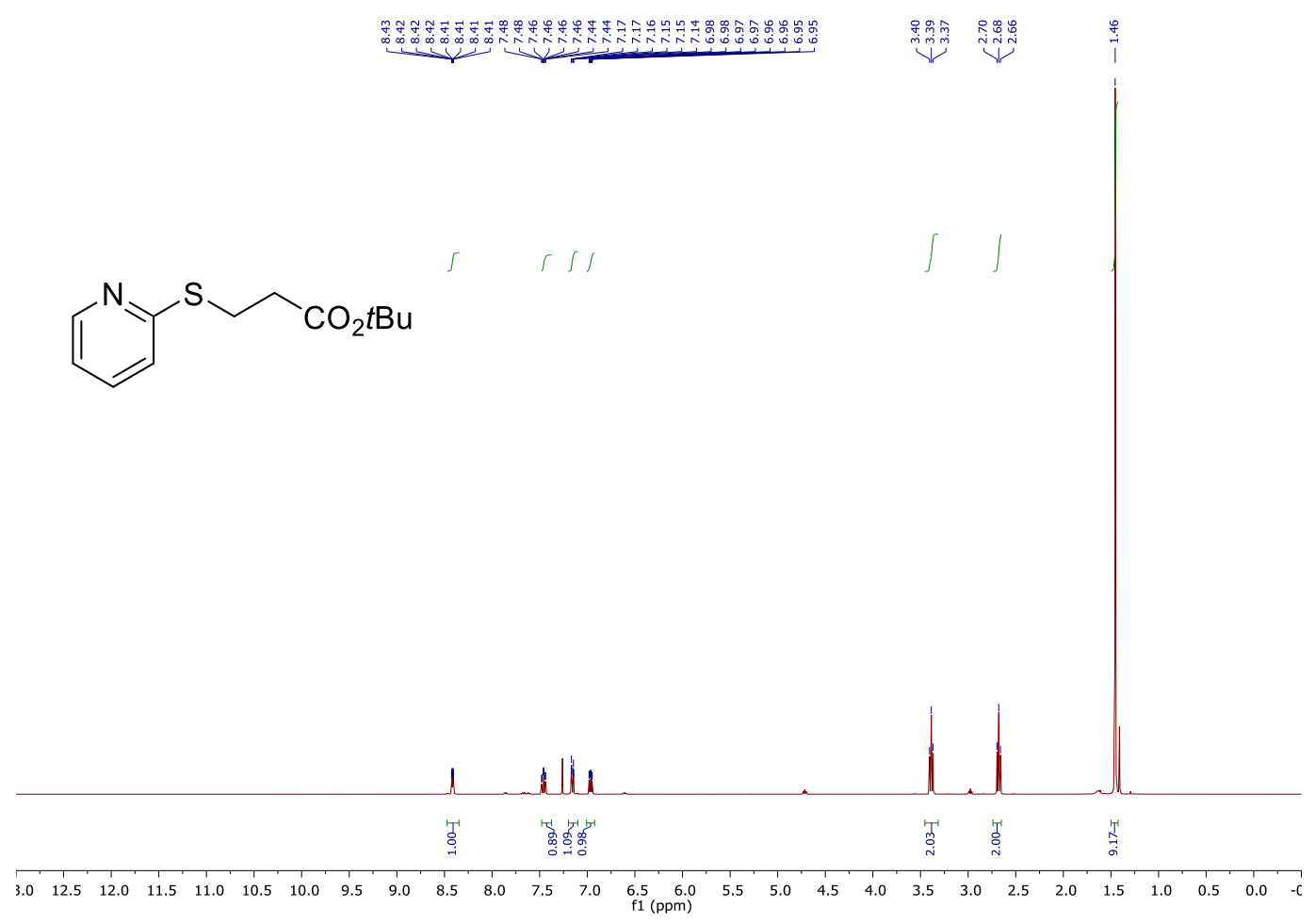

${ }^{13} \mathrm{C}$ NMR (101 MHz, Chloroform- $d$ )
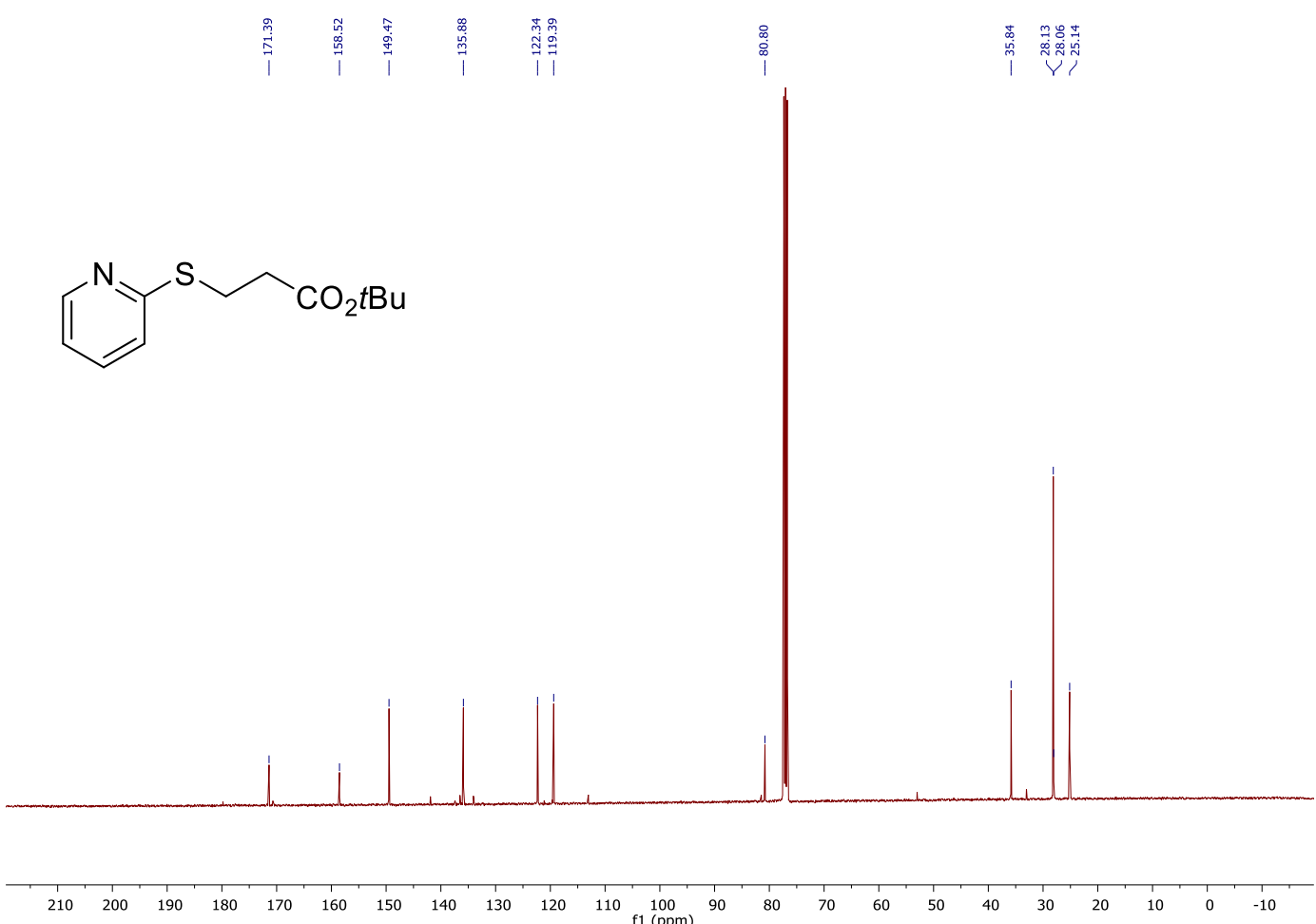


\section{Tert-butyl 3-((thiophen-2-yl)thio)propanoate (211)}

${ }^{1} \mathrm{H}$ NMR (400 MHz, Chloroform- $d$ )

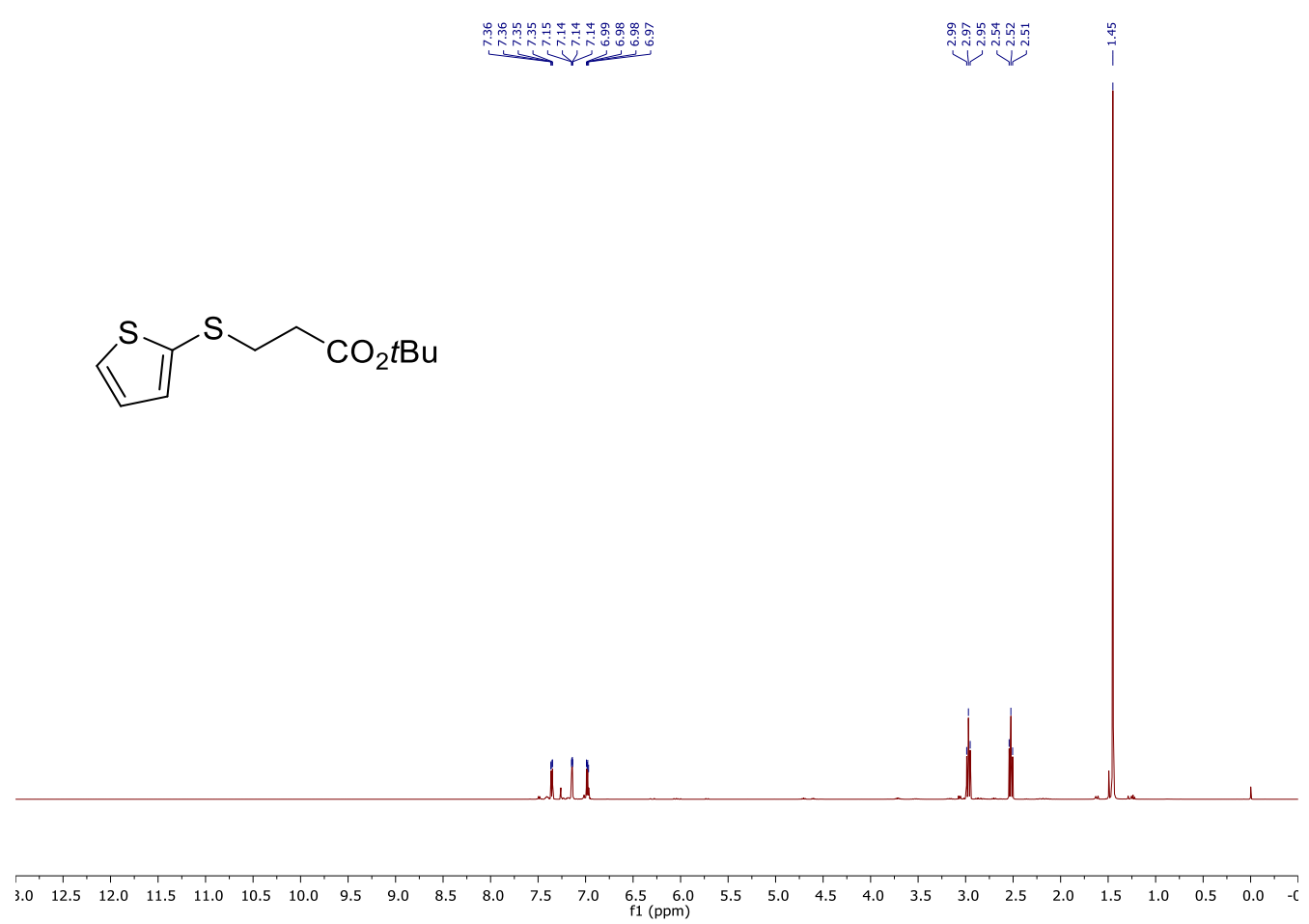

${ }^{13} \mathrm{C}$ NMR (101 MHz, Chloroform- $d$ )

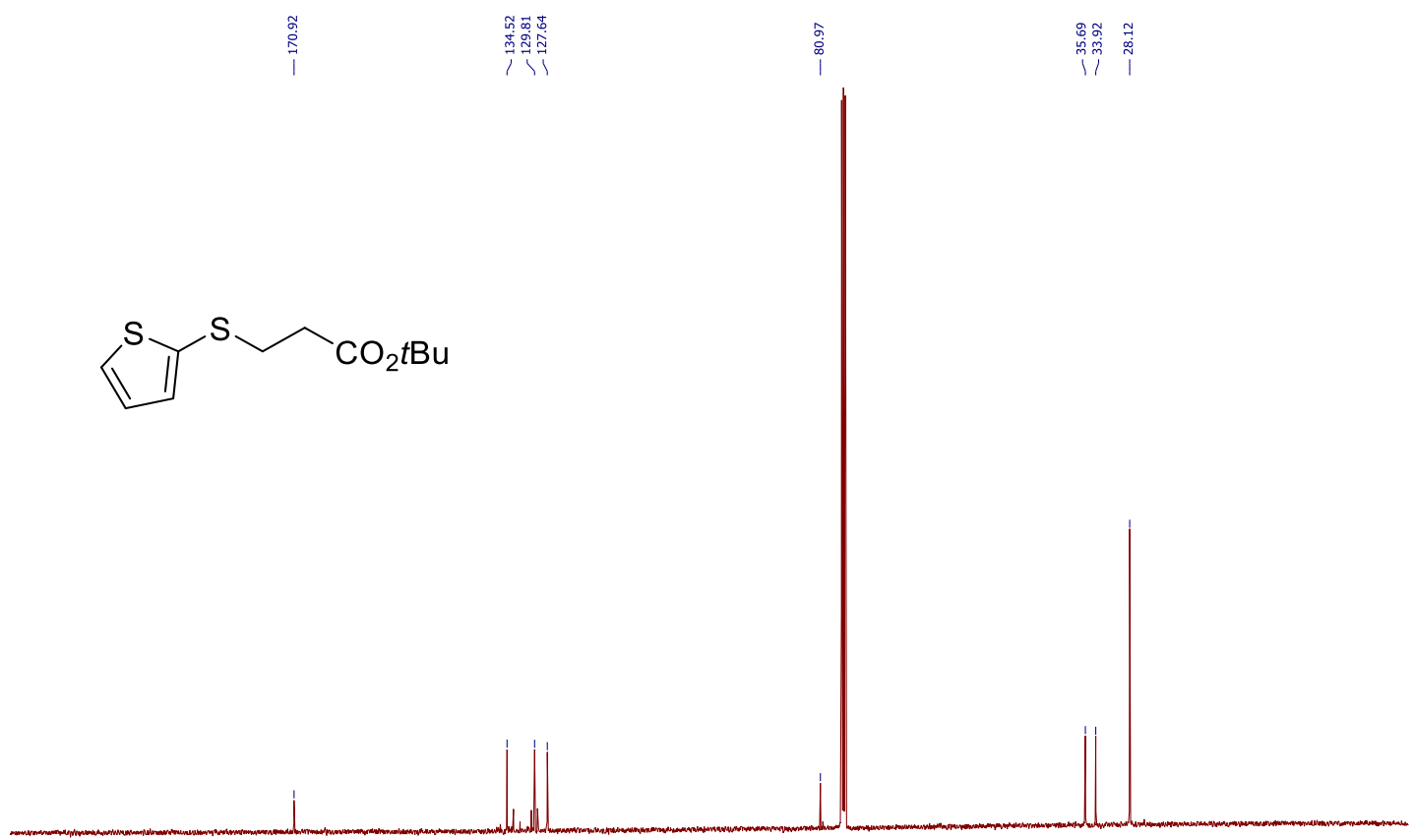

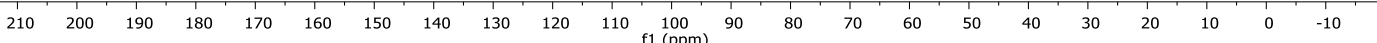




\section{Tert-butyl 3-((2-methylfuran-3-yl)thio)propanoate (21m)}

${ }^{1} \mathrm{H}$ NMR (400 MHz, Chloroform- $d$ )

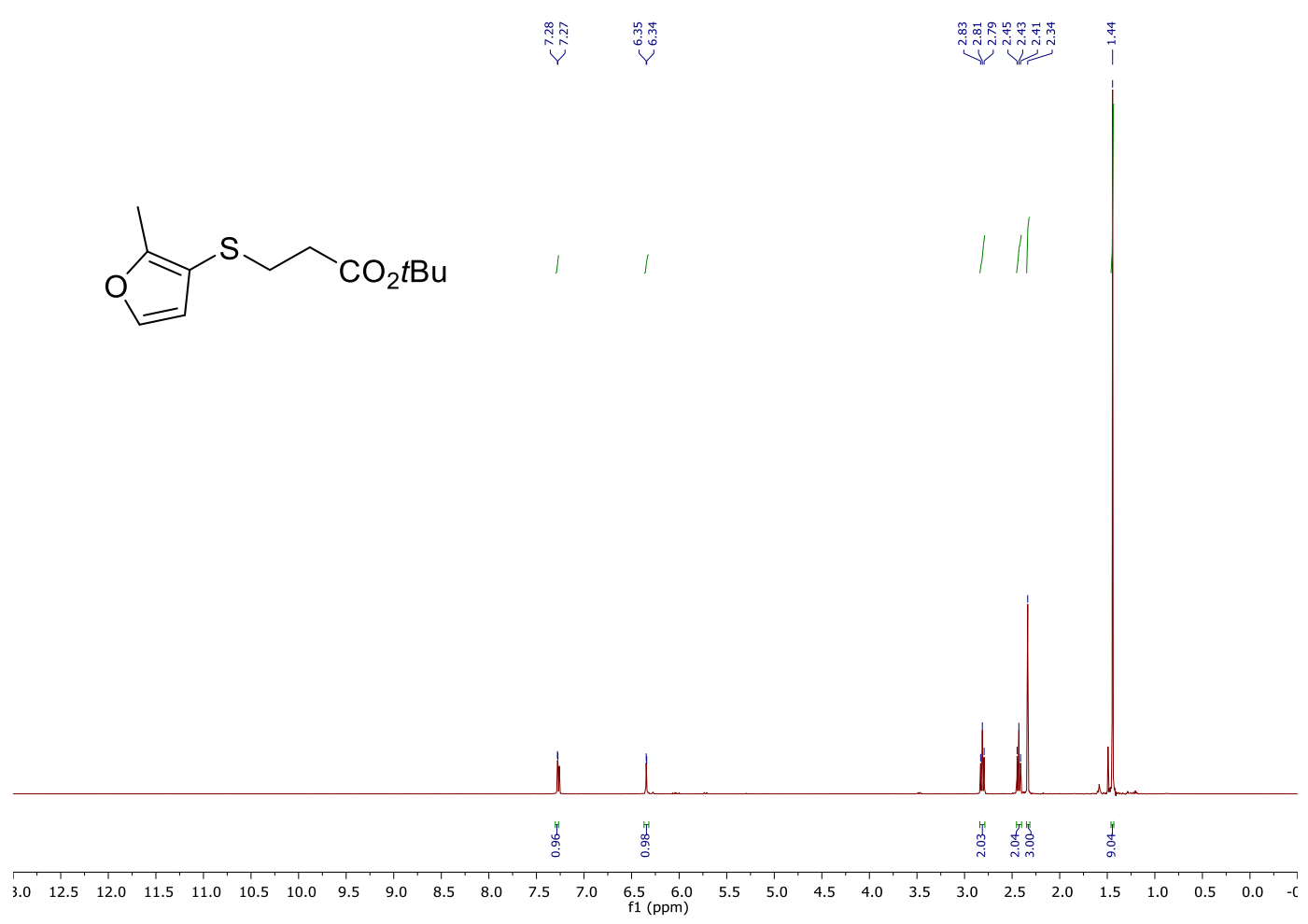

${ }^{13} \mathrm{C}$ NMR (101 MHz, Chloroform- $d$ )

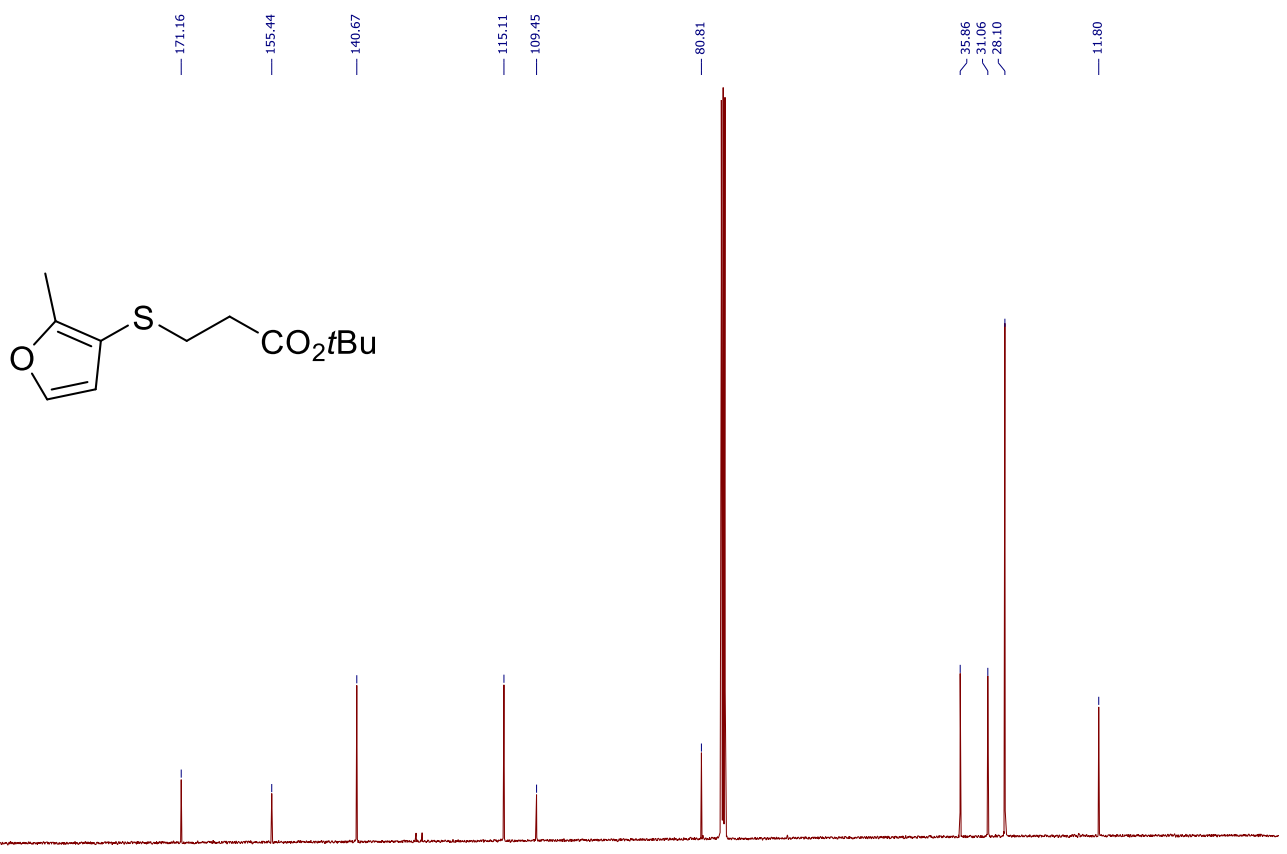

$\begin{array}{llllllllllllllllllllllll}\mid & 10 & 200 & 190 & 180 & 170 & 160 & 150 & 140 & 130 & 120 & 110 & 100 & 90 & 80 & 70 & 60 & 50 & 40 & 30 & 20 & 10 & 0 & -10\end{array}$ 


\section{Tert-butyl 3-(pentylthio)propanoate (21n)}

${ }^{1} \mathrm{H}$ NMR (400 MHz, Chloroform-d)

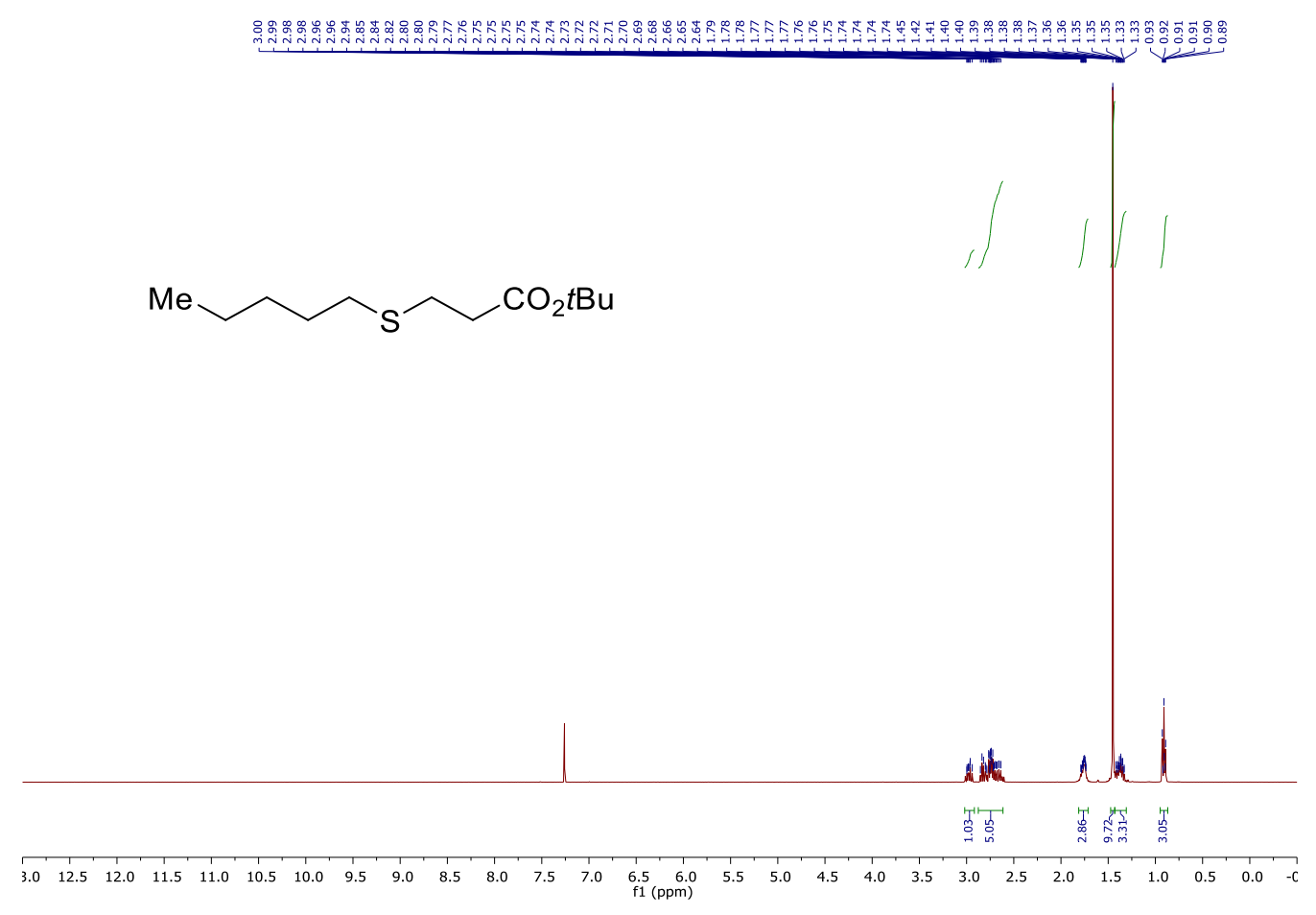

${ }^{13} \mathrm{C}$ NMR (101 MHz, Chloroform-d)

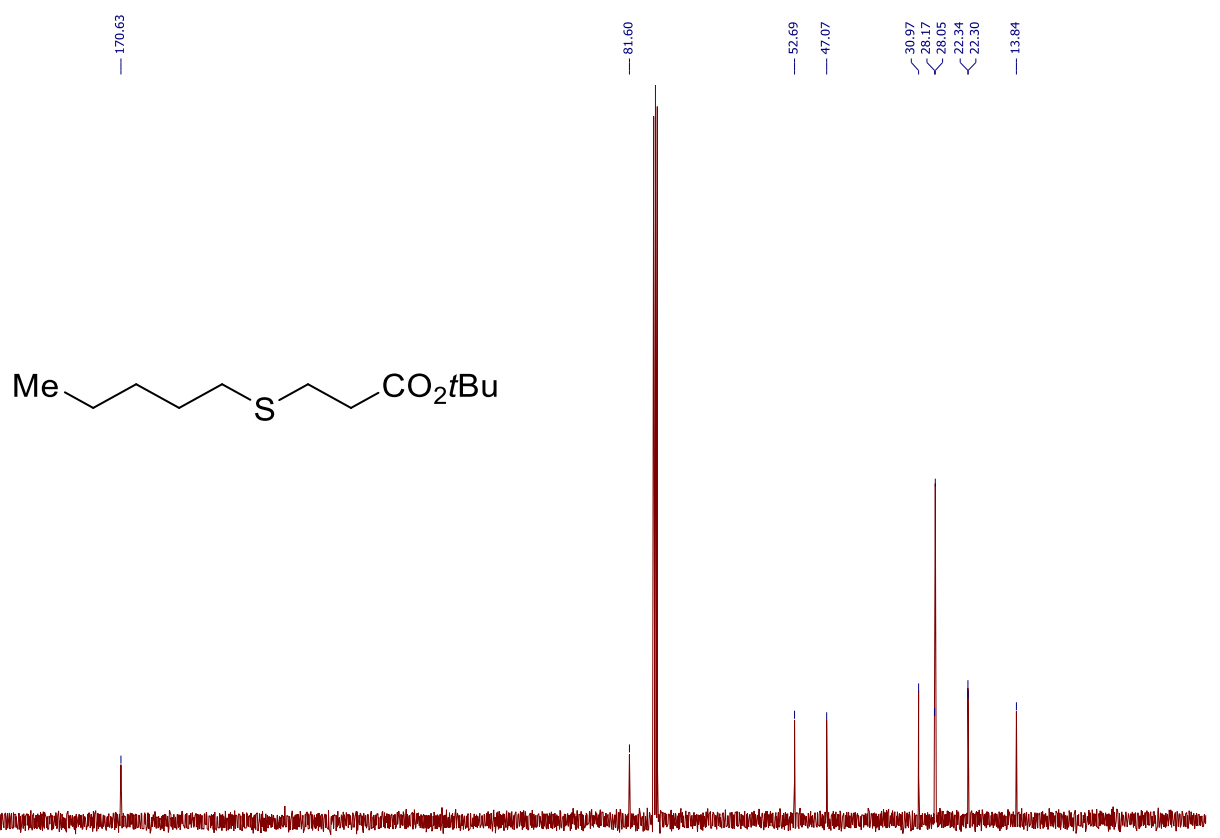

$\begin{array}{lllllllllllllllllllllll}210 & 200 & 190 & 180 & 170 & 160 & 150 & 140 & 130 & 120 & 110 & \begin{array}{c}100 \\ \mathrm{f} 1(\mathrm{ppm})\end{array} & 90 & 80 & 70 & 60 & 50 & 40 & 30 & 20 & 10 & 0 & -10\end{array}$ 


\section{Tert-butyl 3-(cyclohexylthio)propanoate (210)}

${ }^{1} \mathrm{H}$ NMR (400 MHz, Chloroform- $d$ )

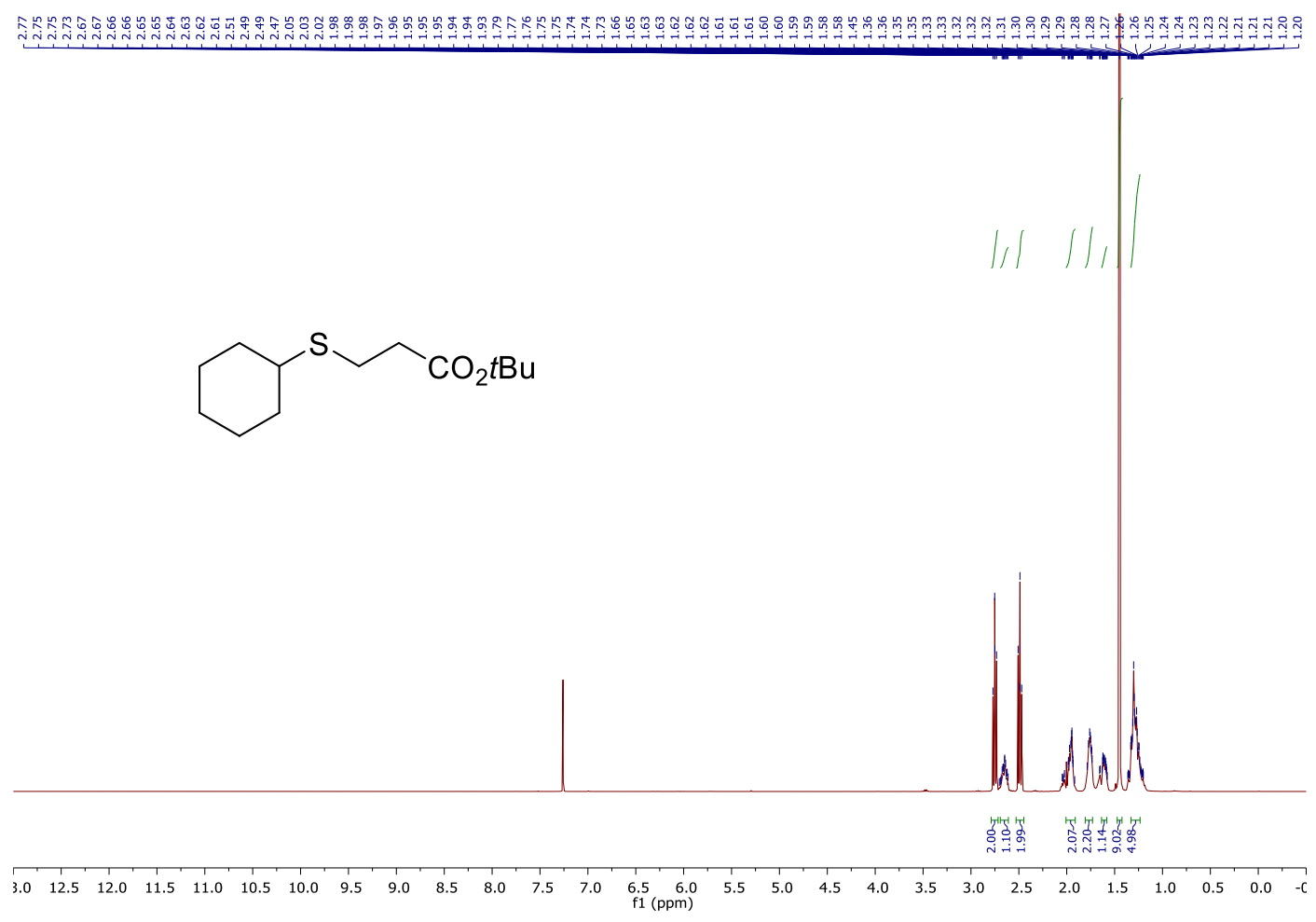

${ }^{13} \mathrm{C}$ NMR (101 MHz, Chloroform- $d$ )

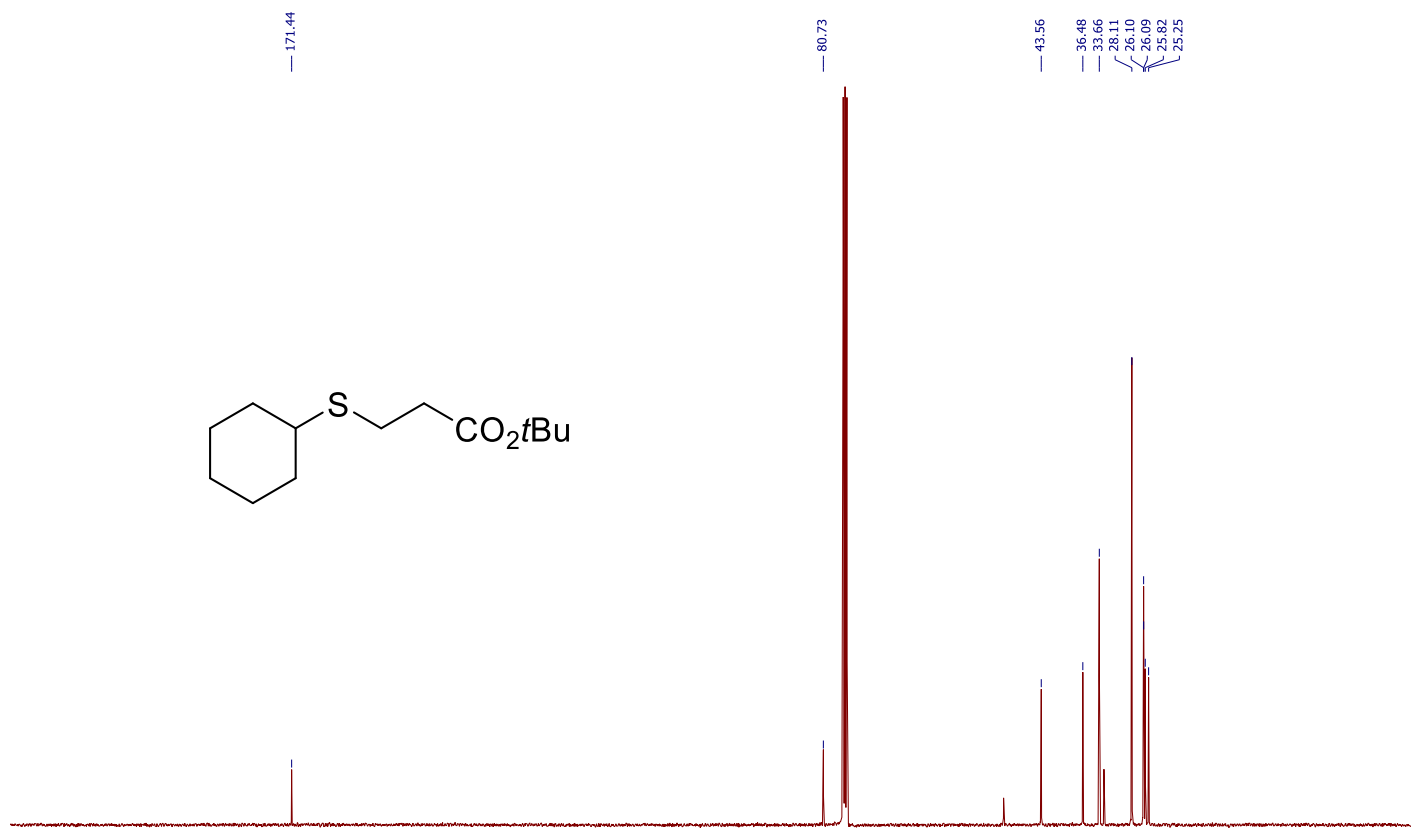

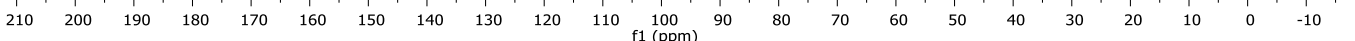




\section{Tert-butyl 3-((4-methylphenyl)sulfinyl)propanoate (6a)}

${ }^{1} \mathrm{H}$ NMR (400 MHz, Chloroform- $d$ )

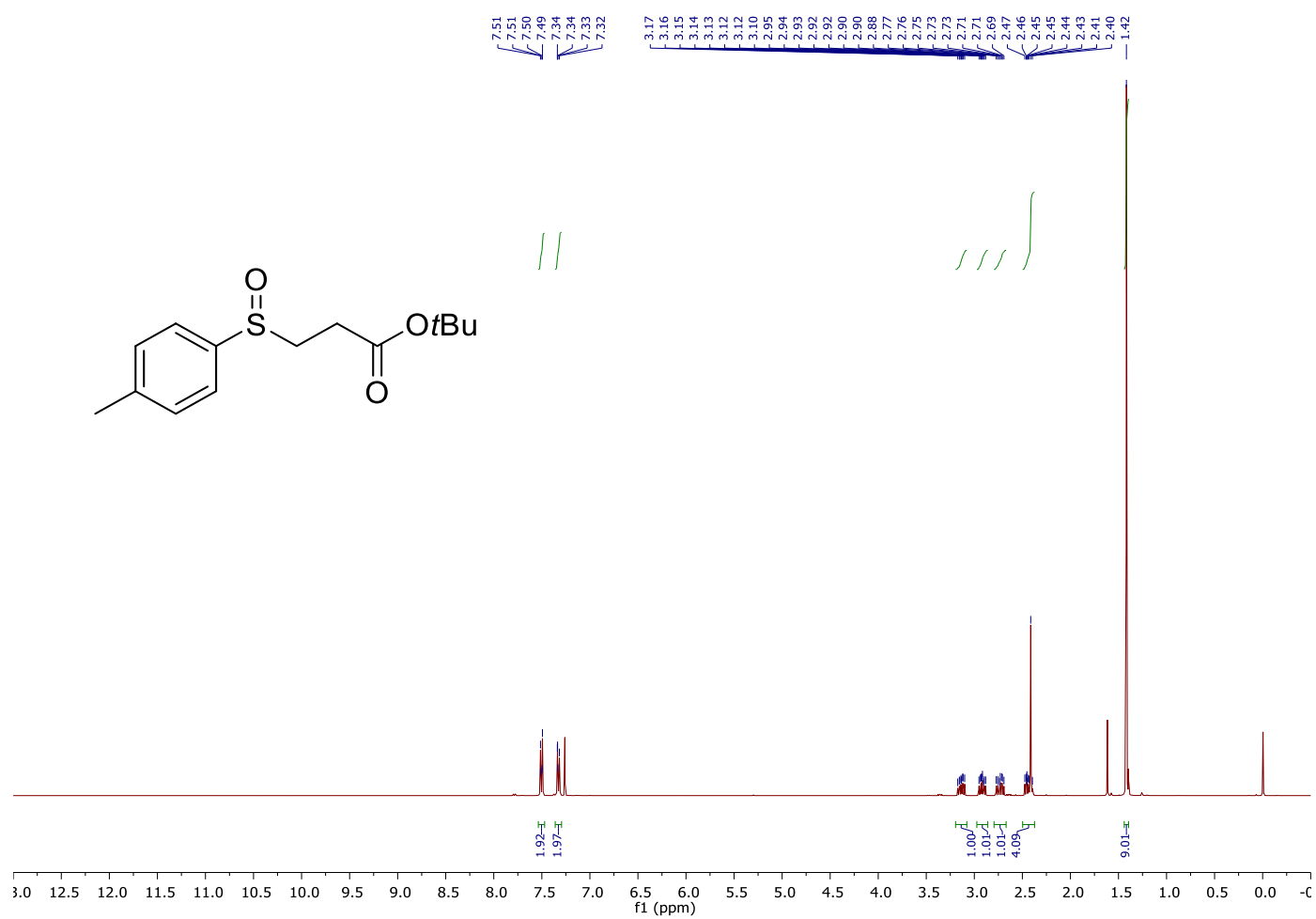

${ }^{13} \mathrm{C}$ NMR (101 MHz, Chloroform- $d$ )

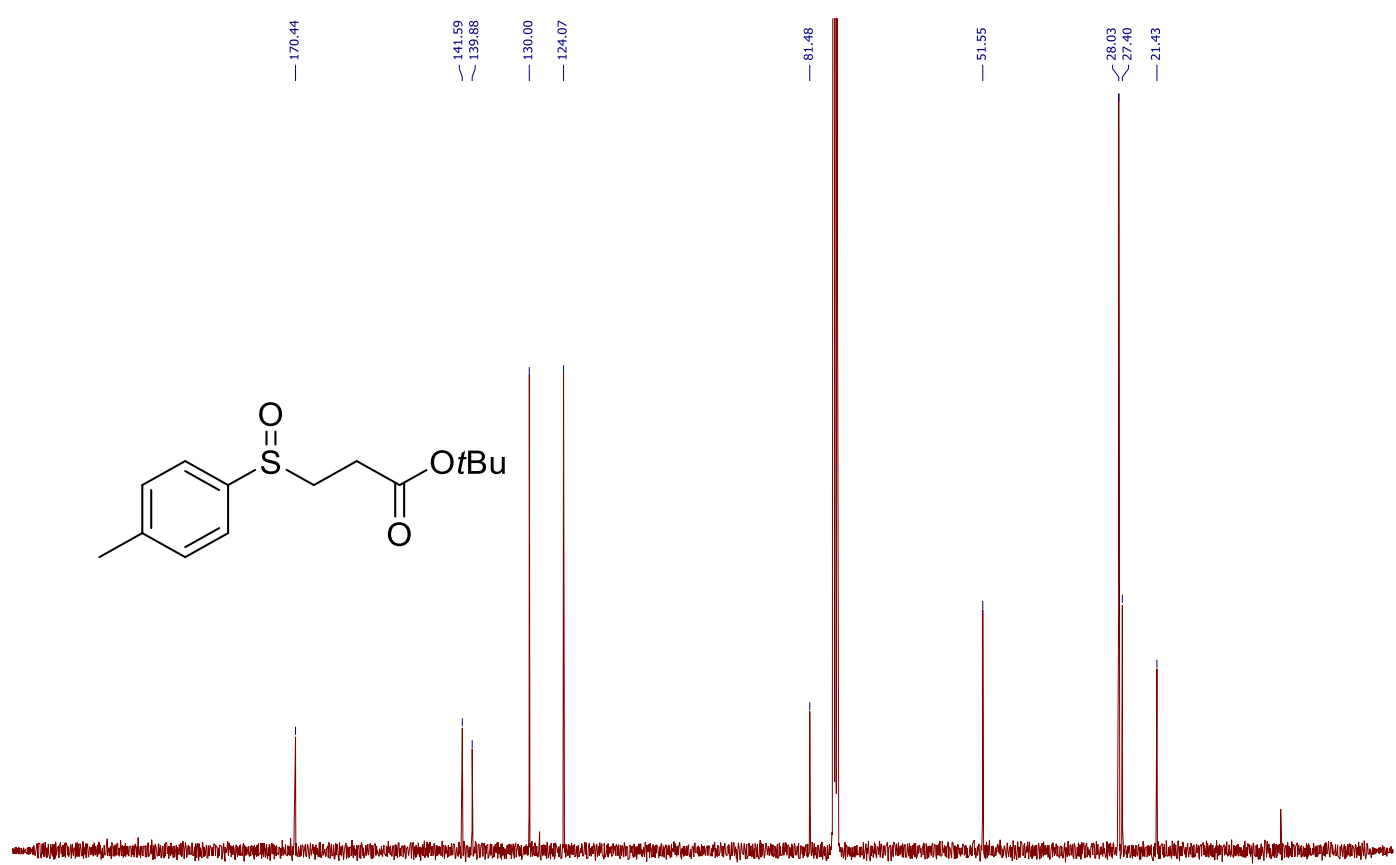

$\begin{array}{llllllllllllllllllllllll}210 & 200 & 190 & 180 & 170 & 160 & 150 & 140 & 130 & 120 & 110 & 100 & 90 & 80 & 70 & 60 & 50 & 40 & 30 & 20 & 10 & 0 & -10\end{array}$ 


\section{Tert-butyl 3-[(phenyl)sulfinyl]propanoate (6b)}

${ }^{1} \mathrm{H}$ NMR (400 MHz, Chloroform- $d$ )

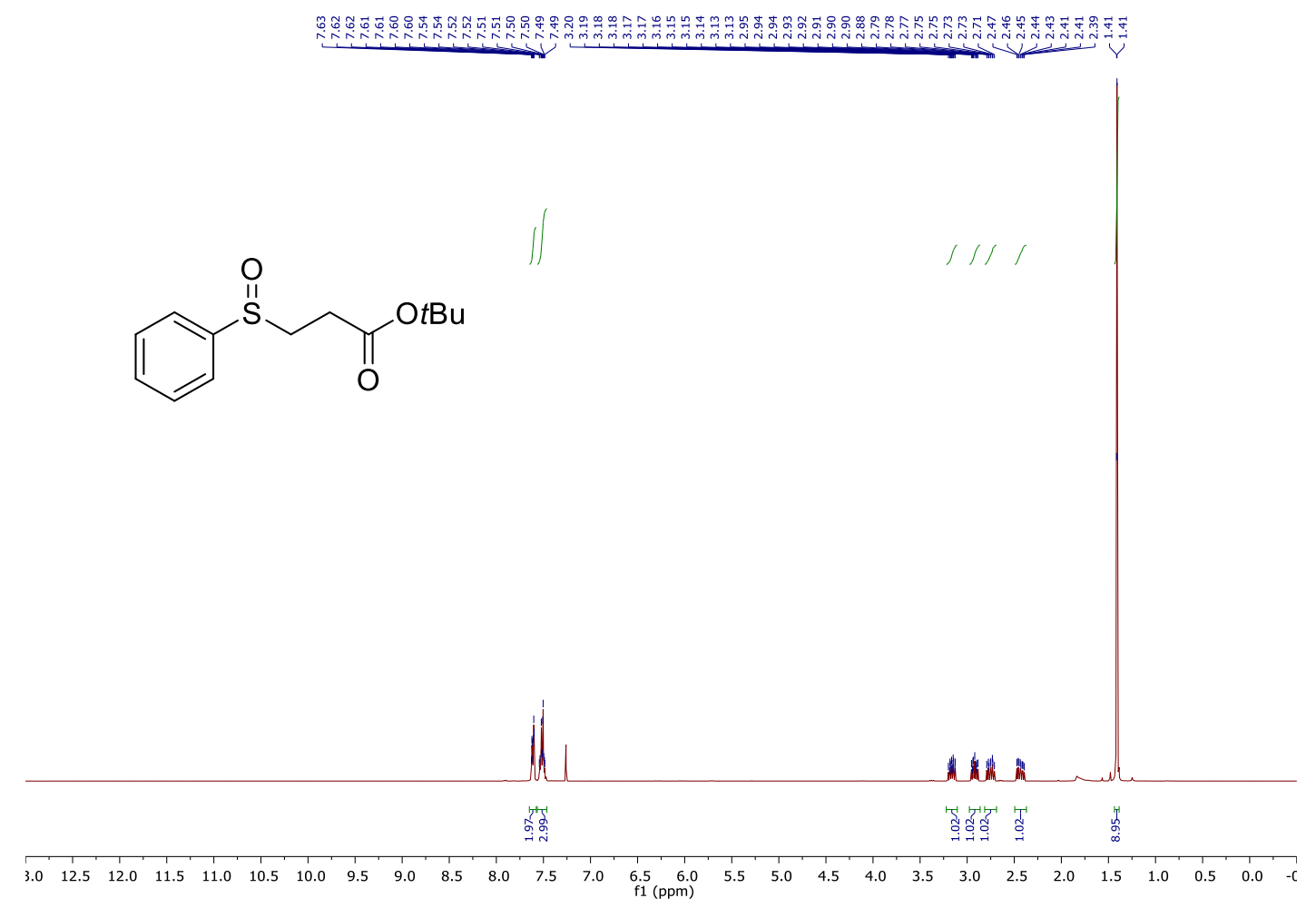

${ }^{13} \mathrm{C}$ NMR (101 MHz, Chloroform- $d$ )
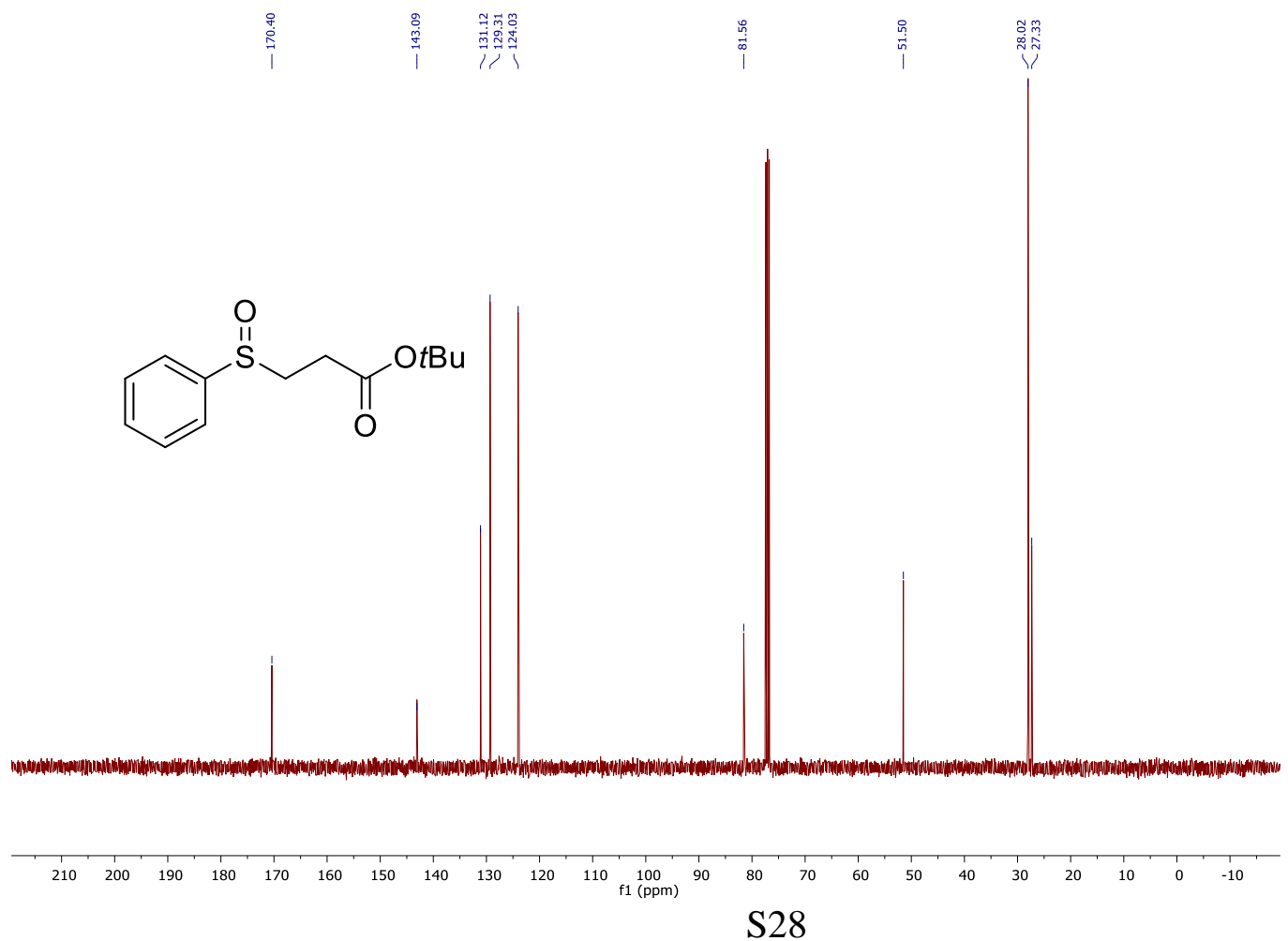


\section{Tert-butyl 3-[(4-methoxyphenyl)sulfinyl]propanoate (6c)}

${ }^{1} \mathrm{H}$ NMR (400 MHz, Chloroform- $d$ )

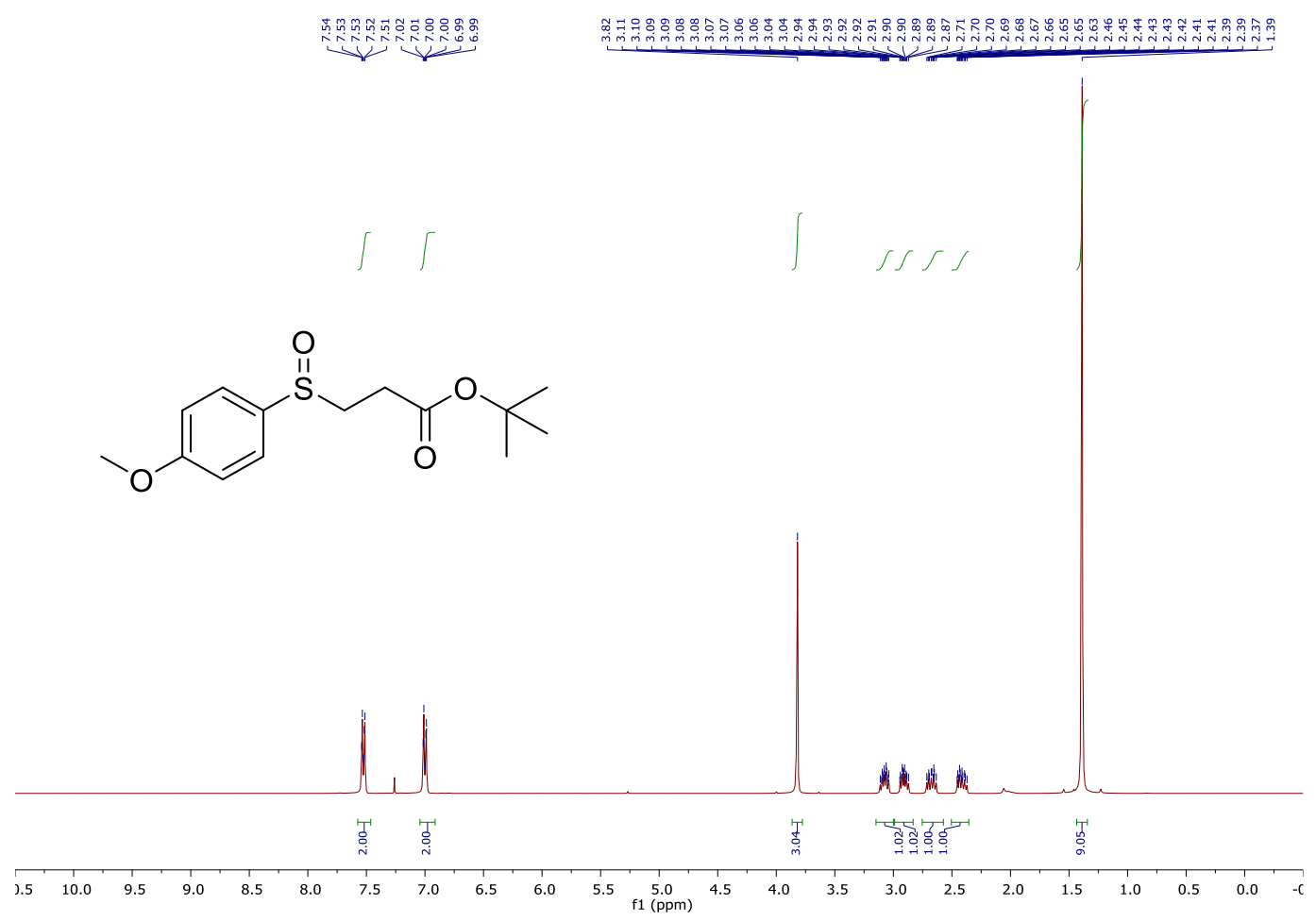

${ }^{13} \mathrm{C}$ NMR (101 MHz, Chloroform- $d$ )
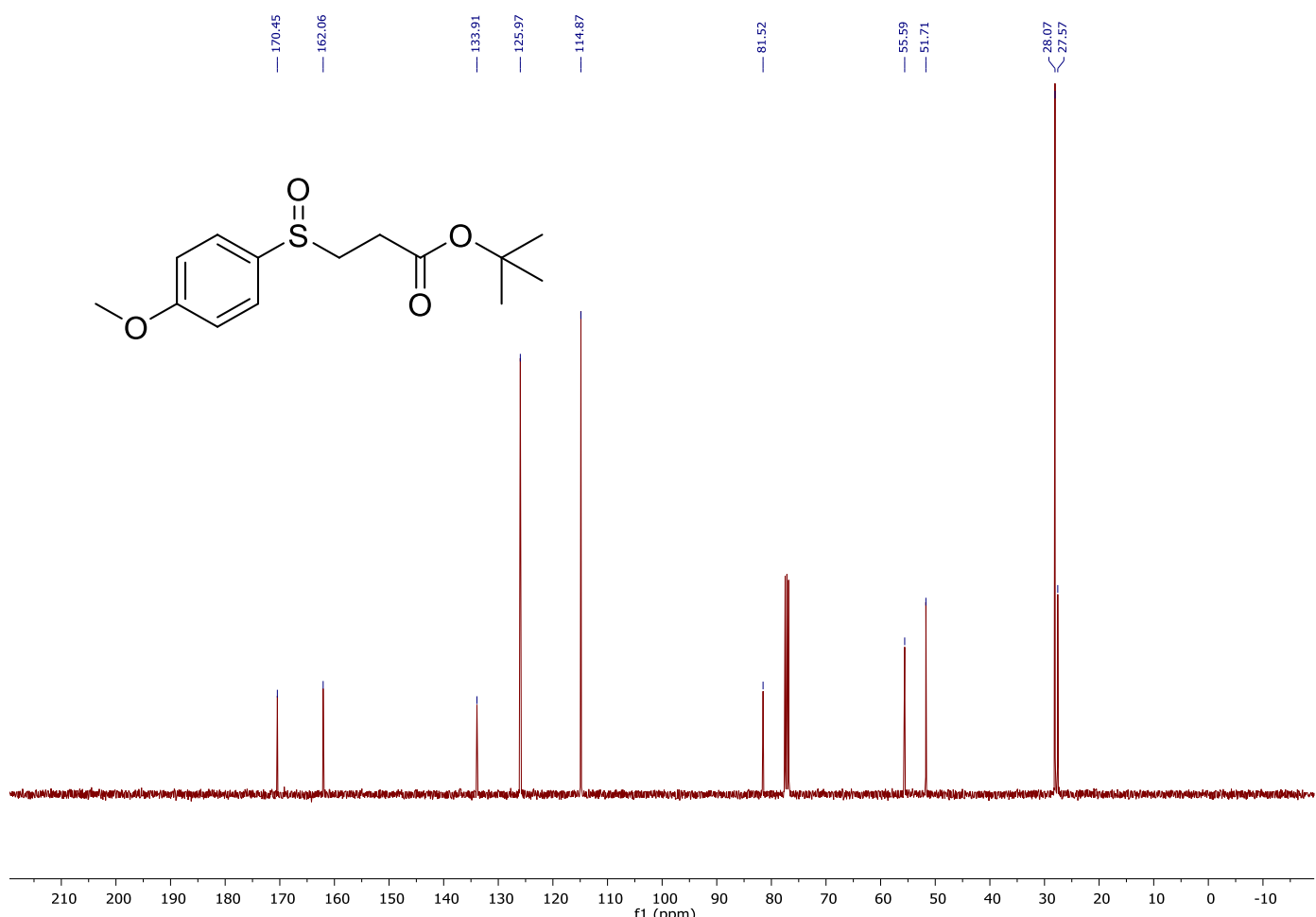


\section{Tert-butyl 3-[(4-bromophenyl)sulfinyl]propanoate (6d)}

${ }^{1} \mathrm{H}$ NMR (400 MHz, Chloroform- $d$ )

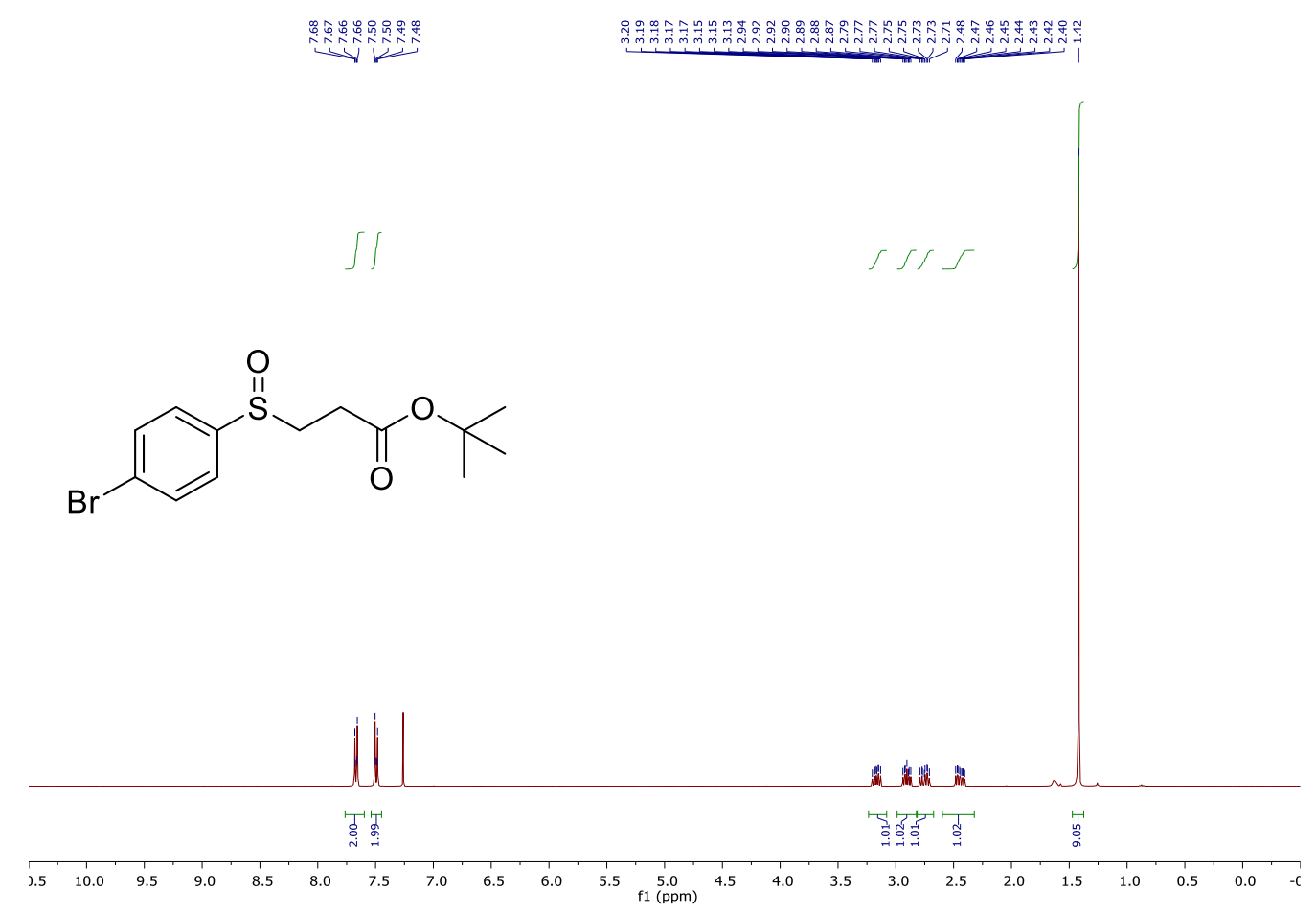

${ }^{13} \mathrm{C}$ NMR (101 MHz, Chloroform- $d$ )
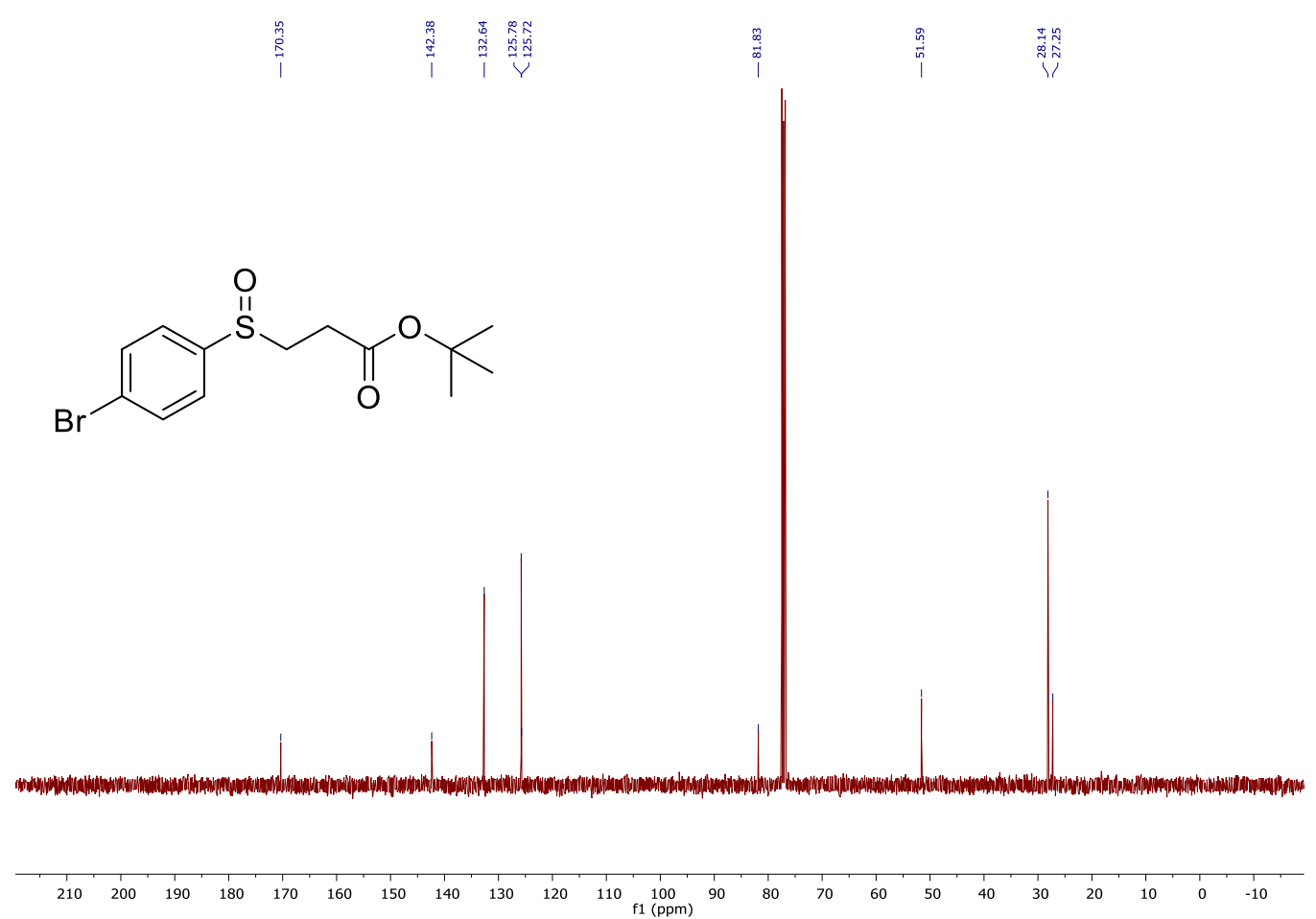


\section{Tert-butyl 3-[(4-fluorophenyl)sulfinyl]propanoate (6e)}

${ }^{1} \mathrm{H}$ NMR (400 MHz, Chloroform- $d$ )

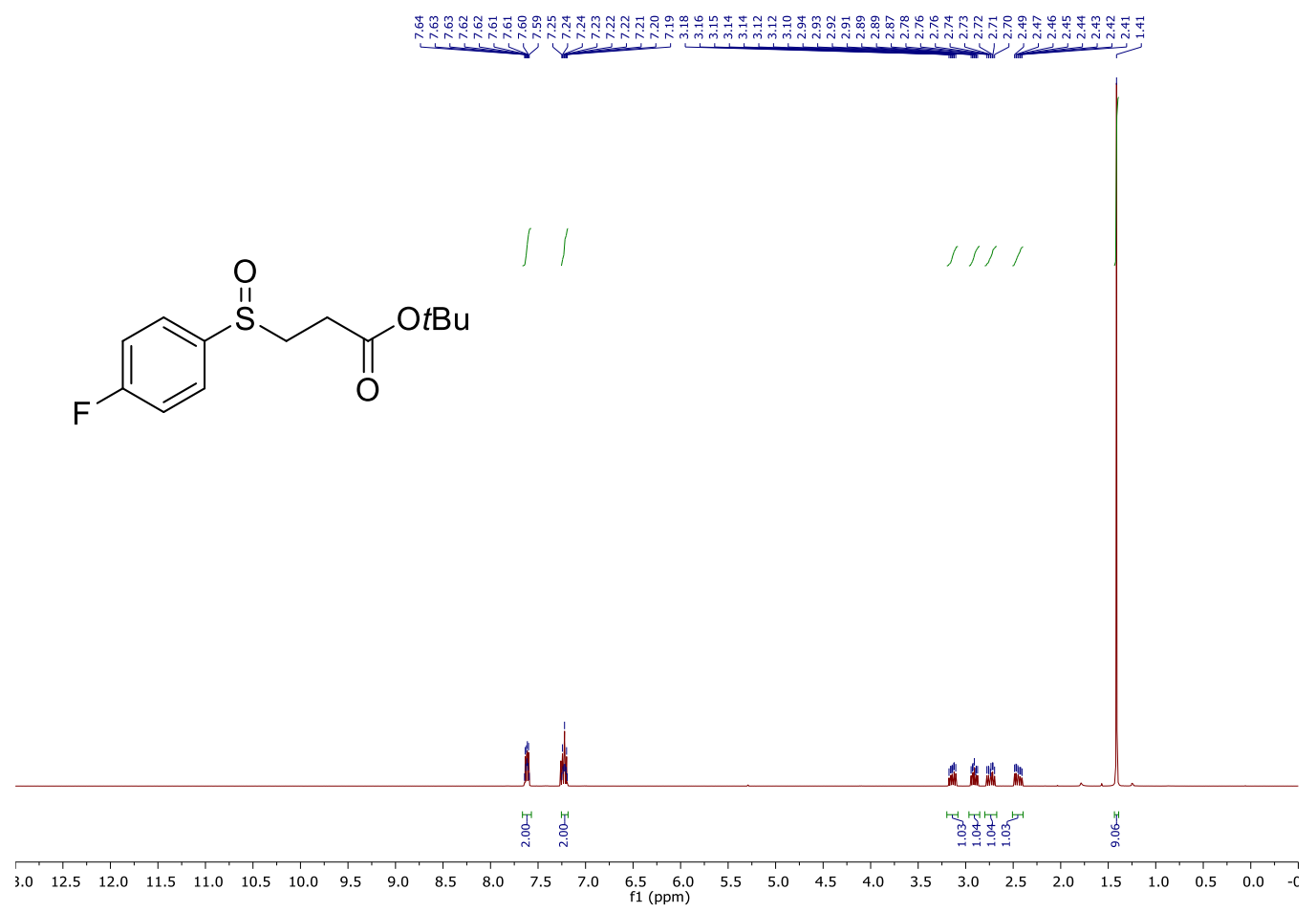

${ }^{13} \mathrm{C}$ NMR (101 MHz, Chloroform- $d$ )

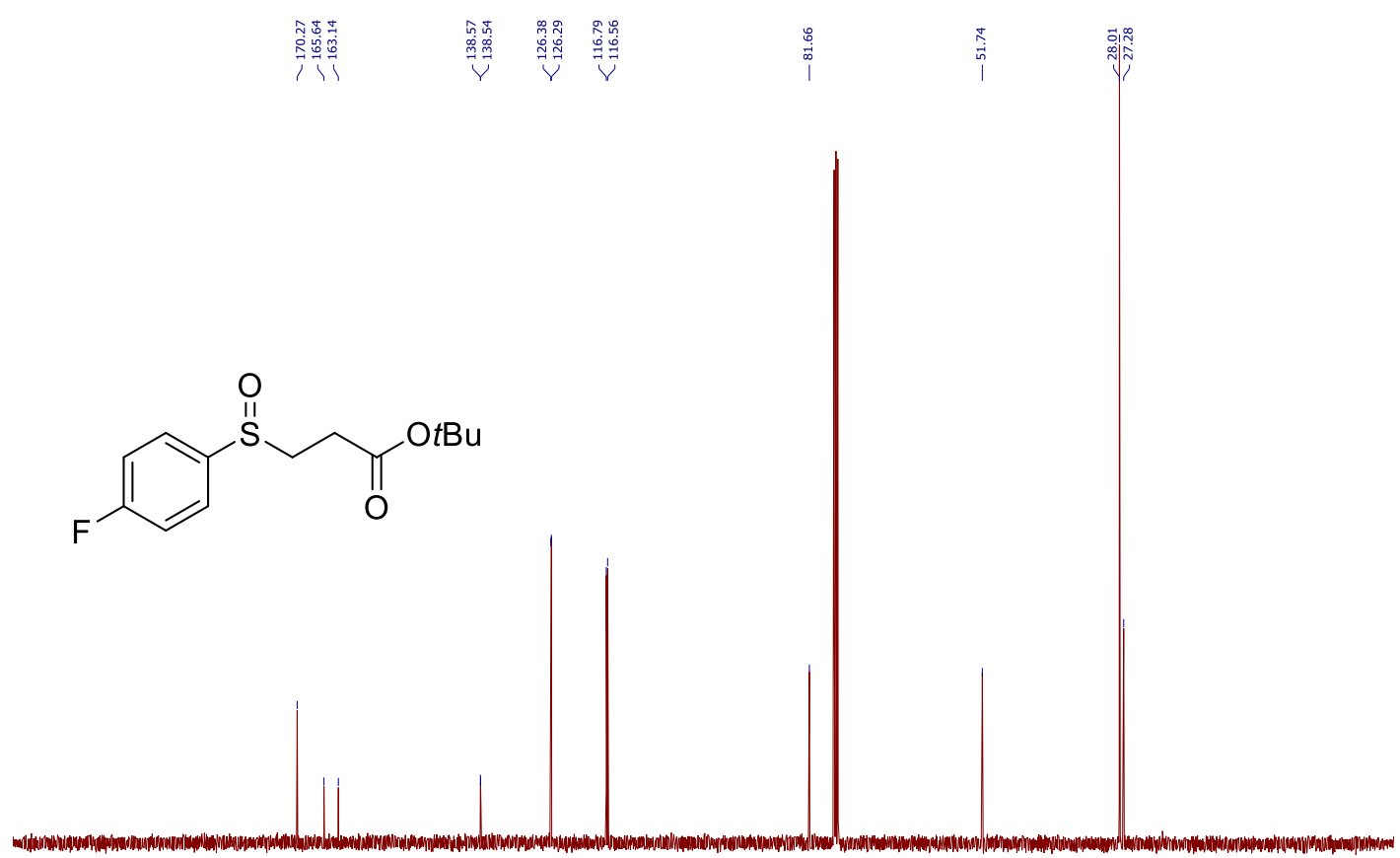

$\begin{array}{lllllllllllllllllllllll}210 & 200 & 190 & 180 & 170 & 160 & 150 & 140 & 130 & 120 & 110 & \begin{array}{c}100 \\ \mathrm{f} 1(\mathrm{ppm})\end{array} & 90 & 80 & 70 & 60 & 50 & 40 & 30 & 20 & 10 & 0 & -10\end{array}$ 
${ }^{19}$ F NMR (376 MHz, Chloroform-d)

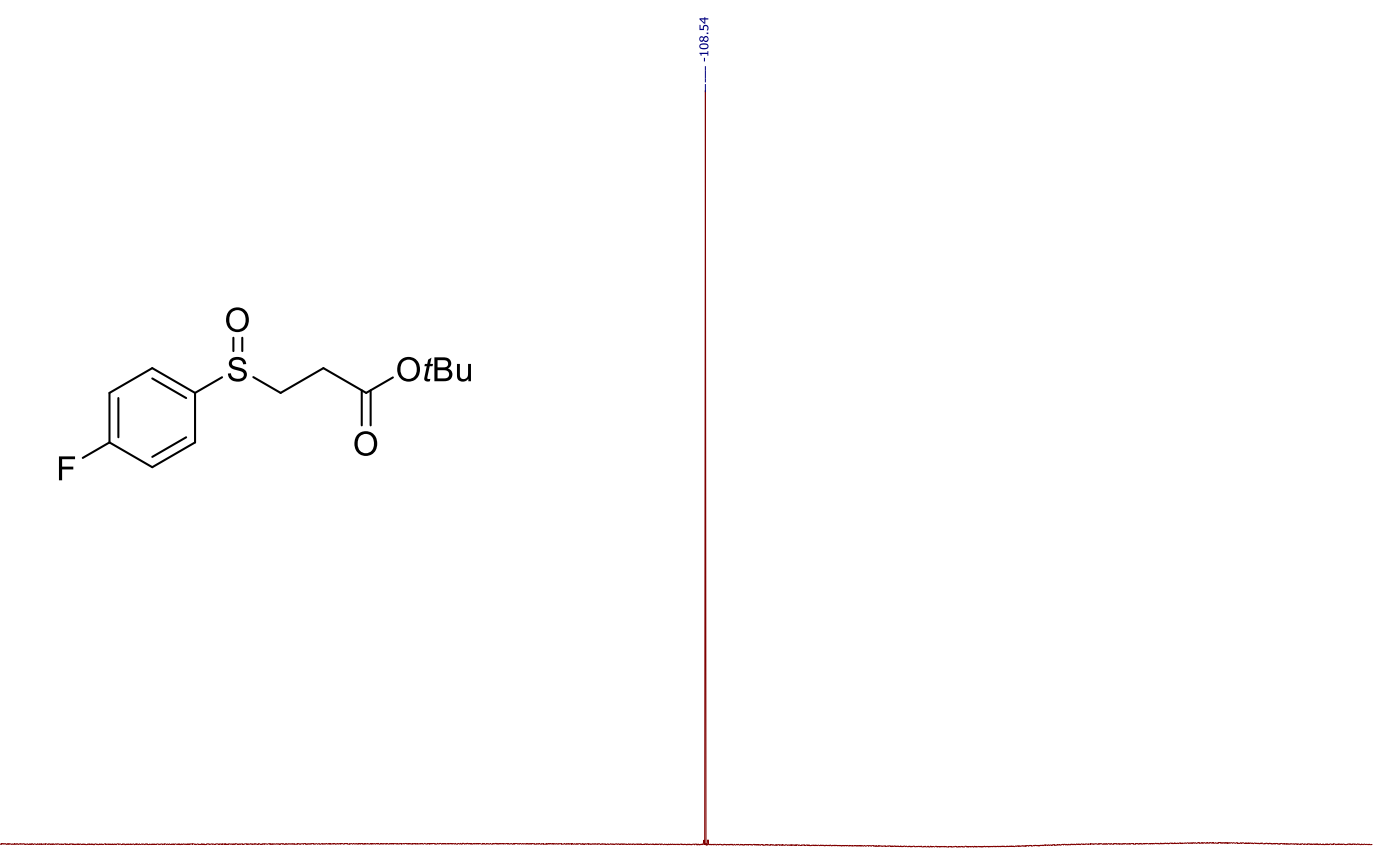

$\begin{array}{llllllllllllllllllllllll}\mid & 0 & -10 & -20 & -30 & -40 & -50 & -60 & -70 & -80 & -90 & -100 & -110 & -120 & -130 & -140 & -150 & -160 & -170 & -180 & -190 & -200 & -210 & \end{array}$




\section{Tert-butyl 3-[(4-trifluoromethylphenyl)sulfinyl]propanoate (6f)}

${ }^{1} \mathrm{H}$ NMR (400 MHz, Chloroform- $d$ )

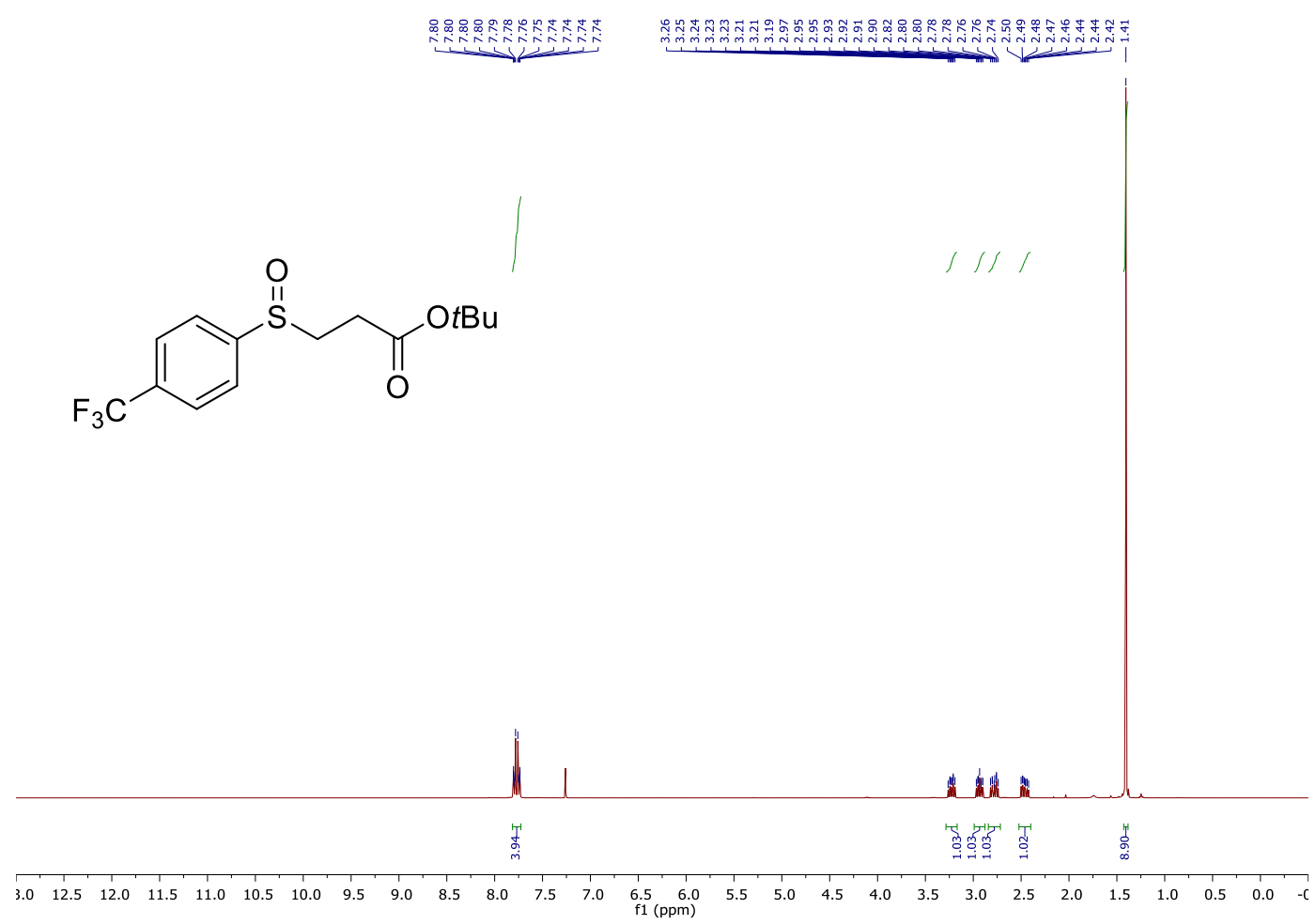

${ }^{13} \mathrm{C}$ NMR (101 MHz, Chloroform- $d$ )
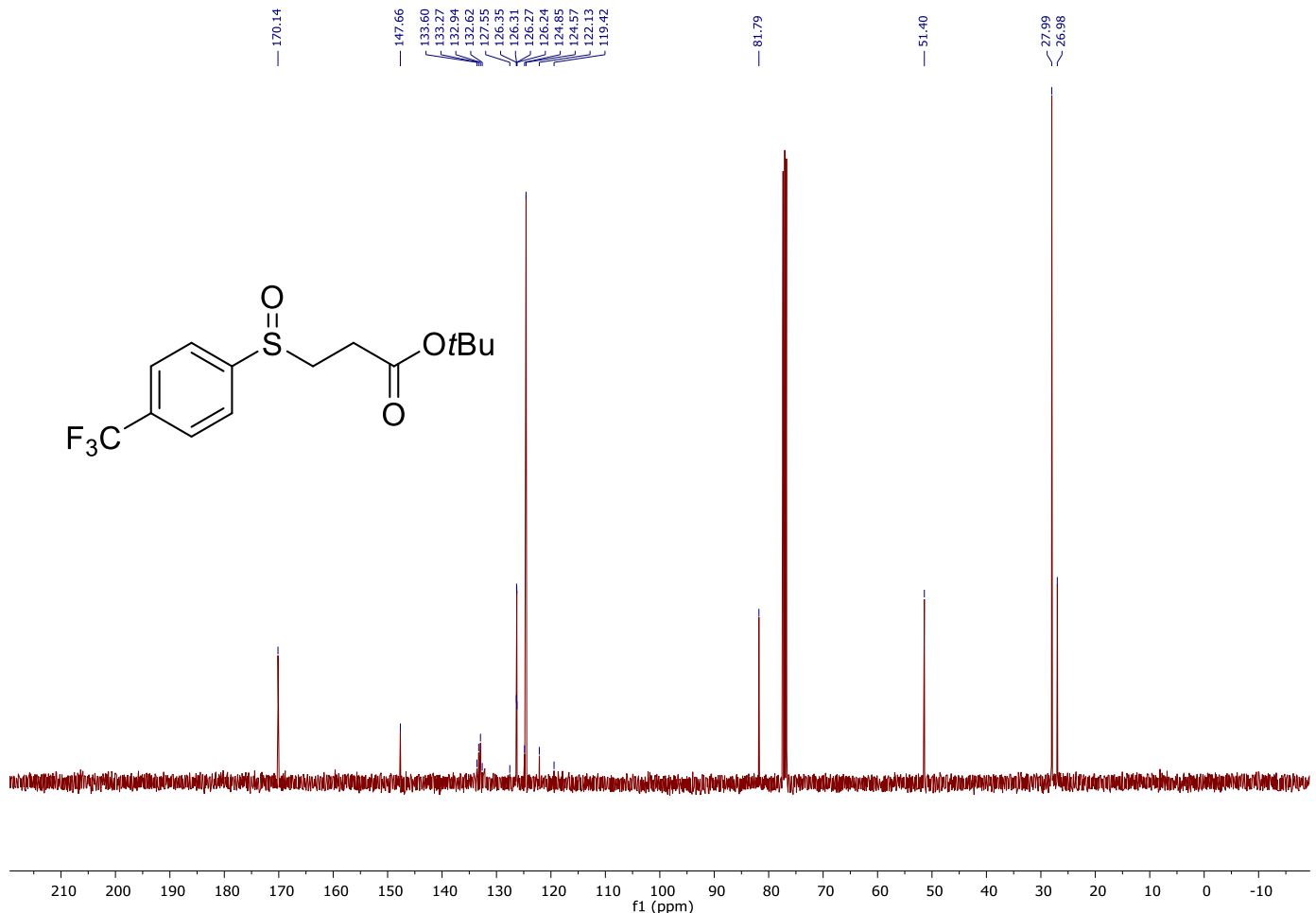
${ }^{19}$ F NMR (376 MHz, Chloroform-d)

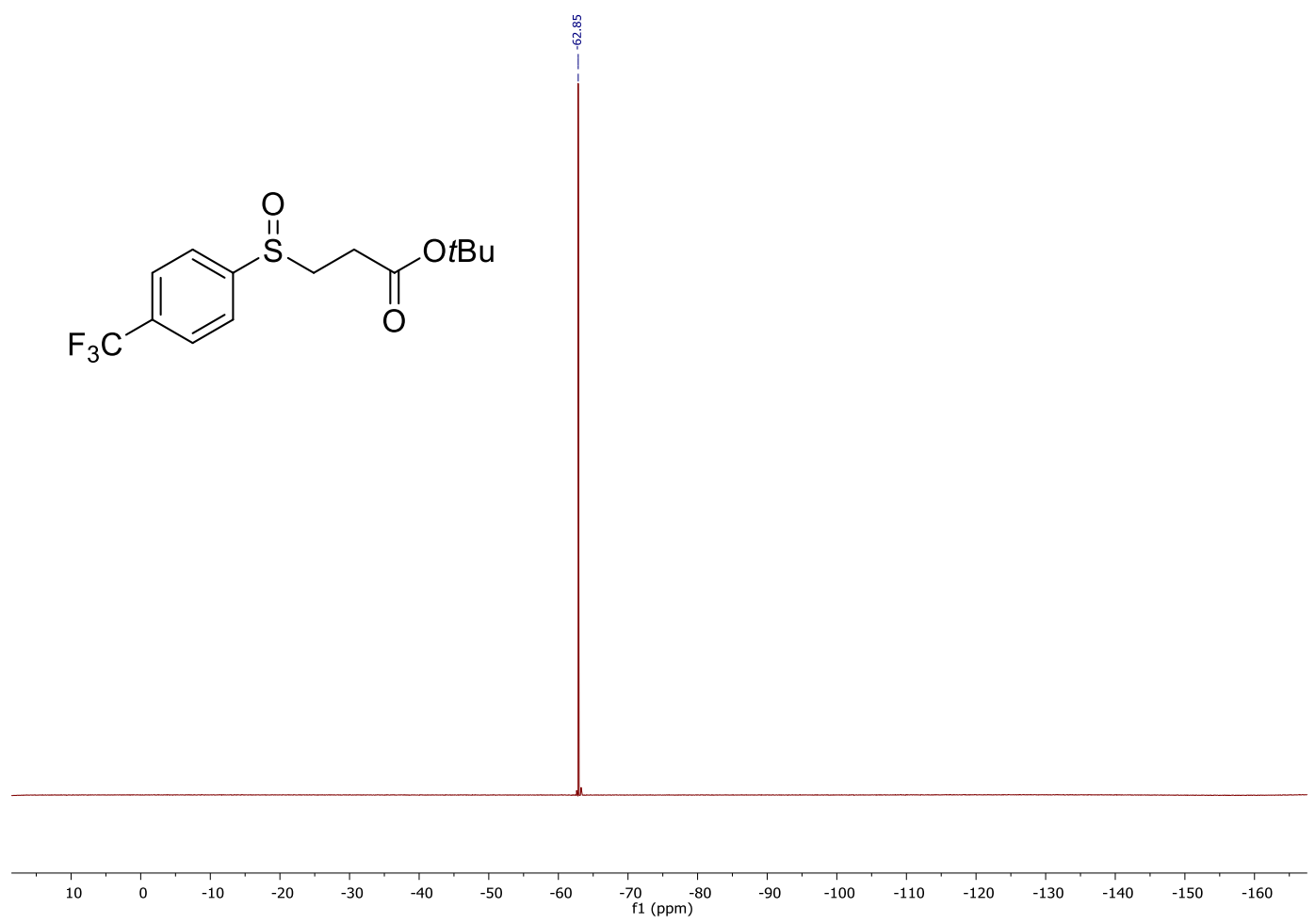




\section{Tert-butyl 3-[(4-nitrophenyl)sulfinyl]propanoate (6g)}

${ }^{1} \mathrm{H}$ NMR (400 MHz, Chloroform- $d$ )

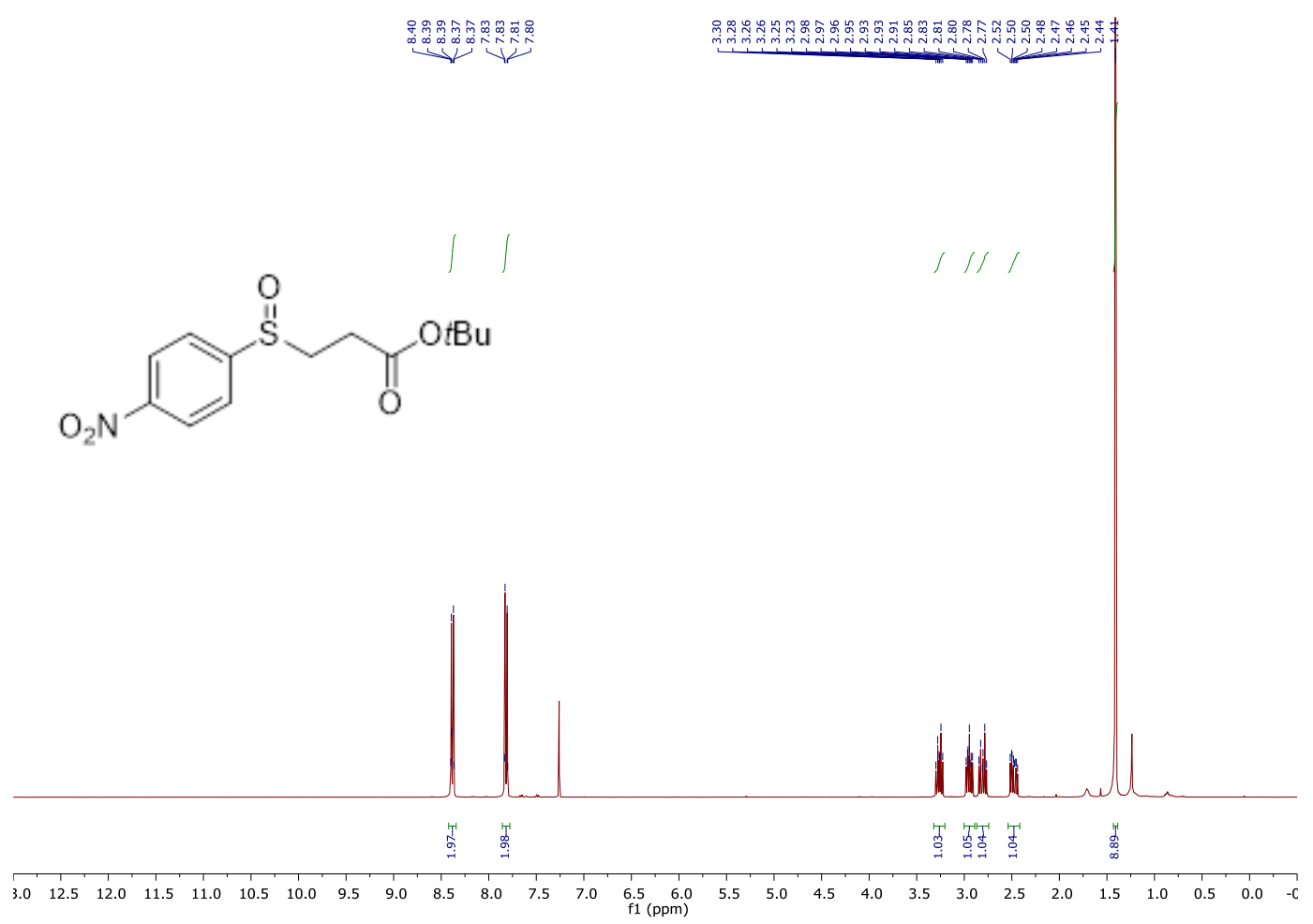

${ }^{13} \mathrm{C}$ NMR (101 MHz, Chloroform- $d$ )
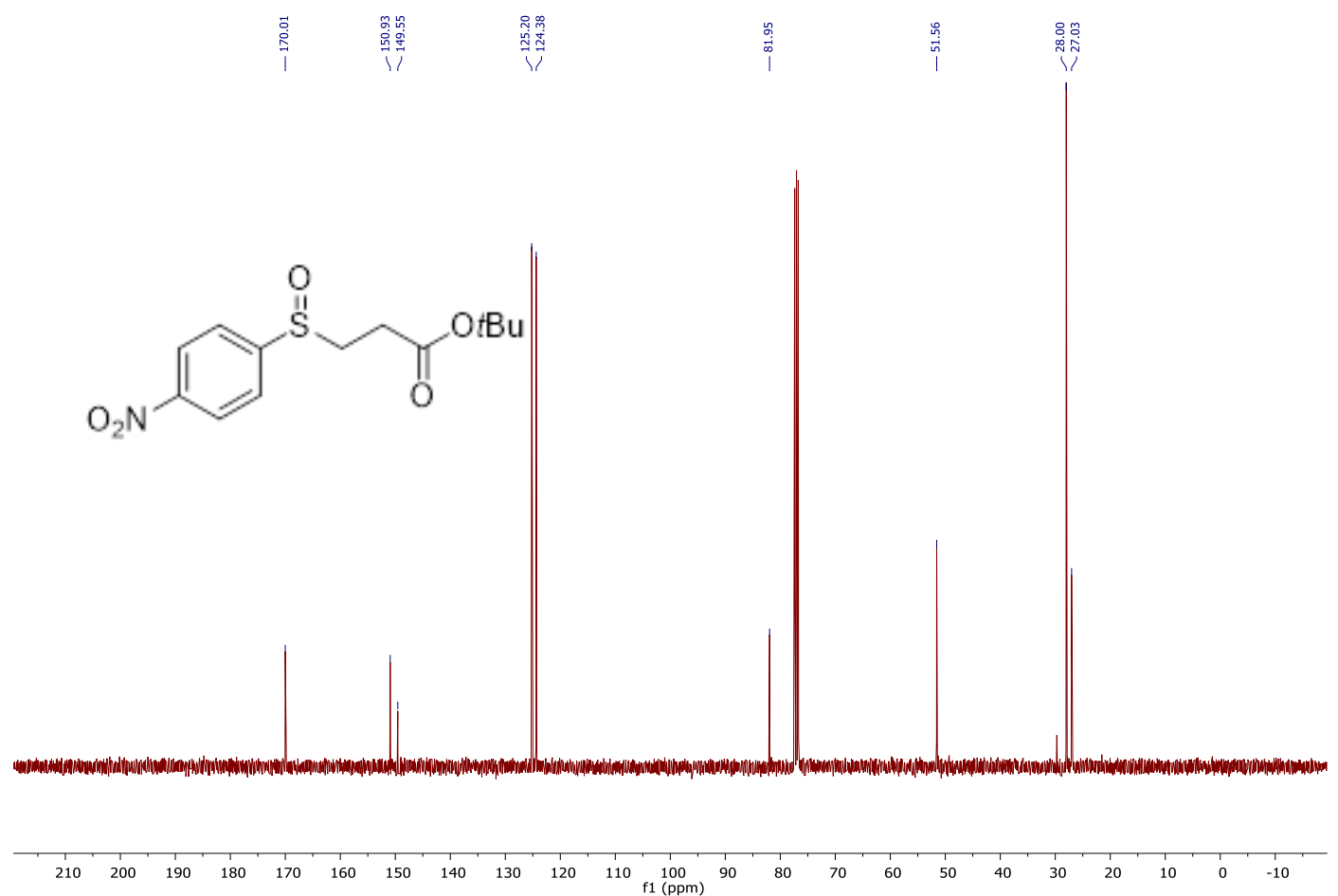


\section{Tert-butyl 3-[(2-bromophenyl)sulfinyl]propanoate (6h)}

${ }^{1} \mathrm{H}$ NMR (400 MHz, Chloroform- $d$ )

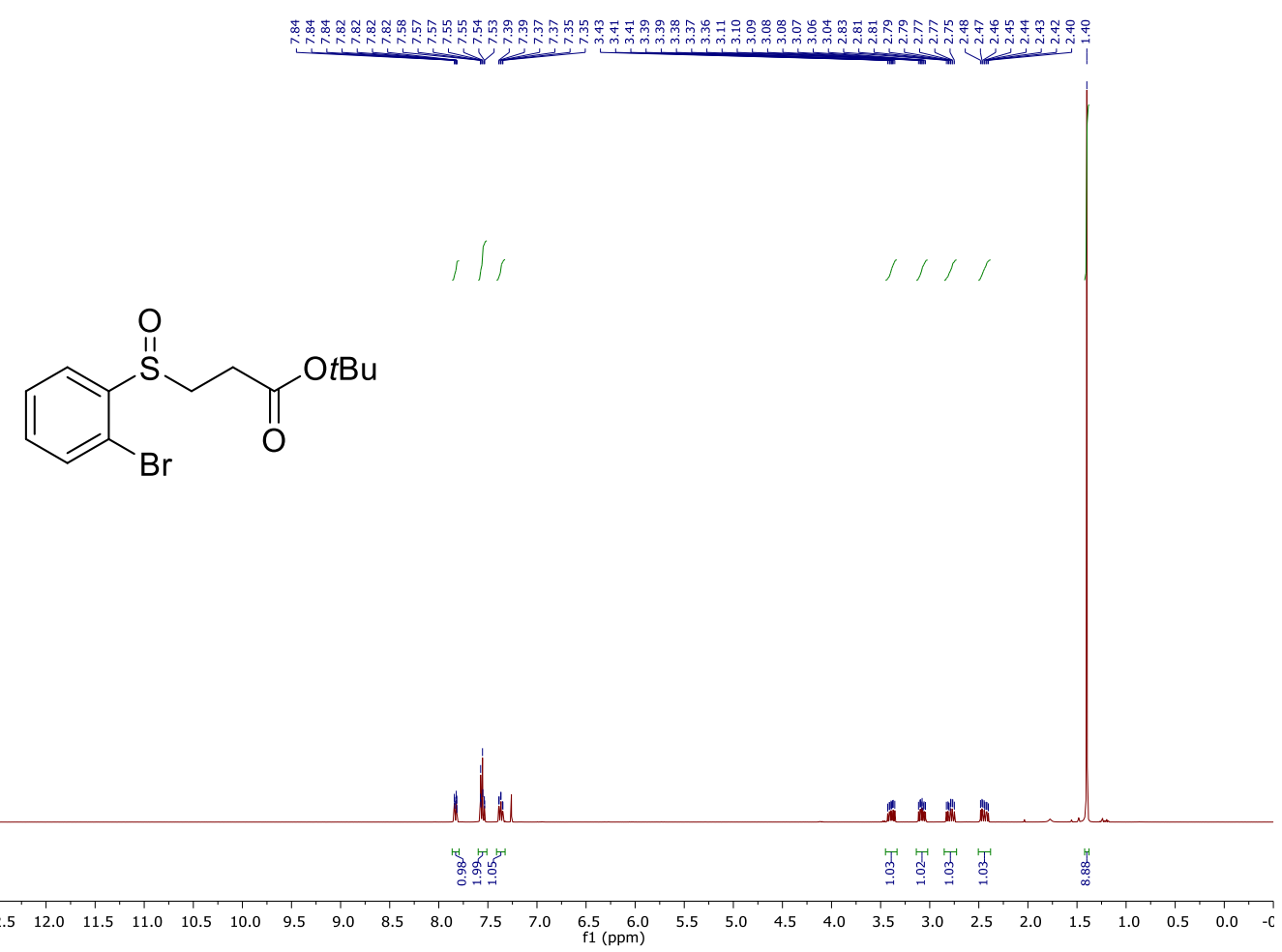

${ }^{13} \mathrm{C}$ NMR (101 MHz, Chloroform- $d$ )

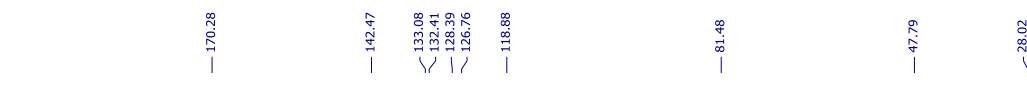<smiles>CCCCCC(=O)OCCS(=O)c1ccccc1Br</smiles>

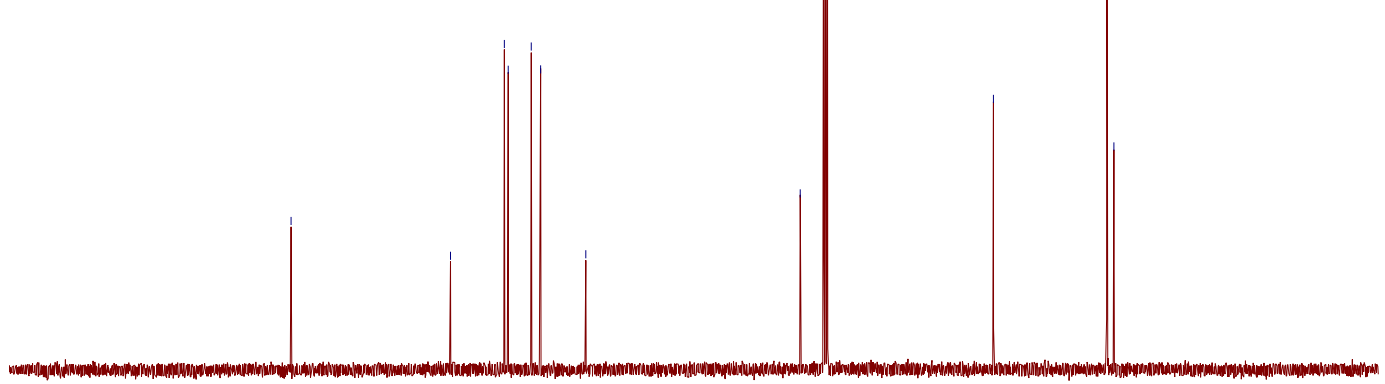

$\begin{array}{llllllllllll}210 & 200 & 190 & 180 & 170 & 160 & 150 & 140 & 130 & 120 & 110 & 100 \\ \mathrm{f} 1(\mathrm{ppm}) & 90 & 80\end{array}$ 


\section{Tert-butyl 3-[(3-methylphenyl)sulfinyl]propanoate (6i)}

${ }^{1} \mathrm{H}$ NMR (400 MHz, Chloroform- $d$ )

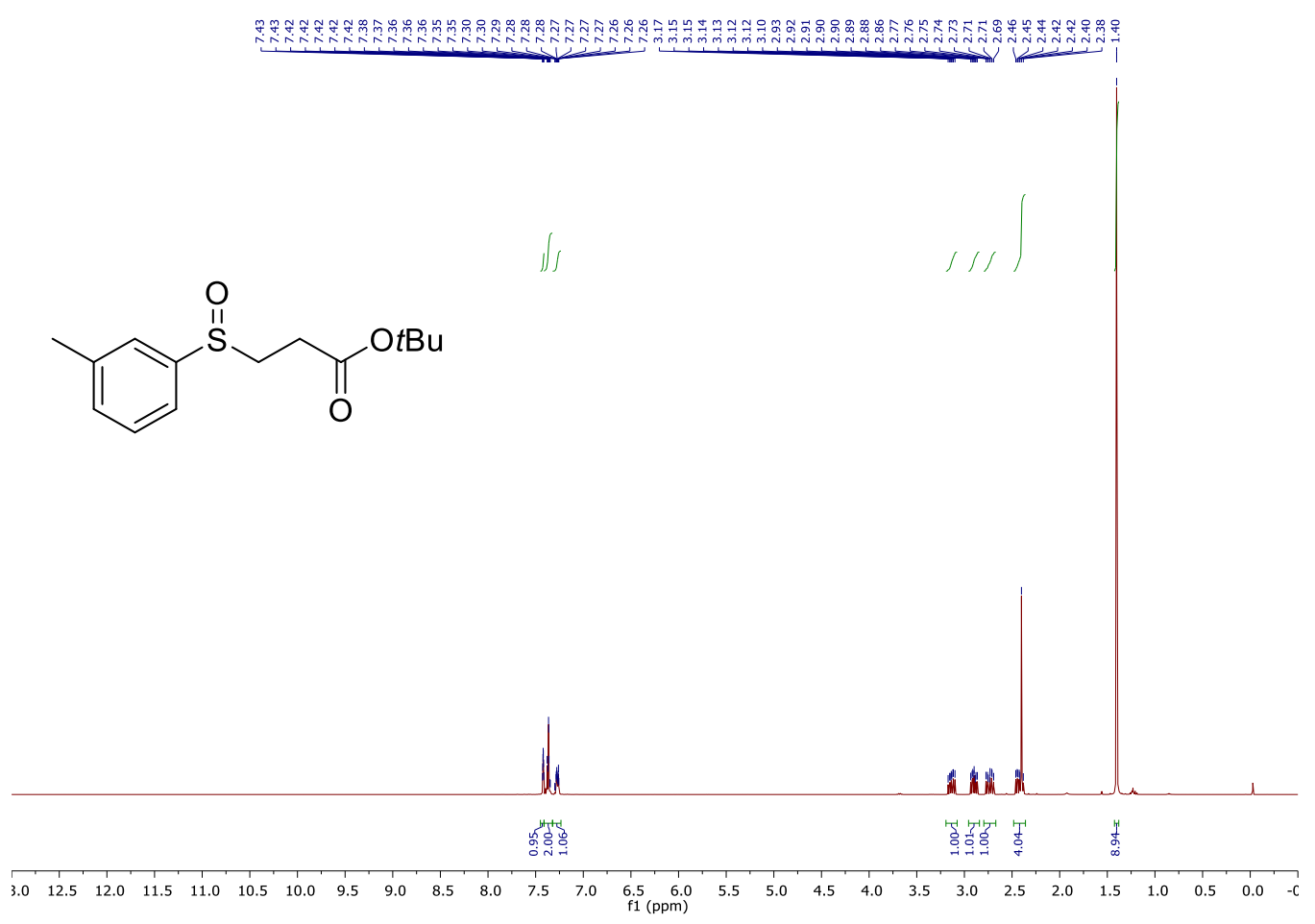

${ }^{13} \mathrm{C}$ NMR (101 MHz, Chloroform- $d$ )

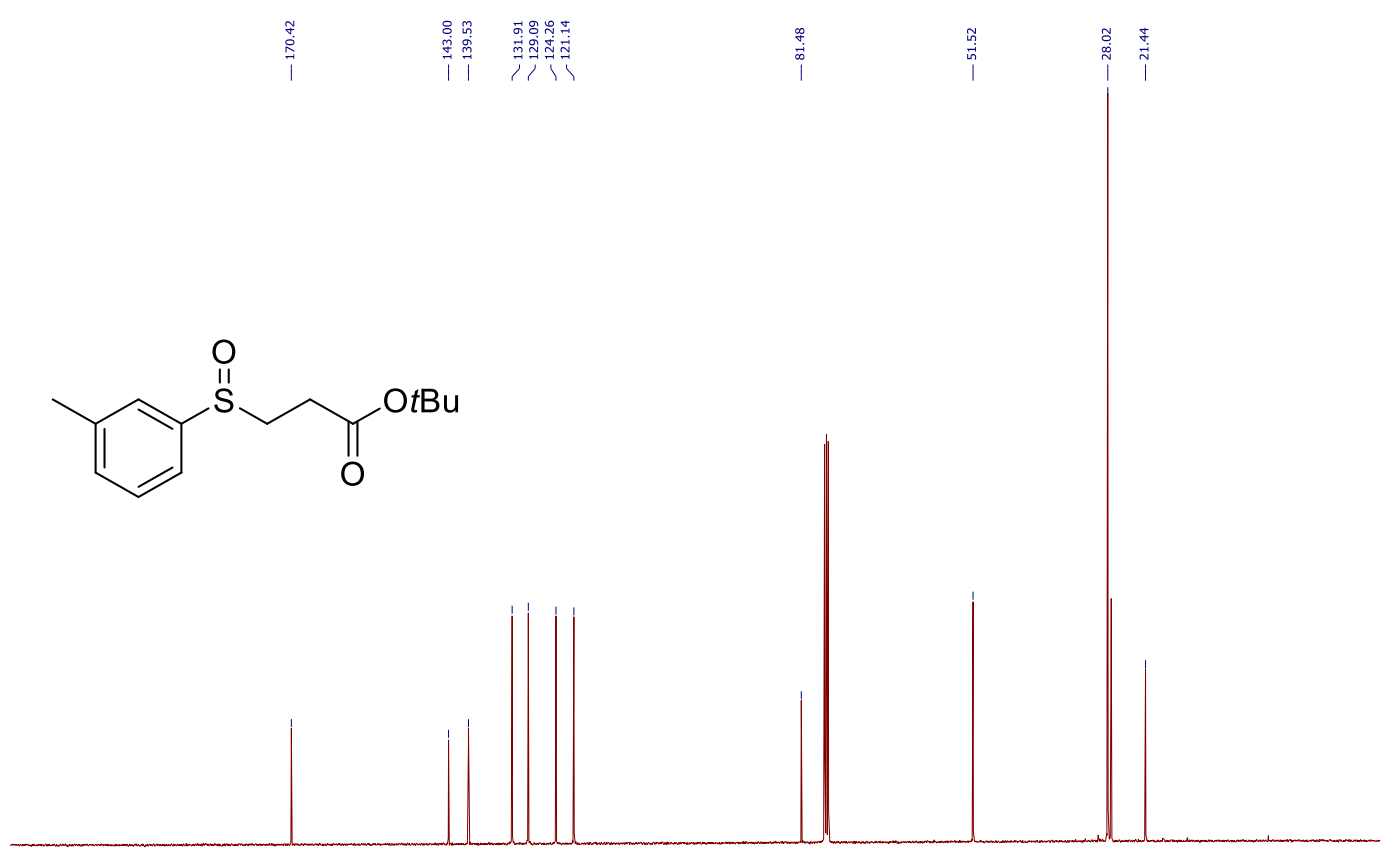

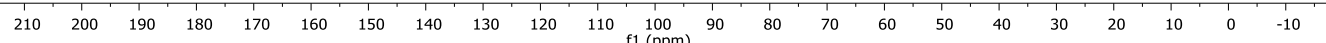




\section{Tert-butyl 3-[(3-bromophenyl)sulfinyl]propanoate (6j)}

${ }^{1} \mathrm{H}$ NMR (400 MHz, Chloroform- $d$ )

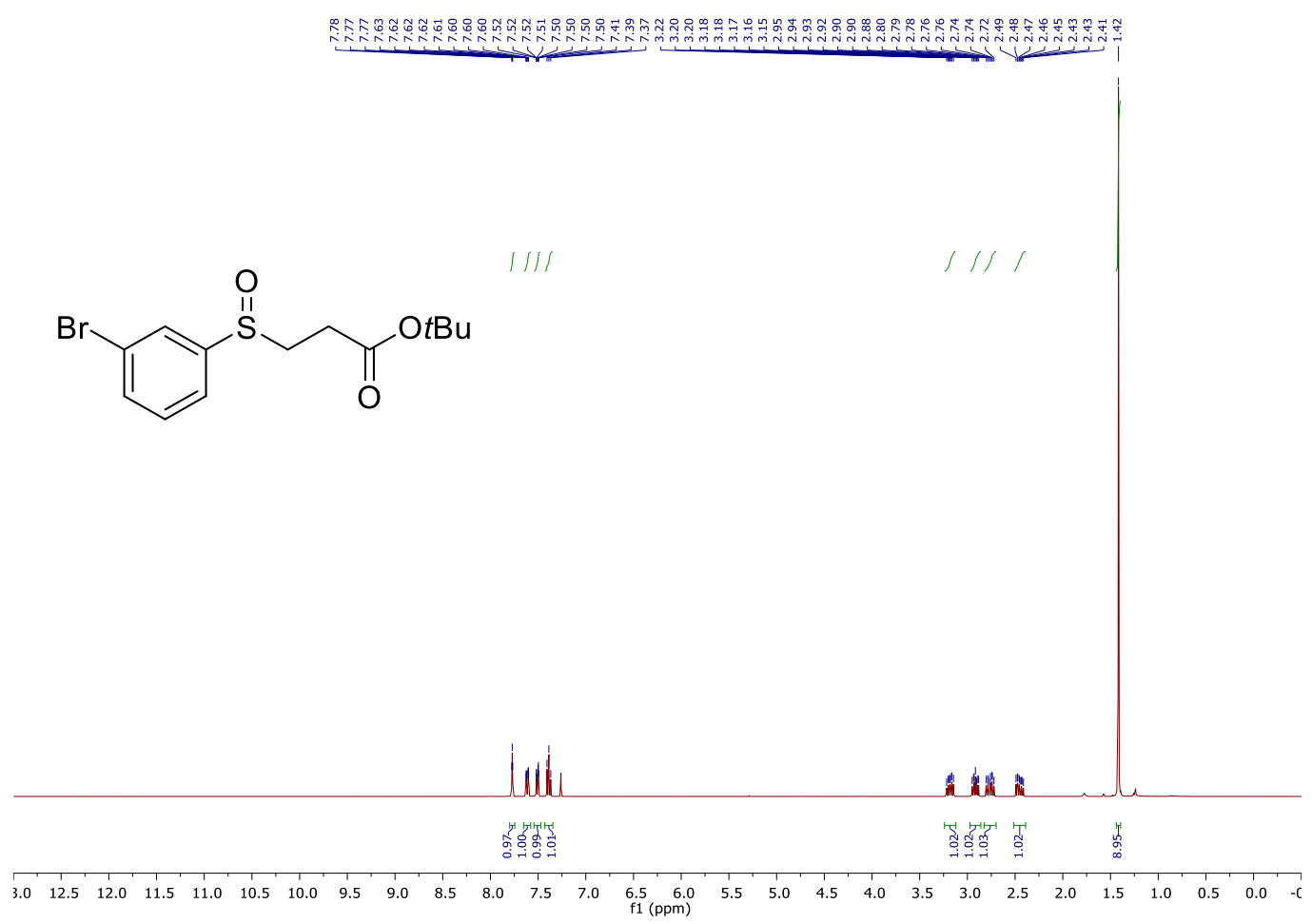

${ }^{13} \mathrm{C}$ NMR (101 MHz, Chloroform- $d$ )
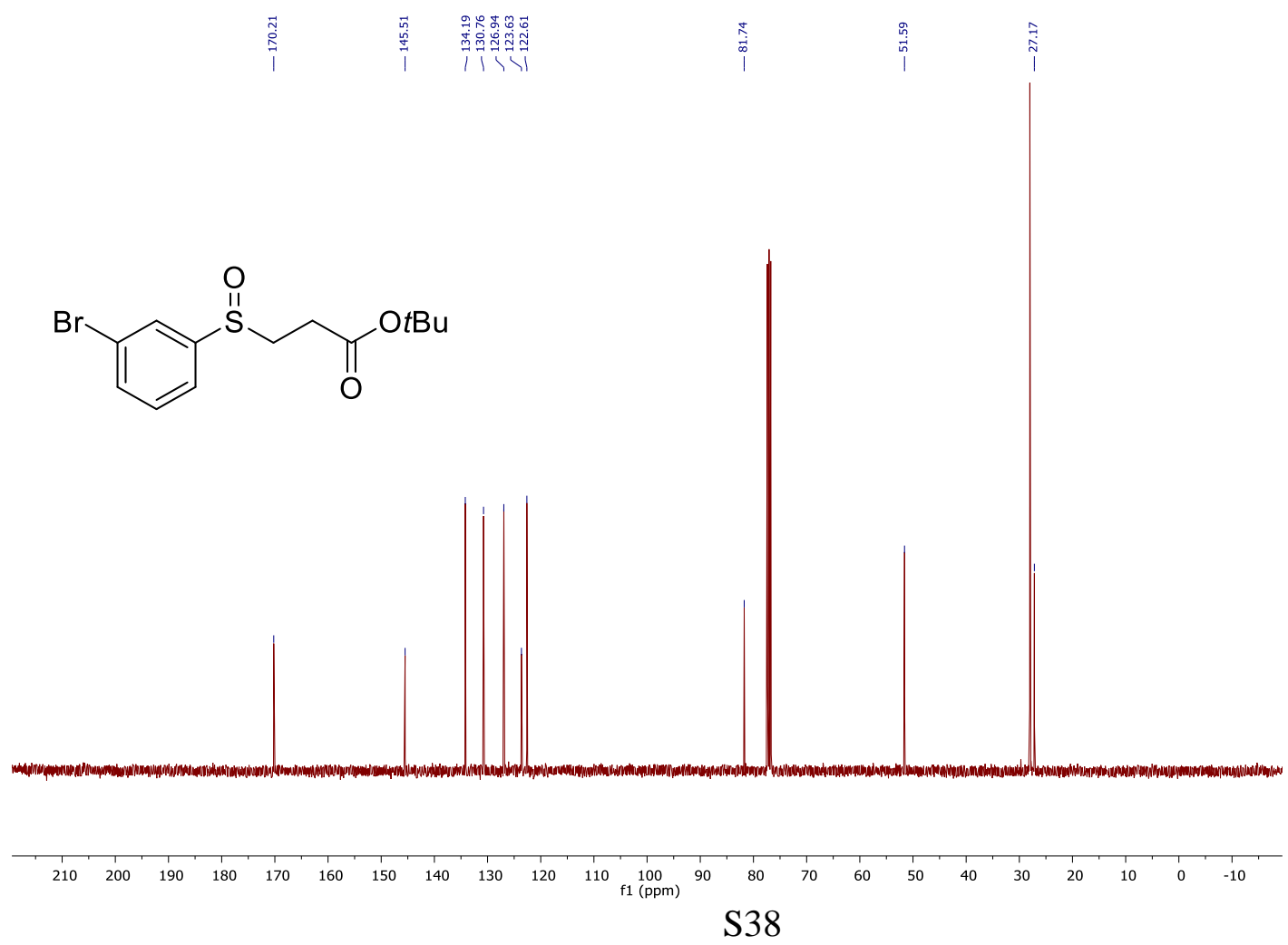


\section{Tert-butyl 3-((pyridin-2-yl)sulfinyl)propanoate (6k)}

${ }^{1} \mathrm{H}$ NMR (400 MHz, Chloroform- $d$ )

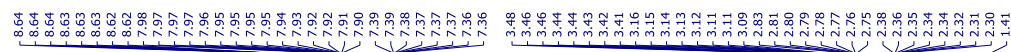<smiles>CCCOC(=O)CCS(=O)c1ccccn1</smiles>

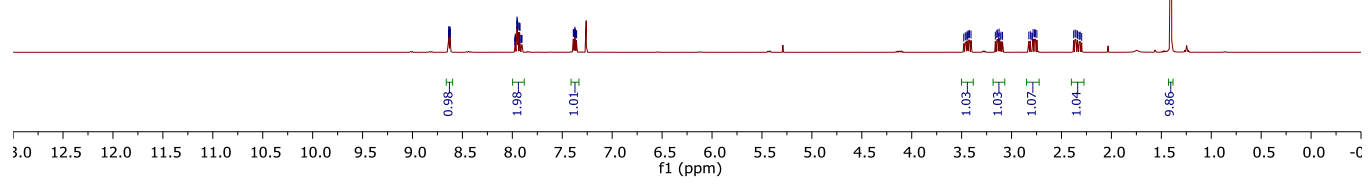

${ }^{13} \mathrm{C}$ NMR (101 MHz, Chloroform- $d$ )

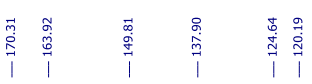<smiles>CCCCOC(=O)CCS(=O)c1ccccn1</smiles>
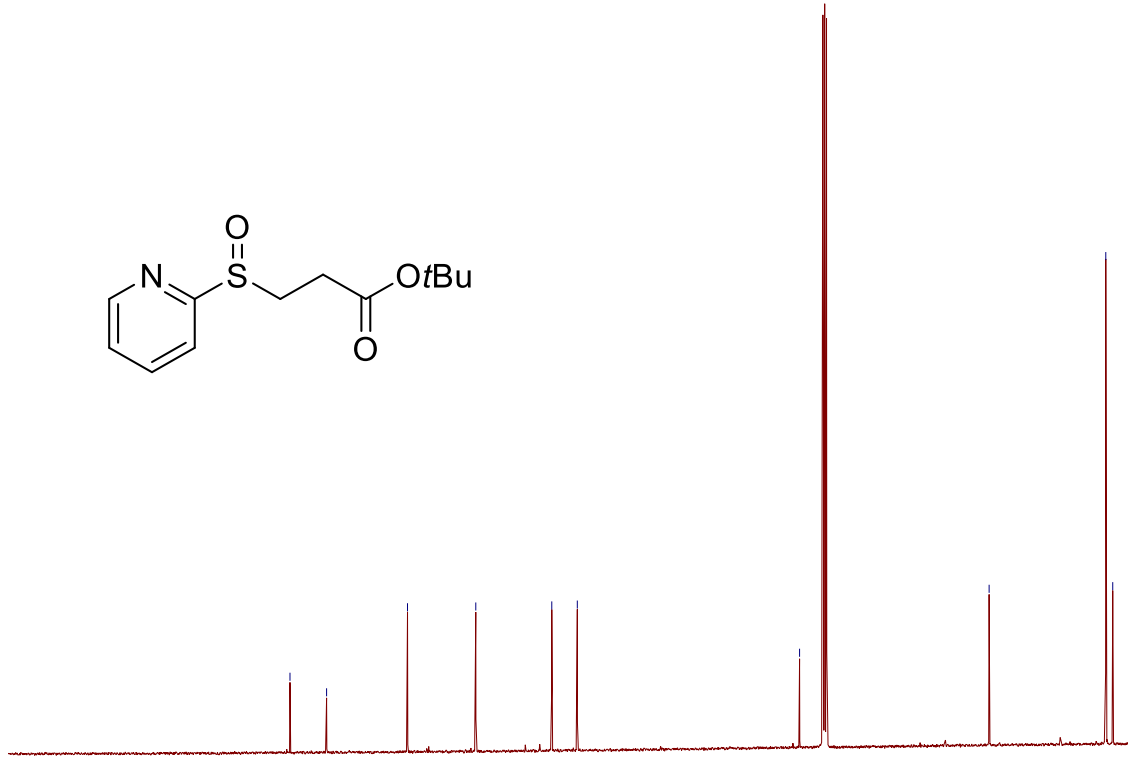


\section{Tert-butyl 3-((thiophen-2-yl)sulfinyl)propanoate (6l)}

${ }^{1} \mathrm{H}$ NMR (400 MHz, Chloroform- $d$ )

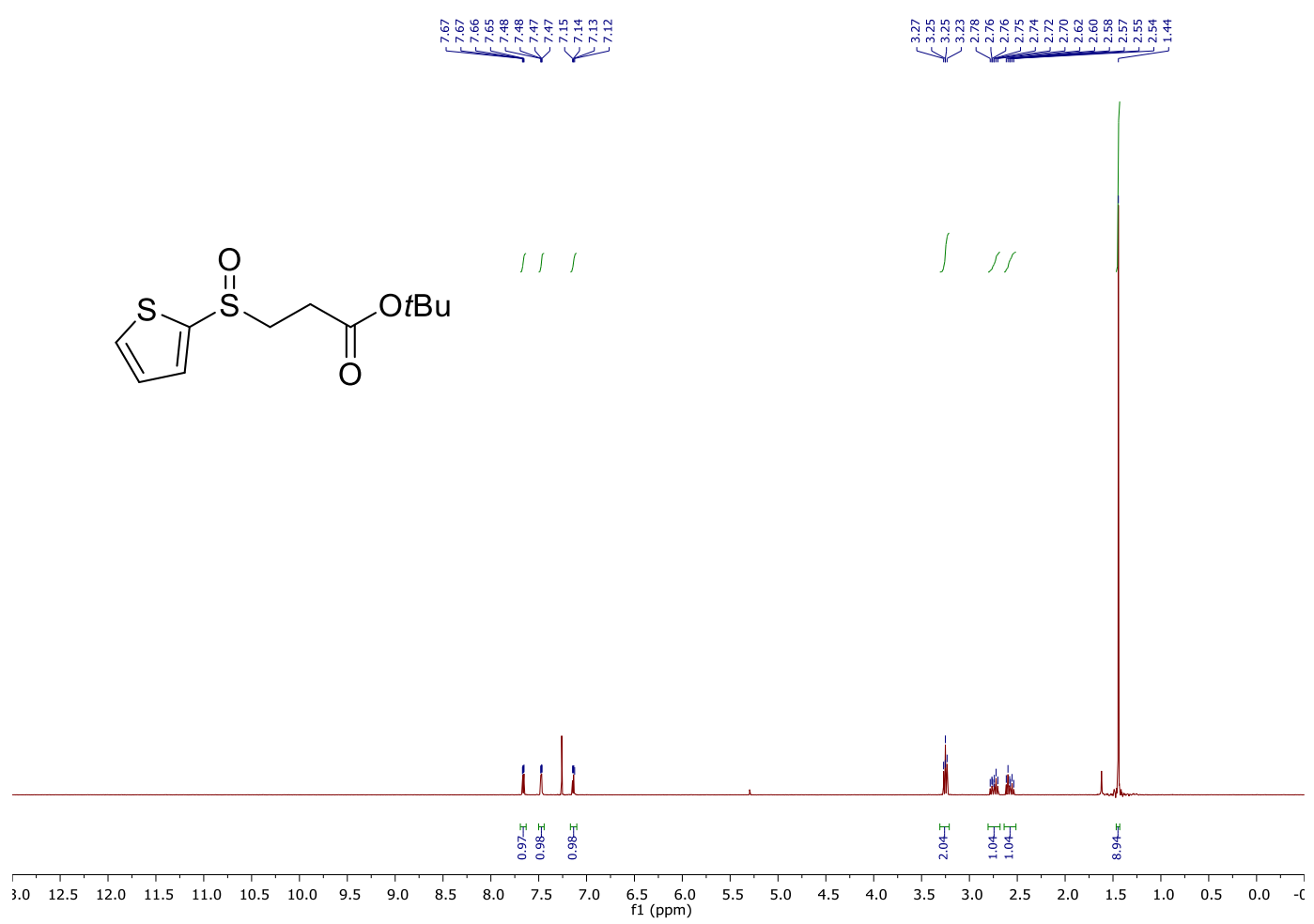

${ }^{13} \mathrm{C}$ NMR (101 MHz, Chloroform- $d$ )
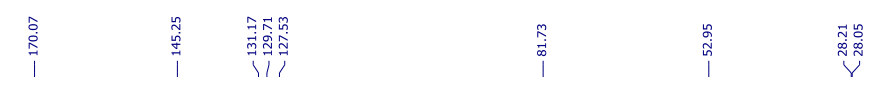<smiles>CCCCOC(=O)CCS(=O)c1cccs1</smiles>

$\begin{array}{llllllllllll}210 & 200 & 190 & 180 & 170 & 160 & 150 & 140 & 130 & 120 & 110 & 100 \\ \mathrm{f} 1(\mathrm{ppm}) & 90\end{array}$ 


\section{Tert-butyl 3-((2-methylfruna-3-yl)sulfinyl)propanoate (6m)}

${ }^{1} \mathrm{H}$ NMR (400 MHz, Chloroform- $d$ )

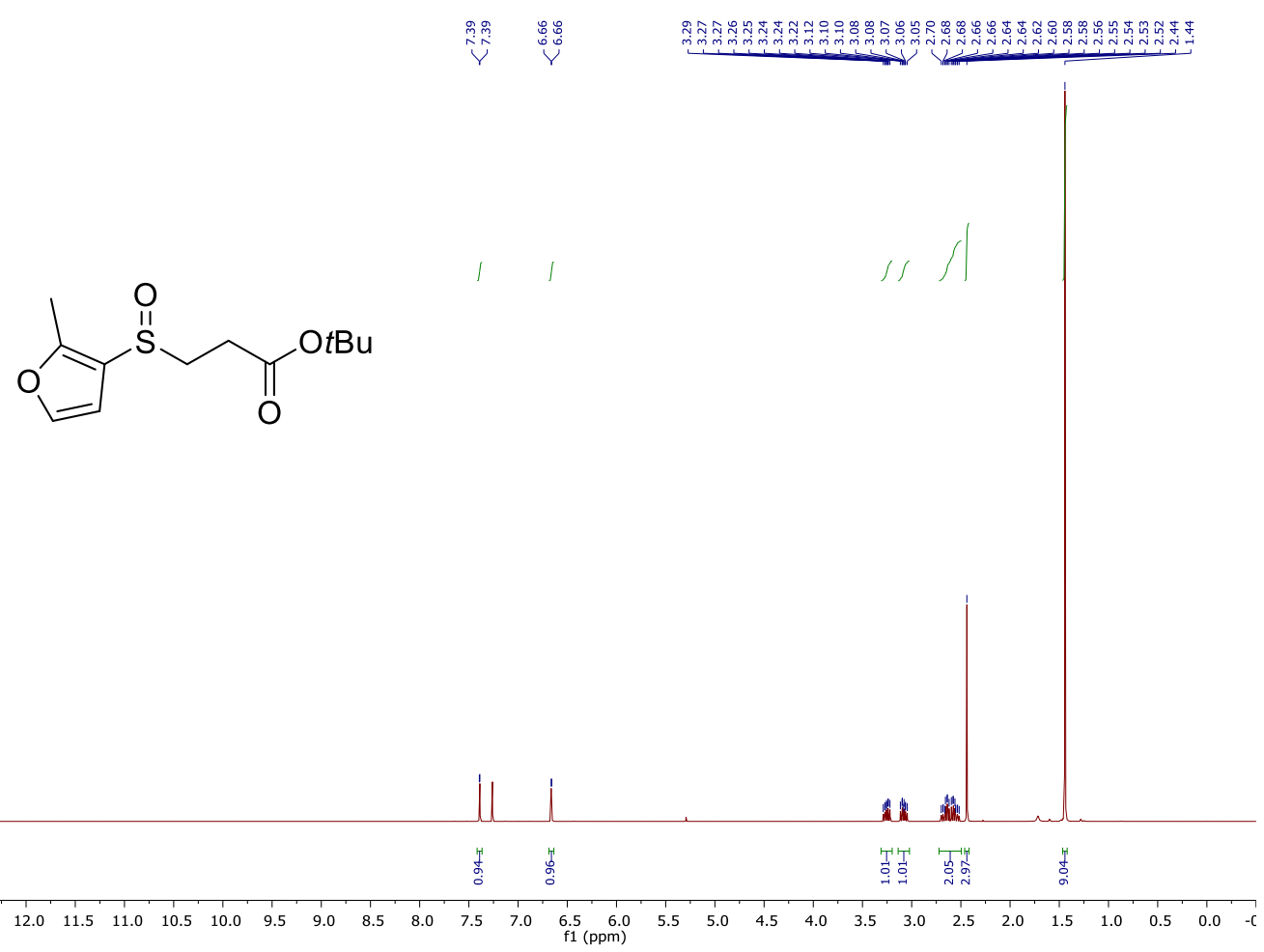

${ }^{13} \mathrm{C}$ NMR (101 MHz, Chloroform- $d$ )

$$
\underbrace{O t B u}_{0}
$$<smiles>CCCCCC(=O)OCCS(=O)c1ccoc1C</smiles> 


\section{Tert-butyl 3-(pentylsulfinyl)propanoate (6n)}

${ }^{1} \mathrm{H}$ NMR (400 MHz, Chloroform- $d$ )

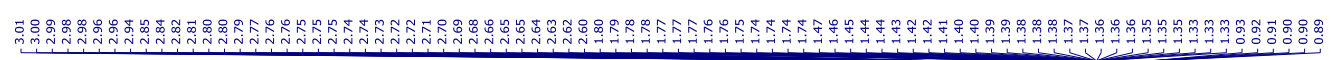
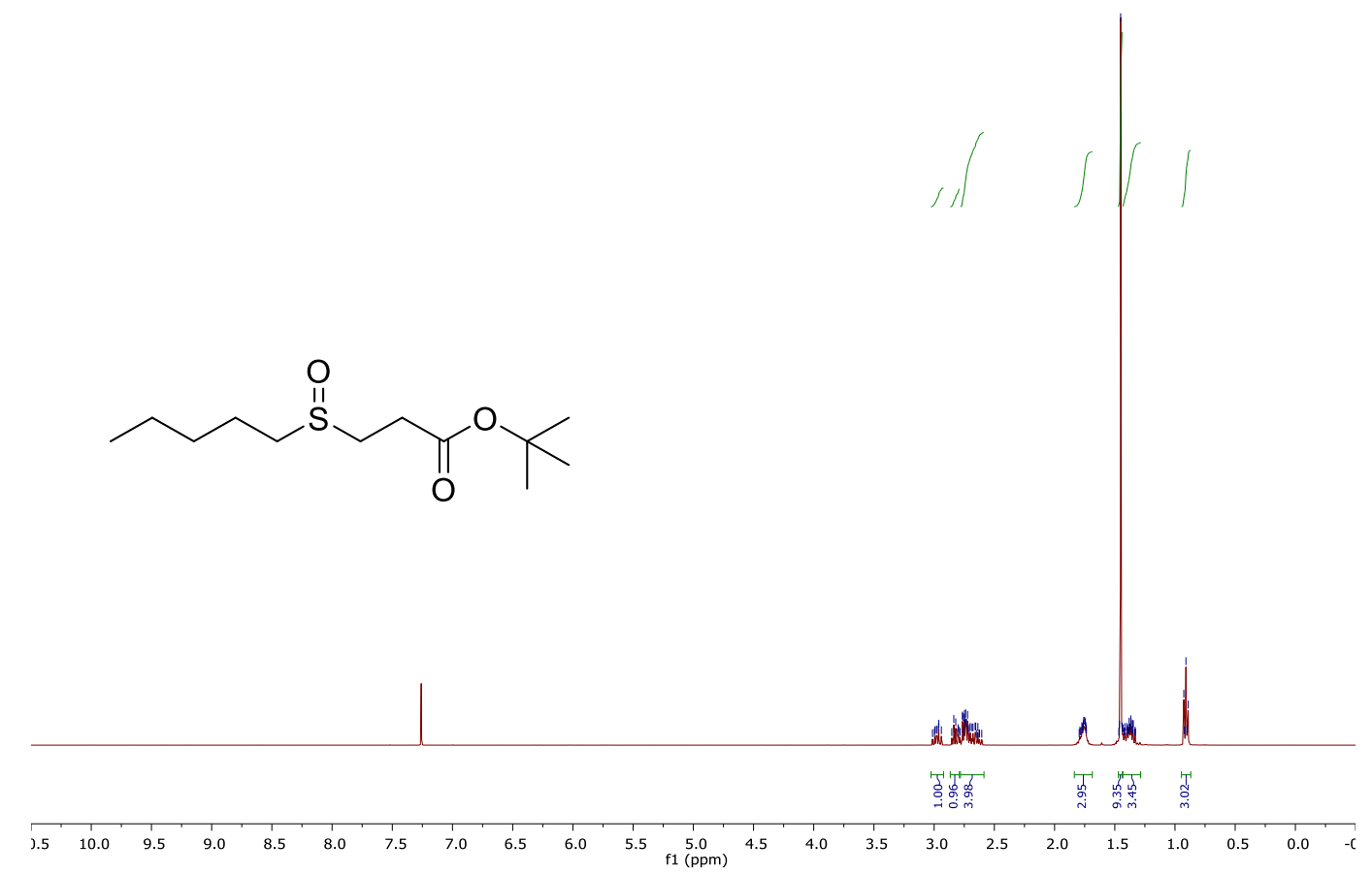

${ }^{13} \mathrm{C}$ NMR (101 MHz, Chloroform- $d$ )
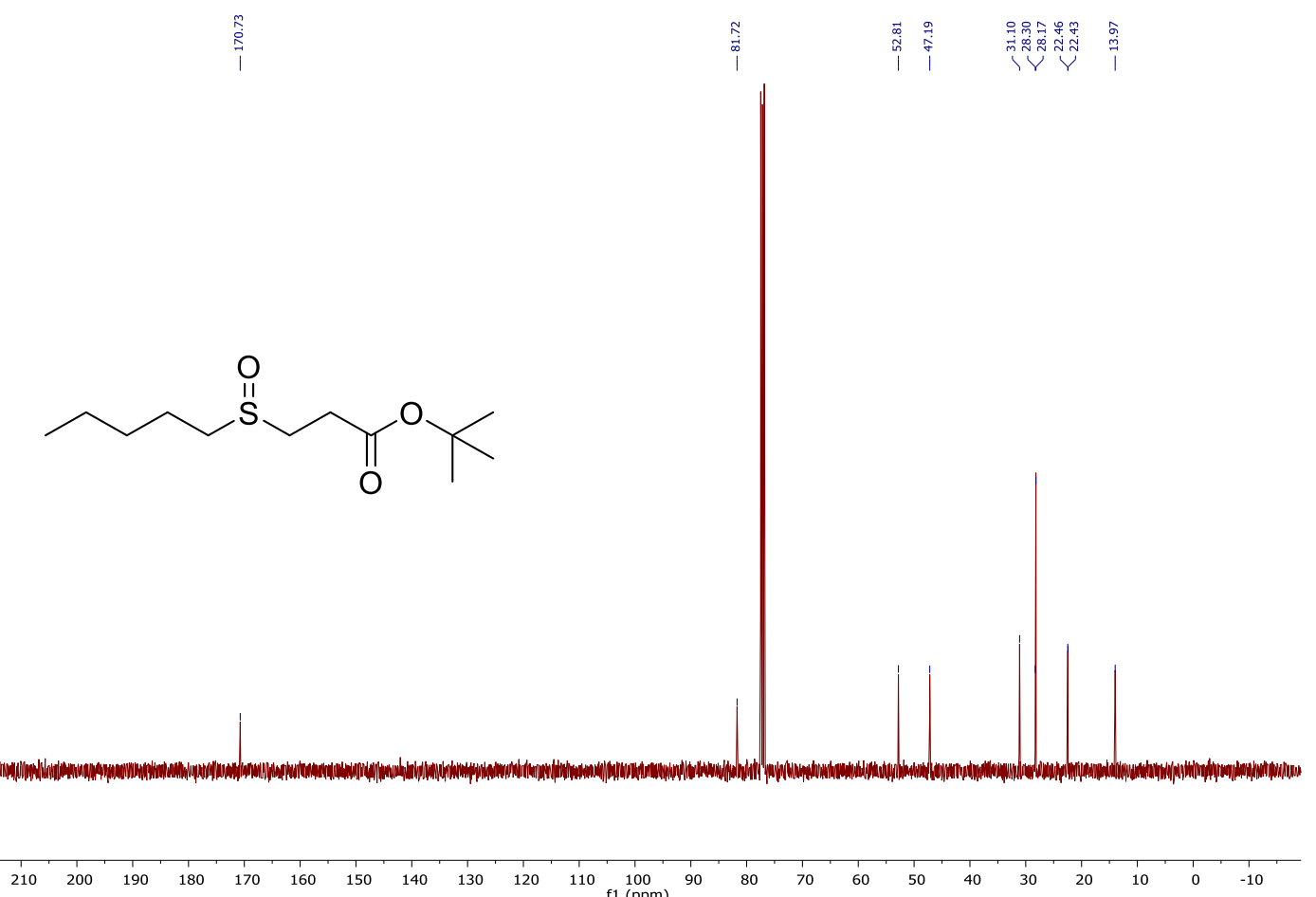


\section{Tert-butyl 3-(cyclohexylsulfinyl)propanoate (6o)}

${ }^{1} \mathrm{H}$ NMR (400 MHz, Chloroform- $d$ )

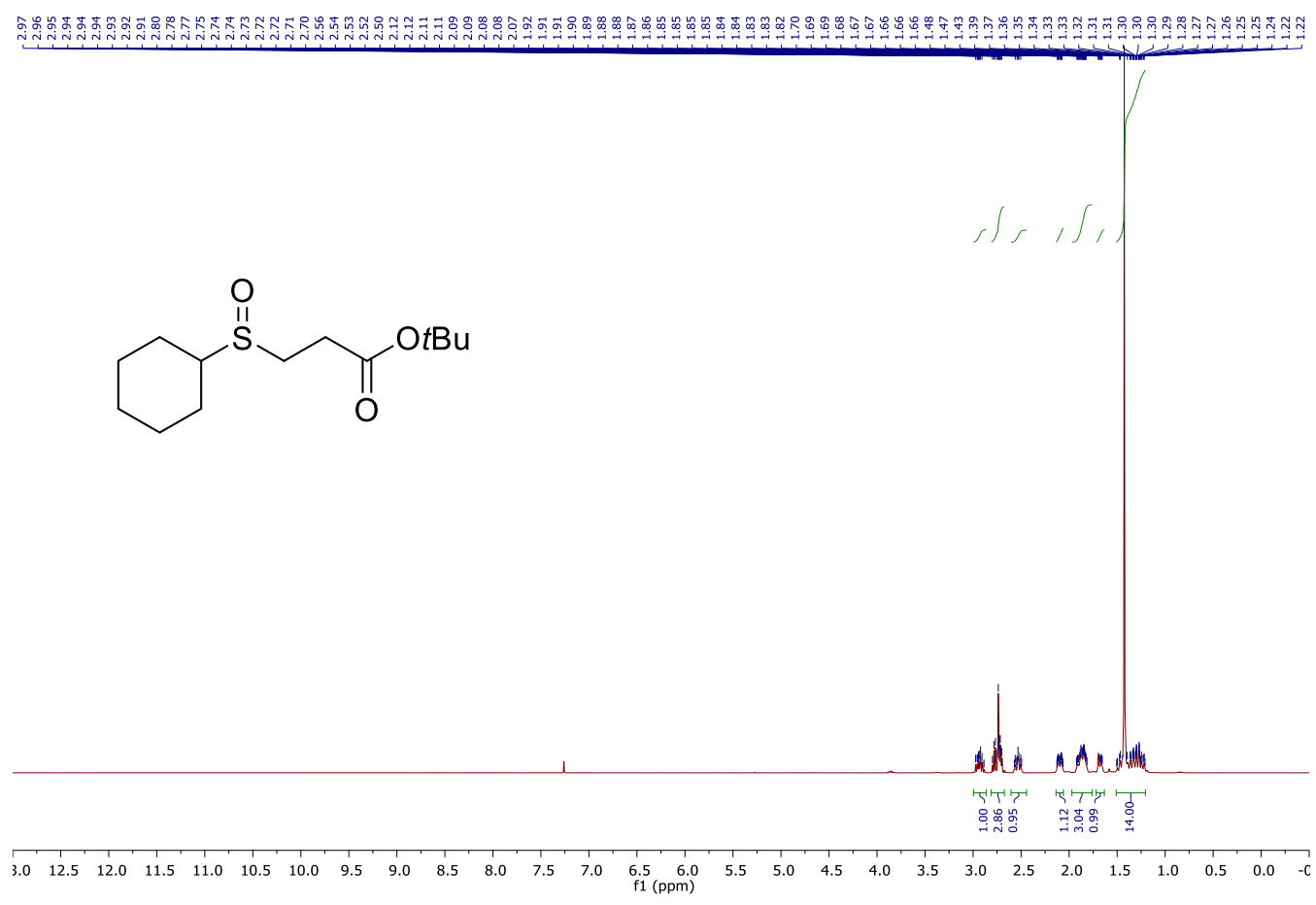

${ }^{13} \mathrm{C}$ NMR (101 MHz, Chloroform- $d$ )
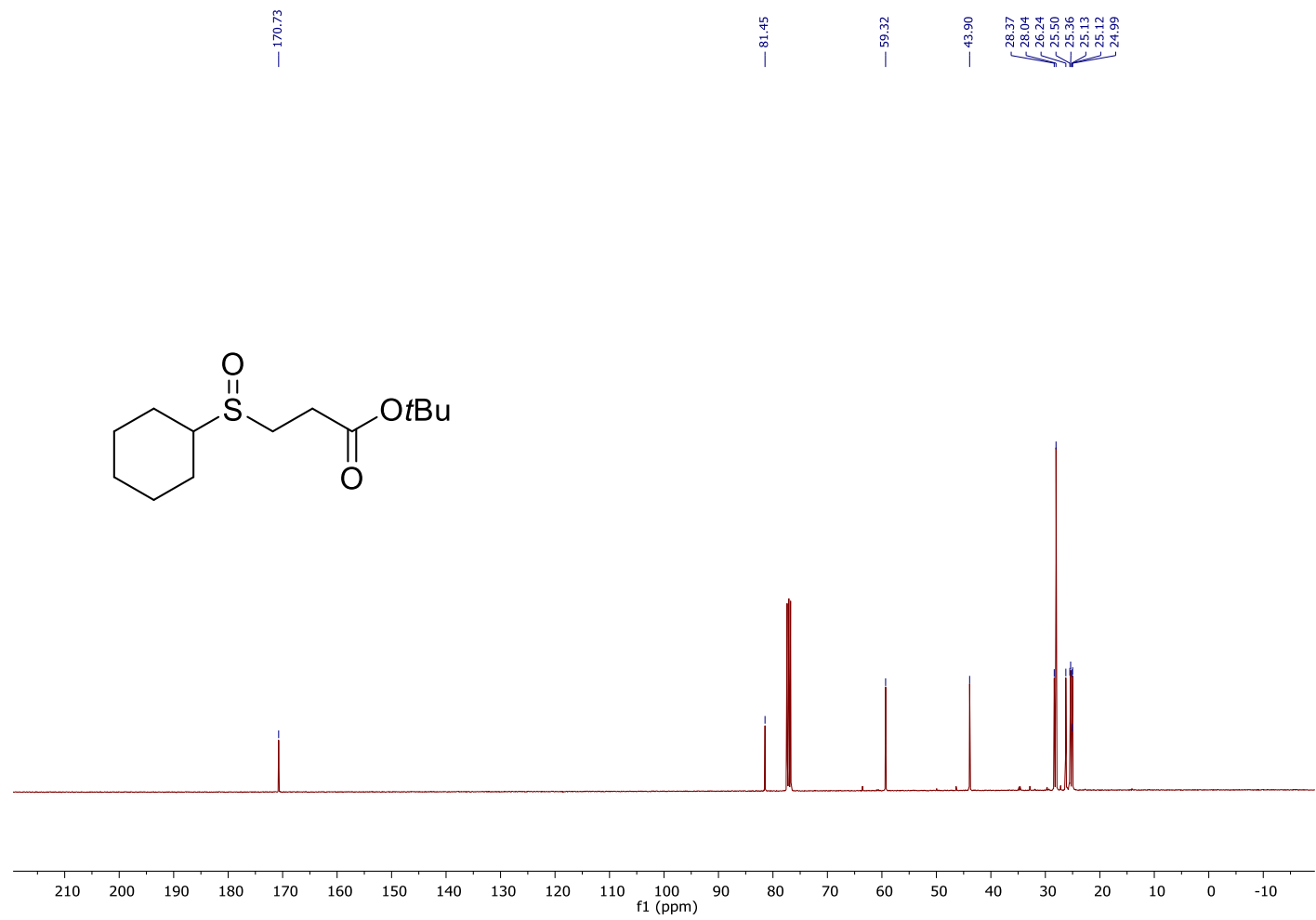


\section{Triisopropyl((4-methylphenylsulfinyl)ethynyl)silane (10a)}

${ }^{1} \mathrm{H}$ NMR (400 MHz, Chloroform- $d$ )

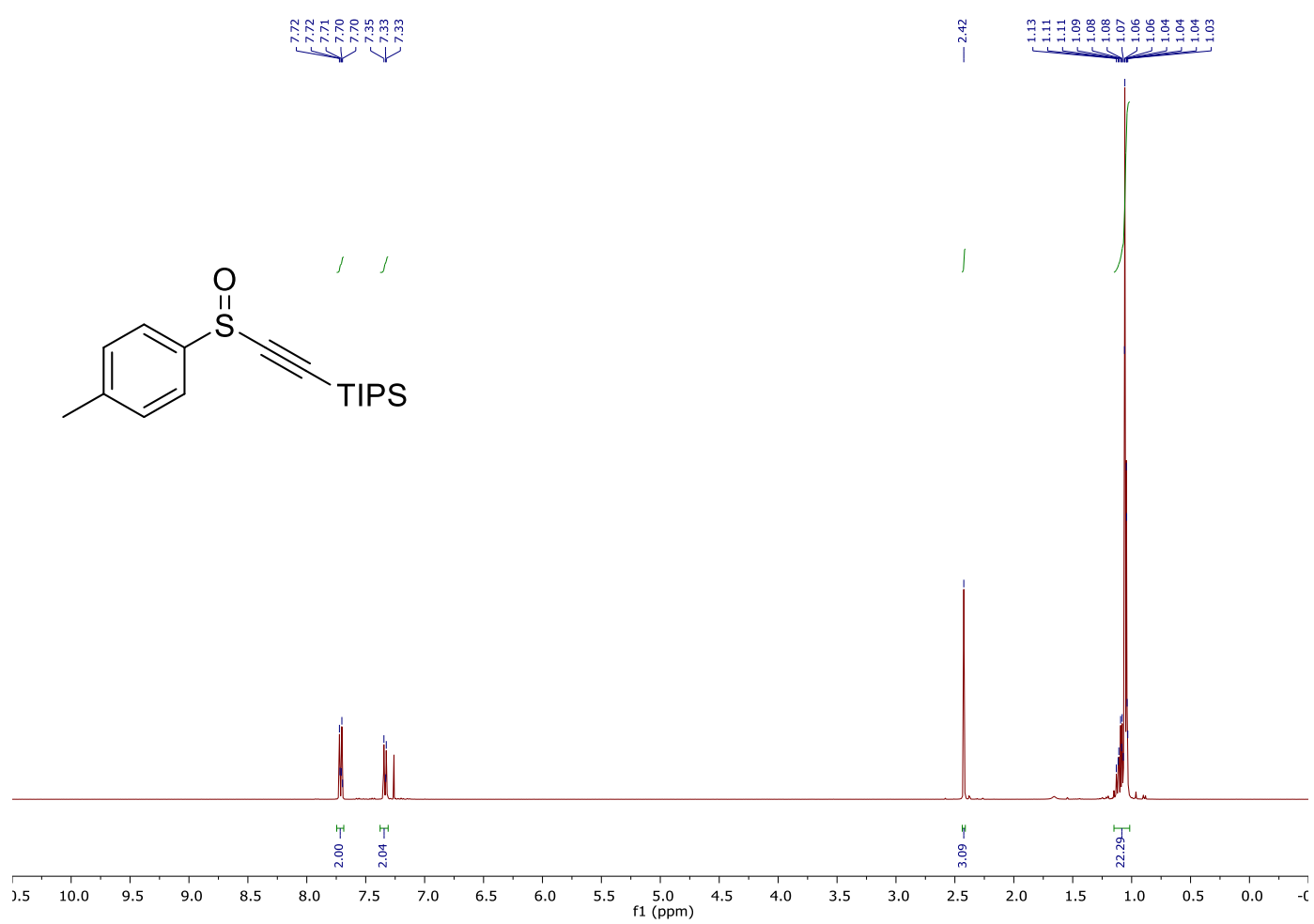

${ }^{13} \mathrm{C}$ NMR (101 MHz, Chloroform- $d$ )

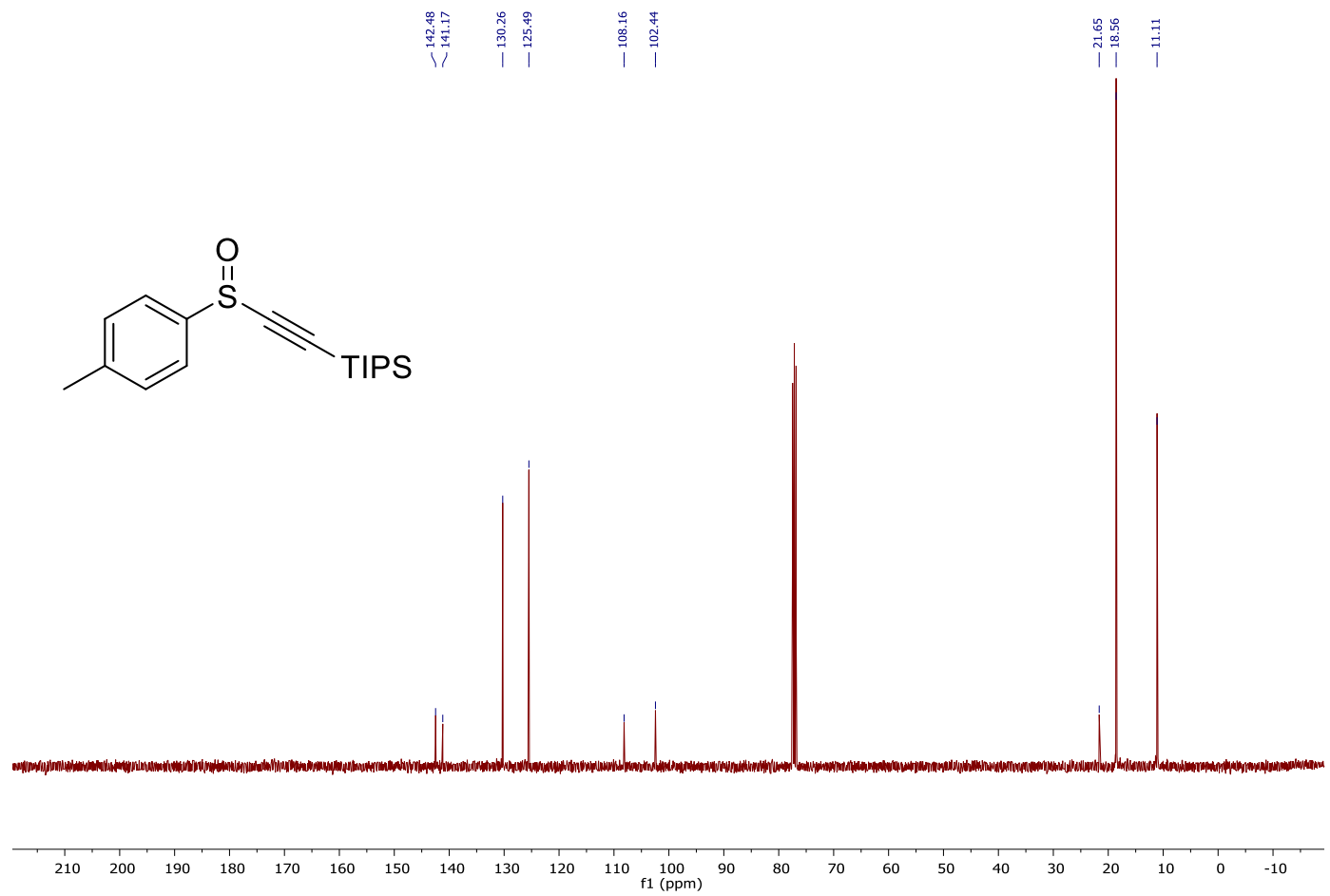


Triisopropyl((phenylsulfinyl)ethynyl)silane (10b)

${ }^{1} \mathrm{H}$ NMR (400 MHz, Chloroform- $d$ )

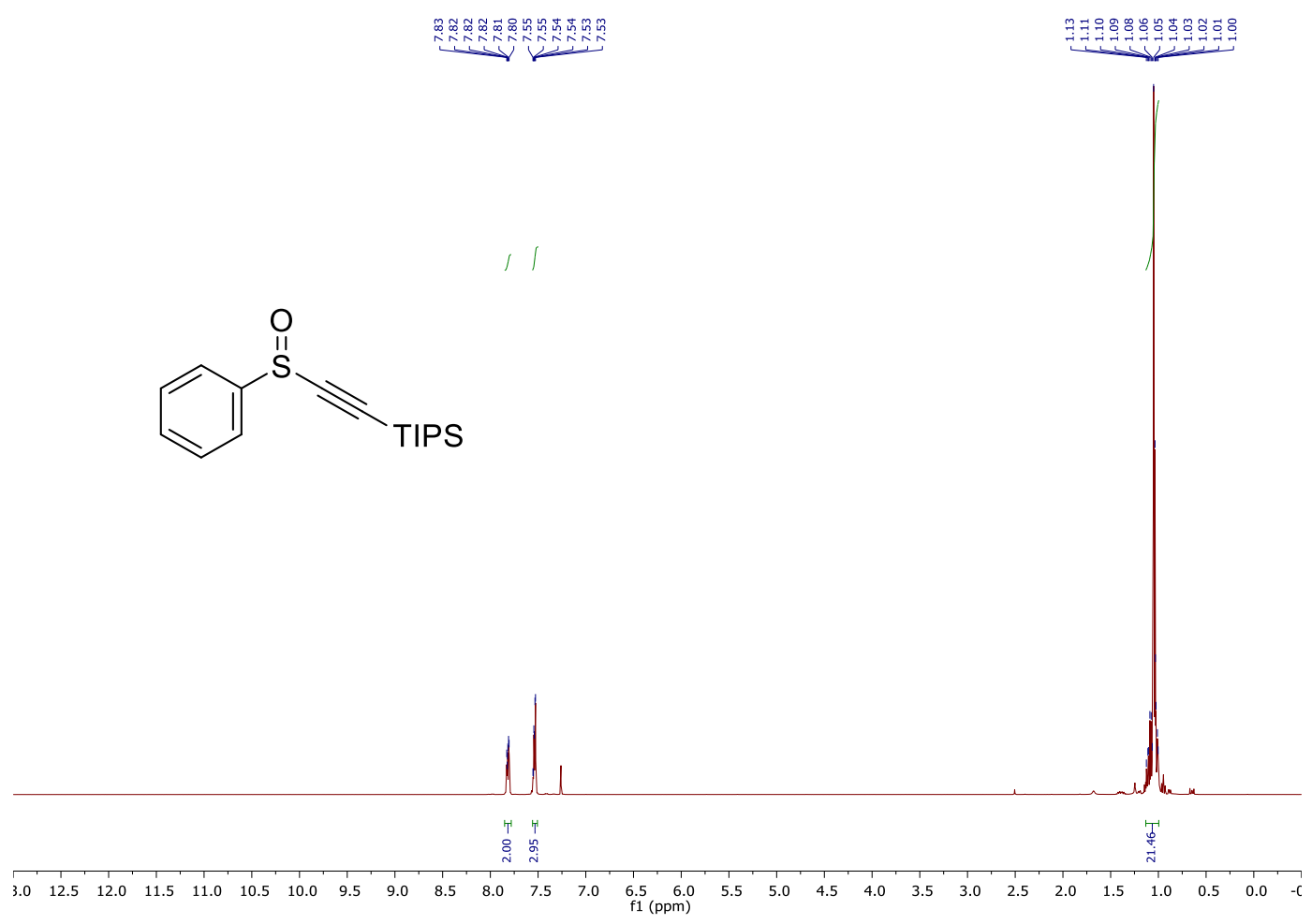

${ }^{13} \mathrm{C}$ NMR (101 MHz, Chloroform- $d$ )

(1)

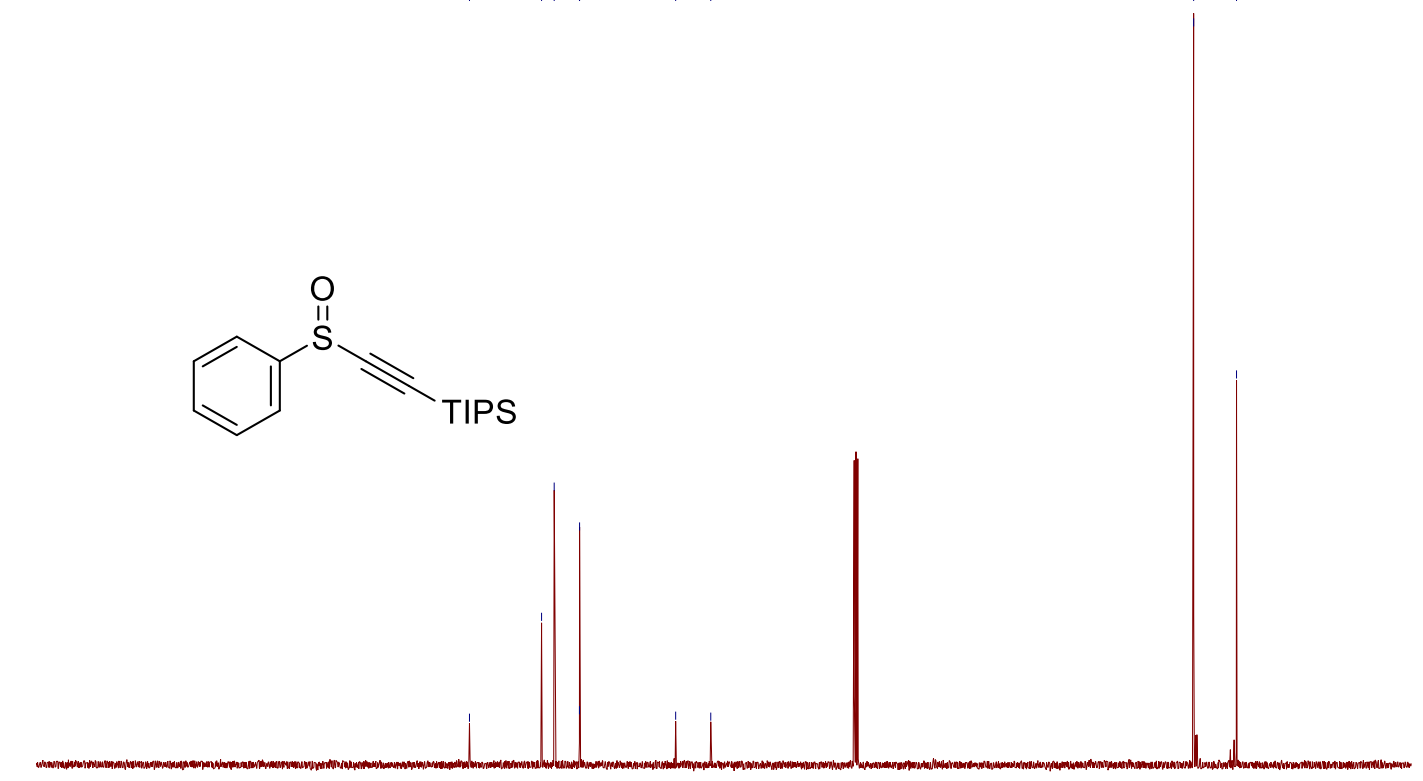

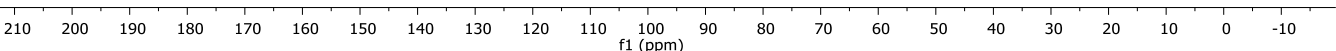




\section{Triisopropyl((4-methoxyphenylsulfinyl)ethynyl)silane (10c)}

${ }^{1} \mathrm{H}$ NMR (400 MHz, Chloroform- $d$ )

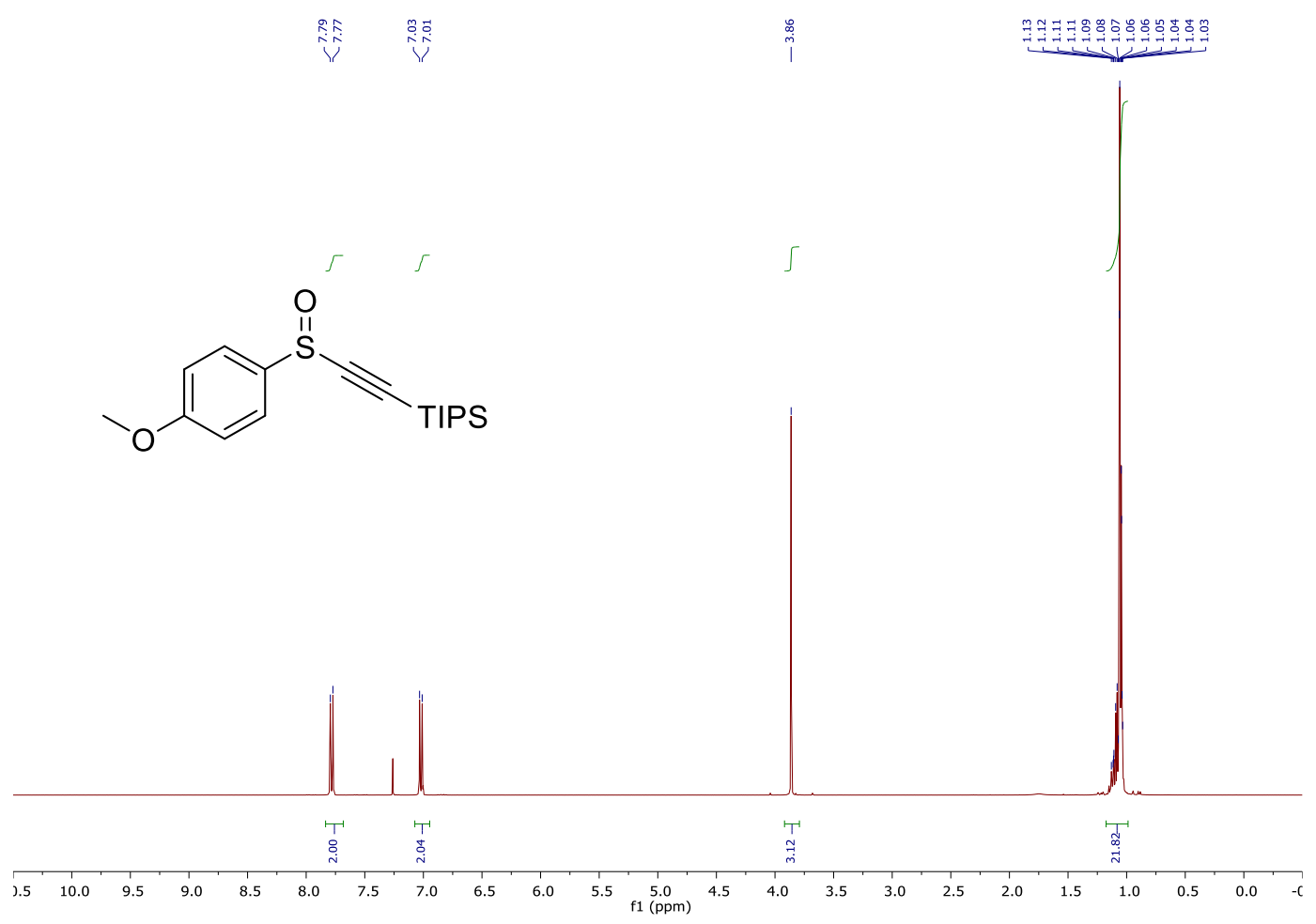

${ }^{13} \mathrm{C}$ NMR (101 MHz, Chloroform- $d$ )
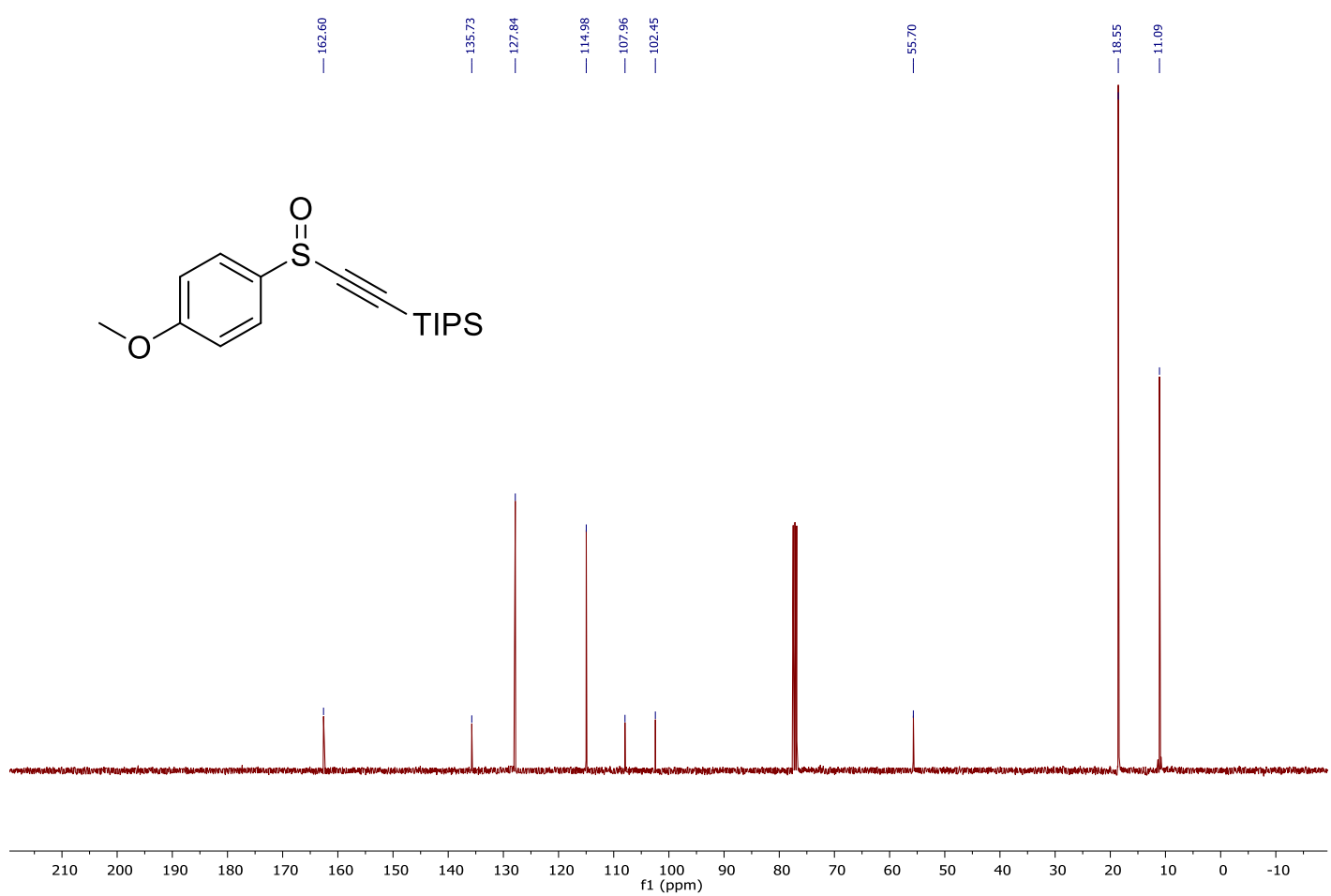
Triisopropyl((4-bromophenylsulfinyl)ethynyl)silane (10d)

${ }^{1} \mathrm{H}$ NMR (400 MHz, Chloroform- $d$ )

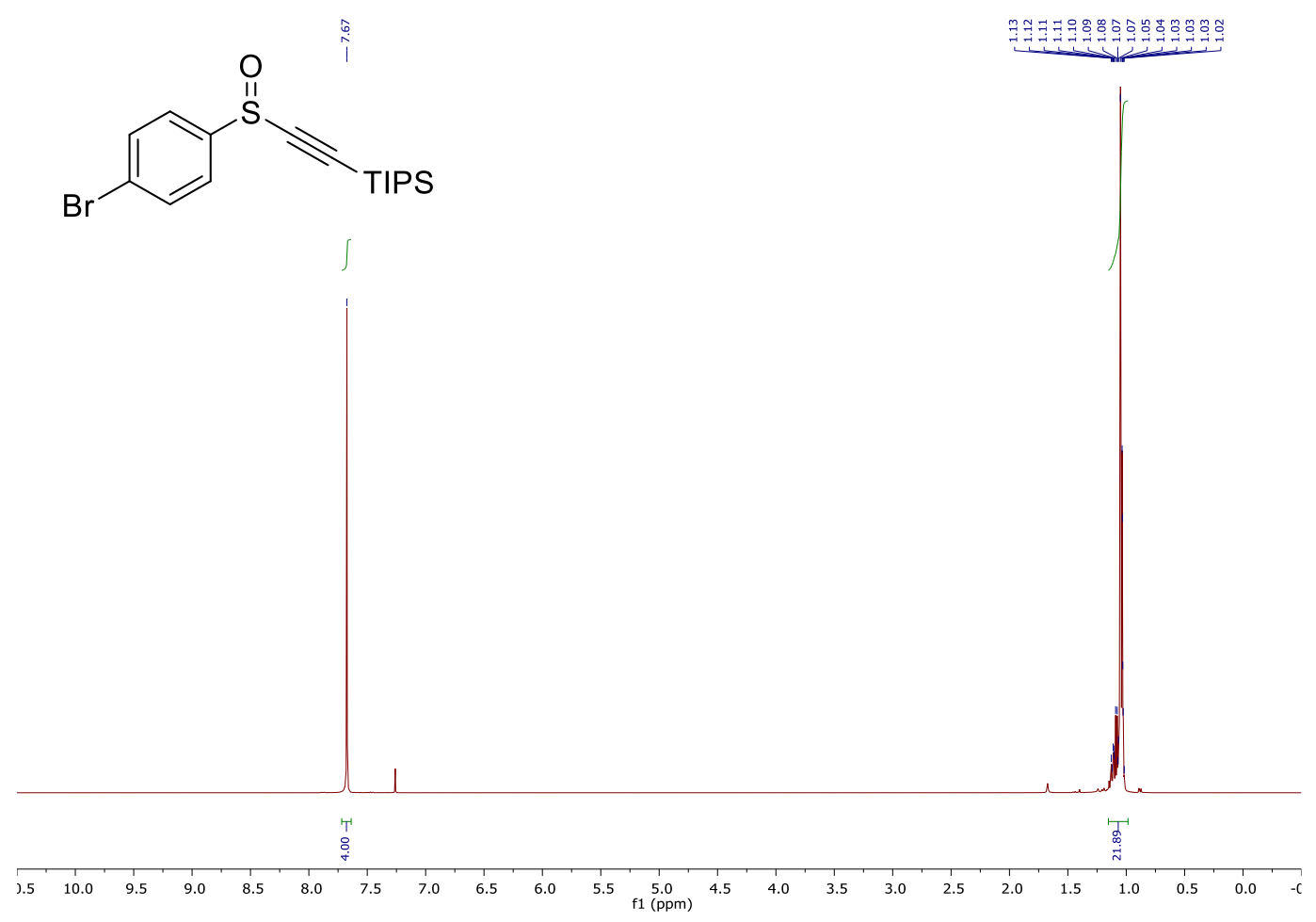

${ }^{13} \mathrm{C}$ NMR (101 MHz, Chloroform- $d$ )

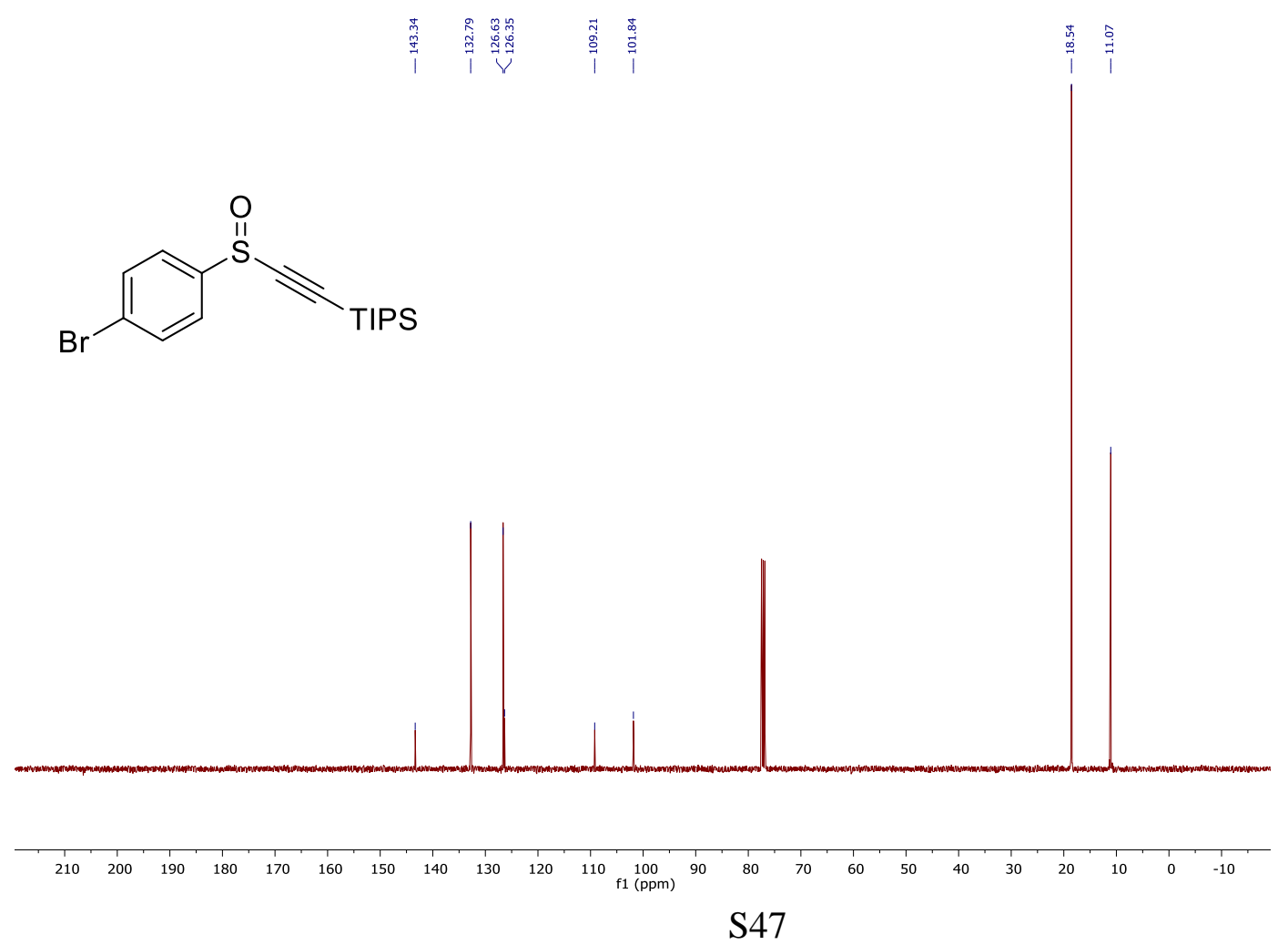




\section{Triisopropyl((4-fluorophenylsulfinyl)ethynyl)silane (10e)}

${ }^{1} \mathrm{H}$ NMR (400 MHz, Chloroform- $d$ )

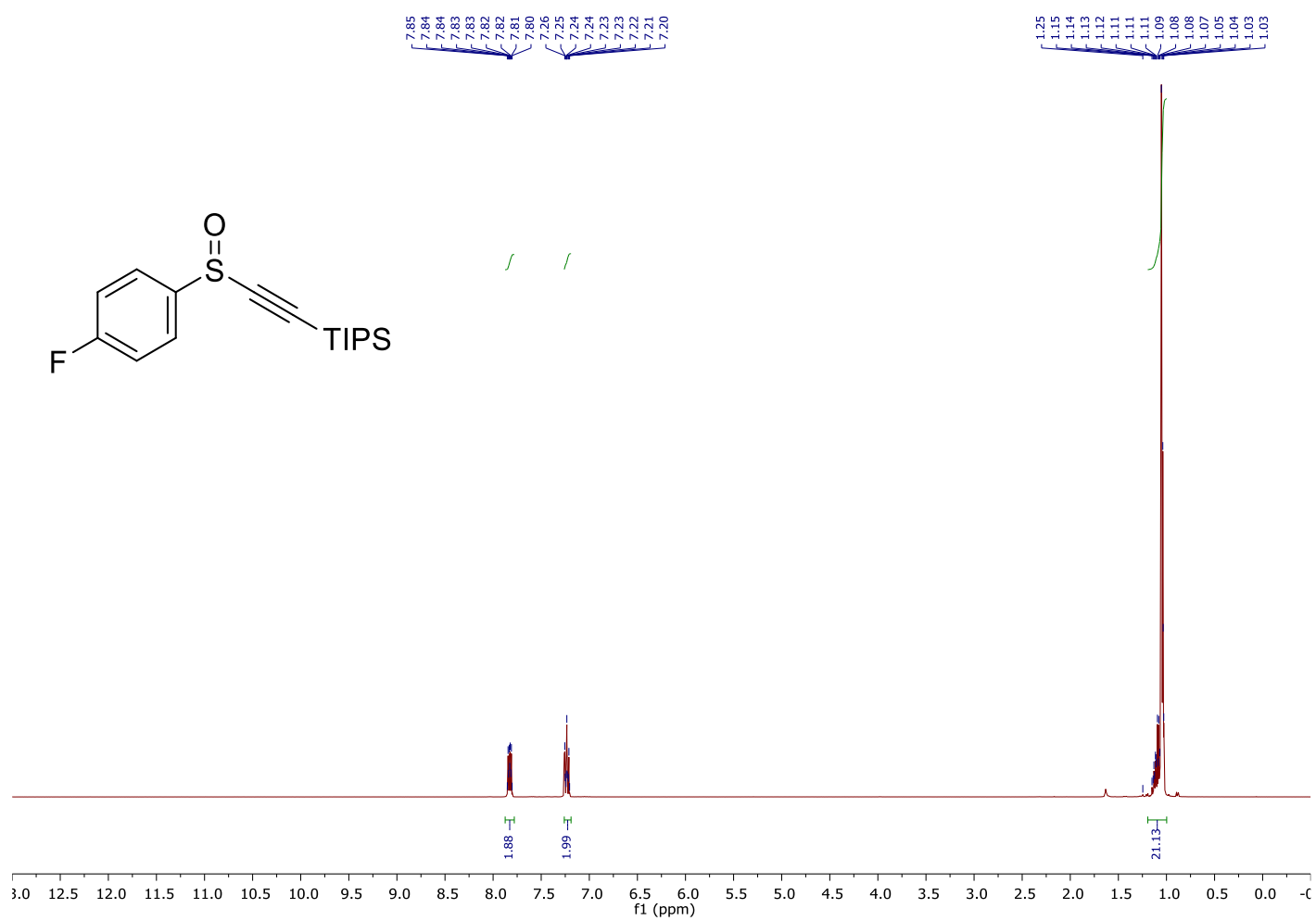

${ }^{13} \mathrm{C}$ NMR (101 MHz, Chloroform- $d$ )
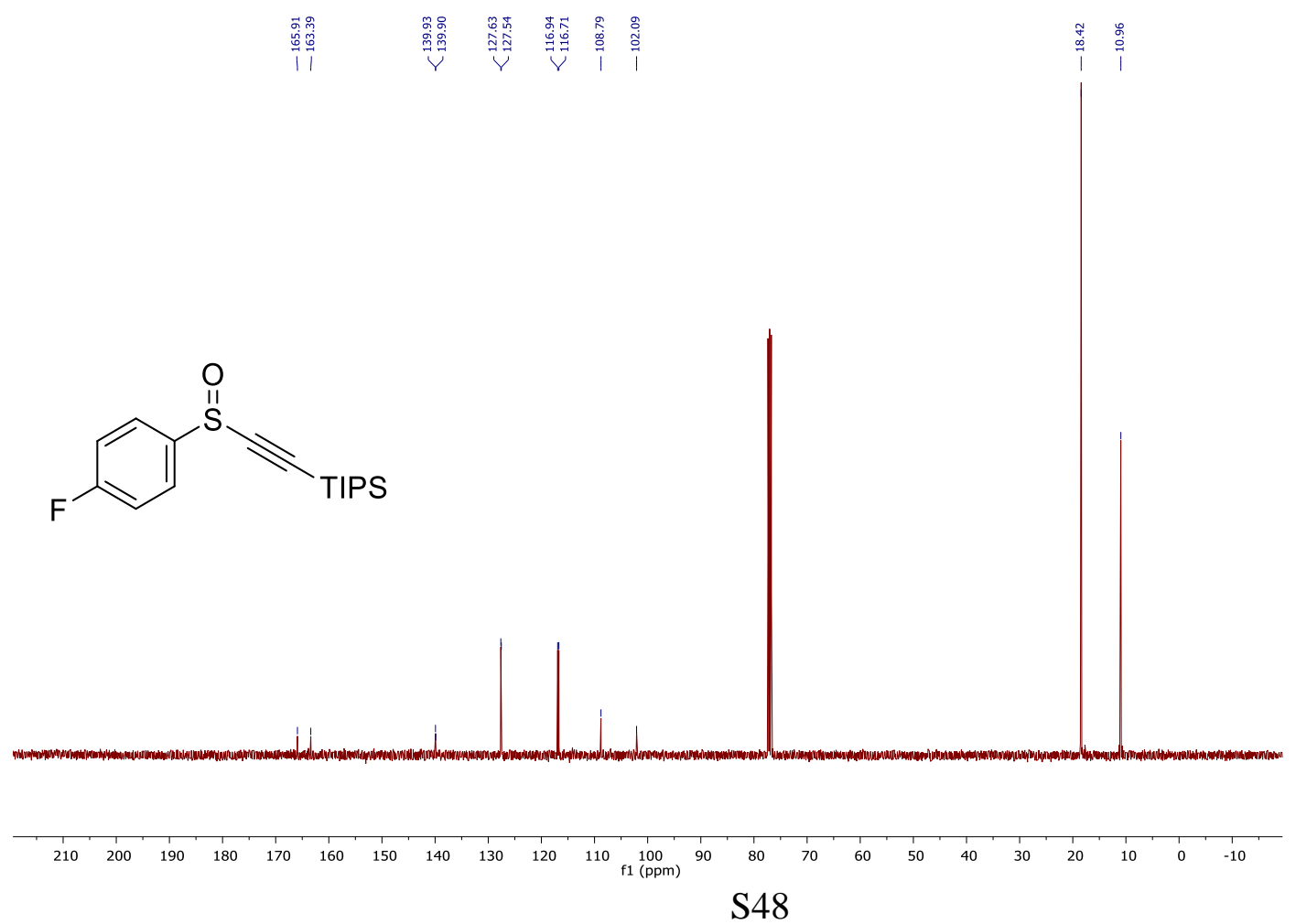
${ }^{19}$ F NMR (376 MHz, Chloroform-d)

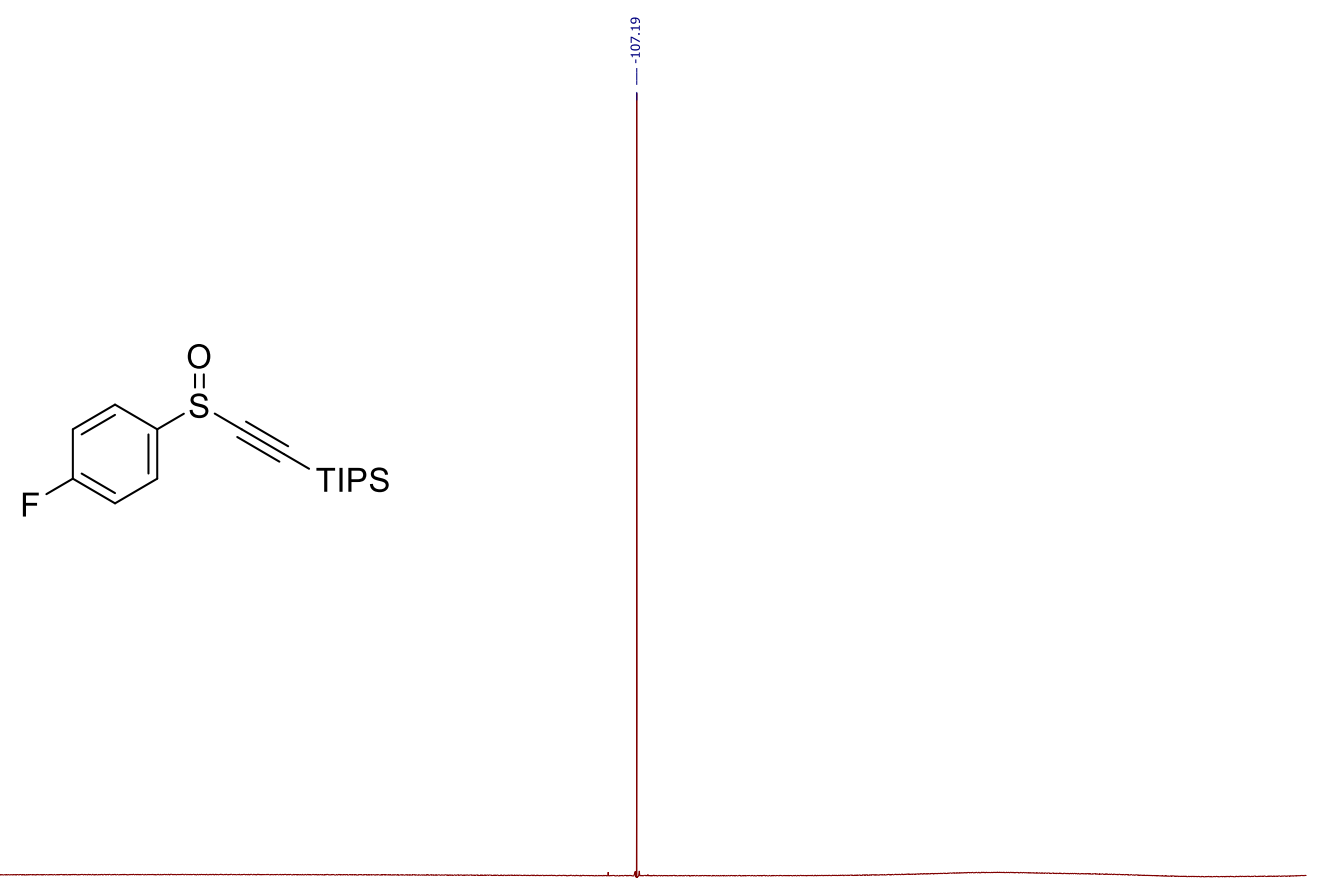

$\begin{array}{lllllllllllllllllllllll}10 & 0 & -10 & -20 & -30 & -40 & -50 & -60 & -70 & -80 & -90 & -100 & -110 & -120 & -130 & -140 & -150 & -160 & -170 & -180 & -190 & -200 & -210\end{array}$ 


\section{Triisopropyl((4-trifluoromethylphenylsulfinyl)ethynyl)silane (10f)}

${ }^{1} \mathrm{H}$ NMR (400 MHz, Chloroform- $d$ )

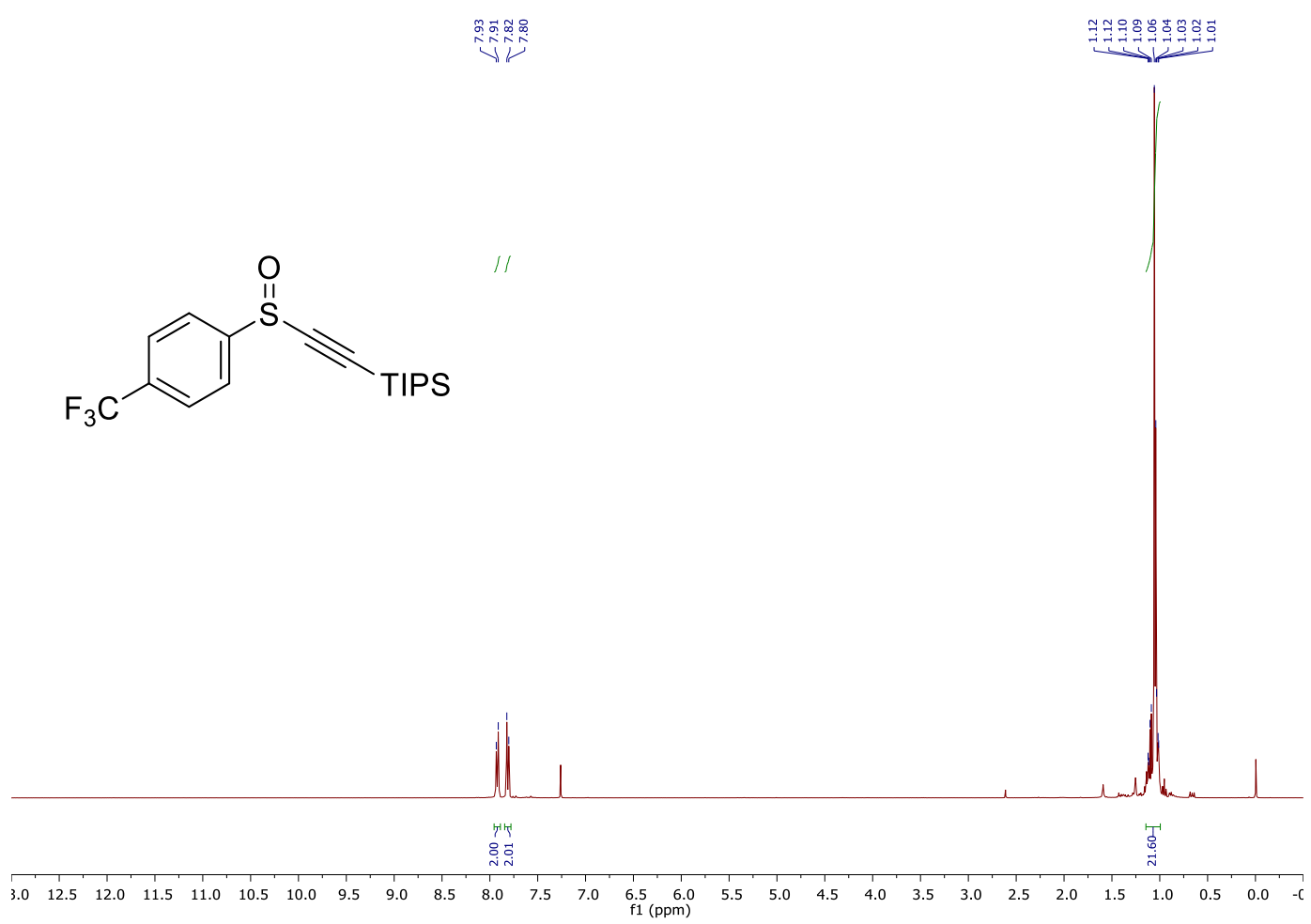

${ }^{13} \mathrm{C}$ NMR (101 MHz, Chloroform- $d$ )

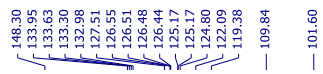

$\overbrace{\text { TIPS }}^{\longrightarrow}$
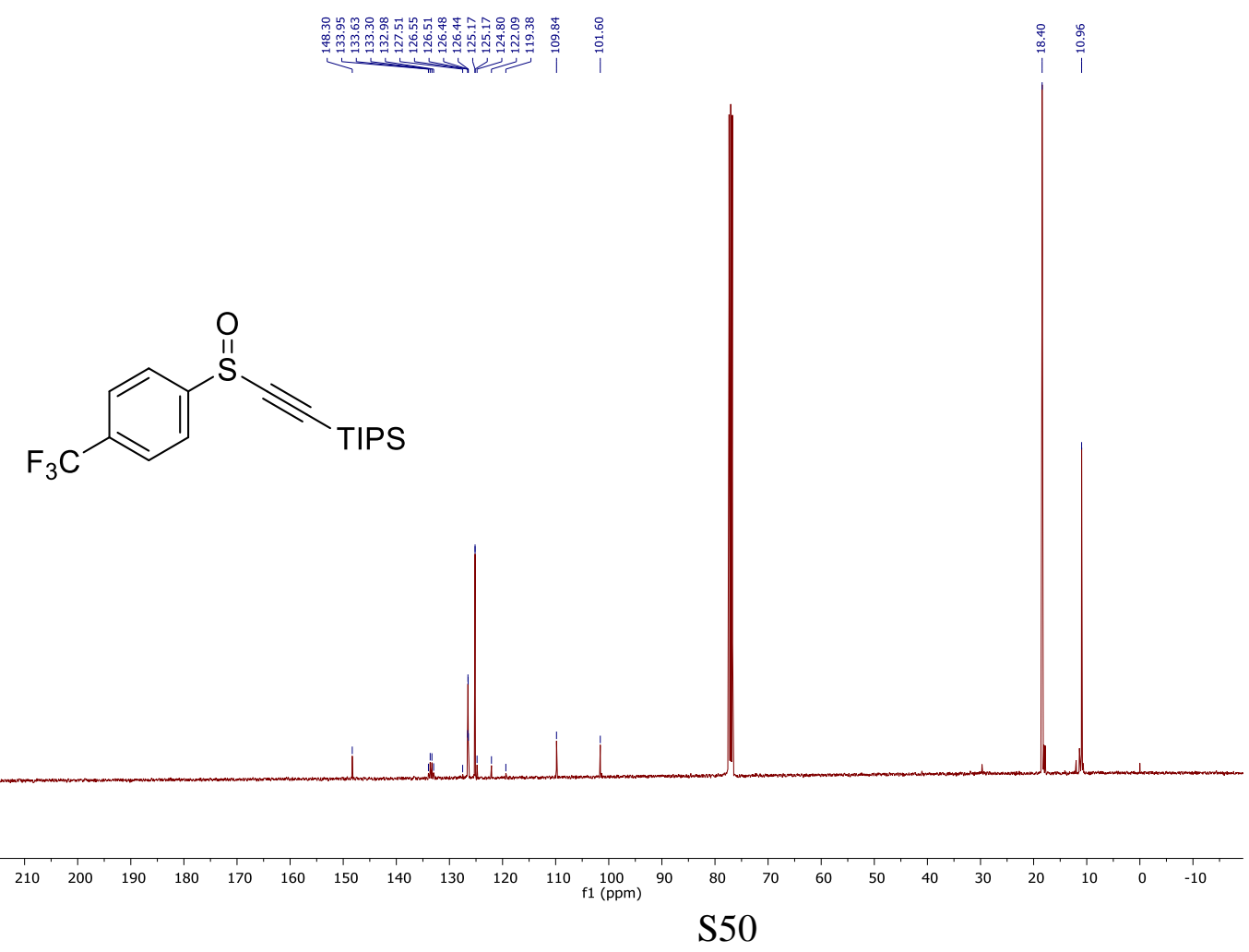
${ }^{19}$ F NMR (376 MHz, Chloroform-d)

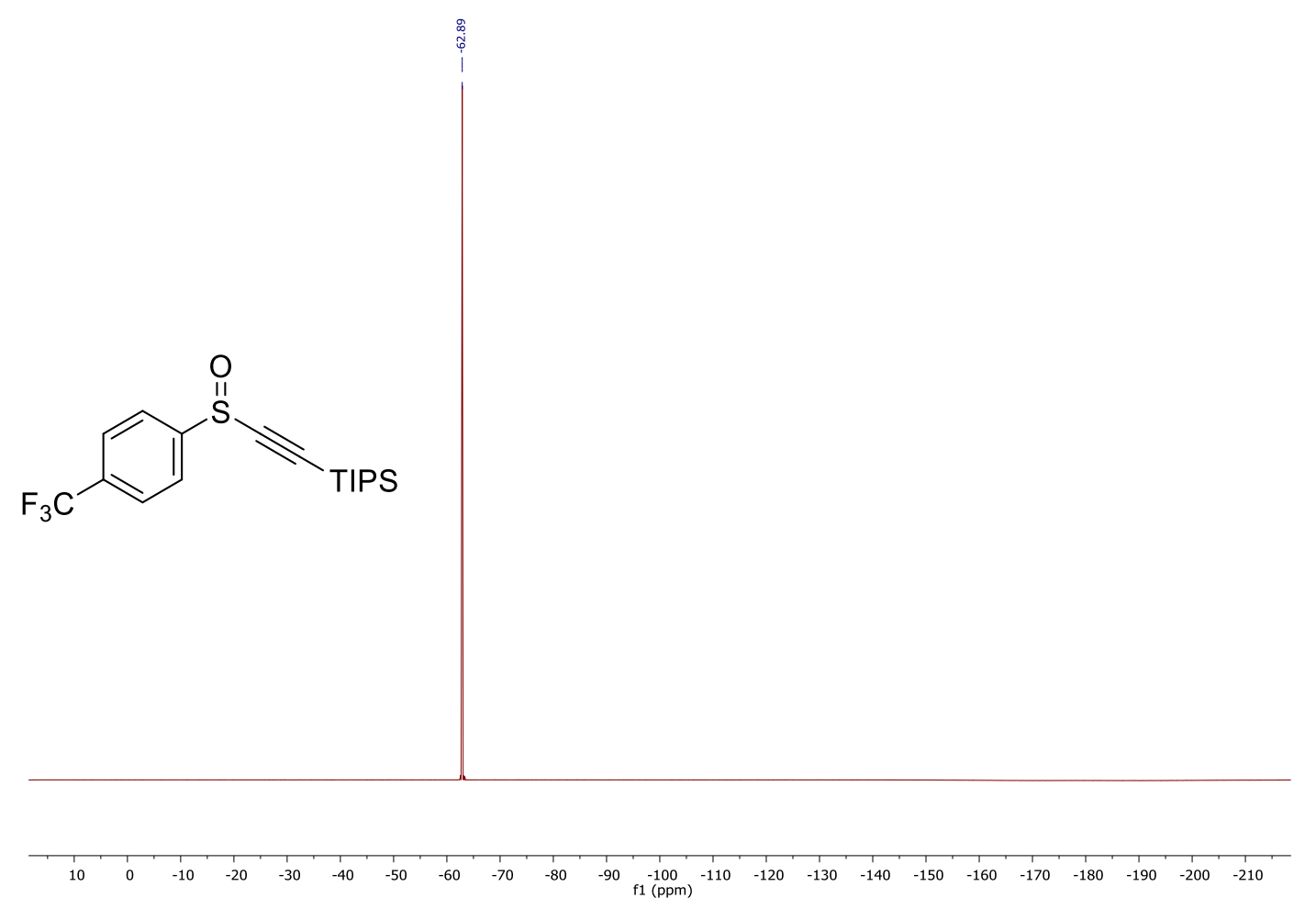




\section{Triisopropyl((4-nitrophenylsulfinyl)ethynyl)silane (10g)}

${ }^{1} \mathrm{H}$ NMR (400 MHz, Chloroform- $d$ )

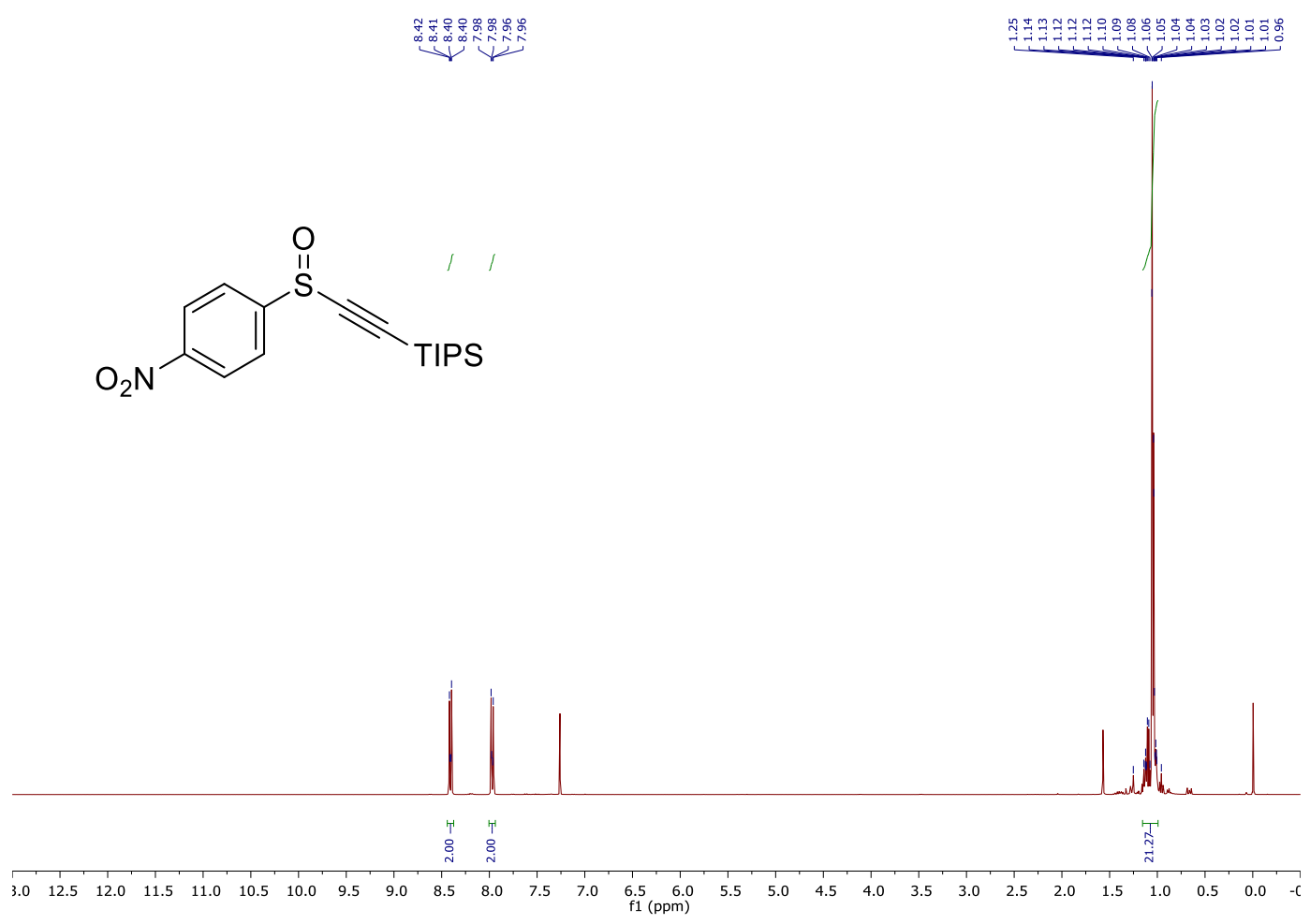

${ }^{13} \mathrm{C}$ NMR (101 MHz, Chloroform- $d$ )

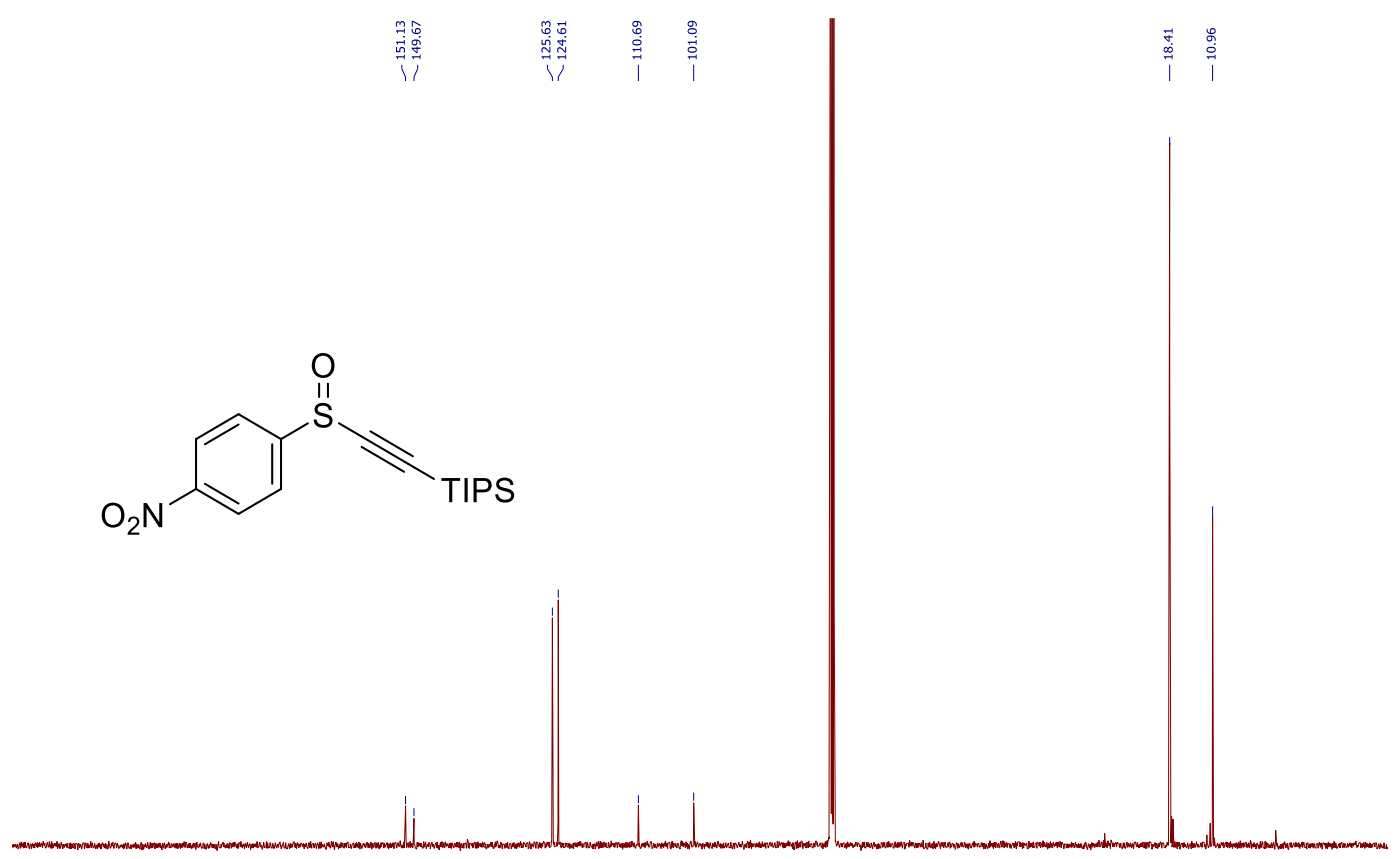

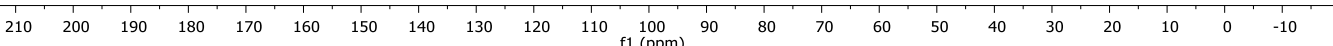




\section{Triisopropyl((2-bromophenylsulfinyl)ethynyl)silane (10h)}

${ }^{1} \mathrm{H}$ NMR (400 MHz, Chloroform- $d$ )

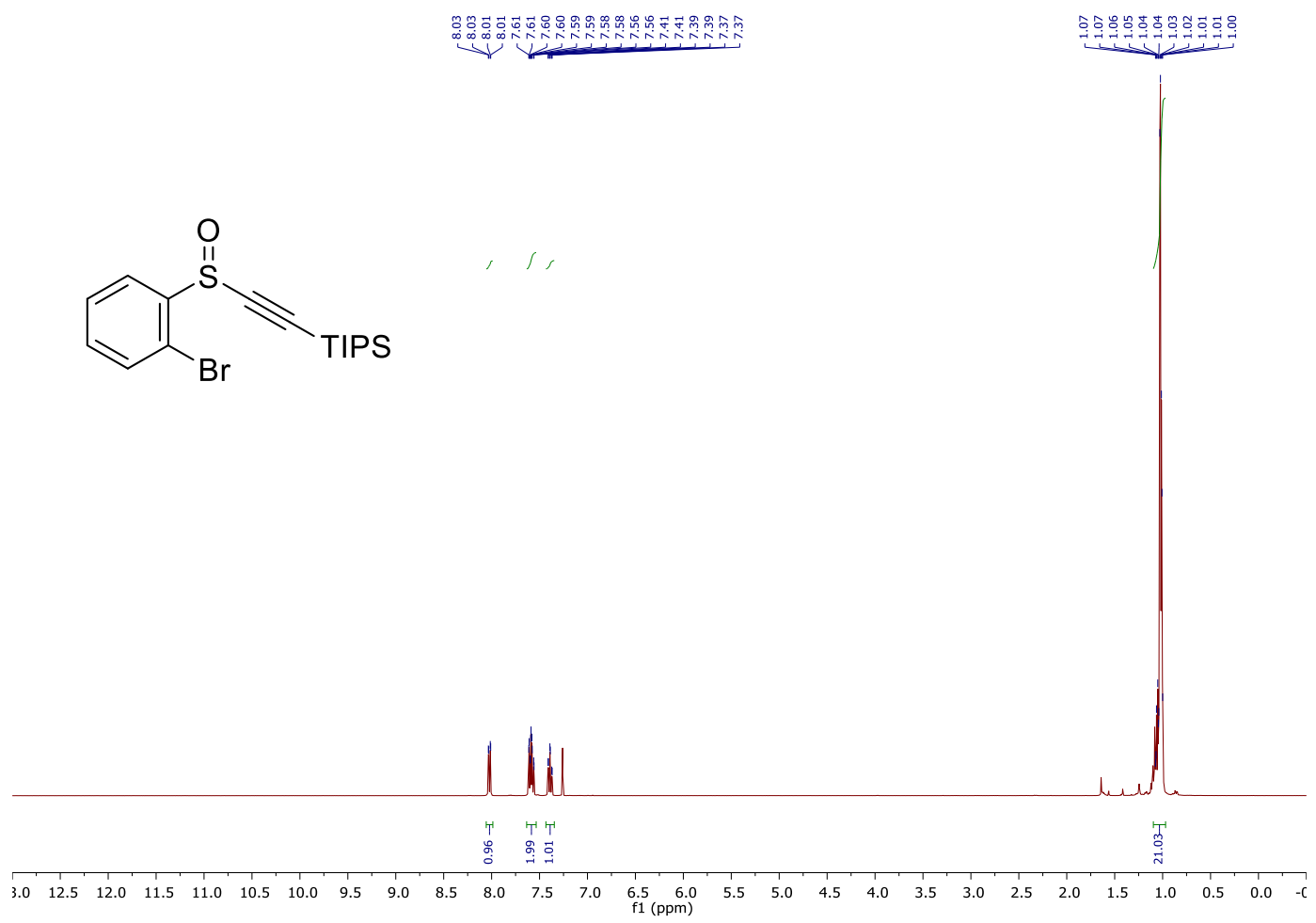

${ }^{13} \mathrm{C}$ NMR (101 MHz, Chloroform- $d$ )

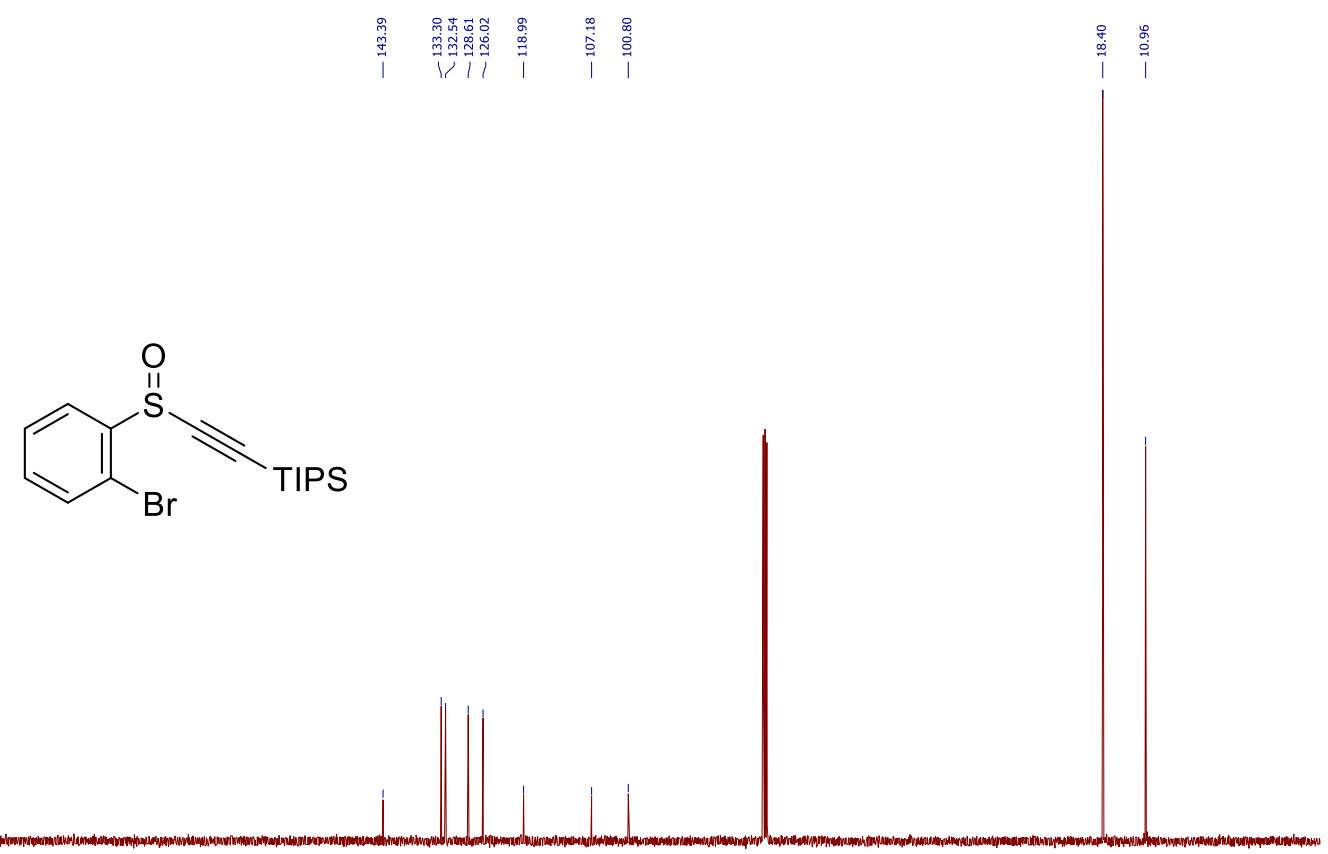

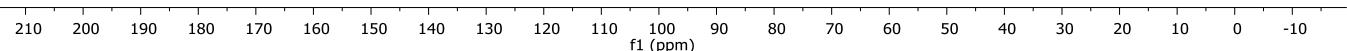




\section{Triisopropyl((3-methylphenylsulfinyl)ethynyl)silane (10i)}

${ }^{1} \mathrm{H}$ NMR (400 MHz, Chloroform- $d$ )

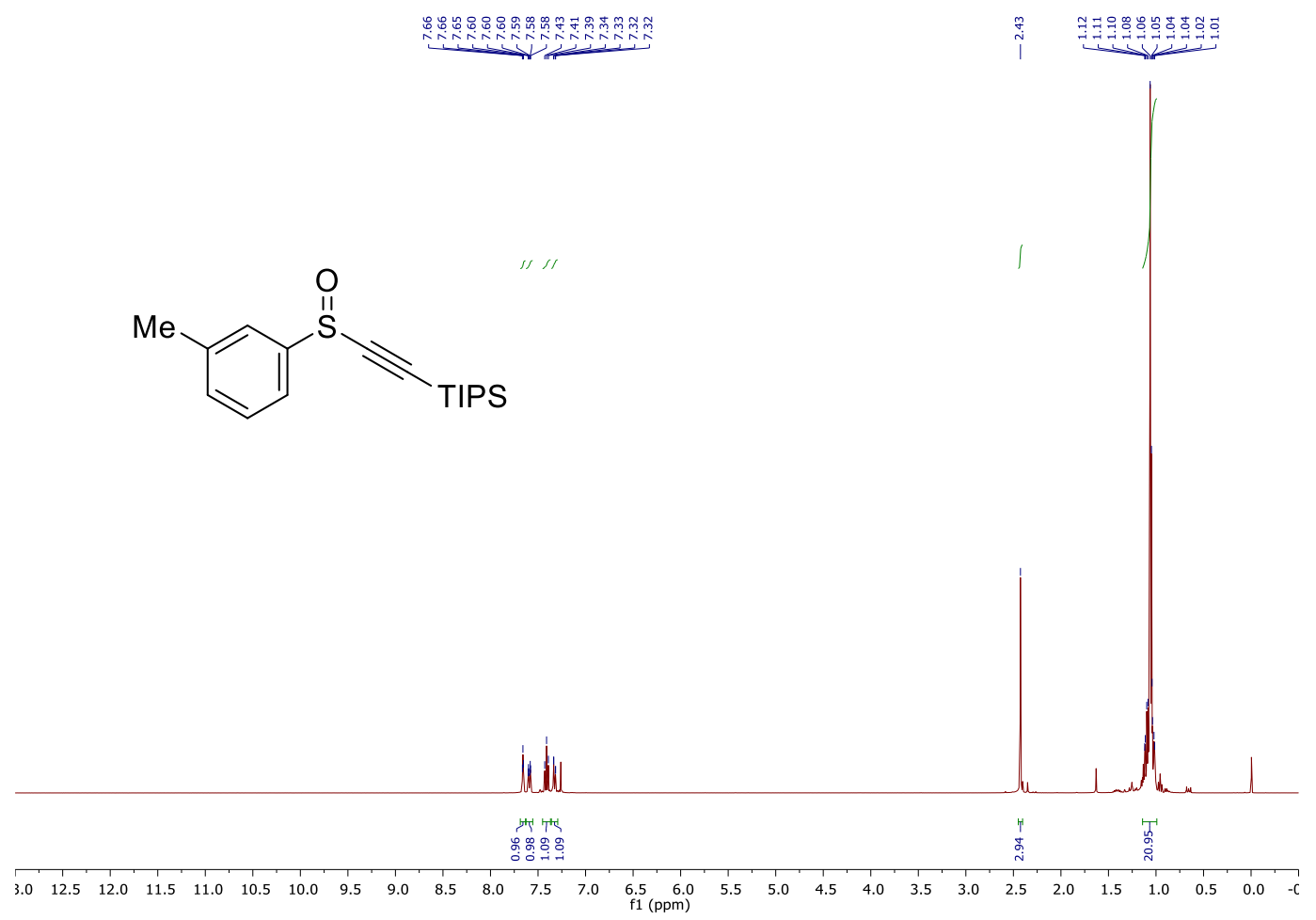

${ }^{13} \mathrm{C}$ NMR (101 MHz, Chloroform- $d$ )
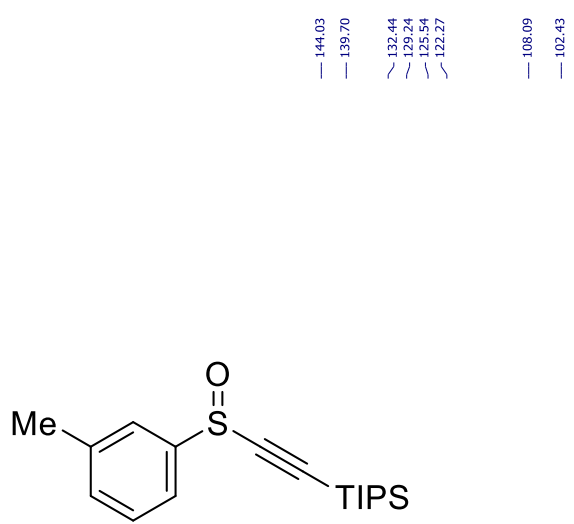

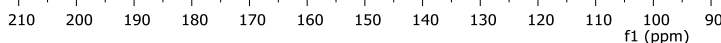


Triisopropyl((3-bromophenylsulfinyl)ethynyl)silane (10j)

${ }^{1} \mathrm{H}$ NMR (400 MHz, Chloroform- $d$ )

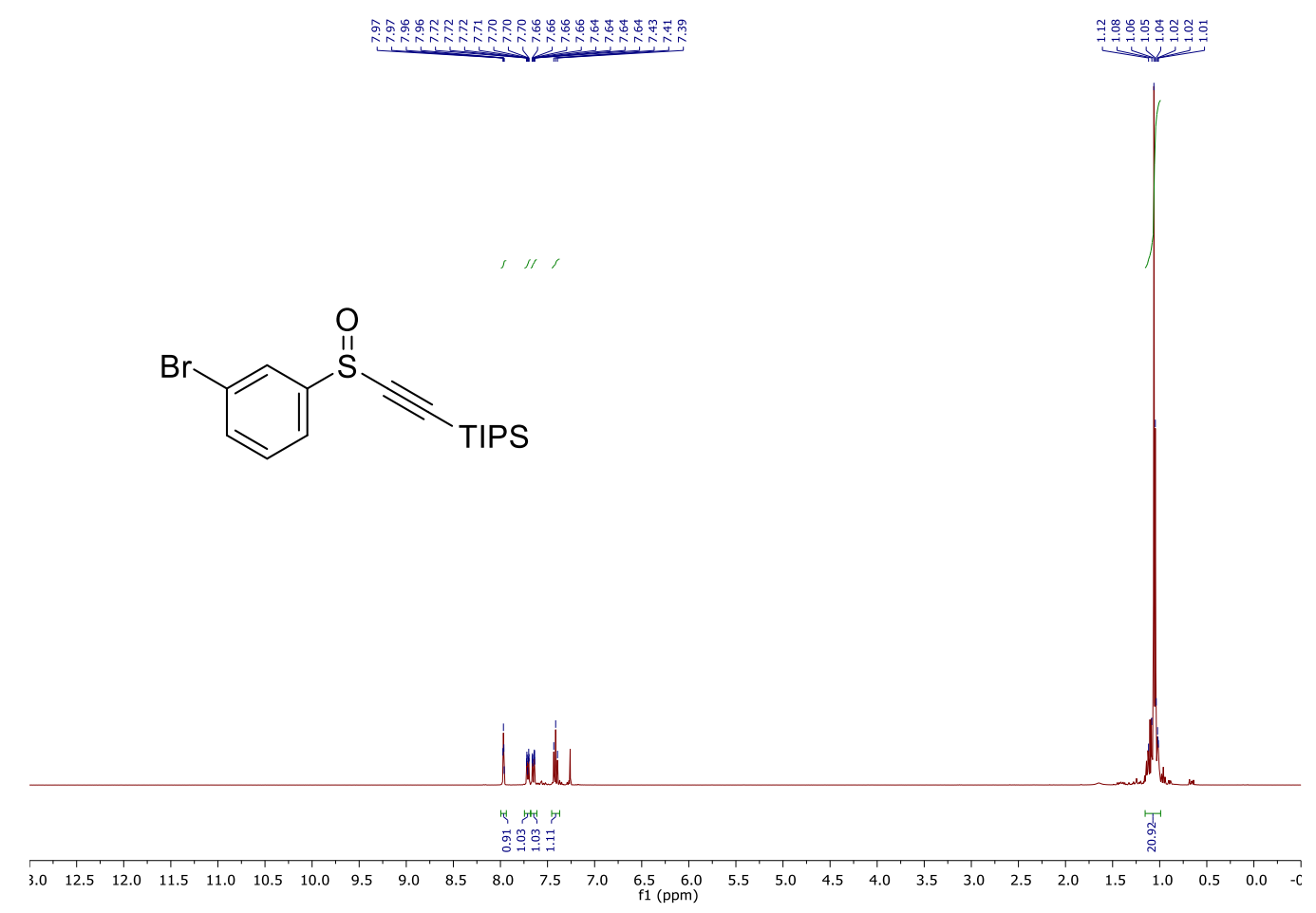

${ }^{13} \mathrm{C}$ NMR (101 MHz, Chloroform- $d$ )

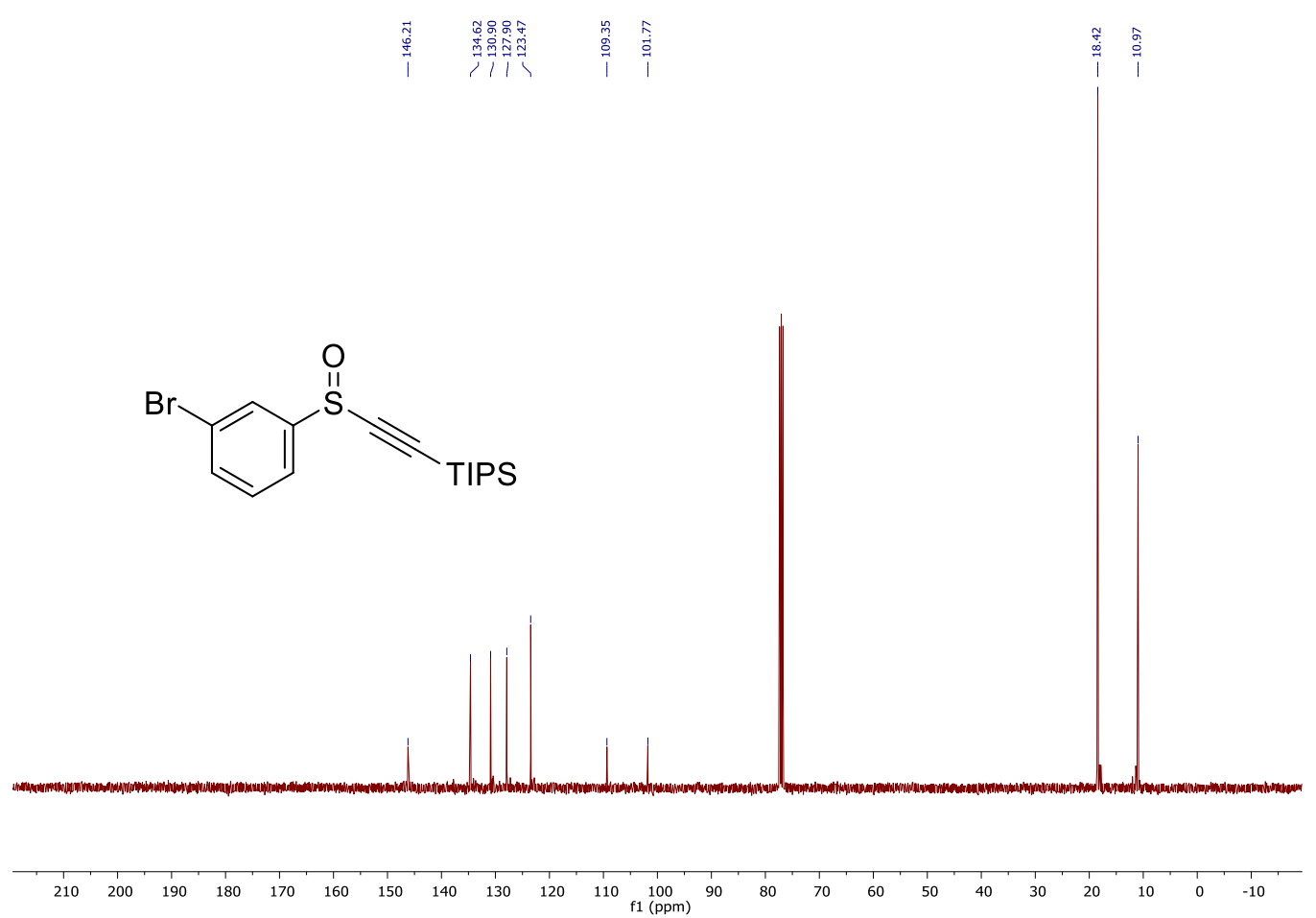




\section{Triisopropyl((pyridin-2-ylsulfinyl)ethynyl)silane (10k)}

${ }^{1} \mathrm{H}$ NMR (400 MHz, Chloroform- $d$ )

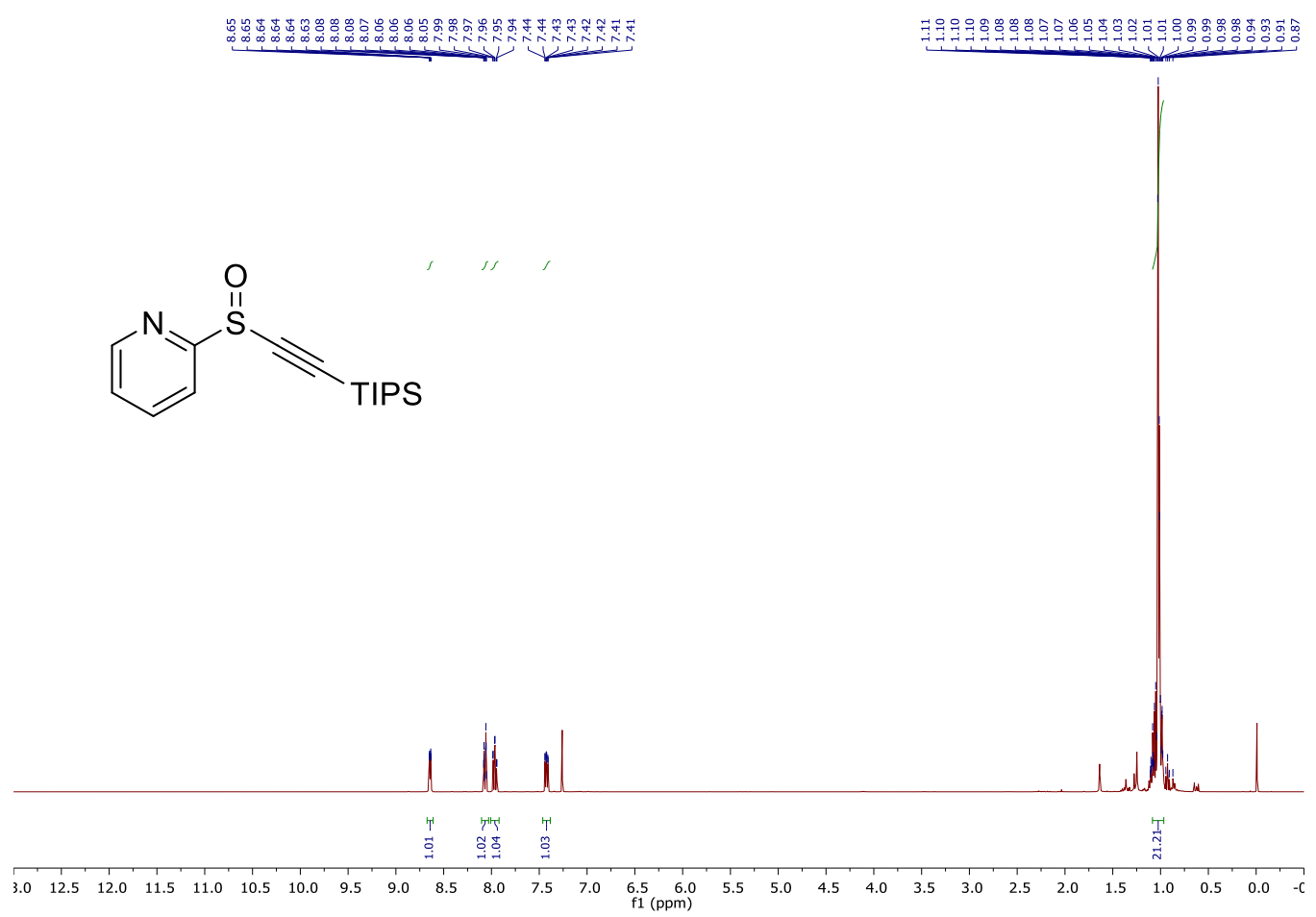

${ }^{13} \mathrm{C}$ NMR (101 MHz, Chloroform- $d$ )

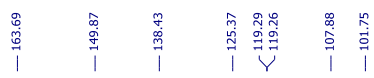<smiles>O=S(C#C[In])c1ccccn1</smiles>

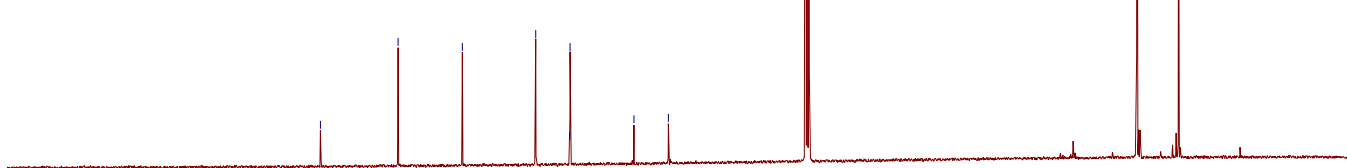

$\begin{array}{lllllllllll}210 & 200 & 190 & 180 & 170 & 160 & 150 & 140 & 130 & 120 & 110 \underset{\mathrm{f} 1(\mathrm{ppm})}{100} 90\end{array}$ 


\section{Triisopropyl((thiophen-2-ylsulfinyl)ethynyl)silane (10l)}

${ }^{1} \mathrm{H}$ NMR (400 MHz, Chloroform-d)

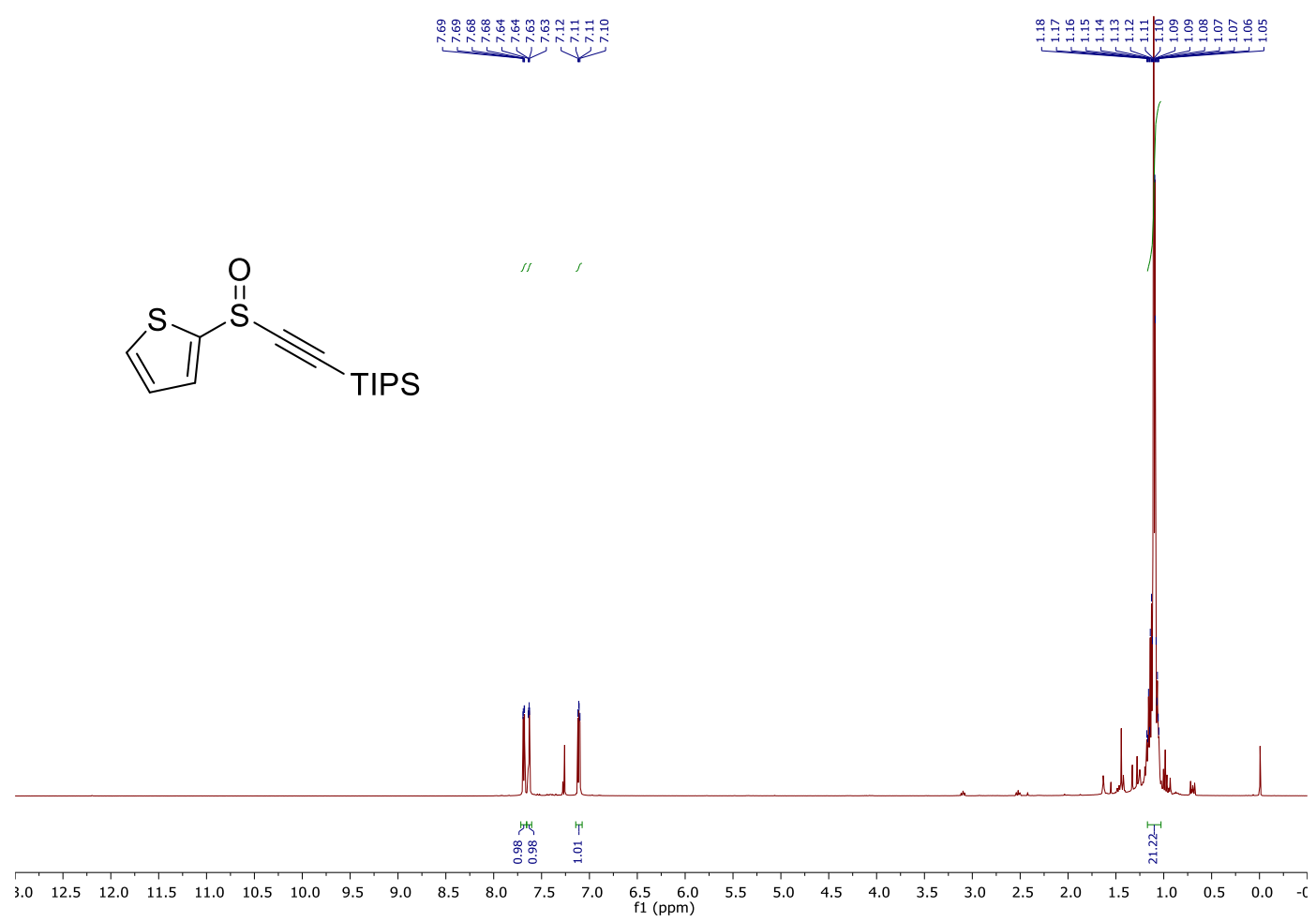

${ }^{13} \mathrm{C}$ NMR (101 MHz, Chloroform-d)

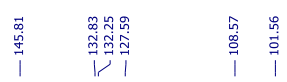
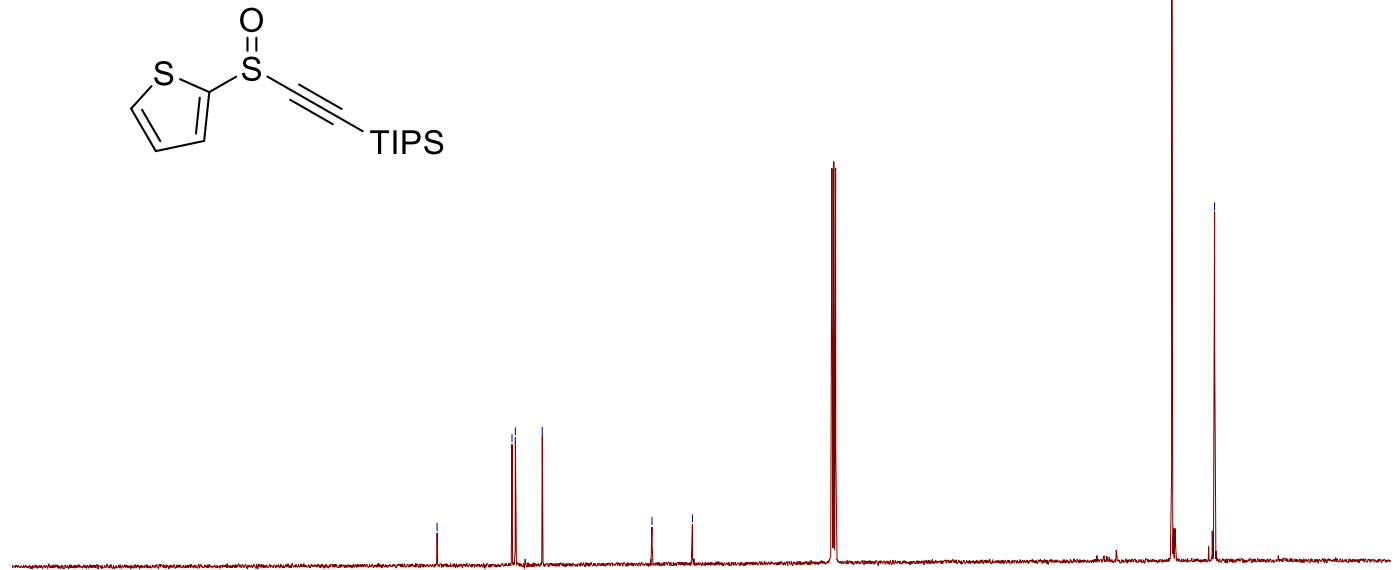

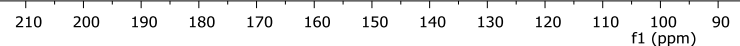


Triisopropyl((2-methylfuran-3-ylsulfinyl)ethynyl)silane (10m)

${ }^{1} \mathrm{H}$ NMR (400 MHz, Chloroform- $d$ )

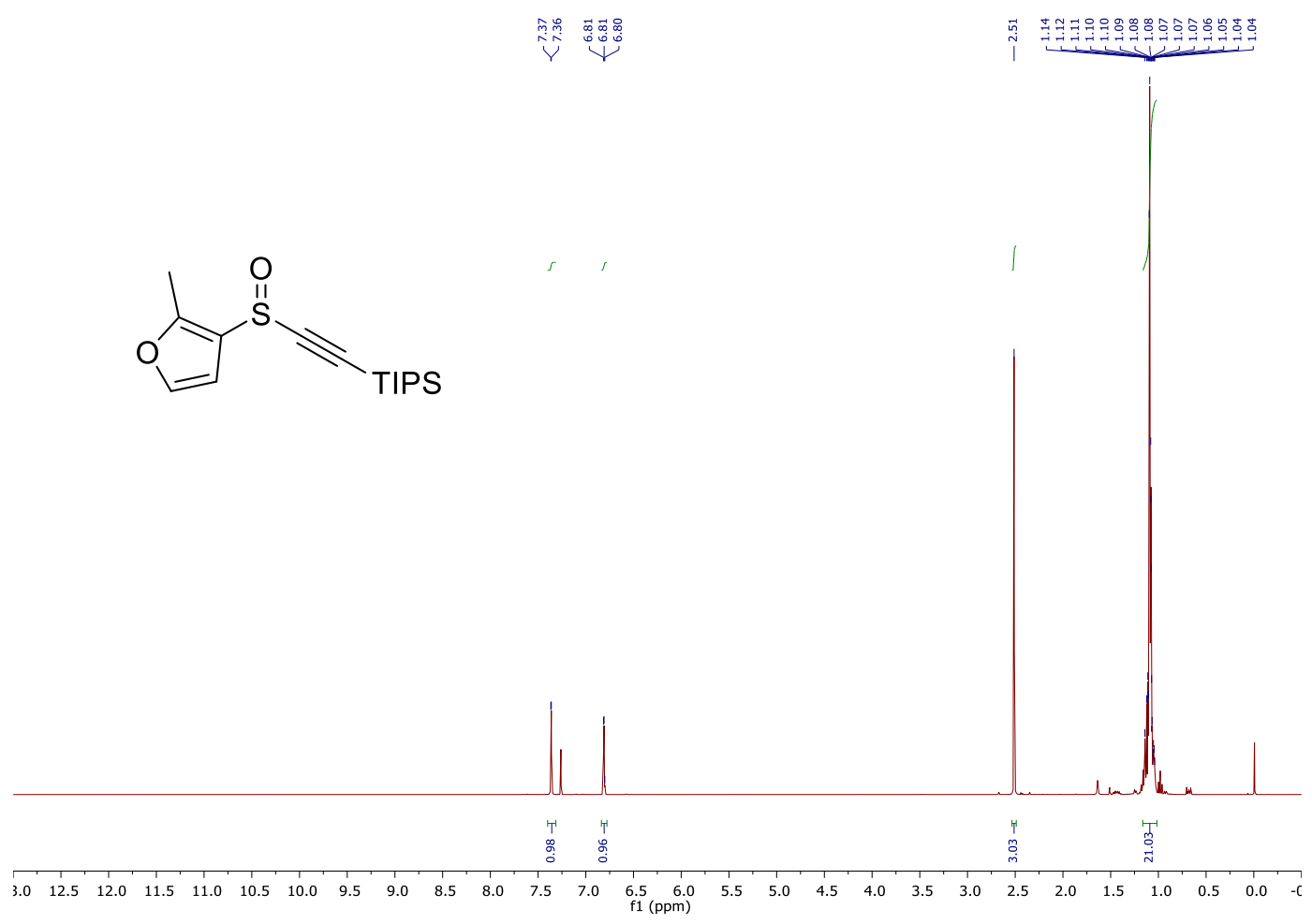

${ }^{13} \mathrm{C}$ NMR (101 MHz, Chloroform- $\left.d\right)$

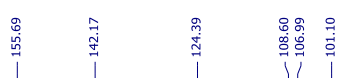<smiles>Cc1occc1S(=O)C#C[In]S</smiles>

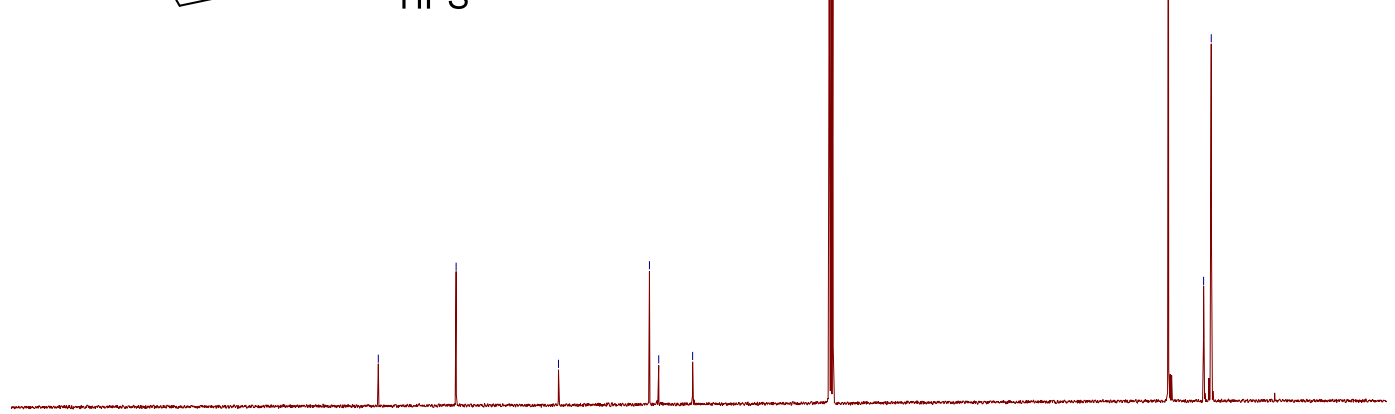

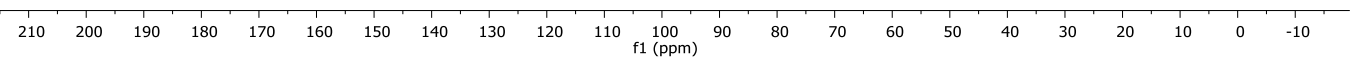
S58 


\section{Triisopropyl((pentylsulfinyl)ethynyl)silane (10n)}

${ }^{1} \mathrm{H}$ NMR (400 MHz, Chloroform-d)

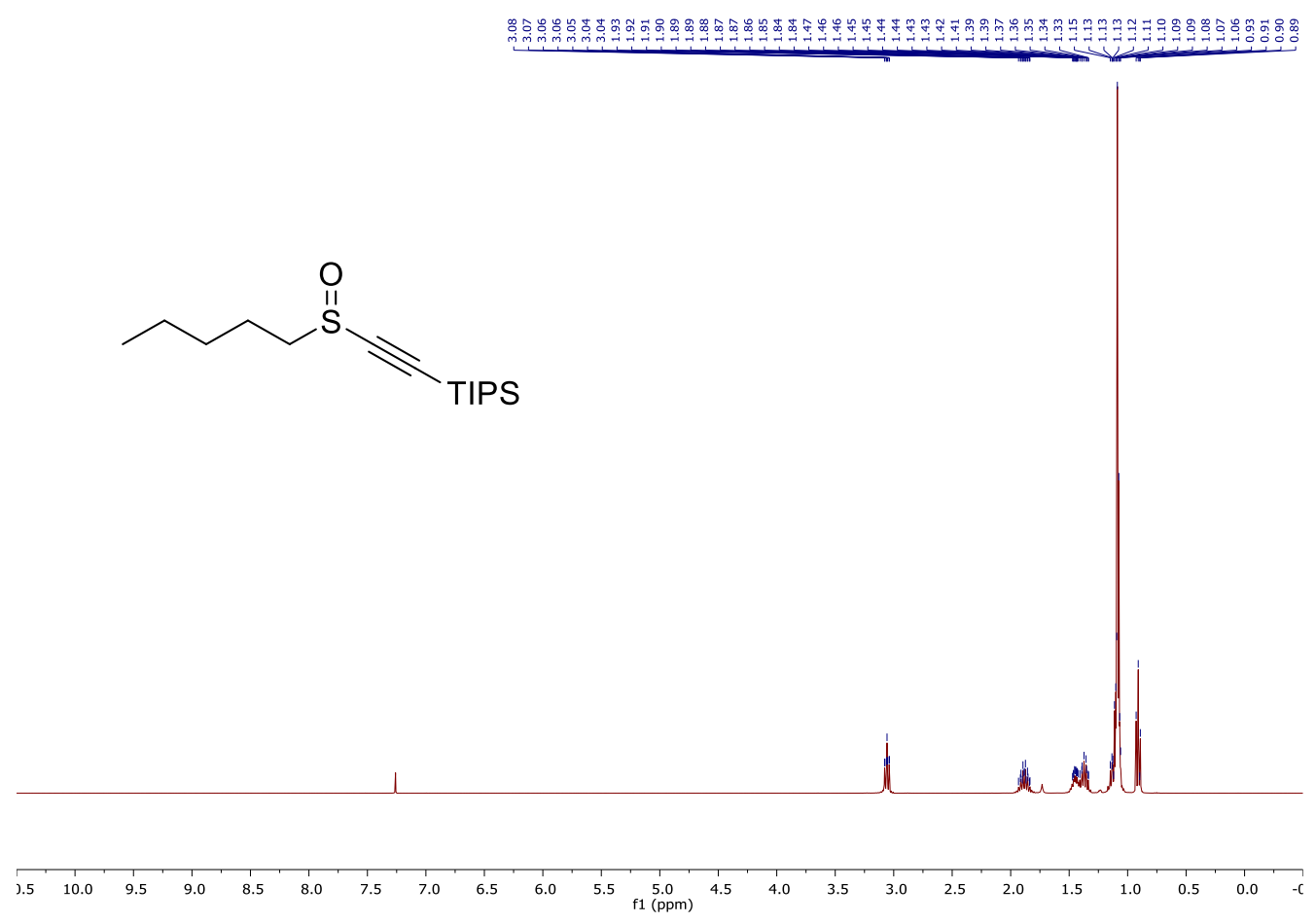

${ }^{13} \mathrm{C}$ NMR (101 MHz, Chloroform-d)

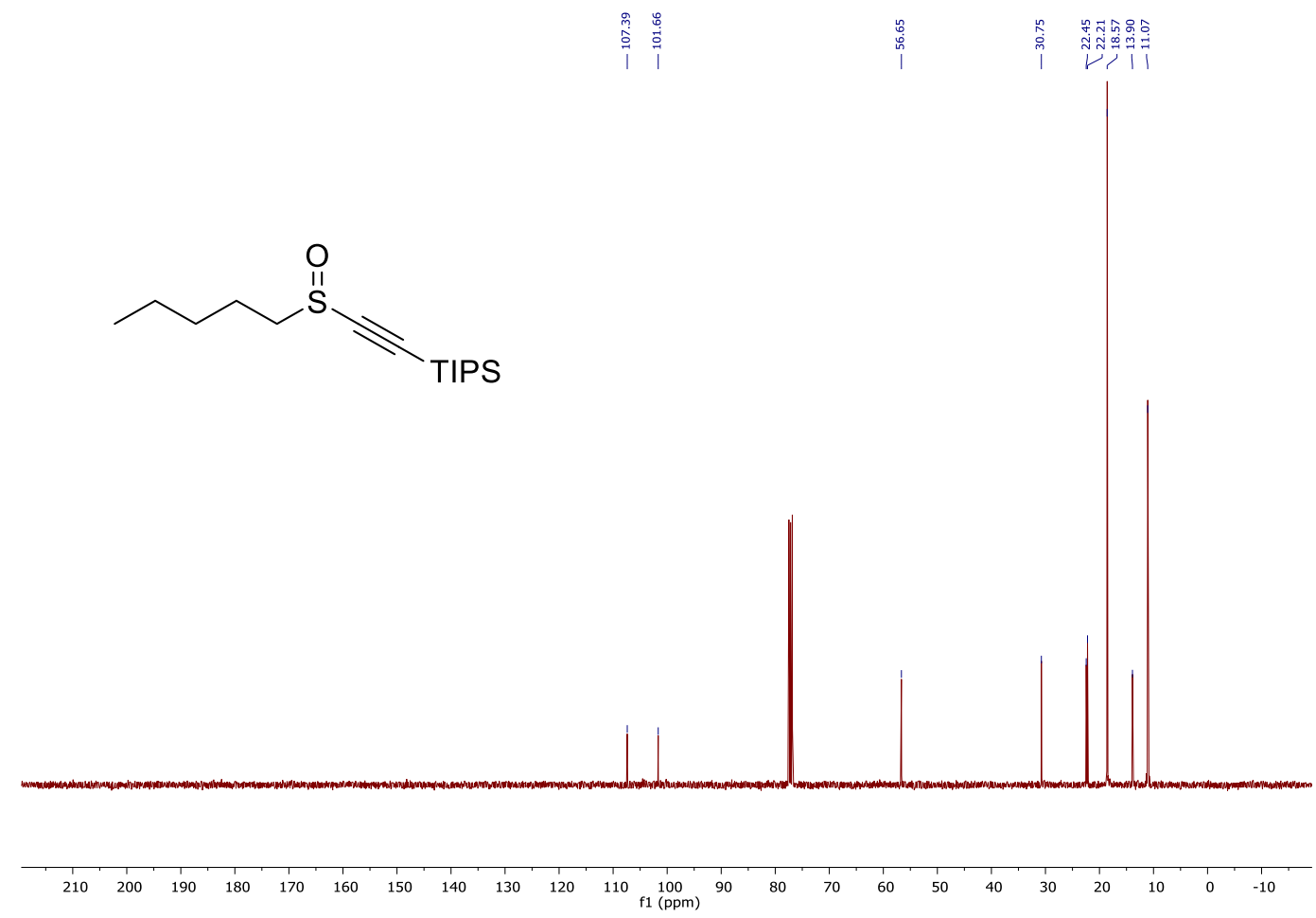




\section{Triisopropyl((cyclohexylsulfinyl)ethynyl)silane (10o)}

${ }^{1} \mathrm{H}$ NMR (400 MHz, Chloroform- $d$ )

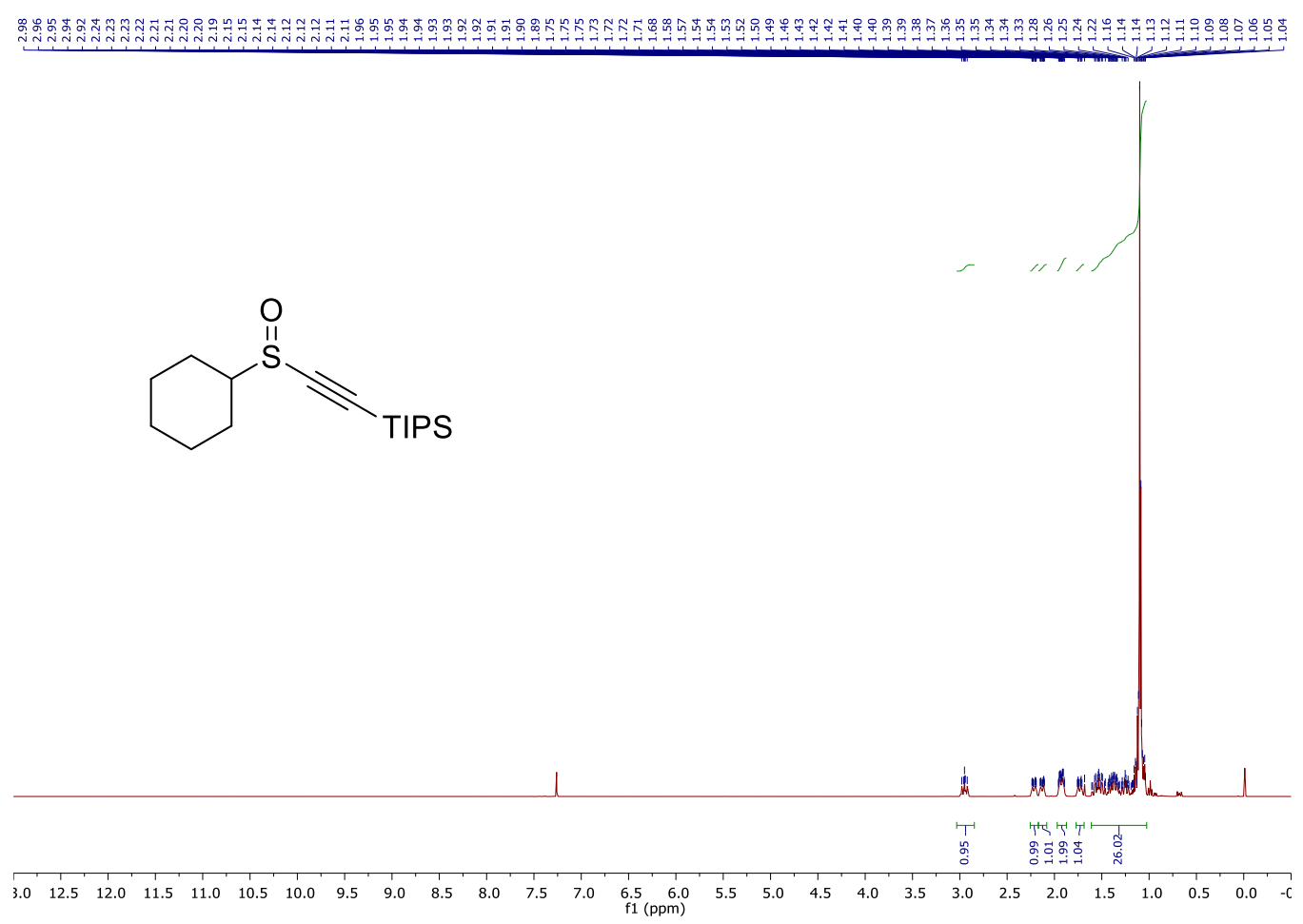

${ }^{13} \mathrm{C}$ NMR (101 MHz, Chloroform- $d$ )

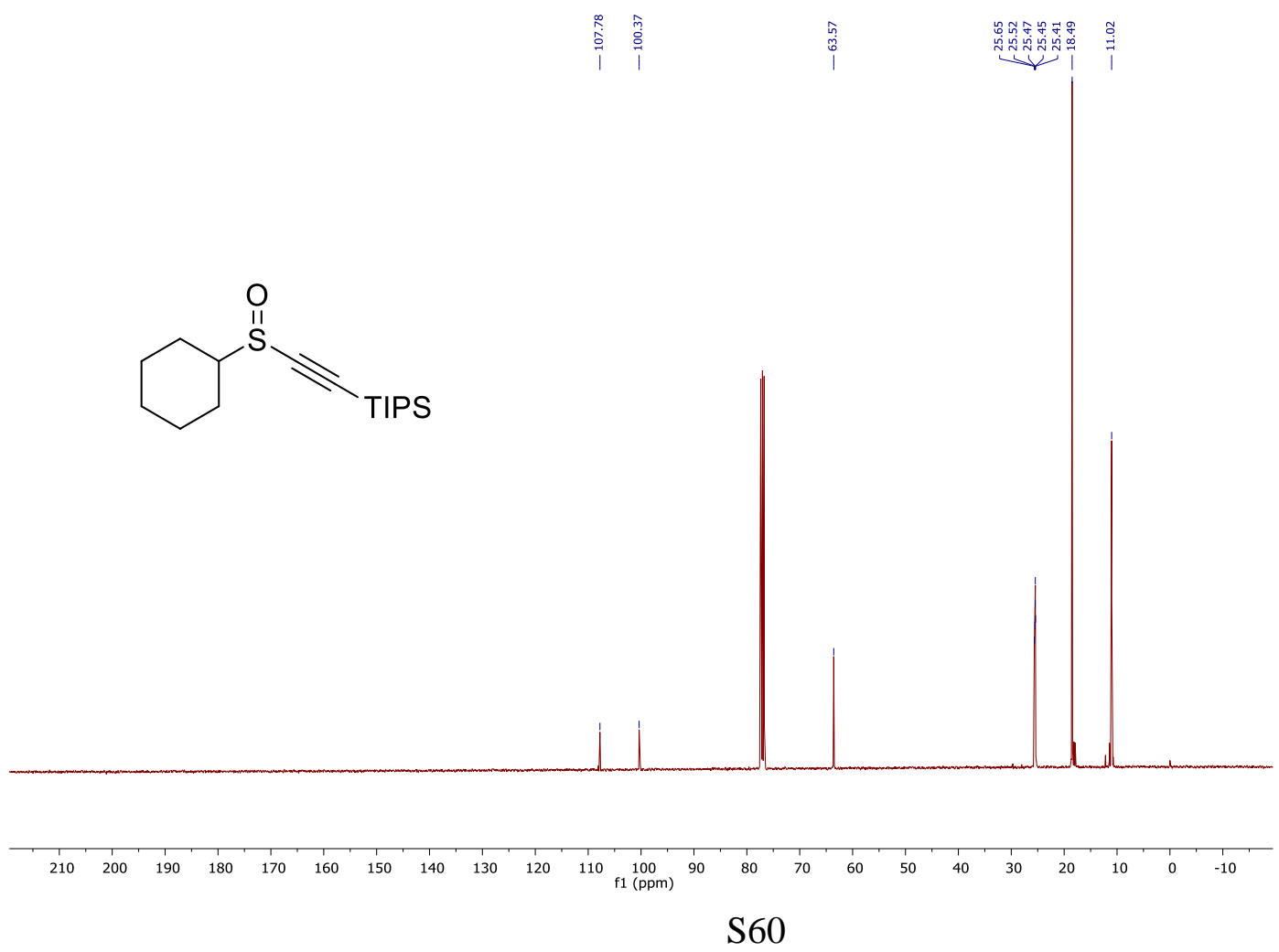




\section{Tert-butyldiphenyl((4-methylphenylsulfinyl)ethynyl)silane (10p)}

${ }^{1} \mathrm{H}$ NMR (400 MHz, Chloroform- $d$ )

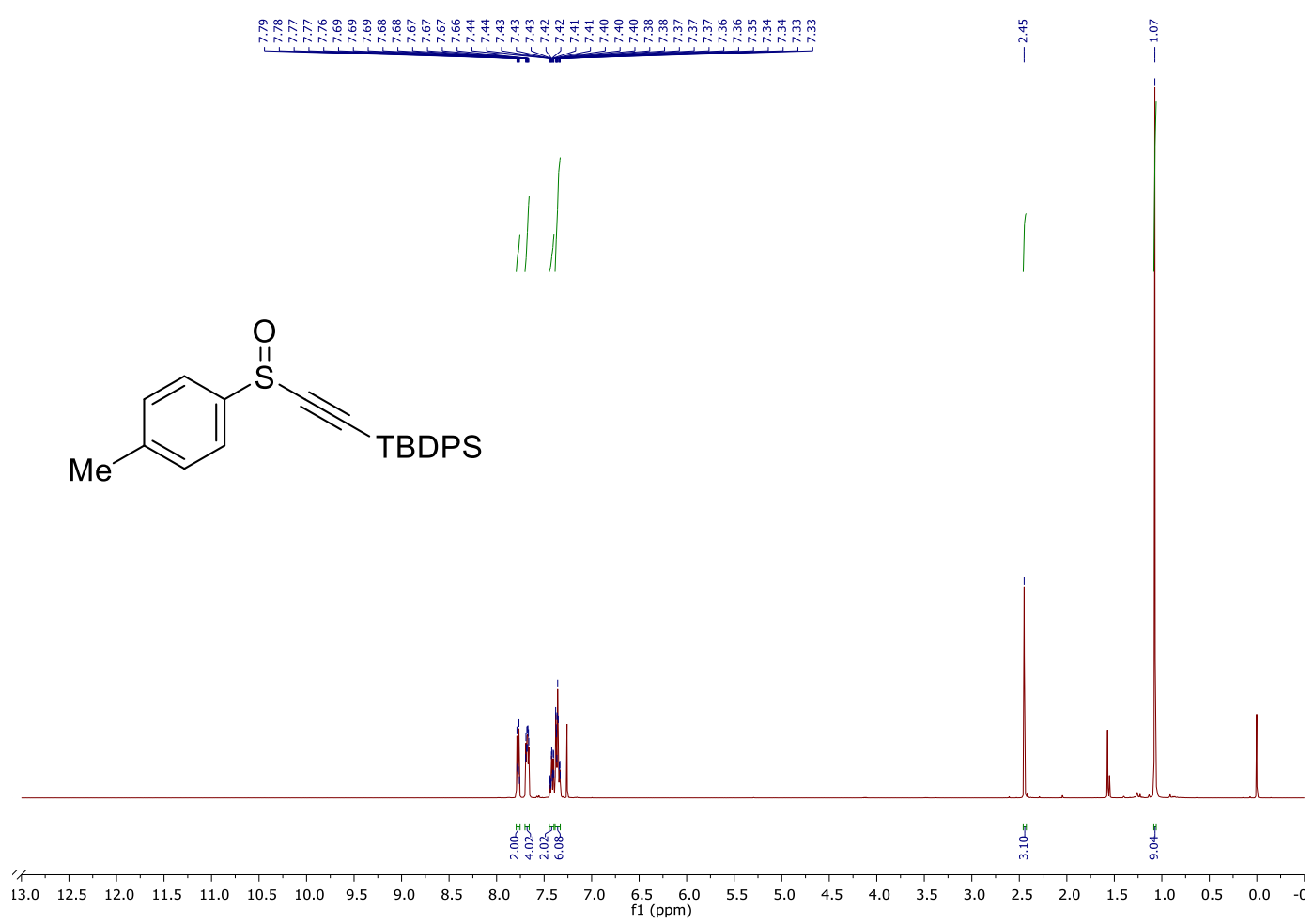

${ }^{13} \mathrm{C}$ NMR (101 MHz, Chloroform-d)

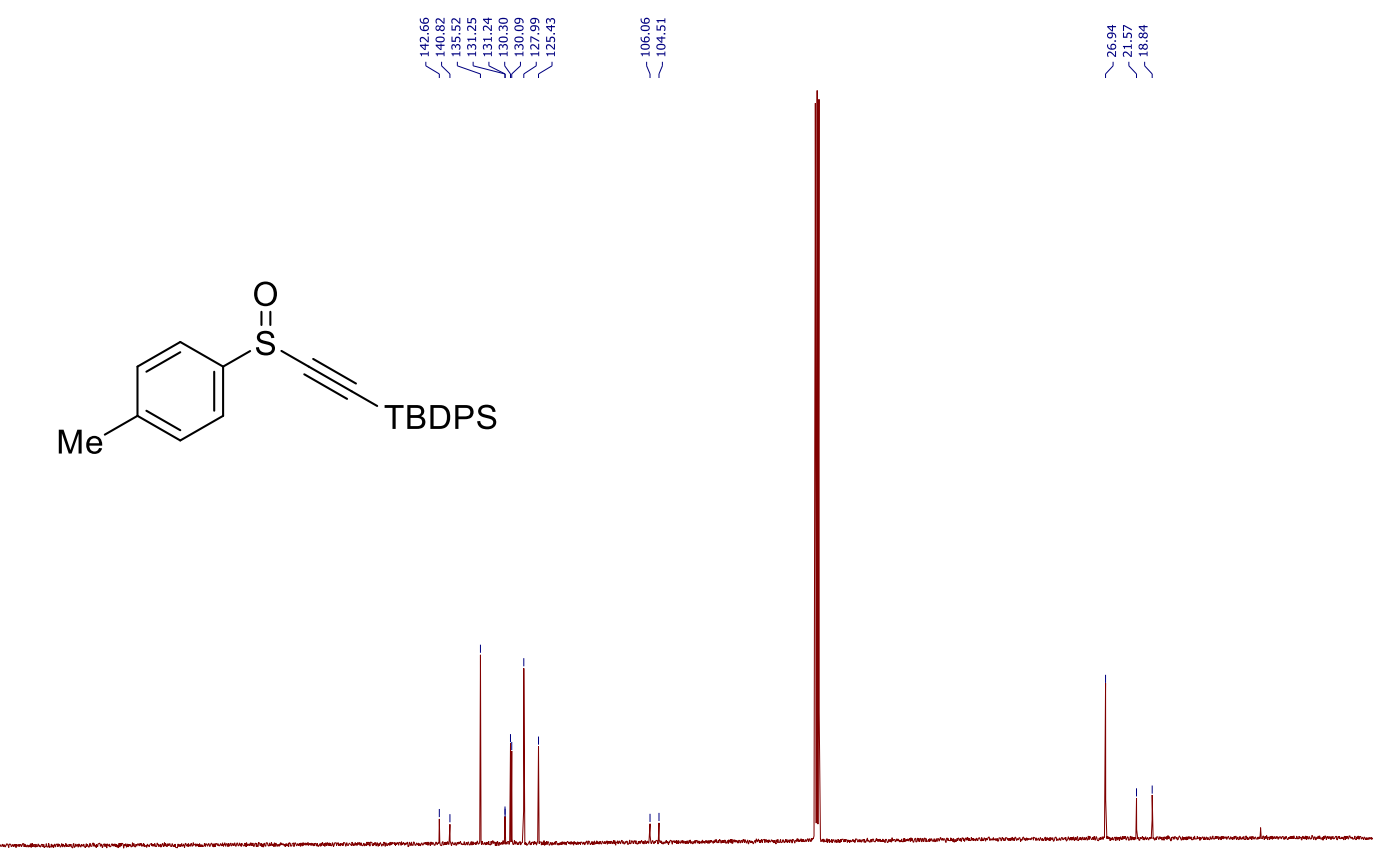

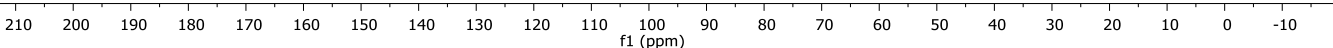




\section{Tert-butyl((4-methylphenylsulfinyl)ethynyl)silane (XX)}

${ }^{1} \mathrm{H}$ NMR (400 MHz, Methanol-d4)

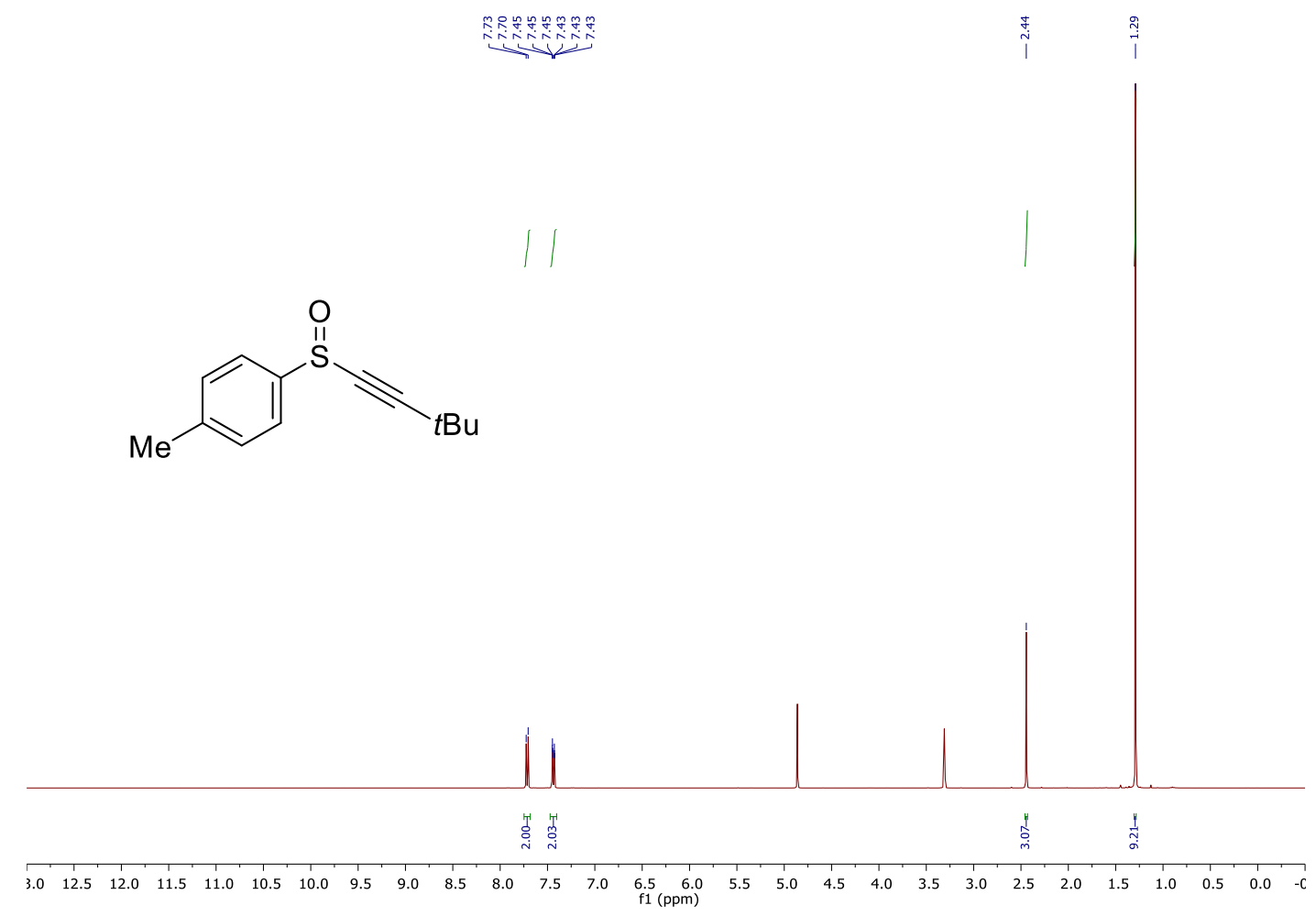

${ }^{13} \mathrm{C}$ NMR (101 MHz, Methanol- $\left.d_{4}\right)$

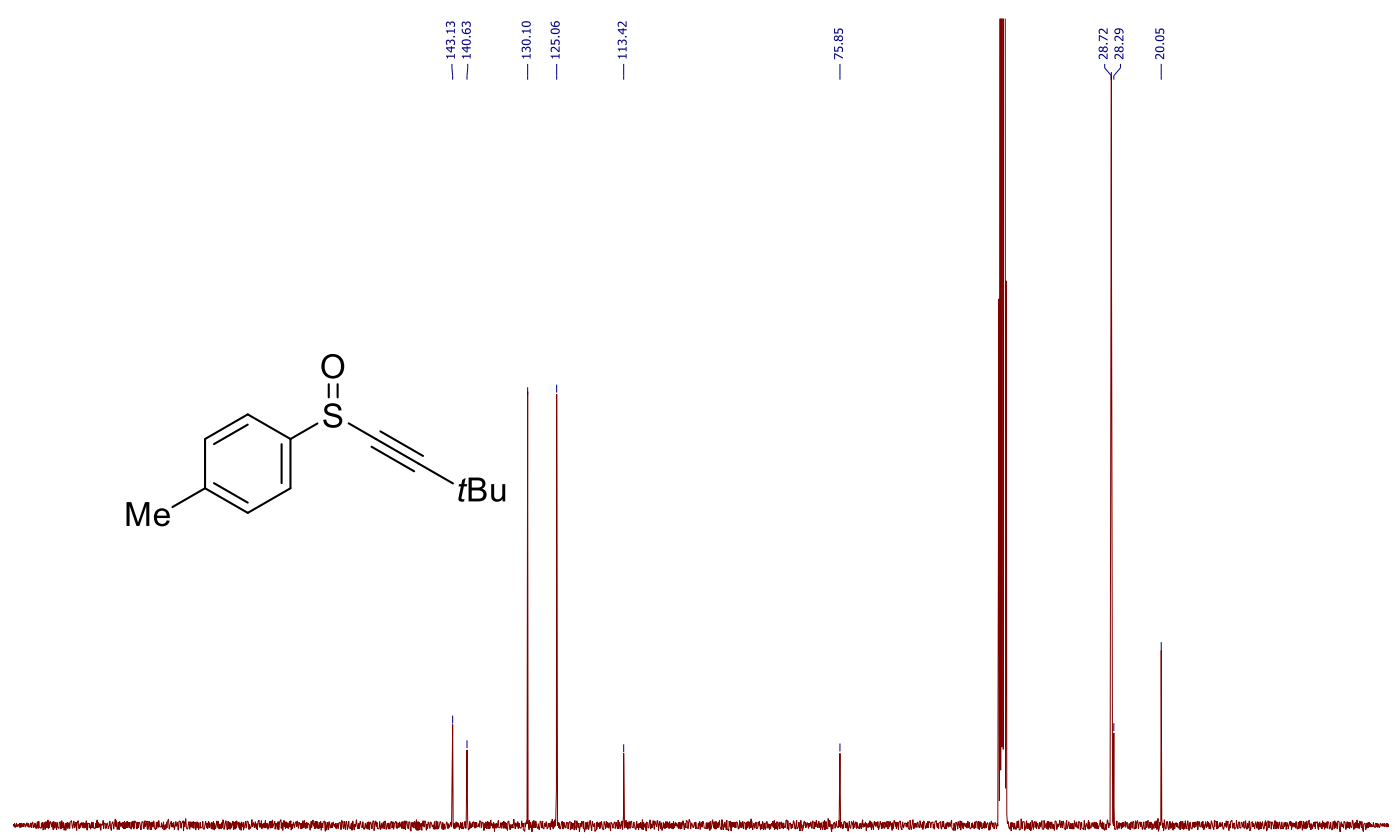

$\begin{array}{lllllllllllllllllllllllllllll}210 & 200 & 190 & 180 & 170 & 160 & 150 & 140 & 130 & 120 & 110 & 100 & 90 & 80 & 70 & 60 & 50 & 40 & 30 & 20 & 10 & 0 & -10\end{array}$ 


\section{Phenyl((4-methylphenylsulfinyl)ethenyl)silane (12a)}

${ }^{1} \mathrm{H}$ NMR (400 MHz, Chloroform- $d$ )

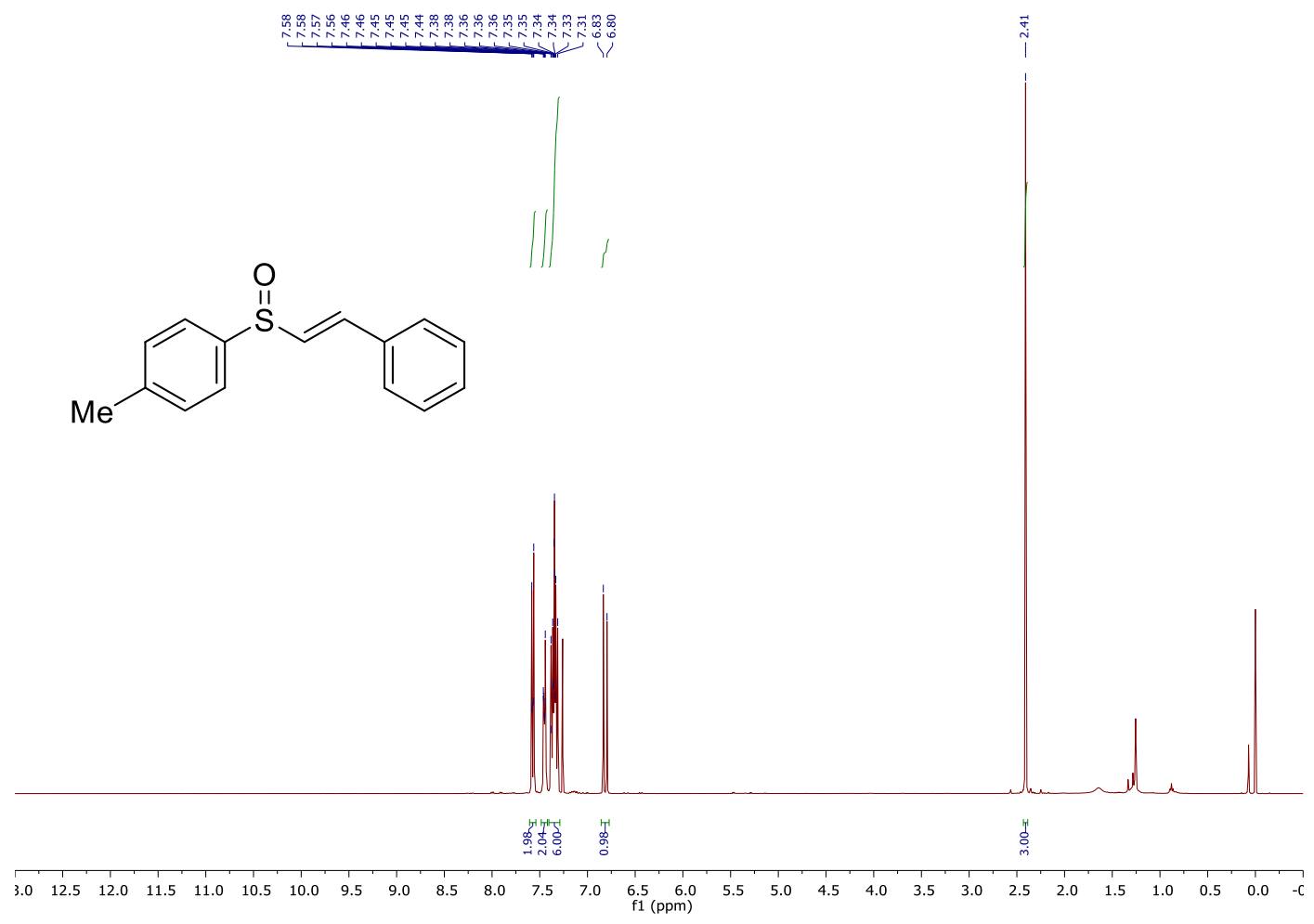

${ }^{13} \mathrm{C}$ NMR (101 MHz, Chloroform- $d$ )

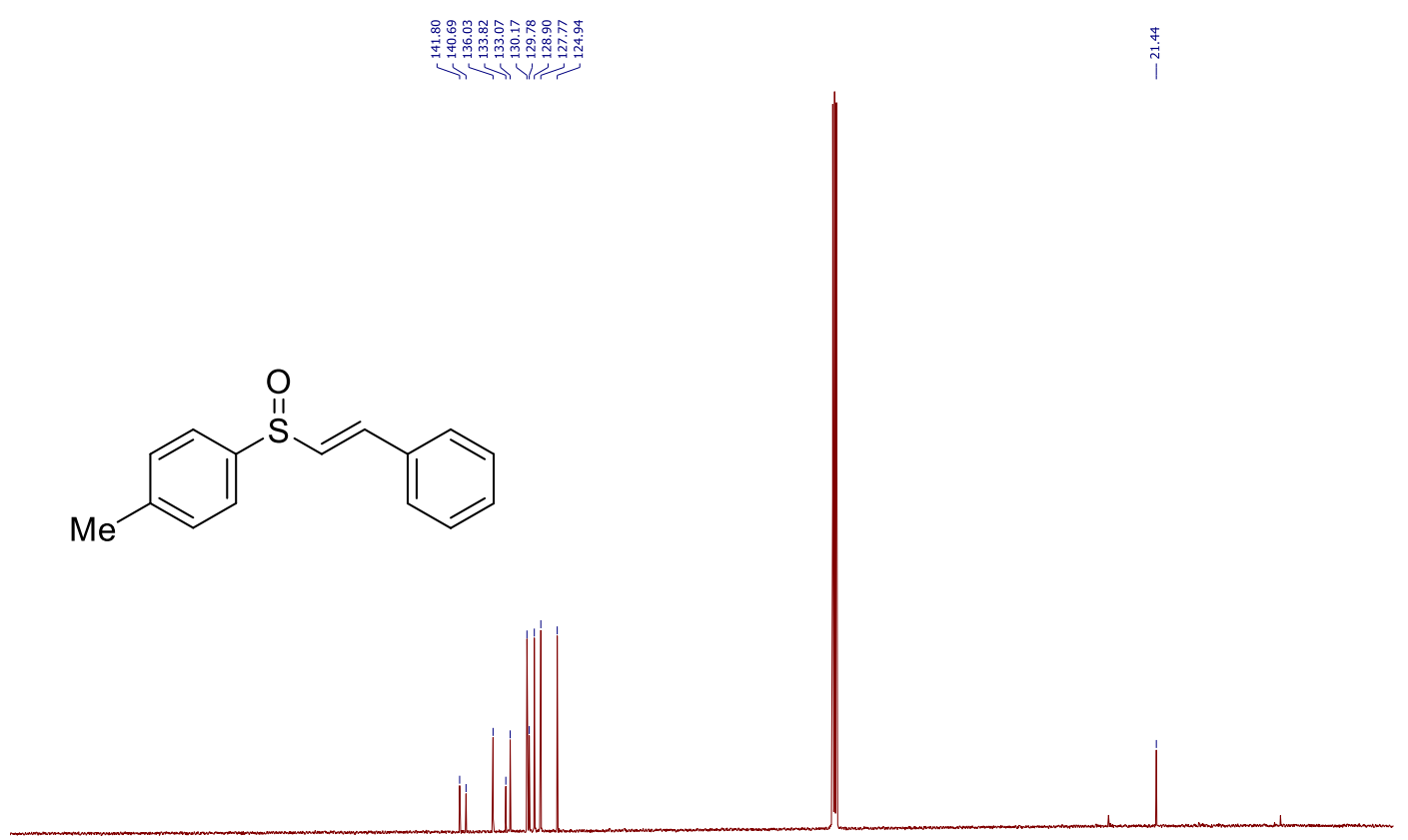

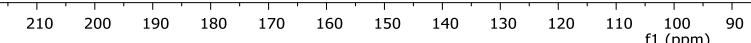




\section{4-methylphenyl((4-methylphenylsulfinyl)ethenyl)silane (12b)}

${ }^{1} \mathrm{H}$ NMR (400 MHz, Chloroform- $d$ )

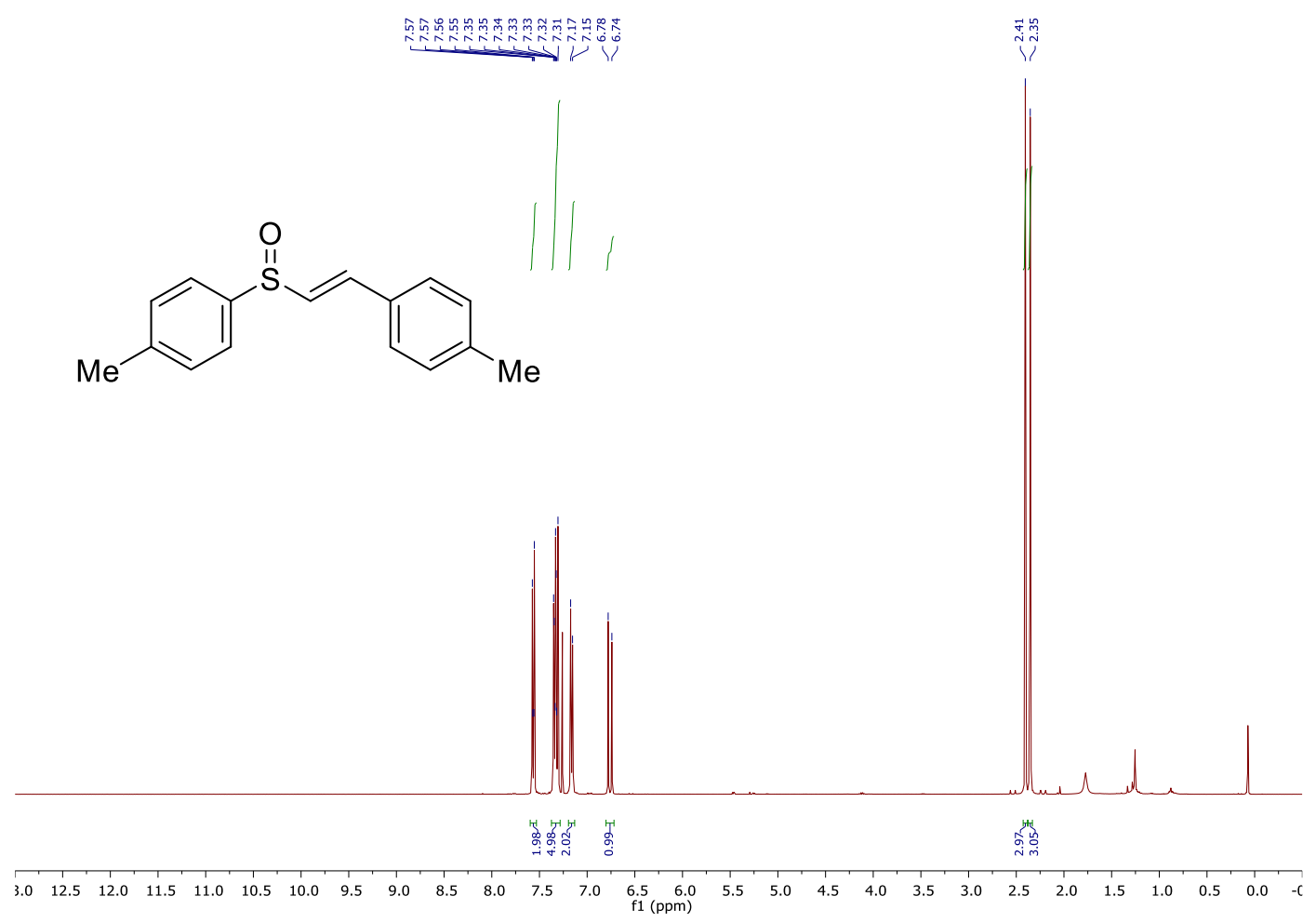

${ }^{13} \mathrm{C}$ NMR (101 MHz, Chloroform- $d$ )
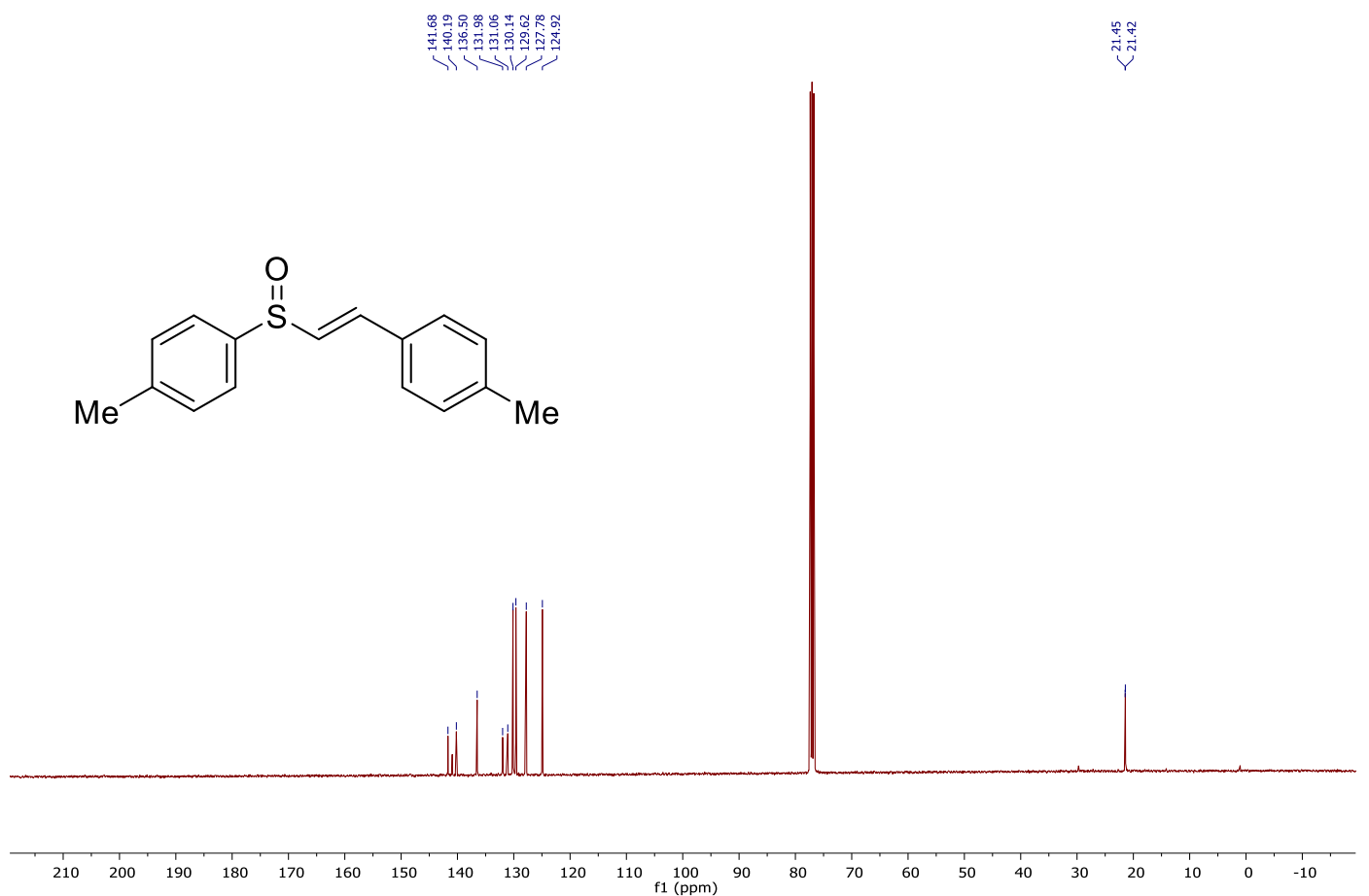


\section{1-(ethynylsulfinyl)-4-methylbenzene (13)}

${ }^{1} \mathrm{H}$ NMR (400 MHz, Chloroform- $d$ )

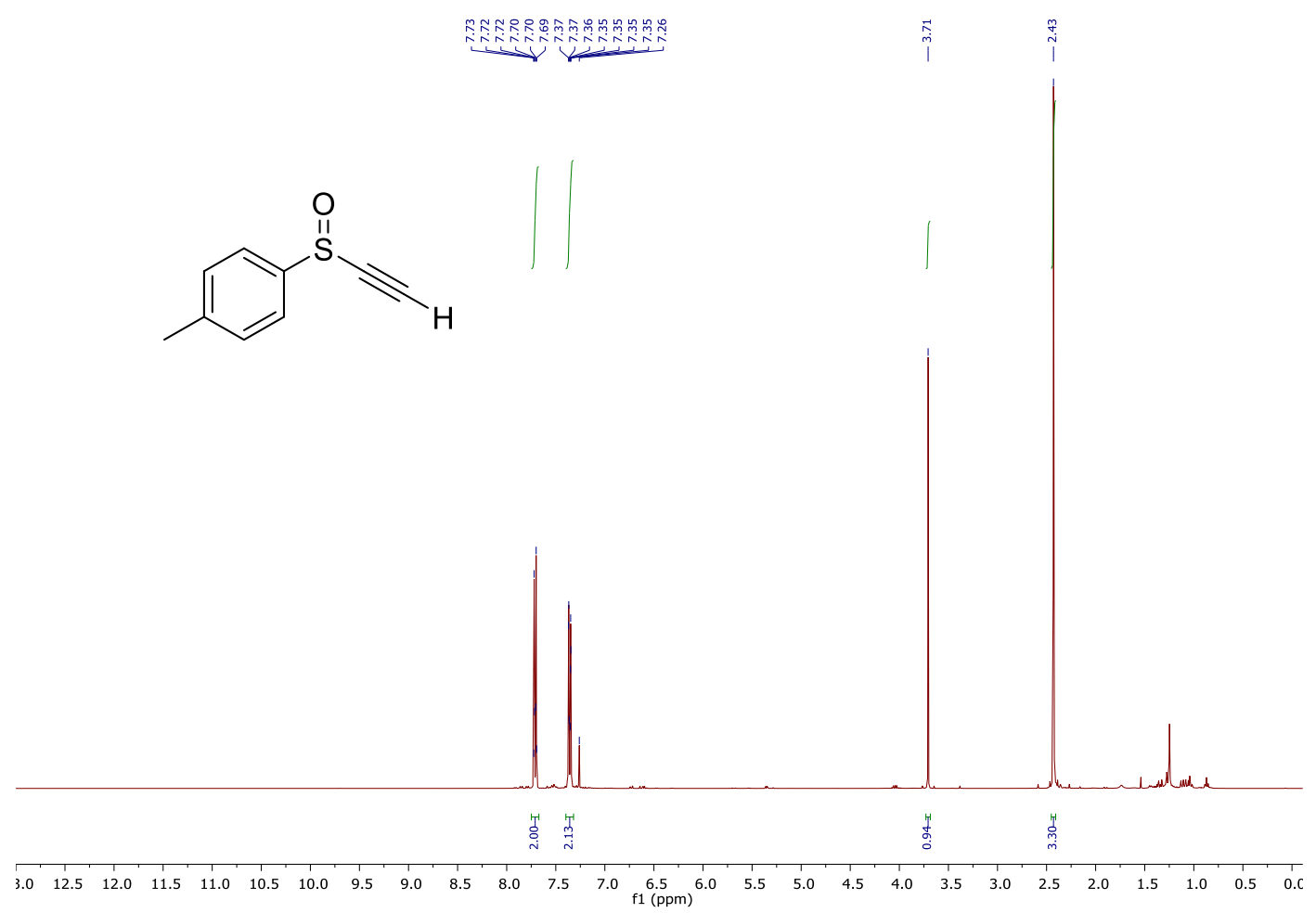

${ }^{13} \mathrm{C}$ NMR (101 MHz, Chloroform- $d$ )
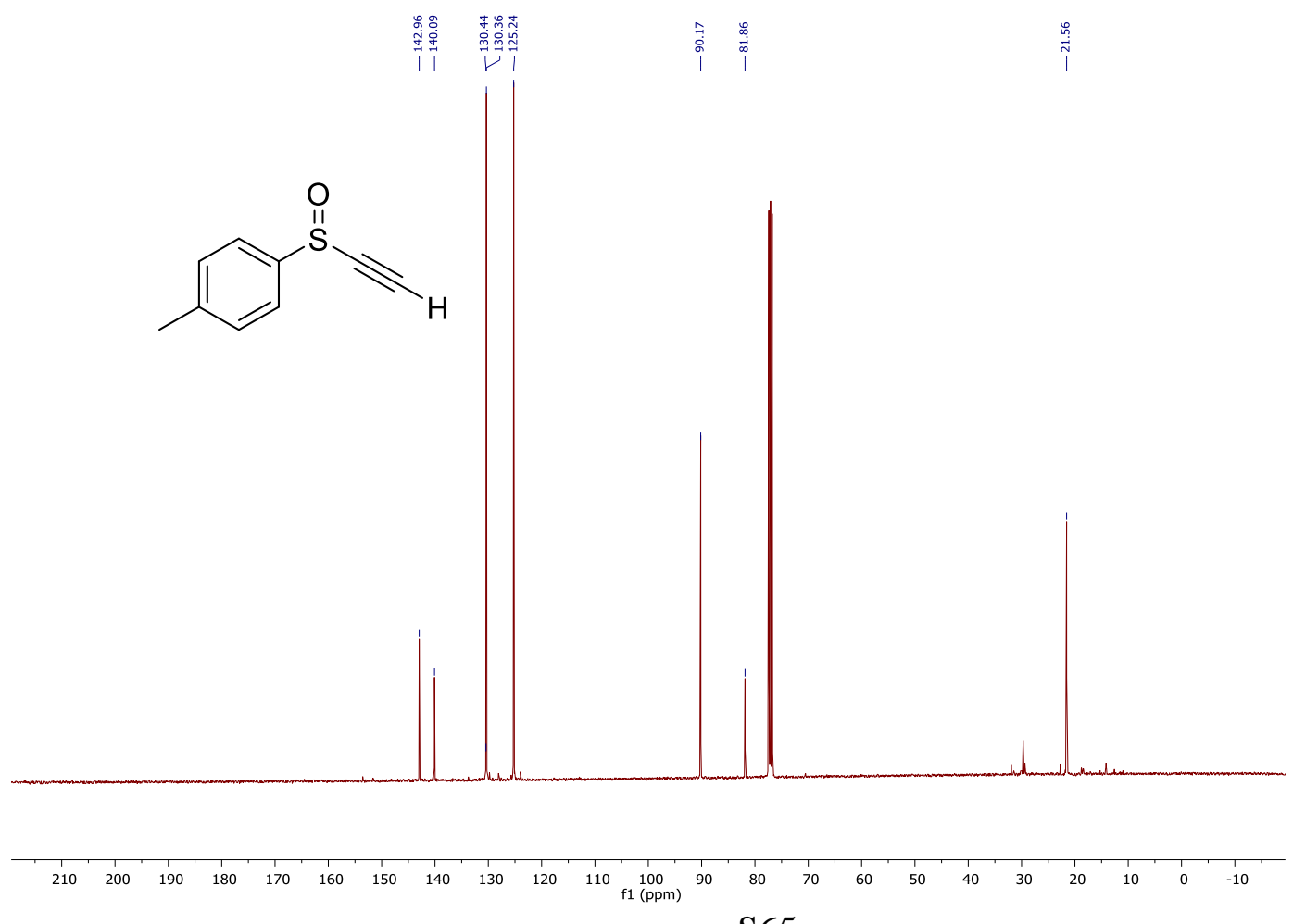